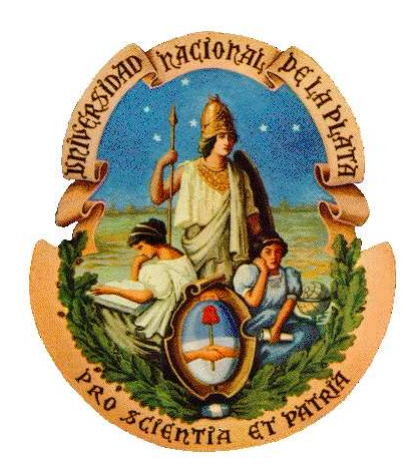

\title{
MODELADO Y ANÁLISIS DEL FLUJO DE AGUA EN MEDIOS POROSOS HETEROGÉNEOS TOTAL O PARCIALMENTE SATURADOS
}

\author{
por \\ Leonardo Bruno Monachesi \\ Geofísico, Universidad Nacional de La Plata
}

Director:

Dr. Luis Guarracino

TESIS DE DOCTORADO EN GEOFÍSICA

Facultad de Ciencias Astronómicas y Geofísicas

Universidad Nacional de la Plata

Febrero de 2013 



\title{
MODELADO Y ANÁLISIS DEL FLUJO DE AGUA EN MEDIOS POROSOS HETEROGÉNEOS TOTAL O PARCIALMENTE SATURADOS
}

\author{
Tesis PResentada en CUMPlimiento De los Requisitos \\ PARA OBTENER EL GRADO DE \\ Doctor en GeofísicA \\ en la \\ Facultad de Ciencias Astronómicas y Geofísicas \\ Universidad Nacional de La Plata
}

Por: Leonardo Bruno Monachesi

Director: Dr. Luis Guarracino

Jurado: Dra. Ana María Platzeck

Dr. J. Germán Rubino

Dr. Luis Vives

Facultad de Ciencias Astronómicas y Geofísicas

Universidad Nacional de La Plata

Paseo del Bosque s/n

(1900) La Plata

Argentina

Febrero de 2013

(C) Leonardo Bruno Monachesi, 2013 

A mi familia 


\section{Resumen}

El presente trabajo de Tesis aborda el problema de la caracterización del flujo de agua en medios porosos heterogéneos mediante el uso de métodos numéricos y soluciones analíticas. En primer lugar, se propone un experimento numérico que simula las técnicas de laboratorio usualmente empleadas para la obtención experimental de las relaciones constitutivas. Con este fin se resuelve la ecuación de flujo no saturado en estado estacionario en dominios bidimensionales y tridimensionales considerando campos de conductividad hidráulica altamente heterogéneos. La linealización del problema se realiza mediante un esquema de Picard y la aproximación numérica mediante métodos híbridos mixtos de elementos finitos. Este procedimiento numérico es utilizado para obtener parámetros hidráulicos efectivos y relaciones constitutivas de rocas fracturadas y rocas heterogéneas con distinto grado de correlación espacial. Se presenta además un análisis comparativo de las diferencias que surgen al representar las heterogeneidades en dos y tres dimensiones. La caracterización hidráulica de rocas fracturadas también es abordada mediante modelos analíticos. Utilizando conceptos de geometría fractal para representar la red de fracturas se derivan dos modelos constitutivos con expresiones analíticas cerradas para una y dos fases (agua y aire). Las expresiones analíticas son analizadas en términos de los parámetros involucrados, y validadas mediante su ajuste con datos experimentales y valores obtenidos con el experimento numérico descrito anteriormente. Por último, se analiza el efecto de la heterogeneidad de la conductividad hidráulica a escala de campo en acuíferos costeros afectados por la marea. Para ello se derivan soluciones analíticas exactas y aproximadas que permiten estimar las fluctuaciones inducidas en pozos para el caso de heterogeneidades lineales de la conductividad hidráulica. Las nuevas soluciones analíticas son comparadas con la correspondiente a un acuífero homogéneo y utilizadas para estimar parámetros hidráulicos a partir de datos experimentales disponibles en la literatura. 


\section{Índice}

Resumen vi vi

Listado de tablas $\quad x$

Listado de figuras $\quad$ xi

1 Introducción 1

1.1 Generalidades . . . . . . . . . . . . . . . . . . 1

1.2 Descripción de los contenidos . . . . . . . . . . . . . 5

2 Relaciones constitutivas para flujo no saturado $\quad 7$

2.1 Aproximación del continuo . . . . . . . . . . . . . . . . . 7

2.1.1 Descripción molecular y descripción continua de un fluido . . . . . 7

2.1.2 Porosidad y Volumen Representativo Elemental . . . . . . . . . . 10

2.2 Variables macroscópicas . . . . . . . . . . . . . . . . . . . . . 12

2.3 Ley de flujo de Buckingham-Darcy . . . . . . . . . . . . . . . . . . . 18

2.3.1 Flujo bifásico . . . . . . . . . . . . . . . . . . . . 19

2.4 Ecuación de Richards . . . . . . . . . . . . . . . . . . . . . . . . 20

2.5 Determinación experimental de las relaciones constitutivas . . . . . . . . 20

2.5.1 Determinación de la curva de contenido de agua . . . . . . . . 21

2.5.2 Determinación de la curva de conductividad hidráulica . . . . . . 22

2.6 Fórmulas predictivas y modelos constitutivos . . . . . . . . . . . . . . . . 24

2.6.1 Fórmula de Burdine . . . . . . . . . . . . . 25

2.6.2 Fórmula de Mualem . . . . . . . . . . . . . . . . . . . 28

2.7 Relaciones constitutivas para medios heterogéneos . . . . . . . . . . . . 30

3 Diseño de experimentos numéricos 2D 33

3.1 Introducción . . . . . . . . . . . . . . . . . . . . . . 33

3.2 Planteo matemático del experimento . . . . . . . . . . . . . . . 34

3.3 Formulación mixta débil . . . . . . . . . . . . . . . . . 36 
3.4 Método mixto de elementos finitos . . . . . . . . . . . . . . . . 37

3.5 Implementación numérica $\ldots \ldots \ldots . \ldots \ldots$

3.6 Esquema general de la simulación . . . . . . . . . . . . . . . . . . 42

3.7 Cálculo de las relaciones constitutivas . . . . . . . . . . . . . . . 43

3.8 Relaciones constitutivas para rocas fracturadas . . . . . . . . . . . . 46

3.9 Permeabilidad efectiva en rocas con heterogeneidades fractales . . . . . . 49

3.10 Conclusiones . . . . . . . . . . . . . . . . . . . . . . 53

4 Diseño de experimentos numéricos 3D $\quad 55$

4.1 Planteo matemático del experimento . . . . . . . . . . . . . 55

4.2 Cálculo de las relaciones constitutivas . . . . . . . . . . . . 56

4.3 Relaciones constitutivas para rocas fracturadas 3D . . . . . . . . 58

4.4 Conductividad hidráulica en medios porosos heterogéneos 2D y 3D . . . 61

4.4.1 Generación de la muestra de roca heterogénea . . . . . . . . . 62

4.4.2 Cálculo de la conductividad hidráulica efectiva . . . . . . . . 65

4.5 Conclusiones . . . . . . . . . . . . . . . . . . . . . 68

5 Modelos constitutivos analíticos para rocas fracturadas $\quad 69$

5.1 Introducción . . . . . . . . . . . . . . . . . . . . . . . . . . . 69

5.2 Descripción de la red de fracturas . . . . . . . . . . . . . . . 71

5.3 Modelo constitutivo bifásico . . . . . . . . . . . . . . . . . . . 74

5.3 .1 Criterio de ocupación de las fases . . . . . . . . . . . 74

5.3 .2 Saturaciones efectivas . . . . . . . . . . . . . 76

5.3 .3 Permeabilidades . . . . . . . . . . . . . . . . 76

5.3.4 Ajustes con datos experimentales . . . . . . . . . . . 81

5.4 Modelo constitutivo para el flujo no saturado de agua . . . . . . . . . . 83

5.5 Validación del modelo . . . . . . . . . . . . . . . . 85

5.6 Conclusiones . . . . . . . . . . . . . . . . . . . . 90

6 Análisis de acuíferos heterogéneos por el método de marea inducida 91

6.1 Introducción . . . . . . . . . . . . . . . . . . . . . . . . . . . 91

6.2 Ecuación de flujo horizontal para un acuífero confinado . . . . . . . . . 93

6.2 .1 Almacenamiento . . . . . . . . . . . . . . . . 94

6.2 .2 Ecuación de balance de masa $\ldots \ldots \ldots \ldots$ 
6.3 Modelo matemático y soluciones analíticas . . . . . . . . . . . . . . 97

6.3.1 Solución analítica para un acuífero homogéneo . . . . . . . . 98

6.3.2 Solución analítica exacta para un acuífero heterogéneo . . . . . . 101

6.4 Análisis de la solución analítica exacta . . . . . . . . . . . . . . . 103

6.5 Aproximación asintótica de la solución exacta . . . . . . . . . . . . 108

6.6 Estimación de la conductividad hidráulica mediante el método de marea inducida . . . . . . . . . . . . . . . . . . 110

6.6 .1 Resultados . . . . . . . . . . . . . . . . . . . 111

6.7 Conclusiones . . . . . . . . . . . . . . . . . . . . . . . . . . . 113

$\begin{array}{llr}7 \text { Conclusiones } & 115\end{array}$

$\begin{array}{ll}\text { Agradecimientos } & 119\end{array}$

$\begin{array}{ll}\text { Apéndice } & 120\end{array}$

A Resolución numérica del experimento 3D 120

A.1 Planteo matemático del experimento . . . . . . . . . . . . . 120

A.2 Formulación mixta débil . . . . . . . . . . . . . . . . . . . 121

A.3 Método mixto de elementos finitos . . . . . . . . . . . . . . . . 122

A.4 Implementación numérica . . . . . . . . . . . . . . . . . . . . 124

A.5 Esquema general de la simulación . . . . . . . . . . . . . . . 128

$\begin{array}{ll}\text { Referencias } & 130\end{array}$ 


\section{Listado de tablas}

3.1 Parámetros de van Genuchten para las fracturas y la matriz. . . . . . . . 48

5.1 Parámetros obtenidos mediante el ajuste de las curvas de permeabilidad relativa a los valores simulados por Liu y Bodvarsson y los datos medidos en Grimsel Test Site. . . . . . . . . . . . . . . . . . . . . . . .

5.2 Parámetros ajustados del modelo propuesto a los valores simulados para las muestras bidimensionales (a) y (b) generadas en el Capítulo 3 y la muestra tridimensional generada en el Capítulo 4. . . . . . . . . . . 88

6.1 Parámetros ajustados mediante los métodos de amplitud y de fase. . . . 112 


\section{Listado de figuras}

2.1 Curva teórica de densidad en función del volumen para un medio fluido (adaptada de [7]) . . . . . . . . . . . . . . . . .

2.2 Curva teórica de porosidad en función del volumen para un medio poroso (adaptada de [7]) . . . . . . . . . . . . . . . . . . . . . 12

2.3 Curvatura de la interfase aire-agua. . . . . . . . . . . . . . . . 17

2.4 Principio de medición en laboratorio del contenido de agua en función de la altura de presión. . . . . . . . . . . . . . . 21

2.5 Diagrama del dispositivo de medición de $K(h)$ mediante el método de flujo estacionario (adaptada de [7]) . . . . . . . . . . . . . 23

2.6 Modelo de tubos capilares rectos (a) y geometría poral de los modelos de Burdine (b) y Mualem (c) . . . . . . . . . . . . . . . . . 25

3.1 Representación matemática del dominio de simulación. . . . . . . . . . 35

3.2 Elemento $\Omega_{j, k}$ de la partición de elementos finitos. En (a) se ilustra la nomenclatura utilizada para la frontera y en (b) se muestran las incógnitas del problema y los puntos a los que están asociadas. . . . . . . . . 38

3.3 Muestras sintéticas de roca fracturada. . . . . . . . . . . . . . 47

3.4 Porosidad en función del tamaño de la muestra. . . . . . . . . . . . 48

3.5 Curvas de $S_{e}^{e f}\left(h^{e f}\right)$ (izquierda) y $K_{r}^{e f}\left(S_{e}^{e f}\right)$ (derecha). Resultado de la simulación para las muestras (a) y (b) y curvas de van Genuchten para las texturas de la matriz y las fracturas. . . . . . . . . . . . . . .

3.6 Curvas de $S_{e}^{e f}\left(h^{e f}\right)$ (izquierda) y $K_{r}^{e f}\left(S_{e}^{e f}\right)$ (derecha). Resultado de la simulación considerando un medio homogéneo con la textura de las fracturas y con la textura de la matriz. Los puntos corresponden a los valores simulados y las líneas de trazo a los modelos de van Genuchten (vG) para ambas texturas. . . . . . . . . . . . . . . . 50

3.7 Muestra de roca con heterogeneidades de tipo fractal. . . . . . . . . . . . 51

3.8 Módulo del campo de flujo (normalizado) resultante del experimento numérico. . . . . . . . . . . . . . . . . . . . . 53 
3.9 Curva del valor inverso del factor de calidad $1 / Q_{p}$ (arriba) y velocidad de fase $V_{p}$ (abajo) como función de la frecuencia para la muestra heterogénea de la Fig. 3.7 ( $\kappa$ het.) y para los campos de permeabilidad homogénea $\kappa^{e f}$, $\kappa_{A} \mathrm{y} \kappa_{H} \ldots \ldots \ldots \ldots \ldots$

4.1 Muestra 3D de medio poroso (izquierda) y representación matemática de su dominio (derecha). . . . . . . . . . . . . . . . .

4.2 Representación esquemática de la distribución espacial de las fracturas y sus dimensiones. . . . . . . . . . . . . . . . . . . . 58

4.3 Muestra de roca fracturada (a) y porosidad en función del tamaño de la muestra (b). . . . . . . . . . . . . . . . . .

4.4 Saturación efectiva simulada (izquierda) y conductividad hidráulica relativa simulada (derecha). . . . . . . . . . . . . . . . .

4.5 Muestra de roca fracturada (a) y gráficos del módulo de flujo normalizado para $h^{e f}=-30(\mathrm{~b}),-45$ (c) y -60 (d) cm. . . . . . . . . . . .

4.6 Realización 3D (izquierda) y gráfico de covarianza (derecha) para un campo $Y(\mathbf{x})$ generado utilizando el algoritmo turning bands. . . . . . . . . . . .

4.7 Conductividad hidráulica efectiva, expresada en $\mathrm{cm} / \mathrm{d}$, para la muestra 3D

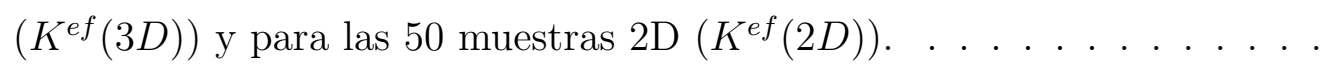

4.8 Conductividad hidráulica efectiva, expresada en cm/d, para los 50 cortes $2 \mathrm{D}$ de la muestra $\left(K^{e f}(2 D)\right)$ y sus respectivos valores equivalentes $K_{A}$, $K_{G}$ y $K_{H} \ldots \ldots \ldots \ldots$. . . . . . . . . . . . . . . 67

4.9 Módulo del vector de flujo 3D (izquierda) y 2D (derecha). Las tonalidades más claras corresponden a mayores intensidades. . . . . . . . . . . 67

5.1 Volumen representativo elemental (REV) de roca fracturada. . . . . . . . 72

5.2 Dos niveles de recursión de una carpeta de Sierpinski con dimensión fractal $D=1.84 \ldots \ldots \ldots \ldots \ldots \ldots$

5.3 Curvas de saturación efectiva de agua $S_{e, w}$ y de aire $S_{e, a}$ en función de la presión capilar efectiva $p_{c}^{e f}$. Para el ejemplo se ha considerado $p_{c, \text { mín }}=$

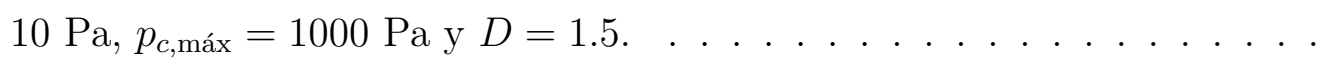

5.4 Curvas de permeabilidad relativa de agua para tres valores diferentes de dimensión fractal y $x_{\text {mín }} / x_{\text {máx }}=10^{-2}$ (izquierda) y para tres valores diferentes del rango de apertura de las fracturas y $D=1.5$ (derecha). . . . . 
5.5 Comparación entre los valores de $\kappa_{r, w}\left(S_{e}\right)$ simulados por Liu y Bodvarsson [59] y los valores obtenidos mediante el ajuste de la ecuación (5.21). .

5.6 Comparación entre los valores de $\kappa_{r, a}\left(S_{e}\right)$ medidos en Grimsel Test Site (GTS) y los valores obtenidos mediante el ajuste de la ecuación (5.22). .

5.7 Factor de tortuosidad $T$ como función de $S_{e}$ para los modelos de Burdine, de Liu y Bodvarsson y del modelo propuesto. . . . . . . . . . . . . . . . 86

5.8 Curvas de $S_{e}\left(h^{e f}\right)$ (izquierda) y $K_{r}\left(S_{e}\right)$ (derecha) ajustadas con los valores simulados para las muestras bidimensionales generadas en el Capítulo 3. Los gráficos superiores corresponden a la muestra (a) y los inferiores a la muestra (b). . . . . . . . . . . . . . . . .

5.9 Curvas de $S_{e}\left(h^{e f}\right)$ (izquierda) y $K_{r}\left(S_{e}\right)$ (derecha) ajustadas con los valores simulados para la muestra tridimensional generada en el Capítulo 4. . . . 88

6.1 Representación esquemática del flujo en un acuífero confinado bajo la aproximación de flujo horizontal (adaptada de [7]) . . . . . . . . . . .

6.2 Representación esquemática de un acuífero costero confinado. El eje $x$ está representado por la línea de puntos. . . . . . . . . . . . . .

6.3 Coeficiente de amplitud $c$ (izquierda) y fase temporal $\tau$ (derecha) versus distancia $x$ para un ejemplo hipotético, considerando dos valores de frecuencia angular de marea. . . . . . . . . . . . . . . . . . . . 100

6.4 Fluctuaciones de altura piezométrica inducidas por marea en función del tiempo en dos puntos de observación: (a) $a x=0.25$ y (b) $a x=2.0 . \quad$. . . 104

6.5 Coeficiente de amplitud $c$ versus distancia adimensional ax en escala logarítmica para diferentes tasas de incremento de la conductividad hidráulica. 105

6.6 Retraso temporal $\tau$ versus distancia adimensional ax para diferentes tasas de incremento de la conductividad hidráulica. . . . . . . . . . . . . . 106

6.7 Representación esquemática de un acuífero costero finito de longitud L. . 107

6.8 Coeficiente de amplitud $c$ versus distancia adimensional ax en escala logarítmica para dos acuíferos finitos y uno infinito. . . . . . . . . . . . 108

6.9 Coeficiente de amplitud $c$ (izquierda) y fase $\tau \omega$ (derecha) versus distancia adimensional ax para las soluciones exacta y aproximada. . . . . . . . . . 110 
6.10 Ajustes obtenidos para (a) $c(x)$ y (b) $\tau(x)$. La línea continua corresponde al modelo lineal, la línea de trazos al modelo homogéneo y los puntos a los datos de Schultz y Ruppel [93]. . . . . . . . . . . . . . . . . . . . 112

A.1 Dominio de simulación. . . . . . . . . . . . . . . . . . . . 120

A.2 Elemento $\Omega_{j, k, l}$ de la partición de elementos finitos. . . . . . . . . . . 123 


\section{Capítulo 1}

\section{Introducción}

\subsection{Generalidades}

A pesar de los avances logrados en los últimos años, la caracterización hidráulica precisa del subsuelo continúa siendo un desafío para investigadores de distintas disciplinas, como la hidrología, la geofísica, las ciencias del suelo y la ingeniería ambiental. La razón principal es la gran complejidad de los procesos involucrados y las heterogeneidades presentes a distintas escalas, las cuales demandan estudios con un alto grado de detalle a fin de garantizar una descripción hidráulica satisfactoria. Entre los numerosos problemas que requieren una caracterización hidráulica precisa se destacan la contaminación de acuíferos por causas naturales y antrópicas, el manejo de recursos limitados de agua en zonas áridas, la sobreexplotación de acuíferos costeros y la búsqueda de repositorios seguros tanto para el almacenamiento de residuos nucleares como para el secuestro de dióxido de carbono.

En términos generales la caracterización hidráulica del subsuelo consiste en la descripción cualitativa y cuantitativa del flujo de agua a través de un medio poroso. Una de las herramientas más potentes para avanzar en el conocimiento teórico y práctico de este proceso es la simulación computacional. Si los resultados obtenidos a partir de la simulación computacional se complementan con modelos teóricos analíticos, es posible realizar un estudio detallado de la dinámica del flujo y de los distintos factores que la controlan. No obstante ello, para garantizar la validez de los modelos y predicciones numéricas los mismos deben cotejarse con datos experimentales, los cuales no siempre están disponibles.

En la mayoría de los estudios del flujo de agua en medios porosos total o parcialmente saturados se utiliza la aproximación del continuo. Para el caso de saturación parcial, el flujo de agua se suele describir mediante la ecuación de Richards [84]. Esta ecuación es

altamente no lineal y para resolverla es necesario contar con relaciones constitutivas que vinculen la conductividad hidráulica, la saturación de agua y la altura de presión. 
La conductividad hidráulica es el parámetro que reviste mayor importancia pues controla la velocidad de transporte de los fluidos en el medio poroso. Además de ser función de las características litológicas, texturales y granulométricas del medio, la conductividad hidráulica depende fuertemente del grado de saturación de agua. Desafortunadamente, la determinación experimental de este parámetro es una tarea difícil y costosa. En el caso particular de medios porosos heterogéneos (como suelos con macroporos o rocas fracturadas) los datos experimentales son prácticamente inexistentes, por lo que suelen utilizarse modelos teóricos [103].

Una alternativa a la medición directa es la utilización de procedimientos numéricos que simulen las pruebas de laboratorio para determinar las relaciones constitutivas [25, $51,59]$. De esta manera, si se ha caracterizado adecuadamente al medio poroso (tanto desde el punto de vista geométrico como físico) la simulación computacional del flujo de agua nos permitirá obtener las relaciones constitutivas, como así también estudiar su dependencia con los parámetros físicos del medio. Esta metodología es particularmente útil al tratar con medios porosos altamente heterogéneos, donde la conductividad hidráulica suele registrar variaciones de gran magnitud.

Otra alternativa a la medición directa es el uso de modelos constitutivos analíticos. Estos modelos consisten en expresiones analíticas cerradas que permiten describir en forma continua las curvas de saturación y conductividad hidráulica en función de la altura de presión. Los modelos constitutivos más utilizados son los de van Genuchten [108], Brooks-Corey [13] y Gardner-Russo [35, 89].

La curva de conductividad hidráulica suele estimarse a partir de la curva de saturación, que en la mayoría de los casos puede obtenerse sin dificultad mediante ensayos de laboratorio [108]. Durante las últimas seis décadas se han presentado numerosas fórmulas predictivas que permiten obtener la curva de conductividad hidráulica a partir de la curva de saturación. A esta categoría pertenecen las fórmulas propuestas por Burdine [15] y Mualem [73], que han sido desarrolladas para el flujo no saturado de agua en rocas sedimentarias clásticas (medios porosos granulares). La aplicabilidad de estas fórmulas no puede en principio extenderse a medios porosos heterogéneos, dado que han sido concebidas bajo la hipótesis de homogeneidad de los medios que representan.

Las rocas fracturadas pueden considerarse como un tipo particular de medio poroso heterogéneo. Su estudio se ha convertido en un campo de activa investigación en los últimos 25 años, motivado principalmente por la búsqueda de sitios para repositorios de 
desechos nucleares y más recientemente para el secuestro de dióxido de carbono [10, 11, 5]. Por otra parte, en muchos sistemas acuíferos confinados, como el Guaraní, las rocas fracturadas participan en la recarga en forma indirecta $[85,37]$, por lo que resulta de interés modelar en forma adecuada el flujo de agua en este tipo de medio. Finalmente se menciona un tema de gran interés actual que es la fracturación hidráulica de reservorios no convencionales de hidrocarburos. Esta técnica consiste en la fracturación de la roca madre mediante la inyección de grandes volúmenes de agua con el fin de aumentar localmente la permeabilidad de la roca y permitir una mayor producción de hidrocarburos [114]. Esta técnica está siendo utilizada en nuestro país en la formación Vaca Muerta, la cual es considerada uno de los mayores reservorios no convencionales del planeta.

Cuando se utiliza la aproximación del continuo, la roca fracturada es considerada un medio continuo equivalente, caracterizado por un modelo constitutivo especialmente diseñado para este tipo de roca. Para describir una roca fracturada se suele suponer que la misma está constituida por una matriz homogénea de baja o nula permeabilidad, con una red de fracturas interconectadas. Las fracturas se conceptualizan como un medio poroso de alta permeabilidad, cuya distribución espacial y propiedades geométricas se suponen conocidas.

Los modelos constitutivos para rocas fracturadas son muy escasos, y algunos de ellos se basan en fórmulas predictivas clásicas. Entre ellos podemos mencionar los modelos de Liu y Bodvarsson [59], Guarracino [39] y Guarracino y Quintana [42], los cuales utilizan la fórmula predictiva de Burdine con diferentes expresiones analíticas para la curva de saturación.

A escala de campo las heterogeneidades espaciales de la conductividad hidráulica también suelen tener un efecto significativo sobre el patrón de flujo de las aguas subterráneas. Es por esta razón que se dedican enormes esfuerzos a la determinación de la conductividad hidráulica a esta escala, como así también al diseño de modelos teóricos que permitan explicar dichas heterogeneidades y predecir sus efectos.

Dentro de los numerosos problemas en los que los acuíferos están afectados por heterogeneidades a escala de campo, centraremos la atención en los acuíferos costeros. Dichos sistemas han sido objeto de estudios minuciosos por parte de los especialistas de diversas áreas. Las enormes inversiones que suelen destinarse al estudio y caracterización de los acuíferos costeros tienen como objetivo principal garantizar la sustentabilidad del recurso ante la intrusión marina. Una característica particular de los acuíferos costeros 
es que suelen estar afectados por el efecto de la marea. En los pozos costeros de aguas subterráneas se observa una marea inducida que en los últimos años ha conducido al desarrollo de un método específico que permite estimar los parámetros hidráulicos del acuífero. Este método, denominado método de marea inducida, constituye una alternativa a los clásicos ensayos de bombeo que no siempre son factibles de realizar cerca de la costa.

Desde los años 50 se ha presentado un gran número de expresiones analíticas cuyo objeto es describir la interacción, inducida por la marea, entre el mar y el agua subterránea. Jacob [44] y Ferris [30] fueron los primeros en obtener una expresión analítica para un acuífero confinado y homogéneo. Debido a su simplicidad, dicha expresión fue ampliamente utilizada para estimar la conductividad hidráulica en acuíferos costeros. Entre los numerosos trabajos que utilizan esta expresión se destacan los de Carr y van der Kamp [18], Drogue et al. [27], Erskine [29] y Millham y Howes [64].

Más recientemente se han propuesto soluciones analíticas más complejas con el propósito de considerar efectos que hasta entonces no se habían tenido en cuenta, tales como el goteo, el almacenamiento, el efecto de carga en acuíferos submarinos y la presencia de heterogeneidades de la conductividad hidráulica. Este último efecto ha sido considerado por muy pocos autores, a pesar de que la mayoría de los acuíferos costeros presentan heterogeneidad y anisotropía en sus propiedades hidráulicas [54, 101].

En líneas generales el objetivo principal del presente trabajo de Tesis consiste en el análisis del efecto que produce la presencia de heterogeneidades en la conductividad hidráulica sobre el flujo de agua en condiciones de saturación parcial y total. Con este propósito se desarrollan e implementan experimentos numéricos en dominios bidimiensionales y tridimensionales capaces de caracterizar medios heterogéneos y de simular condiciones similares a las que se suele someter a las muestras de roca en laboratorios especializados. En este análisis se presta particular atención a las rocas fracturadas, para las cuales se desarrollan modelos constitutivos teóricos para el flujo en condiciones de saturación parcial, los cuales son validados con datos experimentales y numéricos. Por último se analiza a escala de campo el efecto de las heterogeneidades de la conductividad hidráulica sobre el flujo inducido por la marea en acuíferos costeros. A tal fin se propone para la conductividad hidráulica un modelo de variación lineal con la distancia a la costa y se obtienen soluciones analíticas que permiten analizar y cuantificar su efecto. 


\subsection{Descripción de los contenidos}

Teniendo en cuenta los objetivos planteados y las tareas propuestas, la presentación de los contenidos del trabajo de Tesis ha sido estructurada en siete Capítulos.

En el Capítulo 2 se presentan los fundamentos teóricos del flujo en medios porosos bajo la aproximación del continuo. Se introduce el concepto de relación constitutiva y se realiza una breve reseña de los métodos para su determinación experimental. Luego se presentan las fórmulas predictivas y modelos constitutivos clásicos desarrollados para medios porosos homogéneos. Por último se plantea el problema de la obtención de relaciones constitutivas para medios porosos heterogéneos, centrando la atención al caso particular de las rocas fracturadas.

En el Capítulo 3 se presenta un método numérico para el cálculo de relaciones constitutivas en muestras sintéticas bidimensionales de rocas. El método se basa en la simulación de un experimento de laboratorio, para lo cual es necesario resolver la ecuación de Richards en estado estacionario con condiciones de borde apropiadas. Se propone un método mixto de elementos finitos para la resolución del problema en muestras heterogéneas generales. Finalmente, se presentan ejemplos ilustrativos de la aplicación del método a la caracterización hidráulica de rocas fracturadas y al análisis del comportamiento de la llamada permeabilidad sísmica.

La implementación tridimensional del método numérico para el cálculo de relaciones constitutivas se presenta en el Capítulo 4. El algoritmo para la resolución numérica del problema se detalla en el Apéndice A. La aplicación del método se ilustra con el cálculo de las relaciones constitutivas en una muestra tridimensional de roca fracturada. Por último se incluye un estudio comparativo de la conductividad hidráulica efectiva estimada mediante simulaciones en 2 y 3 dimensiones en rocas que presentan heterogeneidades de tipo lognormal en la conductividad hidráulica.

En el Capítulo 5 se presentan dos modelos constitutivos analíticos diseñados para describir el flujo bifásico (aire y agua) y el flujo monofásico (agua) en rocas fracturadas. Se propone un modelo conceptual de tipo fractal para la representación de la red de fracturas y se plantean las hipótesis y las leyes físicas que se asumen en la derivación de las expresiones analíticas. La validación de los modelos propuestos se realiza mediante la comparación con modelos existentes, datos experimentales y las relaciones constitutivas simuladas en los Capítulos 3 y 4. 
En el Capítulo 6 se presenta la derivación de dos soluciones analíticas (una exacta y otra aproximada) para modelar la respuesta hidráulica inducida por marea en acuíferos costeros heterogéneos. Se considera un modelo simplificado para la representación del acuífero y se asume una heterogeneidad de tipo lineal para la conductividad hidráulica. El efecto de la heterogeneidad se evalúa mediante el análisis de la respuesta espacial y temporal del acuífero ante las fluctuaciones inducidas por marea. Para finalizar, la solución analítica exacta es utilizada para estimar la conductividad hidráulica en un caso real a partir del método de marea inducida.

Finalmente, en el Capítulo 7 se presentan las conclusiones del trabajo de Tesis. 


\section{Capítulo 2}

\section{Relaciones constitutivas para flujo no saturado}

En este Capítulo se presenta una síntesis de los fundamentos teóricos a partir de los cuales se desarrolla gran parte de esta Tesis. Se introducen los conceptos de medio continuo y de volumen representativo elemental $\left(\mathrm{REV}^{1}\right)$ y se definen las variables macroscópicas y las leyes físicas que gobiernan el fenómeno del flujo en medios porosos no saturados. Esto nos lleva a la definición de las relaciones constitutivas, las cuales son indispensables para la caracterización completa de un medio poroso desde el punto de vista hidráulico. Se presentan algunos de los métodos experimentales de laboratorio para la estimación de las relaciones constitutivas, y se realiza una reseña de las fórmulas predictivas y de los modelos analíticos clásicos desarrollados para medios porosos homogéneos. Los contenidos del presente Capítulo son el resultado de una recopilación bibliográfica de textos clásicos y publicaciones actuales.

\subsection{Aproximación del continuo}

En esta sección se introduce el concepto de medio continuo (o aproximación del continuo) aplicado al estudio del flujo de agua en medios porosos total o parcialmente saturados. Esta aproximación prescinde de la descripción exacta de la geometría interna del medio poroso, lo que permite abordar problemas de interés hidrogeofísico.

\subsubsection{Descripción molecular y descripción continua de un fluido}

Un fluido está constituido por un gran número de moléculas, moviéndose unas respecto de otras, chocando entre sí e intercambiando energía y cantidad de movimiento. La física clásica cuenta con herramientas suficientes para describir en forma completa este tipo de sistemas. Bastaría con conocer las posiciones, momentos iniciales y las fuerzas actuantes sobre cada molécula para determinar su evolución futura. Sin embargo esta tarea no es simple, aún con la disponibilidad actual de computadoras de alta velocidad y gran

\footnotetext{
${ }^{1}$ del inglés Representative Elementary Volume.
} 
capacidad de cálculo.

Como alternativa a esta descripción molecular es posible adoptar una aproximación diferente, de naturaleza estadística, para obtener información acerca de un sistema compuesto por un gran número de moléculas. Para tal sistema, la mecánica estadística permite determinar sus propiedades físicas a partir de las leyes de movimiento de las partículas individuales. La aplicación de estas herramientas estadísticas nos permitiría pasar de una escala molecular de estudio a la escala continua, de la misma manera en que en la teoría cinética de los gases las variables mecánicas del movimiento de las moléculas de un gas, como por ejemplo cantidad de movimiento y energía cinética, se traducen a variables estadísticas como presión y temperatura. A esta aproximación estadística se la denomina aproximación del continuo [7]. Al igual que en la descripción molecular, la aproximación del continuo requiere del concepto de partícula. En este caso, una partícula de fluido está constituida por un ensamble de muchas moléculas contenidas en un volumen pequeño. Su tamaño debe ser mayor que el camino libre medio de una única molécula, pero lo suficientemente pequeño en comparación con el dominio fluido considerado, de modo que promediando las propiedades del fluido y del movimiento de las moléculas contenidas en dicho volumen, puedan obtenerse valores medios representativos. Estos valores quedan asociados a un único punto del dominio, coincidente con el centro del volumen considerado, que se denomina centroide [7]. Luego, todo punto del dominio ocupado por un fluido posee propiedades cinemáticas y dinámicas características.

Al introducir el concepto de partícula queda implícita la existencia de un volumen $V$ asociado a ella. La densidad del fluido que ocupa tal volumen es el cociente entre la masa $m$ del fluido y dicho volumen. Sin embargo, el valor de la densidad calculado de esta manera no será independiente del volumen de la partícula fluida.

Siguiendo a Prandtl y Teitjens [81], consideremos un punto $P$ del fluido, y sea $m_{i}$ la masa del fluido en un volumen representativo $V_{i}$ tal que $P$ sea su centroide. La densidad media $\rho_{i}$ del fluido es $\rho_{i}=m_{i} / V_{i}$. Supongamos que realizamos este cálculo para una secuencia de volúmenes progresivamente más chicos $V_{1}>V_{2}>V_{3} \ldots$ Los resultados de este cálculo teórico podrían representarse por la gráfica esquemática de la Fig. 2.1.

Si comenzamos el análisis con un $V_{1}$ suficientemente grande, se observarán cambios graduales en la densidad media, especialmente si el fluido es inhomogéneo. Las fluctuaciones en torno de $\rho_{i}$ disminuyen a medida que $V_{i}$ se hace más pequeño. Luego, a medida que $V_{i}$ converge sobre $P$ se observa un rango en el que no ocurren cambios de 


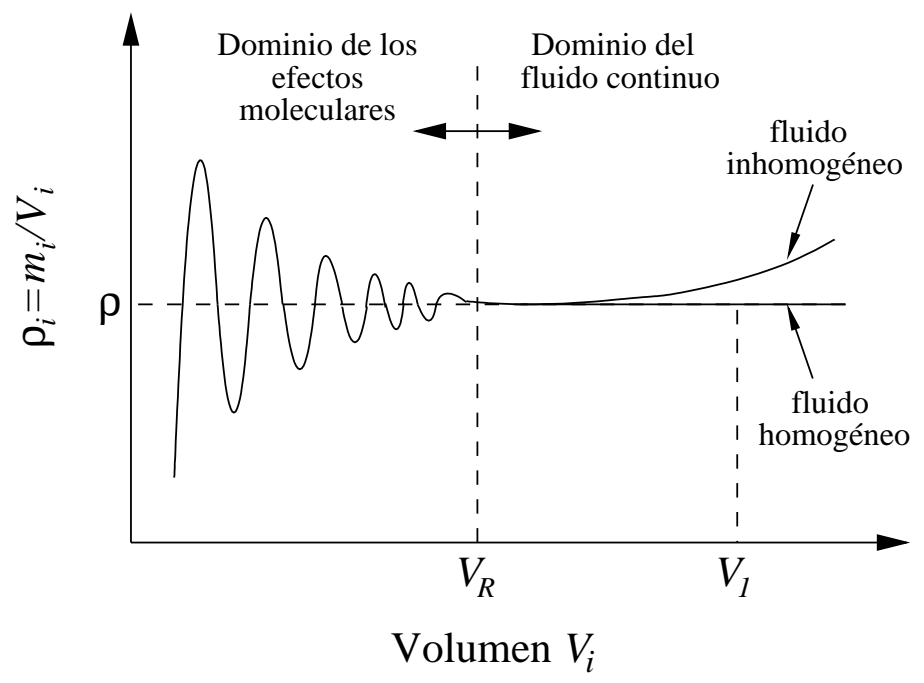

Figura 2.1: Curva teórica de densidad en función del volumen para un medio fluido (adaptada de [7]).

$\rho_{i}$ con $V_{i}$, que se mantiene hasta cierto valor $V_{R}$. Sin embargo, a medida que $V_{i}$ se hace más pequeño $\left(V_{i}<V_{R}\right)$, el cociente $m_{i} / V_{i}$ comienza a ser apreciablemente afectado. Esto ocurre cuando la longitud característica de $V_{i}$ se hace del orden de magnitud de la distancia media entre las moléculas (camino libre medio de las moléculas). A medida que $V_{i} \rightarrow 0$ se observan fluctuaciones muy bruscas en $m_{i} / V_{i}$ y la definición de $\rho_{i}$ pierde significado. Luego, la densidad del fluido en $P$ se puede definir como:

$$
\rho(P)=\lim _{V_{i} \rightarrow V_{R}} \rho_{i}=\lim _{V_{i} \rightarrow V_{R}} \frac{m_{i}}{V_{i}} .
$$

El volumen representativo $V_{R}$ es llamado punto físico (o punto material) del fluido en el punto matemático $P$, y puede ahora ser identificado como el volumen de una partícula en $P$. Mediante este procedimiento, un material constituido por un conjunto de moléculas en un vacío es reemplazado por un continuo que ocupa y llena todo el espacio. Obtenemos un medio físico suave, llamado fluido, para cada punto del cual está definida una densidad continua $\rho(x, y, z)$. En el caso de tratar con un fluido homogéneo, $\rho(x, y, z)$ será una función constante, mientras que si el fluido es inhomogéneo, la misma registrará cambios más o menos suaves dependiendo del grado de inhomogeneidad.

Knudsen [50] define un número adimensional $K_{n}=\lambda / L$ (llamado usualmente número de Knudsen) donde $\lambda$ es la distancia media entre moléculas y $L$ la longitud característica donde no ocurren cambios macroscópicos de la densidad. Cuando $K_{n}<0.01$ el fluido 
puede considerarse como un continuo para el cual pueden aplicarse las condiciones de medio continuo (por ejemplo, en la forma de ecuaciones diferenciales en derivadas parciales). Este número entonces establece los límites para $V_{R}$. Cuando $K_{n} \simeq 1$ tenemos un régimen de flujo caótico, y cuando $K_{n}>1$ tenemos un flujo molecular libre o flujo de Knudsen.

La aproximación continua nos ha permitido pasar del nivel molecular al tratamiento continuo de fenómenos físicos que involucran a los fluidos. Recordemos que se ha tratado con fluidos únicamente y que aún no hemos puesto atención al medio en el cual estos fluyen. Tenemos entonces un medio continuo (el fluido) limitado por superficies sólidas (las superficies sólidas del medio poroso). En cada punto (dentro del medio fluido) podemos definir las propiedades dinámicas y cinemáticas de la partícula utilizando la aproximación del continuo. Para poder resolver el problema del flujo a este nivel o escala de observación, es preciso resolver las ecuaciones de Navier-Stokes [13]. De ser esto posible, dispondríamos de una descripción a escala microscópica del flujo del fluido en el medio poroso. Sin embargo, para resolver estas ecuaciones es necesario contar con condiciones de borde para la velocidad del fluido en la interfase fluido-sólido. Esto es prácticamente imposible, excepto en casos especialmente simples, como por ejemplo un medio conceptual compuesto de tubos capilares rectos. Además, no es fácil definir las condiciones de borde puesto que sería preciso describir con exactitud la geometría interna del medio poroso. Consecuentemente, cualquier intento por resolver el problema de flujo a escala microscópica queda prácticamente descartado.

De lo expuesto se puede concluir que la manera obvia de sortear estas dificultades es pasar a una escala mayor de promediación (nivel macroscópico o escala macroscópica). Esto es nuevamente una aproximación continua, pero a una escala mayor que la establecida para el fluido. En lo que sigue trataremos con la aproximación macroscópica de la dinámica de fluidos en medios porosos.

\subsubsection{Porosidad y Volumen Representativo Elemental}

En la sección anterior hemos visto que los conceptos de partícula (o punto físico) y de volumen sobre el cual las propiedades físicas son promediadas, son esenciales para comprender el concepto de medio continuo. Esto también es cierto en lo referente al paso del nivel microscópico al macroscópico. Nuestra tarea es entonces, determinar el tamaño del volumen representativo de un medio poroso en torno de un punto $P$. Sabemos que 
este volumen debería ser mucho más pequeño que la escala del problema, puesto que de otro modo la promediación resultante no representaría lo que ocurre en $P$. Por otro lado debe ser suficientemente mayor al tamaño de un poro de manera que incluya un número de poros tal que la promediación estadística sea representativa del medio poroso.

Cuando el medio poroso es inhomogéneo, es decir, con porosidad variable en el espacio, el límite superior para la dimensión espacial del volumen representativo debe ser una longitud característica que indique el rango dentro del cual los cambios de porosidad tienen lugar. El límite inferior casi siempre está relacionado con el tamaño de los poros o de los granos.

Definimos entonces lo que se entiende por porosidad volumétrica y por volumen representativo elemental (REV) asociado a dicha porosidad, siguiendo el mismo procedimiento que se describió anteriormente para definir la densidad del fluido. Para definir el REV utilizamos el concepto de porosidad, ya que se trata de una propiedad básica de una matriz porosa. Notar que en cierto sentido, la porosidad es el equivalente a la densidad (masa por unidad de volumen).

Sea $P$ un punto matemático dentro del dominio ocupado por el medio poroso. Consideremos un volumen $V_{i}$ (supongamos, por ejemplo, que tiene la forma de una esfera) de dimensiones mucho mayores que las de un único grano o poro, para el cual $P$ es el centroide. Para este volumen se determina el siguiente cociente:

$$
\phi_{i}=\phi_{i}\left(V_{i}\right)=\left(V_{p}\right)_{i} / V_{i}
$$

donde $\left(V_{p}\right)_{i}$ es el volumen del espacio poral dentro de $V_{i}$. Repitiendo el mismo procedimiento, puede obtenerse una secuencia de valores $\phi_{i}(i=1,2,3, \ldots)$ a partir de la disminución del tamaño de $V_{i}$ en torno de $P$. La Fig. 2.2 muestra en forma esquemática el resultado de este cálculo. Para valores grandes de $V_{i}$, la razón $\phi_{i}$ podrá atravesar cambios graduales a medida que el volumen disminuye, especialmente cuando el dominio considerado es inhomogéneo. Debajo de un cierto valor de $V_{i}$, estos cambios o fluctuaciones tienden a decaer, presentando solo pequeñas variaciones que se deben a la distribución aleatoria de tamaños porales en las vecindades de $P$. Sin embargo, para valores de $V_{i}$ inferiores a cierto valor $V^{*}$ se observan bruscas fluctuaciones en el valor de $\phi_{i}$. Esto ocurre cuando las dimensiones del volumen se aproximan a las dimensiones porales. Finalmente, cuando $V_{i} \rightarrow 0$ convergiendo al punto matemático $P, \phi_{i}$ se convertirá en 1 o 0 dependiendo de si $P$ está dentro de un poro o dentro de la matriz sólida del medio. 


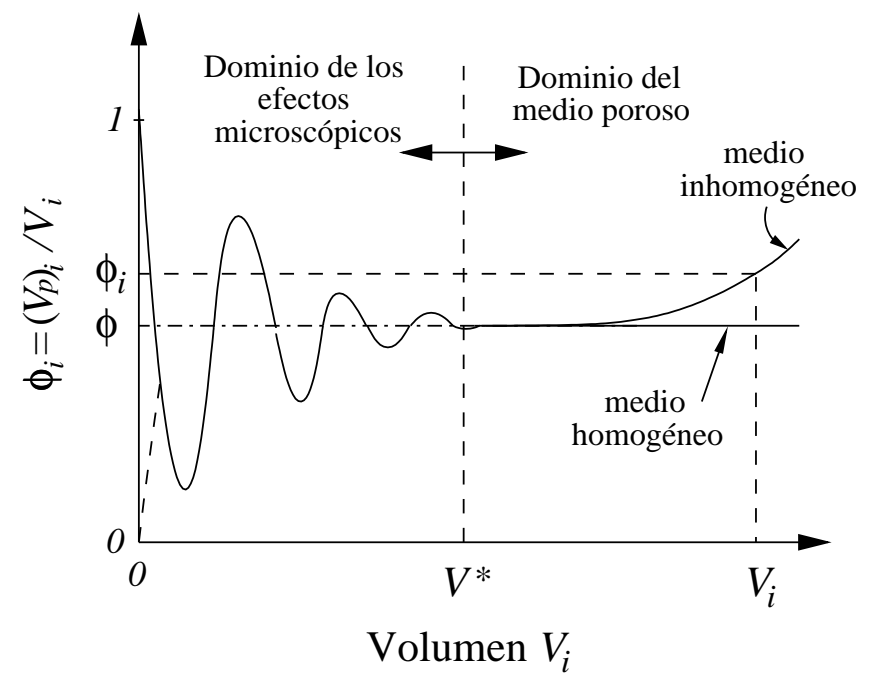

Figura 2.2: Curva teórica de porosidad en función del volumen para un medio poroso (adaptada de [7]).

La porosidad volumétrica $\phi(P)$ del medio en el punto $P$ está definida como el límite de la razón $\phi_{i}$ cuando $V_{i} \rightarrow V^{*}$ :

$$
\phi=\lim _{V_{i} \rightarrow V^{*}} \phi_{i}=\lim _{V_{i} \rightarrow V^{*}} \frac{\left(V_{p}\right)_{i}}{V_{i}} .
$$

Para valores de $V_{i}<V^{*}$, debemos considerar la presencia de poros y partículas sólidas; en este rango no hay un valor que represente la porosidad en $P$. El volumen $V^{*}$ es entonces el Volumen Representativo Elemental (REV) del punto físico o material del medio poroso en el punto matemático $P$, que por simplicidad lo designaremos con la letra $V$.

En síntesis, introduciendo el concepto de porosidad y la definición de REV, hemos reemplazado el medio real por un medio continuo ficticio, para el cual podemos asignar valores de cualquier propiedad media (ya sea del medio o de los fluidos que llenan el espacio poral) a un punto matemático del espacio ocupado por dicho medio.

\subsection{Variables macroscópicas}

Para describir el flujo de agua bajo la aproximación del continuo, además de la porosidad del REV definida anteriormente, resulta necesario definir otras variables macroscópicas que junto con esta caracterizan por completo el fenómeno. Estas variables no solo representan propiedades intrínsecas del medio poroso, sino que también describen la interacción de este con los fluidos que ocupan los espacios porales. 
Para introducir las variables consideremos un volumen representativo elemental de medio poroso $V$ :

$$
V=V_{s}+V_{p}
$$

siendo $V_{s}$ y $V_{p}$ los volúmenes de la matriz sólida y de los poros, respectivamente. Si se supone que el espacio poral está completamente ocupado por agua y aire, valdrá:

$$
V_{p}=V_{w}+V_{a}
$$

donde $V_{w}$ y $V_{a}$ son los volúmenes de agua y aire, respectivamente ${ }^{2}$.

La saturación (o grado de saturación) de un fluido se define como la fracción de volumen de poros que está ocupado por dicho fluido. En el caso de un medio parcialmente saturado definimos entonces las saturaciones de agua $S_{w}$ y de aire $S_{a}$ como:

$$
S_{w}=\frac{V_{w}}{V_{p}}, \quad S_{a}=\frac{V_{a}}{V_{p}},
$$

de modo que

$$
S_{w}+S_{a}=1
$$

De acuerdo con (2.6), $S_{w}$ y $S_{a}$ pueden tomar cualquier valor en el intervalo [0,1]. Sin embargo, en medios porosos reales este intervalo es más acotado. Se ha comprobado experimentalmente que no es posible extraer de una dada muestra todo su contenido de agua. Esto puede deberse al bloqueo de los canales de flujo o a una fuerte adsorción de la fase sólida. Por esta razón la saturación de agua resulta siempre mayor o igual que un valor mínimo $S_{w, r}$, llamado saturación residual de agua. Asimismo, no es posible que el volumen poral completo esté ocupado por la fase líquida, puesto que existe una pequeña fracción de aire que permanece entrampada y no puede ser desplazada por el agua. Este hecho impone un límite superior $S_{w, s}$, llamado satruración máxima de agua. Luego, el rango de variación de $S_{w}$ puede expresarse del siguiente modo:

$$
0<S_{w, r} \leq S_{w} \leq S_{w, s}<1
$$

Por razones prácticas se suele utilizar la saturación efectiva de agua $S_{e, w}$, la cual se define del siguiente modo:

$$
S_{e, w}=\frac{S_{w}-S_{w, r}}{S_{w, s}-S_{w, r}}
$$

\footnotetext{
${ }^{2}$ Se utilizarán los subíndices $w$ y a del inglés water y air, respectivamente.
} 
Como se desprende de la ecuación anterior, $S_{e, w}$ está limitada al intervalo [0,1] para valores de $S_{w}$ en el intervalo $\left[S_{w, r}, S_{w, s}\right]$.

La saturación efectiva de aire $S_{e, a}$ se obtiene a partir de $S_{a}$ siguiendo un razonamiento análogo. Definiendo la saturación residual de aire $S_{a, r}=1-S_{w, s}$ y la saturación máxima de aire $S_{a, s}=1-S_{w, r}$, se tiene:

$$
S_{e, a}=\frac{S_{a}-S_{a, r}}{S_{a, s}-S_{a, r}} .
$$

A partir de las ecuaciones (2.9) y (2.10), y en virtud de (2.7) se obtiene:

$$
S_{e, w}+S_{e, a}=1
$$

Dado que las ecuaciones (2.7) y (2.11) nos permiten vincular las saturaciones de ambas fases, especificar el valor de una de ellas es suficiente para conocer el estado de humedad del medio. Por conveniencia se eligen los valores de saturación de agua $S_{w}$ y $S_{e, w}$. De aquí en adelante, y para simplificar la notación, se utilizará el término saturación para hacer referencia a la saturación de agua $S_{w}$ y se designará con la letra $S$. Del mismo modo utilizaremos el término saturación efectiva para referirnos a la saturación efectiva de agua, y la denotaremos simplemente como $S_{e}$. No obstante ello, en el Capítulo 5 haremos uso de las saturaciones de agua y aire para derivar un modelo analítico para la descripción del flujo bifásico en rocas fracturadas.

El flujo simultáneo de agua y aire puede en principio ocurrir para cualquier valor de saturación efectiva. Sin embargo, tanto el flujo de agua como el de aire a través de una muestra se establece a partir de ciertos valores umbrales de la saturación efectiva. En un experimento de drenaje una muestra completamente saturada $\left(S_{e}=1\right)$ comienza a desaturarse mediante la inyección progresiva de aire. La saturación disminuye a causa del agua desplazada por el aire. No obstante el flujo de aire a través de la muestra no comienza a desarrollarse hasta tanto el volumen de aire inyectado sea suficiente como para que se formen canales interconectados de aire que atraviecen la muestra. La saturación efectiva para la cual comienza a desarrollarse el flujo de aire se denomina saturación efectiva de emergencia para el flujo de aire $S_{e, 0, a}$. Para todo valor $S_{e}<S_{e, 0, a}$ el aire puede fluir a través de la muestra. Por su parte, el flujo de agua puede tener lugar para cualquier valor de $S_{e}$ en un experiento de drenaje. El experimento mediante el cual una muestra completamente desaturada $\left(S_{e}=0\right)$ comienza a llenarse con agua se denomina 
imbibición. Al igual que ocurre en el drenaje, existe un valor $S_{e, 0, w}$ por debajo del cual el agua no logra formar canales de interconección que permitan el desarrollo del flujo de agua a través de la muestra. Al valor de saturación efectiva $S_{e, 0, w}$ se lo denomina saturación efectiva de emergencia para el flujo de agua. Para todo valor de $S_{e}>S_{e, 0, w}$ el agua puede fluir a través de la muestra. El flujo de aire puede tener lugar para todo valor de $S_{e}$ durante un experimento de imbibición [98, 28].

Otra de las variables comúnmente usadas en hidrología (y otras ciencias vinculadas) para cuantificar el contenido de humedad del suelo es el contenido volumétrico de agua $\theta$. Esta variable se define como la fracción total del volumen de suelo que está ocupada por agua, esto es:

$$
\theta=\frac{V_{w}}{V}
$$

La relación entre la saturación $S$ y el contenido volumétrico $\theta$ es:

$$
\theta=\frac{V_{w}}{V}=\frac{V_{p}}{V} \frac{V_{w}}{V_{p}}=\phi S,
$$

siendo $\phi$ la porosidad, definida en la sección anterior. Teniendo en cuenta la relación (2.13), el rango de variación de $\theta$ puede establecerse del siguiente modo:

$$
\theta_{r} \leq \theta \leq \theta_{s}
$$

siendo $\theta_{r}=\phi S_{r}$ el contenido residual de agua y $\theta_{s}=\phi S_{s}$ el contenido máximo de agua dentro del REV.

En muchos casos resulta útil expresar el estado de humedad en términos del contenido efectivo de agua $\theta_{e}$, cuyo valor numérico es igual a la saturación efectiva $S_{e}$ :

$$
\theta_{e}=\frac{\theta-\theta_{r}}{\theta_{s}-\theta_{r}}=\frac{S-S_{r}}{S_{s}-S_{r}}=S_{e}
$$

siendo sus rangos de variación $0 \leq \theta_{e}, S_{e} \leq 1$.

Las ecuaciones que describen el flujo de agua suelen expresarse en términos de la altura hidráulica $H$. Esta altura se obtiene al considerar la ecuación de Bernoulli en un fluido no viscoso e incompresible sobre el que solo actúa la fuerza de gravedad. La altura hidráulica $H$ en un punto $P(x, y, z)$ situado en un sistema de referencia arbitrario cuyo eje de coordenadas $z$ coincide con la dirección de la gravedad y su sentido es positivo hacia arriba, se define como [63]:

$$
H=h_{p}+h_{u}+z
$$


donde

$$
\begin{gathered}
h_{p}=\frac{p_{w}}{\rho g}=\text { altura de presión, } \\
h_{u}=\frac{u^{2}}{2 g}=\text { altura de velocidad, }
\end{gathered}
$$

siendo $p_{w}$ la presión del agua, $\rho$ su densidad, $g$ la aceleración de la gravedad y $u$ la velocidad del fluido. Al igual que en la Ecuación de Bernoulli, cada uno de los términos de la ecuación (2.16) puede ser interpretado en términos de energía.

Teniendo en cuenta que las velocidades dentro de un medio poroso son en general muy bajas se suele desestimar el término de velocidad $h_{u}$ y considerar la altura hidráulica simplemente como:

$$
H=h+z,
$$

donde se ha utilizado el símbolo $h$ para simplificar la notación de la altura de presión $h_{p}$.

Otra de las variables que se utiliza con frecuencia, tanto en la práctica como en desarrollos teóricos, es la presión capilar $p_{c}$. Si bien su definición tiene origen en el estudio a escala microscópica, puede asimismo interpretarse como una cantidad macroscópica. En un medio parcialmente saturado, la interfase aire-agua constituye una discontinuidad de presión. La presión del aire $p_{a}$ es superior a la presión de agua $p_{w}$, y la magnitud de la curvatura de la interfase resulta proporcional a la diferencia entre estas presiones. Se define entonces la presión capilar $p_{c}$ como:

$$
p_{c}=p_{a}-p_{w}
$$

La relación entre $p_{c}$ y la curvatura de la interfase aire-agua se determina planteando el equilibrio dinámico en un área elemental en torno de un punto situado en dicha interfase (ver Fig. 2.3). Si $r^{\prime}$ y $r^{\prime \prime}$ son los radios principales de curvatura de la superficie, se obtiene la siguiente relación [63]:

$$
p_{c}=\sigma_{w a}\left(\frac{1}{r^{\prime}}+\frac{1}{r^{\prime \prime}}\right)=\frac{2 \sigma_{w a}}{r},
$$

donde $r$ es el radio de curvatura medio de la superficie y $\sigma_{w a}$ es la tensión superficial. Esta última se define como la cantidad de trabajo necesaria para separar un área unitaria de la interfase de los dos fluidos. La ecuación (2.21) es conocida como Ecuación de Laplace para la presión capilar [7].

Aunque las magnitudes que intervienen en la ecuaciones (2.20) y (2.21) han sido definidas a partir de conceptos físicos aplicables a escala microscópica, suelen utilizarse 


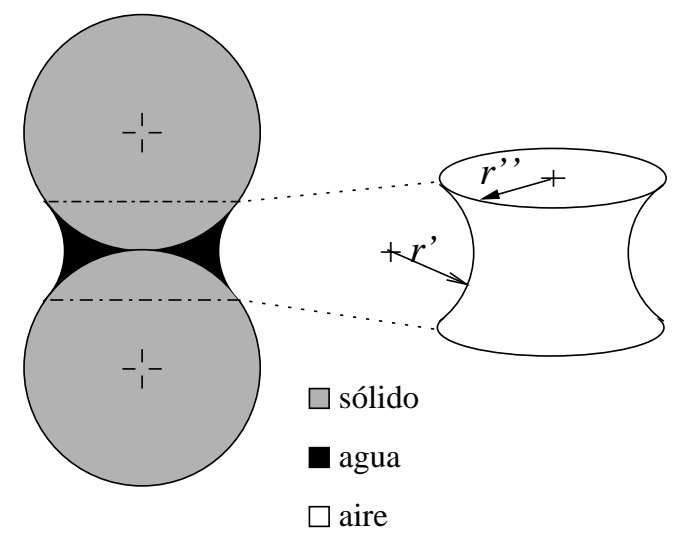

Figura 2.3: Curvatura de la interfase aire-agua.

como cantidades macroscópicas representativas de un dado volumen de medio poroso. Esto significa que podrían obtenerse a partir de promediar sus valores sobre todo el volumen considerado. Sin embargo, dada la extrema complejidad de la geometría interna de un medio poroso, resulta imposible determinar estas variables a partir de mediciones directas. En su lugar se suelen adoptar modelos conceptuales simplificados para representar la geometría interna del espacio poral. Un ejemplo típico, muy utilizado en desarrollos teóricos, es el tubo capilar recto, para el cual la presión capilar verifica:

$$
p_{c}=\frac{2 \sigma \cos (\beta)}{r}
$$

donde $\beta$ es el ángulo de contacto entre la interfase agua-aire y las paredes del tubo, $r$ es el radio del tubo y $\sigma$ es la tensión superficial entre el agua y el material del tubo.

Cuando se estudia el flujo no saturado de agua, suele suponerse que el aire se encuentra a presión constante. Si se toma como presión de referencia a la presión de aire $p_{a} \mathrm{y}$ se le asigna el valor cero, de la ecuación (2.20) se tiene que:

$$
p_{w}=p_{a}-p_{c}=-p_{c}
$$

En este caso la presión de agua $p_{w}$ es negativa y es por eso que se la suele denominar tensión o succión. Como consecuencia, la altura de presión $h$ también será negativa (ver ecuación (2.17)) y puede interpretarse como la altura de agua que absorbe el medio poroso en el punto $P$ cuando es utilizado un tensiómetro para su medición. En cambio, si el punto $P$ está situado en un medio completamente saturado, la presión de agua $p_{w}$ se considera positiva y $h$ representa la altura de la columna de agua sobre el punto $P$ que 
se observaría al utilizar un piezómetro. En este caso se suele denominar a $h$ como altura piezométrica.

Resta definir una variable más, la conductividad hidráulica, la cual fue originalmente introducida por Darcy en su ley empírica (ley de Darcy) válida para medios porosos saturados. Posteriormente Buckingham [14] propuso una ley más generalizada que rige para medios porosos parcialmente saturados. Lo que hoy se conoce como ley de BuckinghamDarcy fue formalizada por Richards [84].

\subsection{Ley de flujo de Buckingham-Darcy}

Para enunciar la ley de flujo de Buckingham-Darcy introduciremos una nueva variable macroscópica, el vector flujo de agua q. Este vector se define como la velocidad de flujo por unidad de área, siendo sus unidades longitud por unidad de tiempo (volumen por unidad de área y unidad de tiempo). La ley de flujo de Buckingham-Darcy en términos de $h$ se expresa [14, 84]:

$$
\mathbf{q}=-\boldsymbol{K}(h) \cdot \boldsymbol{\nabla}(h+z),
$$

donde $\boldsymbol{K}(h)$ es el tensor de conductividad hidráulica, cuyas unidades son las mismas que las de q. La expresión de la ley es análoga a la ley de Darcy, con la salvedad de que $\boldsymbol{K}$ es una función de $h$. La conductividad hidráulica suele expresarse como:

$$
\boldsymbol{K}(h)=\boldsymbol{K}_{\boldsymbol{s}} K_{r}(h),
$$

donde $\boldsymbol{K}_{\boldsymbol{s}}$ es la conductividad hidráulica saturada y $K_{r}(h)$ (adimensional) es la conductividad hidráulica relativa, función que depende del estado de saturación y de las propiedades físicas del medio poroso. La función $K_{r}(h)$ es positiva y alcanza su valor máximo $K_{r}(h)=1$ cuando el medio está saturado, es decir para valores positivos de $h$. En este último caso la ecuación (2.24) coincide con la ley de Darcy. En muchos trabajos aplicados al caso saturado suele ser común utilizar la permeabilidad $\boldsymbol{\kappa}$, la cual está definida en términos de $\boldsymbol{K}_{\boldsymbol{s}}$ como:

$$
\boldsymbol{\kappa}=\frac{\mu \boldsymbol{K}_{s}}{\rho g}
$$

donde $\mu$ es la viscosidad del agua. La permeabilidad es una medida de la capacidad del medio para transmitir fluido, depende de las propiedades físicas del medio poroso pero es independiente del fluido saturante. Por esta razón se la suele llamar permeabilidad 
intrínseca. La permeabilidad tiene unidades de longitud al cuadrado, y suele expresarse en $\operatorname{Darcy}\left(1 D \simeq 10^{-12} m^{2}\right)$.

En este trabajo trataremos con medios isótropos, por lo que la conductividad hidráulica podrá caracterizarse por una magnitud escalar $K$. La misma podrá variar punto a punto en los medios que se estudien, pero la dirección del flujo será la correspondiente al gradiente de $h+z$. En este caso, la ecuación (2.24) podrá expresarse:

$$
\mathbf{q}=-K(h) \boldsymbol{\nabla}(h+z)=-K_{s} K_{r}(h) \nabla(h+z) .
$$

Vale aclarar que la ley de flujo de Buckingham-Darcy es válida siempre y cuando el flujo pueda considerarse laminar. Esto suele establecerse mediante el número de Reynolds $R_{e}$, definido como [7]:

$$
R_{e}=\frac{q \rho d}{\mu},
$$

donde $d$ es una longitud característica asociada generalmente al diámetro medio de los granos que conforman el medio poroso. La ley de Buckingham-Darcy es válida para valores de $R_{e}$ inferiores a un valor promedio comprendido entre 1 y 10 , lo cual suele verificarse en casi la totalidad de las aplicaciones al estudio del flujo en medios porosos.

\subsubsection{Flujo bifásico}

La ley de Buckingham-Darcy (2.27) puede generalizarse al estudio del flujo inmiscible de agua y aire. En este caso, el flujo de cada fase estará regido por una ley análoga que suele expresarse en términos de la permeabilidad, la viscosidad y la presión del fluido:

$$
\mathbf{q}_{\alpha}=-\frac{\kappa_{\alpha}\left(p_{\alpha}\right)}{\mu_{\alpha}} \nabla\left(p_{\alpha}+\rho_{\alpha} g z\right), \quad \alpha=w, a
$$

donde el subíndice $\alpha$ refiere a la fase considerada. La magnitud $\kappa_{\alpha}$ es la permeabilidad de la fase $\alpha$, y depende de la presión del fluido. La permeabilidad relativa de la fase $\alpha$ se define en términos de la permeabilidad como:

$$
\kappa_{r, \alpha}\left(p_{\alpha}\right)=\kappa_{\alpha}\left(p_{\alpha}\right) / \kappa
$$

Estas definiciones serán utilizadas en el Capítulo 5 para derivar un modelo constitutivo bifásico para rocas fracturadas. 


\subsection{Ecuación de Richards}

La ecuación que gobierna el flujo de agua en un medio poroso de saturación variable bajo la hipótesis de que el fluido que ocupa el espacio poral es bifásico (aire y agua) y que la presión del aire permanece constante e igual a la presión atmosférica, es la ecuación de Richards [84]. El hecho de que la presión del aire permanezca constante hace que el problema se centre exclusivamente en la fase líquida. Al flujo de agua bajo estas condiciones se lo denomina flujo no saturado.

La ecuación de Richards se obtiene de combinar la ecuación de conservación de la masa de agua con la ley de Buckingham-Darcy (2.24):

$$
\frac{\partial \theta(h)}{\partial t}-\nabla \cdot(K(h) \nabla(h+z))=F(h)
$$

Esta ecuación es válida siempre y cuando el medio poroso pueda considerarse indeformable y la densidad del agua constante. Estas condiciones se verifican en la mayoría de las aplicaciones al estudio del flujo no saturado. En el Capítulo 6 se abordará el problema del flujo en un acuífero confinado. Como se verá, es necesario asumir que el medio puede deformarse como respuesta a las variaciones de presión de agua.

El término $F(h)$ de la ecuación (2.31) representa una fuente o sumidero de agua. Este término tiene en cuenta el aporte o extracción externo de agua al medio poroso. En los casos estudiados en esta Tesis no existirá tal tipo de aporte/extracción de fluido, con lo cual $F(h)=0$. Vale sin embargo aclarar a título informativo que en numerosos casos en que la ecuación de Richards es aplicable, suelen intervenir fuentes o sumideros de agua. Un ejemplo típico es el caso en que se quiere modelar el flujo de agua en un suelo en el que existen pérdidas de agua por transpiración de las plantas [22].

Para resolver la ecuación (2.31) es necesario conocer la dependencia funcional de $\theta$ y $K$ con la altura de presión $h$. Las relaciones $\theta(h)$ y $K(h)$ son las llamadas relaciones constitutivas y son características distintivas de cada tipo de medio poroso. En la sección siguiente se hará una breve descripción de las técnicas experimentales de laboratorio para la determinación de $\theta(h)$ y $K(h)$.

\subsection{Determinación experimental de las relaciones constitutivas}

Como ya se ha mencionado, para modelar el flujo no saturado en un medio poroso se debe resolver la ecuación (2.31), para lo cual es indispensable conocer las relaciones 


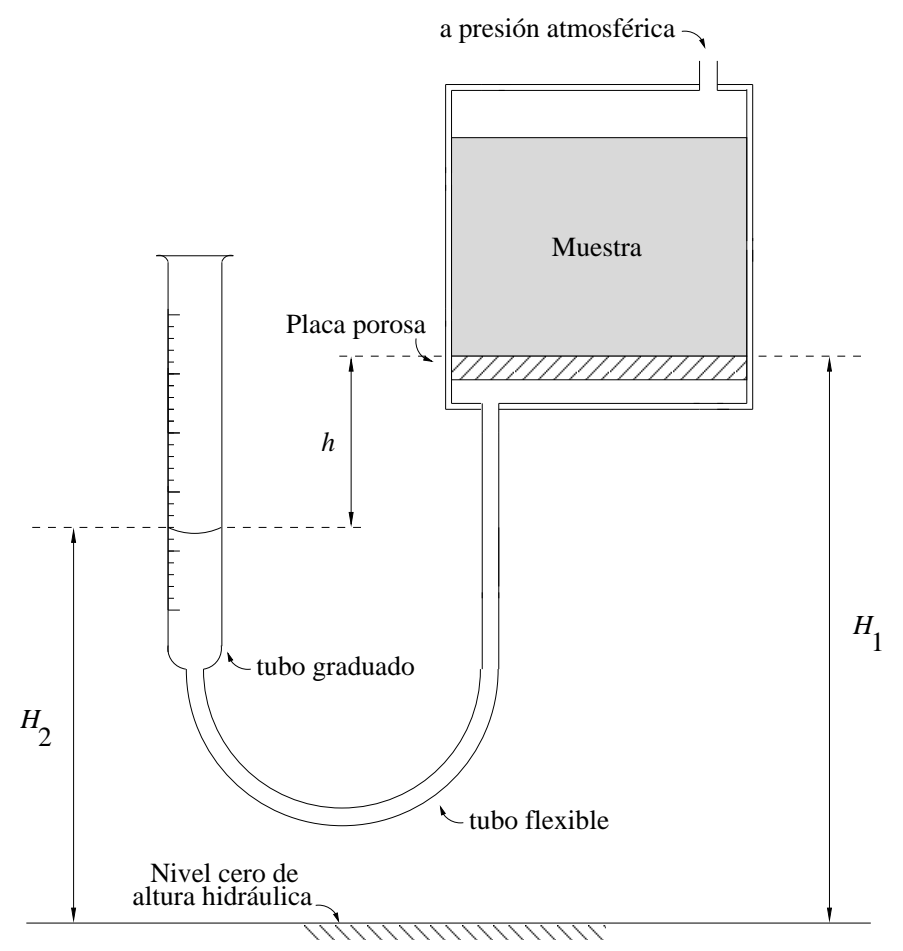

Figura 2.4: Principio de medición en laboratorio del contenido de agua en función de la altura de presión.

constitutivas $\theta(h)$ y $K(h)$ del medio considerado. Tanto $\theta(h)$ como $K(h)$ pueden medirse mediante experimentos de laboratorio realizados sobre muestras representativas de rocas. Con el propósito de comprender los principios de medición de las relaciones constitutivas, en esta sección se presentarán los métodos clásicos para su determinación experimental.

\subsubsection{Determinación de la curva de contenido de agua}

La Fig. 2.4 esquematiza un procedimiento de laboratorio utilizado para medir el contenido de agua para un determinado valor de altura de presión $h$ [31]. Una muestra de roca completamente saturada se coloca sobre una placa porosa en el interior de un recipiente impermeable. La placa porosa se encuentra saturada y está conectada mediante un tubo flexible con una columna de agua que termina en un tubo graduado. El tubo flexible permite cambiar la posición del tubo graduado para disminuir la altura de presión de agua (aumentar su valor absoluto). Cuando la altura de presión se hace más negativa, el agua drena desde la muestra, y la cantidad es medida en el tubo una vez alcanzado el equilibrio. Debe tenerse el recaudo de evitar la evaporación del agua, ya sea desde la muestra como 
desde el tubo, de lo contrario debería considerarse un sumidero externo $F(h)$. Con el objeto de obtener una curva de contenido de agua, se debe realizar un número de observaciones a alturas de presión progresivamente más negativas. Sin embargo existe un límite práctico más allá del cual la placa porosa no puede soportar mayores incrementos de tensión. Para algunas muestras este límite coincide aproximadamente con el valor respecto del cual un posterior aumento de $h$ no drena más agua hacia el exterior de la misma, límite que suele llamarse de secado. Para medios en los que el límite de secado está en el rango de -300 a -15000 cm de agua, se suele utilizar una placa de presión. En este caso, la muestra es colocada sobre una placa porosa saturada y a su vez en una cámara de presión. La presión por debajo de la placa es mantenida constante e igual a la presión atmosférica, y la presión sobre la placa porosa puede variarse entre $0.3 \mathrm{~atm}$ (-300 cm de agua) y 15 atm (-15000 cm de agua). La presión a través de la muestra hace que el agua fluya, pasando por la placa porosa y almacenándose en un recipiente que se encuentra por debajo de la cámara de presión.

\subsubsection{Determinación de la curva de conductividad hidráulica}

Un dispositivo típico utilizado en la medición de la conductividad hidráulica se muestra en forma esquemática en la Fig. 2.5 [49]. Para realizar la medición es necesario establecer sobre la muestra un flujo estacionario. La muestra se sitúa en el interior de un recipiente impermeable entre dos placas porosas. La utilización de reservorios de nivel fijo permite que se desarrolle el flujo de agua a través de la muestra, manteniendo constantes los valores de altura hidráulica en ambos extremos $\left(H_{1} \mathrm{y} H_{2}\right)$. Los tensiómetros $T_{1}$ y $T_{2}$ permiten medir la diferencia de altura de presión entre dos puntos separados una distancia L. A partir de la ley de Buckingham-Darcy aplicada al caso de flujo vertical (unidimensional) es posible plantear la siguiente relación:

$$
K(\bar{h})=\frac{q}{\left(H_{3}-H_{4}\right) / L},
$$

donde $q$ es el flujo a través de la muestra y $H_{3}$ y $H_{4}$ son las alturas hidráulicas en los tensiómetros $T_{1}$ y $T_{2}$, respectivamente. El valor de $K$ dado por la ecuación (2.32) se corresponde con la altura de presión media $\bar{h}$ definida por [49]:

$$
\bar{h}=\rho_{1} \frac{m}{\rho}+\frac{h_{3}+h_{4}}{2},
$$




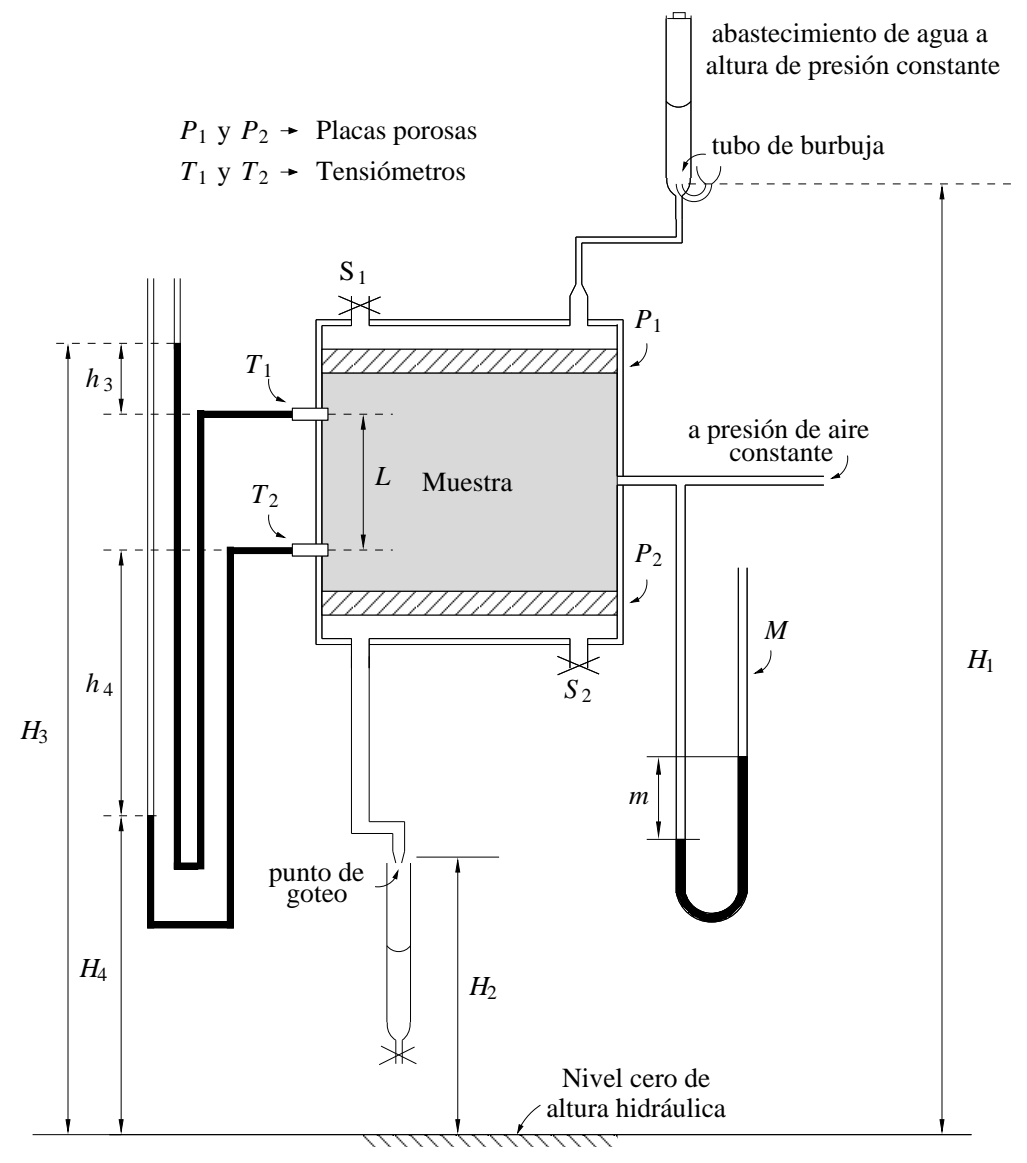

Figura 2.5: Diagrama del dispositivo de medición de $K(h)$ mediante el método de flujo estacionario (adaptada de [7]). 
siendo $\frac{\rho_{1}}{\rho} m$ la presión del aire (expresada como una columna de agua de longitud $m$ ), y $\rho_{1}$ y $\rho$ las densidades del fluido del manómetro $M$ y del agua, respectivamente. El grado de saturación de la muestra puede cambiarse sometiéndola a una presión de aire controlada (mayor a la atmosférica) que puede establecerse en términos de la altura $m$ medida en el manómetro. Comenzando las mediciones cerca de la saturación y estableciendo un número de flujos estacionarios es posible obtener pares de valores $K$ y $\bar{h}$, completando de esta manera la determinación de la conductividad hidráulica para el rango de alturas de presión abarcado por el experimento.

Los experimentos presentados en esta sección son solo algunos de lo procedimientos clásicos para la determinación de las relaciones constitutivas en muestras de roca. Aunque en la actualidad existen dispositivos más complejos que permiten obtener mediciones más confiables, los dispositivos descritos son suficientes para comprender los fundamentos físicos sobre los cuales se basan las mediciones de las relaciones constitutivas. En particular, el dispositivo presentado para la medición de $K(h)$ establece los principios para el diseño de experimentos numéricos, como veremos en el Capítulo 3.

Por último, cabe resaltar que la determinación de la conductividad hidráulica es siempre más dificultosa que la determinación del contenido de agua ya que presenta grandes limitaciones prácticas. Por ello, disponer de datos experimentales de conductividad hidráulica es poco frecuente en la mayoría de los casos. Una manera de resolver este inconveniente consiste en estimar la conductividad hidráulica a partir de los datos obtenidos para la curva de contenido de agua mediante fórmulas predictivas, como se verá en la sección siguiente.

\subsection{Fórmulas predictivas y modelos constitutivos}

Como alternativa a la medición directa de la conductividad hidráulica se han desarrollado métodos indirectos basados en relaciones entre la saturación y la presión capilar o entre la saturación y la distribución del tamaño poral. Estos métodos asumen un modelo conceptual de medio poroso para el cual el flujo puede determinarse de manera exacta. En lo que sigue se describirán las fórmulas de Burdine [15] y de Mualem [73]. Ambas fórmulas han sido utilizadas ampliamente en el desarrollo de modelos constitutivos para medios porosos. 

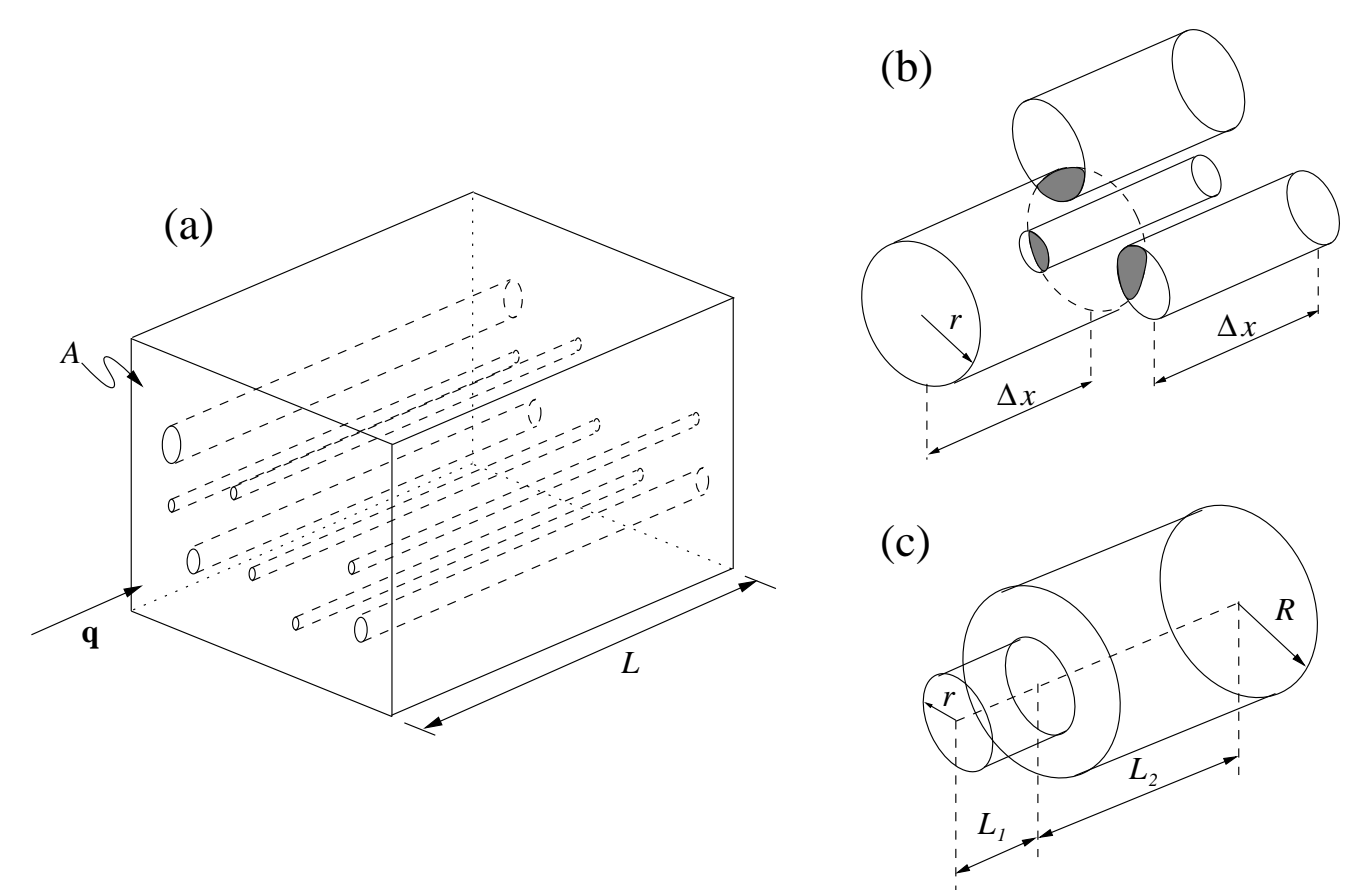

Figura 2.6: Modelo de tubos capilares rectos (a) y geometría poral de los modelos de Burdine (b) y Mualem (c).

\subsubsection{Fórmula de Burdine}

El desarrollo que se presenta a continuación sigue las ideas del trabajo de Wyllie y Gardner [111]. Si bien el mismo permite arribar a la fórmula de Burdine, su deducción matemática es diferente a la presentada originalmente por este último [7] .

El modelo conceptual supone que la muestra de roca está inicialmente compuesta por un conjunto de tubos capilares rectos de sección circular, como se muestra en la Fig. 2.6 (a). Los radios de los tubos están comprendidos en el rango $r_{1}<r<r_{2}$, y su distribución está dada por una función $f(r)$ tal que $f(r) d r$ representa el número de tubos cuyos radios se encuentran en el intervalo $(r, r+d r)$. Según la definición de $f$, la porosidad $\phi$ de la muestra puede calcularse como:

$$
\phi=\frac{1}{A} \int_{r_{1}}^{r_{2}} \pi r^{2} f(r) d r
$$

siendo $A$ el área de la muestra, transversal a la dirección del flujo q (ver Fig. 2.6 (a)).

Para que el modelo de tubos capilares representado en la Fig. 2.6 (a) sea más realista, se procede a cortar la muestra en placas delgadas de espesor $\Delta x$, transversales a la dirección de flujo. En cada una de las placas se reordenan los segmentos de tubos capilares 
de manera aleatoria, para luego ensamblar la muestra, dando como resultado arreglos más complejos, como el que se muestra en la Fig. 2.6 (b). Suponiendo que la presión capilar $p_{c}$ es constante en todas las placas (y por lo tanto en la muestra) se asume que el agua ocupa todos los tubos de radio menor o igual que un valor $r^{\prime}$ definido por:

$$
r^{\prime}=2 \sigma / p_{c}
$$

mientras que todos los tubos de radio mayor que $r^{\prime}$ se encuentran ocupados por aire. Con este criterio de ocupación, la saturación efectiva $S_{e}^{\prime}=S_{e}\left(r^{\prime}\right)$ vendrá dada por:

$$
S_{e}^{\prime}=\frac{\int_{r_{1}}^{r^{\prime}} \pi r^{2} f(r) d r}{\int_{r_{1}}^{r_{2}} \pi r^{2} f(r) d r}
$$

Nótese que las ecuaciones (2.35) y (2.36) establecen una relación funcional entre la presión capilar y la saturación efectiva.

Diferenciando (2.36) respecto de $r^{\prime}$ y utilizando (2.34) obtenemos la siguiente ecuación que nos será de utilidad:

$$
d S_{e}(r)=\frac{\pi r^{2} f(r) d r}{\phi A}
$$

Considérese un punto cualquiera en la interfase entre dos placas contíguas. Si el punto se encuentra situado en un lugar del espacio ocupado por agua en una de las placas, la probabilidad de que dicho punto verifique lo propio en la placa contigua es $\phi S_{e}^{\prime}$. Luego, el área común a un único tubo de radio $r$ en una placa, y todos los tubos llenos de agua en la placa contígua viene dada por $\pi r^{2} \phi S_{e}^{\prime}$. Para calcular el flujo a través de dos placas sucesivas se considera entonces que cada tubo de sección $\pi r^{2}$ en una de las placas se conecta con un área $\pi r^{2} \phi S_{e}^{\prime}$ de la placa siguiente (el área sombreada en el ejemplo de la Fig. 2.6 (b)). Para representar esta transición, Wyllie y Gardner [111] utilizan un tubo capilar equivalente cuyo radio $r^{\prime \prime}$ es tal que el área de su sección verifica la siguiente relación:

$$
\lambda \pi\left(r^{\prime \prime}\right)^{2}=\pi r^{2} \phi S_{e}^{\prime}
$$

donde $\lambda(>1)$ es un coeficiente adimensional que refleja la manera en la cual está distribuida el área poral interconectada. Utilizando la ley de Hagen-Poiseuille para un tubo capilar recto de sección circular, el caudal de agua a través del tubo equivalente de radio $r^{\prime \prime}$ vendrá dado por [13]:

$$
Q_{c}=-\frac{\pi\left(r^{\prime \prime}\right)^{4}}{8 \mu} \frac{\Delta p_{w}}{L}=-\frac{\pi r^{4} \phi^{2}\left(S_{e}^{\prime}\right)^{2}}{8 \mu \lambda^{2}} \frac{\Delta p_{w}}{L},
$$


donde $\mu$ es la viscosidad del fluido y $\Delta p_{w} / L$ el gradiente de presión de agua al que se encuentra sometida la muestra. Luego, el caudal aportado por los tubos capilares de radios entre $r$ y $r+d r$ será $Q_{c}(r) f(r) d r$, y el flujo total (caudal total dividido el área de la sección transversal) será:

$$
q=\frac{\int_{r_{1}}^{r^{\prime}} Q_{c}(r) f(r) d r}{A} .
$$

Reemplazando (2.39) en (2.40), y utilizando (2.35) y (2.37) se obtiene:

$$
q=-\frac{\phi^{3}\left(S_{e}^{\prime}\right)^{2} \sigma^{2}}{2 \mu \lambda^{2}} \frac{\Delta p_{w}}{L} \int_{0}^{S_{e}^{\prime}} \frac{d S_{e}}{p_{c}^{2}\left(S_{e}\right)} .
$$

Por otra parte, la conductividad hidráulica del modelo puede calcularse utilizando la ley de Buckingham-Darcy escrita en su forma escalar y en términos del gradiente de presión de agua:

$$
q=-\frac{K}{\rho g} \frac{\Delta p_{w}}{L} .
$$

Igualando (2.42) con (2.41) obtenemos para la conductividad hidráulica $K\left(S_{e}^{\prime}\right)$ :

$$
K\left(S_{e}^{\prime}\right)=\frac{\phi^{3} \rho g\left(S_{e}^{\prime}\right)^{2} \sigma^{2}}{2 \mu \lambda^{2}} \int_{0}^{S_{e}^{\prime}} \frac{d S_{e}}{p_{c}^{2}\left(S_{e}\right)} .
$$

La conductividad hidráulica saturada se obtiene integrando sobre todo el rango de variación de $S_{e}$, es decir:

$$
K_{s}=K(1)=\frac{\phi^{3} \rho g \sigma^{2}}{2 \mu \lambda^{2}} \int_{0}^{1} \frac{d S_{e}}{p_{c}^{2}\left(S_{e}\right)} .
$$

Finalmente, la conductividad hidráulica relativa resulta del cociente $K\left(S_{e}^{\prime}\right) / K_{s}$ :

$$
K_{r}\left(S_{e}^{\prime}\right)=\left(S_{e}^{\prime}\right)^{2} \frac{\int_{0}^{S_{e}^{\prime}} \frac{d S_{e}}{p_{c}^{2}\left(S_{e}\right)}}{\int_{0}^{1} \frac{d S_{e}}{p_{c}^{2}\left(S_{e}\right)}}
$$

La ecuación (2.45) fue derivada por Burdine [15] utilizando la teoría del radio hidráulico. Notar que la ecuación (2.45) permite predecir la conductividad hidráulica relativa a partir de conocer la dependencia entre la saturación efectiva y la presión capilar, o lo que es equivalente, la saturación efectiva en función de la altura de presión.

La fórmula de Burdine fue utilizada por Brooks y Corey [13] para obtener uno de los modelos constitutivos más utilizados en la literatura. A partir de un análisis estadístico sobre un gran número de muestras de suelo, los citados autores proponen una expresión analítica empírica para $\theta(h)$, y mediante la fórmula de Burdine obtienen una expresión 
para $K(h)$. Las expresiones del modelo constitutivo de Brooks y Corey se detallan a continuación:

$$
\begin{array}{r}
\theta(h)= \begin{cases}\left(\theta_{s}-\theta_{r}\right)\left[-\alpha_{b c} h\right]^{\beta_{b c}}+\theta_{r} & h<-1 / \alpha_{b c} \\
\theta_{s} & h \geq-1 / \alpha_{b c}\end{cases} \\
K(h)= \begin{cases}K_{s}\left(-\alpha_{b c} h\right)^{-\left(2+3 \beta_{b c}\right)} & h<-1 / \alpha_{b c} \\
K_{s} & h \geq-1 / \alpha_{b c},\end{cases}
\end{array}
$$

donde $\alpha_{b c}$ es el recíproco de la presión de aire entrampado y $\beta_{b c}$ es un parámetro relacionado con la distribución de tamaño poral. Si bien estas relaciones fueron obtenidas originalmente en forma empírica, la expresión analítica de $\theta(h)$ para este modelo puede obtenerse a partir de un modelo conceptual físico basado en principios de geometría fractal [106].

\subsubsection{Fórmula de Mualem}

La fórmula predictiva de Mualem [73] asume que los poros del medio se encuentran interconectados y distribuidos en forma aleatoria en toda la muestra. Cada poro está caracterizado por su radio poral $r$, cuya distribución en un corte cualquiera de la muestra en la dirección transversal al flujo está dada por una función $f(r)$. Para un dado valor de saturación (o equivalentemente de presión capilar) el criterio de ocupación de la fase líquida es el mismo que el utilizado por Wyllie y Gardner [111] en la deducción de la fórmula de Burdine, por lo que las ecuaciones (2.35), (2.36) y (2.37) son igualmente válidas para este análisis.

Suponiendo que la muestra se separa en placas delgadas transversales a la dirección del flujo, la probabilidad de que un poro de radio comprendido entre $r$ y $r+d r$ en una de las caras esté interconectado con un poro de radio comprendido entre $R$ y $R+d R$ en la cara contígua es:

$$
a(r, R)=G\left(S_{e}, r, R\right) \pi r^{2} f(r) \pi R^{2} f(\rho) d r d R
$$

donde la función $G\left(S_{e}, r, R\right)$ tiene en cuenta la correlación espacial entre los poros de radios $r$ y $R$ para un dado valor de saturación efectiva.

Luego, para calcular el flujo a través de una placa, se asume que los poros de radios $r$ y $R$ pueden conceptualizarse como tubos capilares rectos conectados en serie, y cuyas 
longitudes respectivas $L_{1}$ y $L_{2}$ son proporcionales a sus radios tal como se ilustra en la Fig. 2.6 (c). Utilizando la ley de Hagen-Poiseuille y la ley de Buckingham-Darcy para este arreglo, Mualem demuestra que la contribución del arreglo a la conductividad hidráulica de la muestra es proporcional al producto $r R$. Aplicando además un factor de tortuosidad $T\left(S_{e}, r, R\right)$ y asumiendo que tanto $T$ como $G$ son funciones de $S_{e}$ únicamente, se obtiene para la conductividad hidráulica relativa la siguiente expresión:

$$
K_{r}\left(S_{e}^{\prime}\right)=T\left(S_{e}^{\prime}\right) G\left(S_{e}^{\prime}\right)\left[\frac{\int_{r_{1}}^{r^{\prime}} r^{3} f(r) d r}{\int_{r_{1}}^{r_{2}} r^{3} f(r) d r}\right]^{2}
$$

donde $r_{1}$ y $r_{2}$ son los radios mínimo y máximo, respectivamente, y $S_{e}^{\prime}$ es la saturación efectiva cuando todos los poros de radios $r \in\left[r_{1}, r^{\prime}\right]$ se encuentran llenos de agua. La potencia 2 en la fórmula (2.49) se debe a la hipótesis de que la configuración poral puede reemplazarse por un par de tubos capilares rectos cuyas longitudes son proporcionales a sus respectivos radios.

Mualem sugirió además reemplazar los factores de correlación $G\left(S_{e}^{\prime}\right)$ y tortuosidad $T\left(S_{e}^{\prime}\right)$ por un factor $\left(S_{e}^{\prime}\right)^{m}$. Finalmente, aplicando la ley capilar $(2.35)$ y haciendo uso de (2.37), se llega a:

$$
K_{r}\left(S_{e}^{\prime}\right)=\left(S_{e}^{\prime}\right)^{m}\left[\frac{\int_{0}^{S_{e}^{\prime}} \frac{d S_{e}}{p_{c}\left(S_{e}\right)}}{\int_{0}^{1} \frac{d S_{e}}{p_{c}\left(S_{e}\right)}}\right]^{2},
$$

que es la conocida fórmula de Mualem. Para mayor detalle de la derivación matemática se refiere al trabajo original de Mualem [73].

La fórmula de Mualem fue utilizada por van Genuchten [108] en la derivación de su conocido modelo constitutivo. Al igual que el modelo de Brooks-Corey, el modelo de van Genuchten fue diseñado para suelos homogéneos, y es considerado el modelo que mejor ajusta los datos experimentales en todo el rango de alturas de presión. Es por ello que se lo utiliza en la mayoría de los trabajos aplicados. Las relaciones que caracterizan a este modelo vienen dadas por:

$$
\begin{array}{r}
\theta(h)= \begin{cases}\frac{\left(\theta_{s}-\theta_{r}\right)}{\left[1+\left(\alpha_{v g}|h|\right)^{n}\right]^{m}}+\theta_{r} & h<0 \\
\theta_{s} & h \geq 0\end{cases} \\
K(h)= \begin{cases}K_{s} \frac{\left[1-\left(\alpha_{v g}|h|\right)^{n-1}\left[1+\left(\alpha_{v g}|h|\right)^{n}\right]^{-m}\right]^{2}}{\left[1+\left(\alpha_{v g}|h|\right)^{n}\right]^{\frac{m}{2}}} & h<0 \\
K_{s} & h \geq 0,\end{cases}
\end{array}
$$


donde $\alpha_{v g}, m$ y $n$ son parámetros del modelo. La expresión (2.52) es válida siempre y cuando se verifique la siguiente relación entre los parámetros $m$ y $n$ :

$$
m=1-\frac{1}{n}
$$

En un trabajo reciente, Guarracino [38] ha propuesto un modelo para estimar $K_{s}$ a partir del parámetro $\alpha_{v g}$. Este modelo permite estimar completamente la curva de $K(h)$ a partir de los parámetros de la curva de $\theta(h)$.

\subsection{Relaciones constitutivas para medios heterogéneos}

Las relaciones constitutivas definen y caracterizan por completo el comportamiento hidráulico de un medio poroso a escala macroscópica. Es decir que las curvas de $\theta(h)$ y $K(h)$ describen el comportamiento hidráulico de una dada muestra de medio, cuyo tamaño sea lo suficientemente grande como para garantizar la validez de la aproximación del continuo, y por tanto la aplicabilidad de la ecuación de Richards.

Los modelos constitutivos presentados en la sección anterior fueron concebidos para medios homogéneos, lo que significa que a los efectos de simular el flujo no saturado, las propiedades hidráulicas del medio se asumen constantes sobre toda la muestra.

Cuando una dada muestra de medio presenta algún tipo de heterogeneidad de escala mayor a la escala microscópica pero menor a la escala de la muestra, el fenómeno de flujo a través de la misma se torna más complejo. Regiones con heterogeneidades que presenten mayor conductividad que el medio que las contiene pueden favorecer enormemente el flujo de agua, constituyendo caminos preferenciales a través de los cuales el agua puede fluir con mayor facilidad. Un ejemplo de este tipo de medios heterogéneos son las rocas fracturadas, a las cuales dedicaremos especial atención en esta Tesis.

Durante las últimas dos décadas el estudio del flujo en rocas fracturadas ha captado la atención de muchos investigadores. Una de las razones principales radica en la búsqueda de repositorios de desechos nucleares. Se considera que una de las maneras más seguras de aislar estos desechos es depositarlos en rocas cristalinas a grandes profundidades. Sin embargo, la migración del agua subterránea podría contribuir al retorno de los radionucloides a la superficie terrestre, por lo cual es indispensable disponer de una caracterización hidráulica precisa para rocas fracturadas [10, 11, 33]. Por otra parte en muchos sistemas acuíferos confinados como el Guaraní, las rocas fracturadas participan 
en forma indirecta en el proceso de recarga y para poder evaluarla es preciso contar con relaciones constitutivas para este tipo de rocas [37].

Tanto en el caso de rocas fracturadas o medios con otro tipo de heterogeneidad (como por ejemplo suelos con macroporos) la conductividad hidráulica suele ser mayor en las heterogeneidades que en el resto del medio, produciéndose vías de canalización rápida [109]. Este flujo preferencial hace que la conductividad del medio sea muy diferente a lo que se obtendría si no existieran estas heterogeneidades. Es muy común que las fracturas de las rocas estén rellenas con sedimentos clásticos, resultado de su diagénesis o del flujo de agua a través de las mismas. Como resultado, las fracturas se comportan como un medio poroso de características diferentes a las de la roca en la que se encuentran (en general son mejores conductoras) y permiten que el agua fluya con mayor facilidad que en el resto de la roca. Si además las fracturas se encuentran interconectadas, se forman vías rápidas que modifican ampliamente el patrón de flujo. Como resultado final, la presencia de fracturas interconectadas en una roca no necesariamente muy conductora la hace buena conductora, modificando en forma radical sus características hidráulicas. Por esta razón se espera que las relaciones constitutivas de rocas fracturadas sean muy diferentes que las que correponden a un medio homogéneo.

Un método válido para obtener relaciones constitutivas en un medio poroso heterogéneo se basa en la simulación numérica del flujo no saturado en muestras sintéticas [25, 51, 59]. Si somos capaces de modelar física y geométricamente las heterogeneidades del medio, la simulación computacional nos permitiría predecir su comportamiento hidráulico y mediante el diseño de experimentos numéricos adecuados determinar sus relaciones constitutivas. En el Capítulo siguiente se describirá un método numérico para la simulación de relaciones constitutivas en medios porosos heterogéneos y se mostrará su implementación en el caso 2D. La implementación del método en medios 3D se presentará en el Capítulo 4. 
Capítulo 2. Relaciones constitutivas para flujo no saturado 


\section{Capítulo 3}

\section{Diseño de experimentos numéricos 2D}

En este Capítulo se presenta un método numérico para el cálculo de relaciones constitutivas en muestras bidimensionales de rocas. El método consiste en simular el experimento de laboratorio para la determinación de la conductividad hidráulica que fue descrito en el Capítulo 2. Para ello es necesario resolver la ecuación de Richards en estado estacionario utilizando condiciones de borde apropiadas. Se propone un método mixto de elementos finitos para la resolución del problema diferencial en muestras heterogéneas generales. Para concluir, se presentan ejemplos ilustrativos de la aplicación del método al caso particular de rocas fracturadas y medios porosos con heterogeneidades de tipo fractal.

\subsection{Introducción}

Como se mencionó anteriormente la determinación experimental de las relaciones constitutivas presenta numerosas dificultades técnicas, por lo que suele ser muy costosa. Por esta razón, los datos experimentales son en general poco frecuentes, en particular cuando se trata de rocas fracturadas [103]. Un método alternativo a la medición directa consiste en simular en forma numérica los experimentos de laboratorio para la determinación de las relaciones constitutivas. Para ello es necesario diseñar muestras sintéticas de roca capaces de describir física y geométricamente las características del medio de interés y simular el flujo no saturado bajo condiciones similares a las de los ensayos de laboratorio.

Esta alternativa numérica de cálculo de relaciones constitutivas ha sido utilizada por varios autores. Desbarats [25] aplica el método al cálculo de la conductividad hidráulica saturada en muestras sintéticas 2D y 3D que presentan heterogeneidades de tipo lognormal, utilizando un método de diferencias finitas para resolver las ecuaciones de flujo. La aplicación del método a la determinación de las relaciones constitutivas de rocas fracturadas fue tratado por Kwicklis y Healey [51] y por Liu y Bodvarsson [59]. En sus 
experimentos los citados autores simulan el flujo no saturado en muestras sintéticas bidimensionales de forma rectangular, asumiendo como condición de borde el mismo valor de altura de presión en las caras horizontales de la muestra. Esta condición de borde garantiza en cierto modo un valor constante de altura de presión en la muestra, pero limita la aplicabilidad de la técnica para valores grandes de presión.

En este Capítulo se presentará un método numérico para el cálculo de las relaciones constitutivas en medios porosos heterogéneos 2D. El método consiste en simular el experimento descrito en la sección (2.5.2), para lo cual es necesario resolver la ecuación de Richards en estado estacionario, con condiciones de borde que permitan imponer sobre la muestra un gradiente preestablecido de altura de presión. La resolución del problema diferencial se llevará a cabo mediante un método mixto de elementos finitos. Este método posee ventajas importantes respecto de los métodos Galerkin standard y los métodos de diferencias finitas, puesto que permite aproximar con igual precisión el campo de altura de presión y el de flujo, garantizando además la conservación local de la masa. Utilizando la solución del problema diferencial se obtendrán las relaciones constitutivas mediante aproximaciones numéricas relativamente sencillas. Para finalizar se ilustrará la aplicación del método propuesto al cálculo de las relaciones constitutivas en rocas fracturadas y a la estimación de la permeabilidad en medios porosos con heterogeneidades de tipo fractal.

\subsection{Planteo matemático del experimento}

Para describir matemáticamente el problema consideremos una muestra bidimensional rectangular que ocupa el dominio $\Omega=\left[0, L_{x}\right] \times\left[0, L_{z}\right]$ con frontera $\Gamma=\Gamma^{L} \cup \Gamma^{R} \cup \Gamma^{D} \cup \Gamma^{U}$ como se ilustra en la Figura 3.1. Los supraíndices indican el nombre de la cara según su ubicación respecto a los ejes coordenados ${ }^{1}$.

Para simular el flujo no saturado en las condiciones del experimento descrito en la sección (2.5.2) es necesario someter a la muestra a un gradiente hidráulico vertical constante. El mismo se establece manteniendo constantes los valores de altura de presión en las caras horizontales $\Gamma^{D}$ y $\Gamma^{U}$. Las caras verticales se mantienen impermeabilizadas, esto es, la componente horizontal de $\mathbf{q}$ se considera nula en $\Gamma^{L}$ y $\Gamma^{R}$. Si se asume que el aire se encuentra a presión atmosférica, y que es válida la aproximación del continuo en cada punto de la muestra, el flujo de agua bajo estas condiciones estará gobernado por

\footnotetext{
${ }^{1}$ del inglés Left (izquierda), Right (derecha), Down (abajo), Up (arriba).
} 


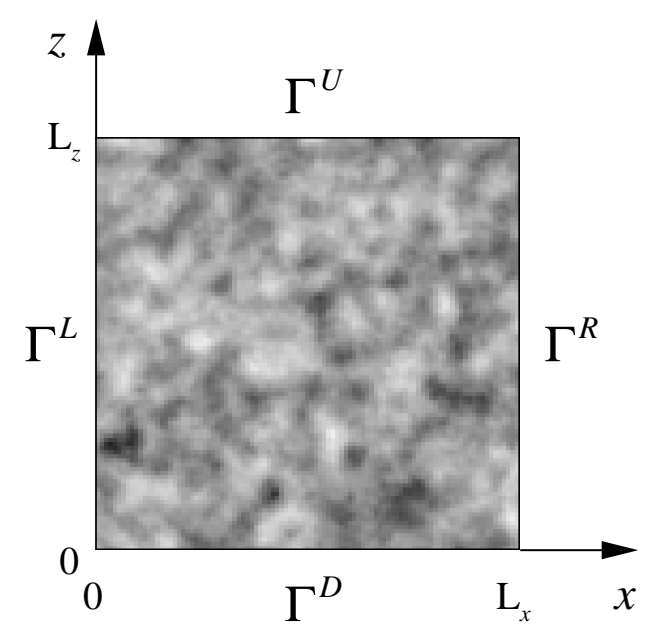

Figura 3.1: Representación matemática del dominio de simulación.

la ecuación de Richards (2.31). Dado que no existen fuentes ni sumideros dentro de la muestra $(F(h)=0)$, y que el experimento es estacionario $(\partial \theta(h) / \partial t=0)$, el problema diferencial en forma mixta puede plantearse del siguiente modo: Hallar $(h, \mathbf{q}): \Omega \times \Omega \rightarrow$ $\mathbb{R} \times \mathbb{R}^{2}$ tal que:

$$
\begin{aligned}
& \boldsymbol{\nabla} \cdot \mathbf{q}=0, \quad(x, z) \in \Omega, \\
& \mathbf{q}=-K(h) \boldsymbol{\nabla}(h+z), \quad(x, z) \in \Omega, \\
& h=h^{D}, \quad(x, z) \in \Gamma^{D}, \\
& h=h^{U}, \quad(x, z) \in \Gamma^{U}, \\
& \mathbf{q} \cdot \boldsymbol{\nu}=0, \quad(x, z) \in \Gamma^{L} \cup \Gamma^{R},
\end{aligned}
$$

siendo $\boldsymbol{\nu}$ el versor normal externo a la cara considerada.

La resolución del problema (3.1)-(3.5) permite obtener los campos $h(x, z)$ y $\mathbf{q}(x, z)$ en todo punto del dominio, y a partir de ellos es posible calcular el contenido de agua y la conductividad hidráulica de la muestra como se verá más adelante. Sin embargo, la resolución de este problema no es una tarea sencilla, puesto que la altura de presión interviene en forma no lineal en la ecuación (3.2). Para hallar la solución se procede a linealizar el problema mediante un método iterativo de Picard [21]. El esquema iterativo 
se define del siguiente modo:

$$
\begin{aligned}
& \boldsymbol{\nabla} \cdot \mathbf{q}^{i+1}=0, \quad(x, z) \in \Omega, \\
& \mathbf{q}^{i+1}=-K\left(h^{i}\right) \boldsymbol{\nabla}\left(h^{i+1}+z\right), \quad(x, z) \in \Omega, \\
& h^{i+1}=h^{D}, \quad(x, z) \in \Gamma^{D}, \\
& h^{i+1}=h^{U}, \quad(x, z) \in \Gamma^{U}, \\
& \mathbf{q}^{i+1} \cdot \boldsymbol{\nu}=0, \quad(x, z) \in \Gamma^{L} \cup \Gamma^{R},
\end{aligned}
$$

donde el supraíndice indica el nivel de iteración. Notar que para implementar el método es preciso establecer un valor inicial de iteración $h^{0}$. Luego, el problema (3.6)-(3.10) se resuelve en forma iterativa hasta alcanzar la convergencia, que se logra cuando la diferencia entre dos soluciones sucesivas es despreciable. En la sección siguiente se planteará una formulación mixta débil del problema (3.6)-(3.10).

\subsection{Formulación mixta débil}

Como paso previo a la implementación de un método mixto de elementos finitos se planteará la forma débil del problema (3.6)-(3.10). Para ello resulta necesario introducir la notación que será utilizada.

El producto interno en $L^{2}\left(\Omega_{j}\right)$ para funciones reales, y para cualquier subdominio $\Omega_{j} \subset \Omega$ se denota como:

$$
(v, w)_{\Omega_{j}}=\int_{\Omega_{j}} v w d \mathbf{x}
$$

donde $\mathbf{x}=(x, z)$, mientras que el producto interno en $L^{2}\left(\Gamma_{j}\right)$ para todo subconjunto $\Gamma_{j} \subset \Gamma$ se denota como:

$$
\langle v, w\rangle_{\Gamma_{j}}=\int_{\Gamma_{j}} v w d \sigma,
$$

siendo $d \sigma$ un diferencial de arco.

Los espacios de funciones que se utilizarán en la formulación mixta débil son los siguientes:

$$
\begin{gathered}
V=H(\operatorname{div}, \Omega)=\left\{\mathbf{v} \in\left[L^{2}(\Omega)\right]^{2}: \nabla . \mathbf{v} \in L^{2}(\Omega)\right\}, \\
V_{0}=\left\{\mathbf{v} \in V: \mathbf{v} \cdot \boldsymbol{\nu}=0, \quad(x, z) \in \Gamma^{L} \cup \Gamma^{R}\right\} \\
W=L^{2}(\Omega),
\end{gathered}
$$


donde el espacio de funciones vectoriales $V$ será utilizado para aproximar q y el espacio de funciones escalares $W$ para aproximar $h$.

Para obtener la forma débil se multiplican las ecuaciones (3.6) y (3.7) por las funciones de prueba $\psi \in W$ y $\mathbf{v} \in V_{0}$ respectivamente, para luego integrar las expresiones resultantes sobre el dominio completo $\Omega$. De esta manera, la formulación mixta débil del problema (3.6)-(3.10) se plantea del siguiente modo: Hallar $\left(h^{i+1}, \mathbf{q}^{i+1}\right) \in W \times V_{0}$ tal que:

$$
\begin{aligned}
& \left(\boldsymbol{\nabla} \cdot \mathbf{q}^{i+1}, \psi\right)_{\Omega}=0, \quad \psi \in W \\
& \left(\frac{\mathbf{q}^{i+1}}{K\left(h^{i}\right)}, \mathbf{v}\right)_{\Omega}-\left(h^{i+1}+z, \boldsymbol{\nabla} \cdot \mathbf{v}\right)_{\Omega} \\
& +\left\langle h^{D}, \mathbf{v} \cdot \boldsymbol{\nu}\right\rangle_{\Gamma^{D}}+\left\langle h^{U}+L_{z}, \mathbf{v} \cdot \boldsymbol{\nu}\right\rangle_{\Gamma^{U}}=0, \quad \mathbf{v} \in V_{0} .
\end{aligned}
$$

El paso siguiente para resolver el sistema (3.16)-(3.17) será definir un esquema de elementos finitos mixto.

\subsection{Método mixto de elementos finitos}

Consideremos una partición regular no solapada de $\Omega$ en subdominios rectangulares $\Omega_{j, k}$ tal que:

$$
\Omega=\bigcup_{j=1, k=1}^{N_{x}, N_{z}} \Omega_{j, k} \quad \text { con } \quad \Omega_{j, k}=\left[x_{j}, x_{j+1}\right] \times\left[z_{k}, z_{k+1}\right],
$$

donde $N_{x}$ y $N_{z}$ representan el número de subdominios en las direcciones $x$ y $z$ respectivamente. En la Fig. 3.2 (a) se ilustra un elemento genérico $\Omega_{j, k}$ de la partición, cuyo borde es $\Gamma_{j, k}=\Gamma_{j, k}^{1} \cup \Gamma_{j+1, k}^{1} \cup \Gamma_{j, k}^{2} \cup \Gamma_{j, k+1}^{2}$. Notar que se han utilizado los supraíndices 1 y 2 para diferenciar entre las caras normales al eje $x$ y las normales al eje $z$, respectivamente.

Para simplificar el sistema de ecuaciones que resulta de la aplicación convencional de un método mixto de elementos finitos, se suele realizar una formulación híbrida. La hibridización consiste en remover la condición de continuidad de la componente normal del flujo en los bordes interiores $\Gamma_{j, k}^{s}(s=1,2)$ de los subdominios. De este modo se introducen nuevos grados de libertad para el flujo, los cuales son indirectamente acoplados mediante un conjunto extra de ecuaciones que se definen utilizando un espacio de multiplicadores de Lagrange [4]. 


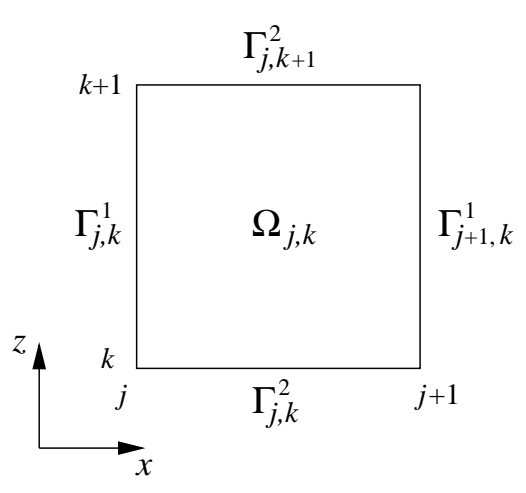

(a)

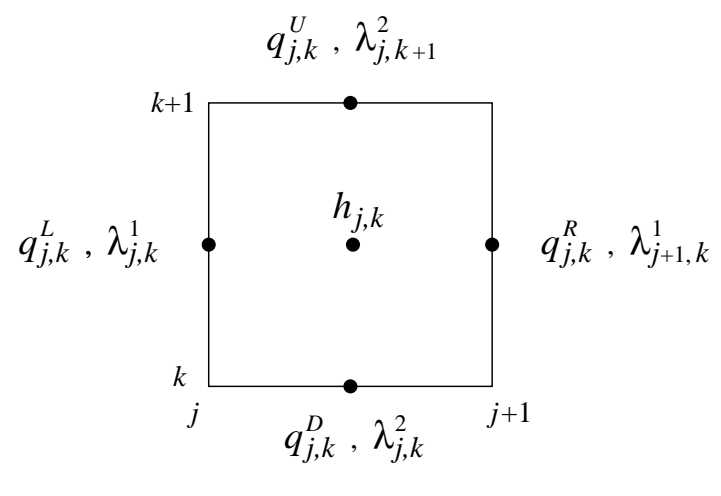

(b)

Figura 3.2: Elemento $\Omega_{j, k}$ de la partición de elementos finitos. En (a) se ilustra la nomenclatura utilizada para la frontera y en (b) se muestran las incógnitas del problema y los puntos a los que están asociadas.

Para realizar la formulación híbrida del problema definimos los siguientes espacios de elementos finitos de Raviart-Thomas-Nedelec de índice $m$ [83, 76]:

$$
\begin{gathered}
\Lambda^{m}=\left\{\lambda^{s}:\left.\lambda^{s}\right|_{\Gamma_{j, k}^{s}}=\lambda_{j, k}^{s} \in P_{m}\left(\Gamma_{j, k}^{s}\right), s=1,2\right\}, \\
V_{-1}^{m}=\left\{\mathbf{v} \in\left[L^{2}(\Omega)\right]^{2}:\left.\mathbf{v}\right|_{\Omega_{j, k}} \in R^{m}\right\}, \\
V_{0,-1}^{m}=\left\{\mathbf{v} \in V_{-1}^{m}: \mathbf{v} \cdot \boldsymbol{\nu}=0, \quad(x, z) \in \Gamma^{L} \cup \Gamma^{R}\right\}, \\
W^{m}=\left\{\psi \in L^{2}(\Omega):\left.\psi\right|_{\Omega_{j, k}} \in P_{m}\right\},
\end{gathered}
$$

donde $R^{m}=P_{m+1, m} \times P_{m, m+1}$ siendo $P_{l, m}$ polinomios definidos sobre $\Omega_{j, k}$ de grado no mayor que $l$ y $m$ en las variables $x$ y $z$, respectivamente. El espacio $\Lambda^{m}$ representa un espacio de multiplicadores de Lagrange cuyos elementos $\lambda_{j, k}^{s}$ están asociados al valor de altura hidráulica $\left(\lambda_{j, k}^{s} \sim h+z\right)$ en los bordes interiores $\Gamma_{j, k}^{s}$ (ver Fig. $3.2(\mathrm{~b})$ ).

El procedimiento mixto híbrido de elementos finitos se establece del siguiente modo: Dados $\left(h^{i}, \mathbf{q}^{i}, \lambda^{i}\right) \in W^{m} \times V_{0,-1}^{m} \times \Lambda^{m}$, hallar $\left(h^{i+1}, \mathbf{q}^{i+1}, \lambda^{i+1}\right) \in W^{m} \times V_{0,-1}^{m} \times \Lambda^{m}$ tal que:

$$
\sum_{j=1, k=1}^{N_{x}, N_{z}}\left(\nabla \cdot \mathbf{q}^{i+1}, \psi\right)_{\Omega_{j, k}}=0, \quad \psi \in W^{m}
$$


Capítulo 3. Diseño de experimentos numéricos 2D

$$
\begin{aligned}
& \sum_{j=1, k=1}^{N_{x}, N_{z}}\left(\frac{\mathbf{q}^{i+1}}{K\left(h^{i}\right)}, \mathbf{v}\right)_{\Omega_{j, k}}-\sum_{j=1, k=1}^{N_{x}, N_{z}}\left(h^{i+1}+z, \boldsymbol{\nabla} \cdot \mathbf{v}\right)_{\Omega_{j, k}} \\
& +\left\langle h^{D}, \mathbf{v} \cdot \boldsymbol{\nu}\right\rangle_{\Gamma^{D}}+\left\langle h^{U}+L_{z}, \mathbf{v} \cdot \boldsymbol{\nu}\right\rangle_{\Gamma^{U}} \\
& +\sum_{j=2, k=1}^{N_{x}, N_{z}}\left\langle\lambda_{j, k}^{1, i+1}, \mathbf{v} \cdot \boldsymbol{\nu}\right\rangle_{\Gamma_{j, k}^{1}}+\sum_{j=1, k=2}^{N_{x}, N_{z}}\left\langle\lambda_{j, k}^{2, i+1}, \mathbf{v} \cdot \boldsymbol{\nu}\right\rangle_{\Gamma_{j, k}^{2}}=0, \quad \mathbf{v} \in V_{0,-1}^{m}, \\
& \sum_{j=2, k=1}^{N_{x}, N_{z}}\left\langle\mu, \mathbf{q}^{i+1} \cdot \boldsymbol{\nu}\right\rangle_{\Gamma_{j, k}^{1}}+\sum_{j=1, k=2}^{N_{x}, N_{z}}\left\langle\mu, \mathbf{q}^{i+1} \cdot \boldsymbol{\nu}\right\rangle_{\Gamma_{j, k}^{2}}=0, \quad \mu \in \Lambda^{m} .
\end{aligned}
$$

Notar que la continuidad de las componentes normales del flujo a través de los bordes internos se ha impuesto mediante la ecuación (3.25).

\subsection{Implementación numérica}

Para la implementación numérica se utilizaron los espacios de Raviart-Thomas-Nedelec de orden cero $\left(\mathrm{RTN}_{0}\right)$. El empleo de estos espacios permite obtener un sistema algebraico con tantas incógnitas como número de elementos tenga la malla utilizada. Los espacios $\mathrm{RTN}_{0}$ bidimensionales son:

$$
\begin{gathered}
V_{-1}^{j, k}=\operatorname{span}\left\{\mathbf{v}_{j, k}^{L}, \mathbf{v}_{j, k}^{R}, \mathbf{v}_{j, k}^{D}, \mathbf{v}_{j, k}^{U}\right\} \\
W^{j, k}=\operatorname{span}\left\{\psi_{j, k}\right\}
\end{gathered}
$$

donde

$$
\begin{aligned}
& \mathbf{v}_{j, k}^{L}(x, z)= \begin{cases}\left(-1+\frac{x-x_{j}}{\Delta x_{j}}, 0\right) & (x, z) \in \Omega_{j, k} \\
0 & (x, z) \notin \Omega_{j, k},\end{cases} \\
& \mathbf{v}_{j, k}^{R}(x, z)= \begin{cases}\left(\frac{x-x_{j}}{\Delta x_{j}}, 0\right) & (x, z) \in \Omega_{j, k} \\
0 & (x, z) \notin \Omega_{j, k},\end{cases} \\
& \mathbf{v}_{j, k}^{D}(x, z)= \begin{cases}\left(0,-1+\frac{z-z_{k}}{\Delta z_{k}}\right) & (x, z) \in \Omega_{j, k} \\
0 & (x, z) \notin \Omega_{j, k},\end{cases} \\
& \mathbf{v}_{j, k}^{U}(x, z)= \begin{cases}\left(0, \frac{z-z_{k}}{\Delta z_{k}}\right) & (x, z) \in \Omega_{j, k} \\
0 & (x, z) \notin \Omega_{j, k},\end{cases} \\
& \psi_{j, k}(x, z)= \begin{cases}1 & (x, z) \in \Omega_{j, k} \\
0 & \end{cases}
\end{aligned}
$$


siendo $\Delta x_{j}=x_{j+1}-x_{j}$ y $\Delta z_{k}=z_{k+1}-z_{k}$.

La solución de (3.23)-(3.25) en cualquier punto del dominio se expresa como:

$$
\begin{gathered}
h^{i+1}(x, z)=\sum_{j=1, k=1}^{N_{x}, N_{z}} h_{j, k}^{i+1} \psi_{j, k}(x, z), \\
\mathbf{q}^{i+1}(x, z)=\sum_{j=1, k=1}^{N_{x}, N_{z}}\left[q_{j, k}^{L, i+1} \mathbf{v}_{j, k}^{L}(x, z)+q_{j, k}^{R, i+1} \mathbf{v}_{j, k}^{R}(x, z)\right. \\
\left.+q_{j, k}^{D, i+1} \mathbf{v}_{j, k}^{D}(x, z)+q_{j, k}^{U, i+1} \mathbf{v}_{j, k}^{U}(x, z)\right],
\end{gathered}
$$

donde $h_{j, k}^{i+1}$ se asocia al punto medio del elemento $\Omega_{j, k}$ y los flujos normales $q_{j, k}^{S, i+1}(S=$ $L, R, D, U)$ al punto medio de la cara respectiva señalada por su supraíndice. Como ya se ha mencionado, los valores de $\lambda_{j, k}^{s}$ están asociados a los de $h+z$ sobre los bordes internos del elemento (ver Fig. $3.2(\mathrm{~b})$ ).

Seleccionando $\mathbf{v}=\mathbf{v}^{L}, \mathbf{v}^{R}, \mathbf{v}^{D}$ y $\mathbf{v}^{U}$ en (3.24) y utilizando una regla trapezoidal para calcular el primer término de esta ecuación, se obtienen las siguientes expresiones para $q_{j, k}^{L, i+1}, q_{j, k}^{R, i+1}, q_{j, k}^{D, i+1}$ y $q_{j, k}^{U, i+1}$ :

$$
\begin{aligned}
& q_{j, k}^{L, i+1}= \begin{cases}\frac{2 K_{j, k}^{L, i}}{\Delta x_{j}}\left[h_{j, k}^{i+1}+\frac{1}{2}\left(z_{k}+z_{k+1}\right)-\lambda_{j, k}^{1, i+1}\right] & j=2, \ldots, N_{x} \\
0 & j=1,\end{cases} \\
& q_{j, k}^{R, i+1}= \begin{cases}\frac{2 K_{j, k}^{R, i}}{\Delta x_{j}}\left[h_{j, k}^{i+1}+\frac{1}{2}\left(z_{k}+z_{k+1}\right)-\lambda_{j+1, k}^{1, i+1}\right] & j=1, \ldots, N_{x}-1 \\
0 & j=N_{x},\end{cases} \\
& q_{j, k}^{D, i+1}= \begin{cases}\frac{2 K_{j, k}^{D, i}}{\Delta z_{k}}\left[h_{j, k}^{i+1}+\frac{1}{2}\left(z_{k}+z_{k+1}\right)-\lambda_{j, k}^{2, i+1}\right] & k=2, \ldots, N_{z} \\
\frac{2 K_{j, k}^{D, i}}{\Delta z_{k}}\left[h_{j, k}^{i+1}+\frac{1}{2}\left(z_{k}+z_{k+1}\right)-h^{D}-z_{k}\right] & k=1,\end{cases} \\
& q_{j, k}^{U, i+1}= \begin{cases}\frac{2 K_{j, k}^{U, i}}{\Delta z_{k}}\left[h_{j, k}^{i+1}+\frac{1}{2}\left(z_{k}+z_{k+1}\right)-\lambda_{j, k+1}^{2, i+1}\right] & k=1, \ldots, N_{z}-1 \\
\frac{2 K_{j, k}^{U, i}}{\Delta z_{k}}\left[h_{j, k}^{i+1}+\frac{1}{2}\left(z_{k}+z_{k+1}\right)-h^{U}-z_{k+1}\right] & k=N_{z},\end{cases}
\end{aligned}
$$

siendo

$$
\begin{aligned}
& K_{j, k}^{L, i}=K\left(\lambda_{j, k}^{1, i}-\frac{\left(z_{k}+z_{k+1}\right)}{2}\right), \quad K_{j, k}^{R, i}=K\left(\lambda_{j+1, k}^{1, i}-\frac{\left(z_{k}+z_{k+1}\right)}{2}\right), \\
& K_{j, k}^{D, i}=K\left(\lambda_{j, k}^{2, i}-z_{k}\right), \quad K_{j, k}^{U, i}=K\left(\lambda_{j, k+1}^{2, i}-z_{k+1}\right) .
\end{aligned}
$$

Notar que para evaluar $K(h)$ en los bordes de los elementos se han utilizado los multiplicadores de Lagrange. 
La ecuación (3.25) equivale a:

$$
\begin{array}{ll}
q_{j, k}^{L, i+1}+q_{j-1, k}^{R, i+1}=0, & j=2, \ldots, N_{x}, \quad k=1, \ldots, N_{z}, \\
q_{j, k}^{D, i+1}+q_{j, k-1}^{U, i+1}=0, & j=1, \ldots, N_{x}, \quad k=2, \ldots, N_{z} .
\end{array}
$$

Reemplazando (3.35)-(3.38) en (3.40)-(3.41) se obtienen las siguientes expresiones para los multiplicadores de Lagrange $\lambda_{j, k}^{s, i+1}$ en términos de la altura de presión $h_{j, k}^{i+1}$ :

$$
\begin{aligned}
\lambda_{j, k}^{1, i+1} & =\frac{K_{j, k}^{L, i} \Delta x_{j-1}}{\Delta x_{j-1} K_{j, k}^{L, i}+\Delta x_{j} K_{j-1, k}^{R, i}} h_{j, k}^{i+1} \\
& +\frac{K_{j-1, k}^{R, i} \Delta x_{j}}{\Delta x_{j-1} K_{j, k}^{L, i}+\Delta x_{j} K_{j-1, k}^{R, i}} h_{j-1, k}^{i+1} \\
& +\frac{1}{2}\left(z_{k+1}+z_{k}\right), \quad j=2, \ldots, N_{x}, \quad k=1, \ldots, N_{z}, \\
\lambda_{j, k}^{2, i+1}= & \frac{K_{j, k}^{D, i} \Delta z_{k-1}}{\Delta z_{k-1} K_{j, k}^{D, i}+\Delta z_{k} K_{j, k-1}^{U, i}}\left[h_{j, k}^{i+1}+\frac{1}{2}\left(z_{k}+z_{k+1}\right)\right] \\
+ & \frac{K_{j, k-1}^{U, i} \Delta z_{k}}{\Delta z_{k-1} K_{j, k}^{D, i}+\Delta z_{k} K_{j, k-1}^{U, i}}\left[h_{j, k-1}^{i+1}+\frac{1}{2}\left(z_{k-1}+z_{k}\right)\right], \\
& j=1, \ldots, N_{x}, \quad k=2, \ldots, N_{z} .
\end{aligned}
$$

Por otra parte, tomando $\psi_{j, k}(x, z)$ en (3.23) resulta:

$$
\frac{q_{j, k}^{L, i+1}+q_{j, k}^{R, i+1}}{\Delta x_{j}}+\frac{q_{j, k}^{D, i+1}+q_{j, k}^{U, i+1}}{\Delta z_{k}}=0 .
$$

Finalmente, reemplazando (3.35)-(3.38) en (3.44), y haciendo uso de (3.42) y (3.43), se obtiene el siguiente sistema de ecuaciones:

$$
L_{j, k}^{i} h_{j-1, k}^{i+1}+D_{j, k}^{i} h_{j, k-1}^{i+1}+C_{j, k}^{i} h_{j, k}^{i+1}+R_{j, k}^{i} h_{j+1, k}^{i+1}+U_{j, k}^{i} h_{j, k+1}^{i+1}=R H S_{j, k}^{i},
$$

siendo

$$
\begin{array}{ll}
L_{j, k}^{i}=-\left[1-\delta_{1, j}\right] \frac{A_{j, k}^{L, i}}{\Delta x_{j}}, & R_{j, k}^{i}=-\left[1-\delta_{N_{x}, j}\right] \frac{A_{j, k}^{R, i}}{\Delta x_{j}}, \\
D_{j, k}^{i}=-\left[1-\delta_{1, k}\right] \frac{A_{j, k}^{D, i}}{\Delta z_{k}}, & U_{j, k}^{i}=-\left[1-\delta_{N_{z}, k}\right] \frac{A_{j, k}^{U, i}}{\Delta z_{k}},
\end{array}
$$


Capítulo 3. Diseño de experimentos numéricos 2D

$$
\begin{gathered}
C_{j, k}^{i}=\left[1-\delta_{1, j}\right] \frac{A_{j, k}^{L, i}}{\Delta x_{j}}+\left[1-\delta_{1, k}\right] \frac{A_{j, k}^{D, i}}{\Delta z_{k}} \\
+\left[1-\delta_{N_{x}, j}\right] \frac{A_{j, k}^{R, i}}{\Delta x_{j}}+\left[1-\delta_{N_{z}, k}\right] \frac{A_{j, k}^{U, i}}{\Delta z_{k}} \\
+\delta_{1, k} \frac{2 K_{j, k}^{D, i}}{\Delta z_{k}^{2}}+\delta_{N_{z}, k} \frac{2 K_{j, k}^{U, i}}{\Delta z_{k}^{2}}, \\
R H S_{j, k}^{i}=\delta_{1, k} \frac{2 K_{j, k}^{D, i}\left[h^{D}-\Delta z_{k}\right]}{\Delta z_{k}^{2}}+\delta_{N_{z}, k} \frac{2 K_{j, k}^{U, i}\left[h^{U}+\Delta z_{k}\right]}{\Delta z_{k}^{2}} \\
-\left[1-\delta_{1, k}\right] \frac{A_{j, k}^{D, i}}{\Delta z_{k}} \frac{\left[z_{k+1}-z_{k-1}\right]}{2}+\left[1-\delta_{N_{z}, k}\right] \frac{A_{j, k}^{U, i}}{\Delta z_{k}} \frac{\left[z_{k+2}-z_{k}\right]}{2}
\end{gathered}
$$

donde

$$
\begin{aligned}
& A_{j, k}^{L, i}=\frac{2 K_{j, k}^{L, i} K_{j-1, k}^{R, i}}{\Delta x_{j-1} K_{j, k}^{L, i}+\Delta x_{j} K_{j-1, k}^{R, i}}, \quad A_{j, k}^{R, i}=\frac{2 K_{j+1, k}^{L, i} K_{j, k}^{R, i}}{\Delta x_{j} K_{j+1, k}^{L, i}+\Delta x_{j+1} K_{j, k}^{R, i}}, \\
& A_{j, k}^{D, i}=\frac{2 K_{j, k}^{D, i} K_{j, k-1}^{U, i}}{\Delta z_{k-1} K_{j, k}^{D, i}+\Delta z_{k} K_{j, k-1}^{U, i}}, \quad A_{j, k}^{U, i}=\frac{2 K_{j, k+1}^{D, i} K_{j, k}^{U, i}}{\Delta z_{k} K_{j, k+1}^{D, i}+\Delta z_{k+1} K_{j, k}^{U, i}},
\end{aligned}
$$

y $\delta_{j, k}$ es la Delta de Kronecker, definida como:

$$
\delta_{j, k}= \begin{cases}1, & j=k \\ 0, & j \neq k .\end{cases}
$$

\subsection{Esquema general de la simulación}

El código computacional para resolver el sistema de ecuaciones resultante fue desarrollado en lenguaje Fortran. La estructura del algoritmo puede resumirse en los siguientes pasos:

1. Se asigna un valor inicial para el proceso iterativo de Picard considerando la siguiente expresión para $h^{0}(x, z)$ :

$$
h^{0}(x, z)=\left(\frac{h^{U}-h^{D}}{L_{z}}\right) z+h^{D} .
$$

2. Se resuelve el sistema (3.45) y se obtiene $h_{j, k}^{i+1}$. 
3. Se evalúa el módulo de la diferencia $\delta h_{j, k}^{i+1}=h_{j, k}^{i+1}-h_{j, k}^{i}$ sobre todo el dominio. Si la norma de $\delta h_{j, k}^{i+1}$ es menor que cierta tolerancia prefijada, la iteración concluye y $h_{j, k}^{i+1}$ es la solución numérica deseada. De lo contrario, se vuelve al paso 2 definiendo $h_{j, k}^{i}=h_{j, k}^{i+1}$.

4. Se calculan los valores del campo de flujo $q_{j, k}^{S, i+1}(S=L, R, D, U)$ utilizando (3.35)$(3.38)$.

El sistema de ecuaciones del paso 2 se resuelve utilizando un método de sobrerrelajación por líneas (Método LSOR [79]).

\subsection{Cálculo de las relaciones constitutivas}

El método numérico que se ha presentado permite obtener en forma aproximada el campo de altura de presión $h$ y el flujo q en cada elemento $\Omega_{j, k}$ de la partición. Aunque estos campos suelen en general registrar variaciones importantes dentro de la muestra, se asume que el valor efectivo de altura de presión $h^{e f}$ (es decir el valor de $h$ representativo de la muestra) viene dado por la ecuación (2.33) [51, 59]. Como el aire se asume a presión atmosférica, esta ecuación se escribe simplemente como:

$$
h^{e f}=\frac{h^{D}+h^{U}}{2} .
$$

Vale aclarar que el significado del término "efectivo" en este contexto no es el mismo que el utilizado para definir la saturación efectiva o el contenido efectivo de agua. Con el objeto de resaltar esta distinción y evitar confusión, se utilizará el supraíndice "ef" para denotar cualquier cantidad efectiva de la muestra.

De acuerdo con la definición del contenido de agua (2.12), este podrá calcularse como el cociente entre el volumen total de agua y el volumen de la muestra:

$$
\theta^{e f}=\frac{\int_{\Omega} \theta(h) d V}{\int_{\Omega} d V}
$$

donde $\theta(h)$ denota el contenido de agua en cada punto del dominio. En el caso bidimensional y discreto, la fórmula (3.53) se puede aproximar por:

$$
\theta^{e f}=\frac{1}{L_{x} L_{z}} \sum_{j=1, k=1}^{N_{x}, N_{z}} \theta\left(h_{j, k}\right) \Delta x_{j} \Delta z_{k}
$$


Los contenidos de agua residual y máximo de la muestra pueden calcularse análogamente a $\theta^{e f}$, resultando para sus versiones discretas:

$$
\begin{aligned}
& \theta_{r}^{e f}=\frac{1}{L_{x} L_{z}} \sum_{j=1, k=1}^{N_{x}, N_{z}} \theta_{r, j, k} \Delta x_{j} \Delta z_{k} \\
& \theta_{s}^{e f}=\frac{1}{L_{x} L_{z}} \sum_{j=1, k=1}^{N_{x}, N_{z}} \theta_{s, j, k} \Delta x_{j} \Delta z_{k}
\end{aligned}
$$

siendo $\theta_{r, j, k}$ y $\theta_{s, j, k}$ los contenidos de agua residual y máximo en el elemento $\Omega_{j, k}$. Luego, la saturación efectiva de la muestra $S_{e}^{e f}$ puede calcularse de la siguiente manera:

$$
S_{e}^{e f}=\frac{\theta^{e f}-\theta_{r}^{e f}}{\theta_{s}^{e f}-\theta_{r}^{e f}}=\frac{\sum_{j=1, k=1}^{N_{x}, N_{z}}\left[\theta\left(h_{j, k}\right)-\theta_{r, j, k}\right] \Delta x_{j} \Delta z_{k}}{\sum_{j=1, k=1}^{N_{x}, N_{z}}\left[\theta_{s, j, k}-\theta_{r, j, k}\right] \Delta x_{j} \Delta z_{k}} .
$$

Para obtener la conductividad hidráulica efectiva $K^{e f}$ haremos uso de la ecuación (2.32), para lo cual es necesario calcular el flujo neto a través de la muestra:

$$
q^{e f}=\frac{\int_{A} \mathbf{q} \cdot \boldsymbol{\nu} d A}{A}
$$

donde $A$ es una sección cualquiera transversal a la dirección del flujo. Notar que al no existir fuentes ni sumideros y al ser el flujo estacionario, la divergencia de q resulta nula (ver ecuación (3.1)). Luego, el flujo total a través de la muestra es independiente de la sección $A$ que se considere. Por simplicidad tomaremos la cara $\Gamma^{D}$, resultando para la forma discreta de $q^{e f}$ :

$$
q^{e f}=\frac{\sum_{j=1}^{N_{x}} q_{j, 1}^{D} \Delta x_{j}}{L_{x}} .
$$

Por otra parte, aplicando la ley de Darcy a escala de la muestra se tiene que:

$$
q^{e f}=K^{e f}\left(h^{e f}\right)\left(\frac{h^{U}-h^{D}}{L_{z}}+1\right) .
$$

Luego, combinando (3.59) y (3.60) se obtiene la siguiente expresión para la conductividad hidráulica efectiva:

$$
K^{e f}\left(h^{e f}\right)=\frac{q^{e f}}{\left(\frac{h^{U}-h^{D}}{L_{z}}+1\right)}=\frac{\sum_{j=1}^{N_{x}} q_{j, 1}^{D} \Delta x_{j}}{L_{x}\left(\frac{h^{U}-h^{D}}{L_{z}}+1\right)} .
$$

Para obtener la conductividad hidráulica saturada de la muestra basta con efectuar el experimento para una altura de presión efectiva positiva, lo cual puede lograrse imponiendo valores positivos para $h^{D}$ y $h^{U}$. En este caso el problema diferencial (3.1)-(3.5) 
es lineal y puede resolverse en forma directa sin necesidad de implementar un método iterativo. Luego, la conductividad hidráulica saturada efectiva puede obtenerse como:

$$
K_{s}^{e f}=\frac{q^{e f, s a t}}{\frac{h^{U}-h^{D}}{L_{z}}+1} .
$$

donde $q^{e f, s a t}$ es el flujo efectivo saturado.

La permeabilidad efectiva $\kappa^{e f}$ puede calcularse a partir de (3.62) haciendo uso de la ecuación (2.26):

$$
\kappa^{e f}=\frac{\mu K_{s}^{e f}}{\rho g} .
$$

Resta definir la conductividad hidráulica relativa efectiva $K_{r}^{e f}$, la cual se obtiene a partir de la definición de $K$ dada por la ecuación (2.25):

$$
K_{r}^{e f}=\frac{K^{e f}}{K_{s}^{e f}},
$$

completando de esta manera el cálculo de las relaciones constitutivas efectivas para la altura de presión $h^{e f}$.

De forma análoga a la determinación experimental, las curvas de $\theta^{e f}\left(h^{e f}\right)$ y $K^{e f}\left(h^{e f}\right)$ (o $S_{e}^{e f}\left(h^{e f}\right)$ y $\left.K_{r}^{e f}\left(h^{e f}\right)\right)$ se obtienen repitiendo el experimento numérico para distintos valores de $h^{e f}$, lo cual se logra cambiando las condiciones de borde $h^{D}$ y $h^{U}$. Nótese sin embargo que para un dado valor de $h^{e f}$ existen infinitos valores de $h^{D}$ y $h^{U}$ que satisfacen la ecuación (3.52). A efectos de simplificar los cálculos consideraremos:

$$
h^{U}=\frac{5}{4} h^{e f} \text { y } h^{D}=\frac{3}{4} h^{e f},
$$

De este modo el gradiente hidráulico aplicado a la muestra será:

$$
\frac{h^{U}-h^{D}}{L_{z}}+1=\frac{1}{2 L_{z}} h^{e f}+1 .
$$

Notar que con las condiciones (3.65) el gradiente de altura de presión $\left(h^{U}-h^{D}\right) / L_{z}$ es proporcional al valor de $h^{e f}$. Esto permite obtener una descripción más realista del experimento numérico, dado que en las pruebas de laboratorio, cuanto menor es la saturación (y por tanto, mayor es $\left|h^{e f}\right|$ ) mayor es el gradiente hidráulico necesario para que se desarrolle el flujo.

Los cálculos que se han presentado en esta sección han sido implementados mediante el diseño de subrutinas Fortran acopladas al código de simulación de flujo. A continuación 
se ilustrará el método propuesto mediante dos ejemplos que involucran el cálculo de relaciones constitutivas para rocas fracturadas y el cálculo de la permeabilidad efectiva para el análisis de la atenuación y dispersión de ondas sísmicas en rocas heterogéneas.

\subsection{Relaciones constitutivas para rocas fracturadas}

Para poder calcular las relaciones constitutivas comenzaremos por generar en forma sintética una muestra de roca fracturada. El patrón de la red de fracturas se definirá sobre una malla de elementos rectangulares. Los elementos serán lo suficientemente pequeños como para asegurar que las fracturas más angostas sean representables. Consideraremos un medio con fracturas verticales y horizontales que se cruzan perpendicularmente en un punto arbitrario del dominio [59].

Para generar la red de fracturas debe establecerse en primer lugar el número total de fracturas que contendrá el dominio. La mitad de este número es la cantidad de centros de fractura que contendrá la muestra, dado que en torno de cada centro se desarrollarán dos fracturas, una horizontal y otra vertical, ambas centradas en dicho punto. Luego se generan aleatoriamente los puntos en donde estos centros estarán situados en el dominio. Cada una de las fracturas tendrá propiedades geométricas características, es decir, apertura y longitud. Vale aclarar que estamos considerando un modelo simplificado para representar una fractura, puesto que en general las paredes no son planas sino rugosas. Por otra parte debe entenderse que, dado que en realidad las paredes de las fracturas son irregulares dentro de ciertos límites, la apertura que utilizaremos para cada fractura en este modelo simplificado representa una apertura media de la misma. Siguiendo las ideas del trabajo de Liu y Bodvarsson [59] utilizaremos una ley fractal que relaciona la longitud de la fractura con la apertura de la misma::

$$
b=c L^{d}
$$

siendo $b$ la apertura, $L$ la longitud, $d$ la dimensión fractal y $c$ una constante. Una vez determinados los parámetros que caracterizan a la fractura, la misma queda definida geométricamente y solo resta asignar los parámetros hidráulicos correspondientes a cada elemento de la malla que se encuentre dentro del subdominio de la fractura. Los elementos restantes tendrán las propiedades hidráulicas de la matriz rocosa.

En la Fig. 3.3 se han representado dos dominios de $10 \mathrm{~cm}$ de lado, discretizados mediante una partición regular de $100 \times 100$ elementos. Ambos medios fueron generados 
(a)

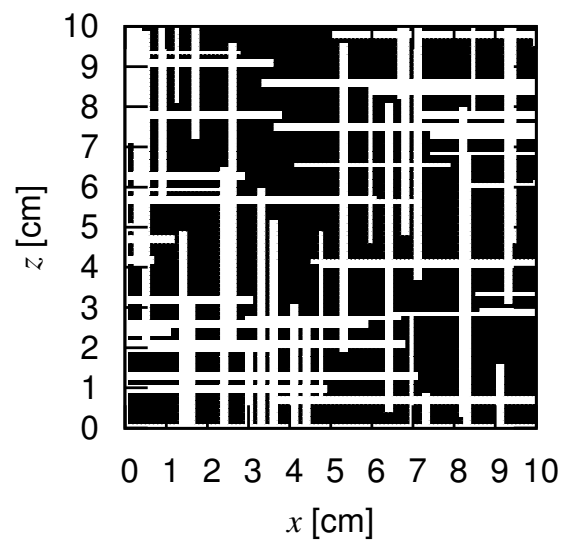

(b)

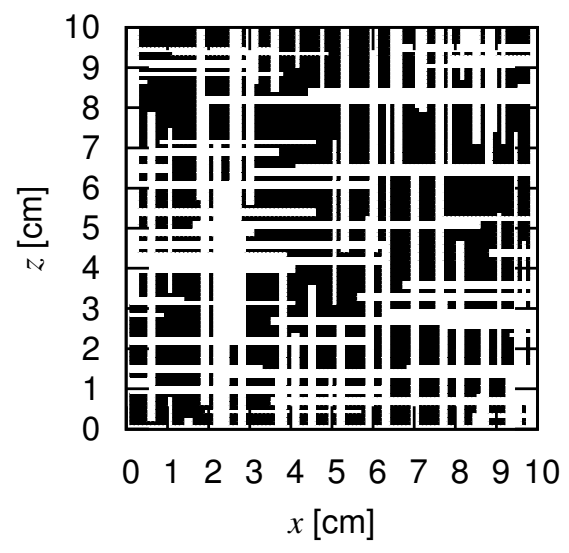

Figura 3.3: Muestras sintéticas de roca fracturada.

mediante el método que hemos descrito. Los subdominios correspondientes a las fracturas son blancos y el correspondiente a la matriz negro. En ambos ejemplos se han utilizado dos aperturas diferentes, $b_{1}=0.1 \mathrm{~cm} \mathrm{y} b_{2}=0.2 \mathrm{~cm}$. Los números totales de fracturas en cada ejemplo son 90 (a) y 180 (b), respectivamente. Los valores de los parámetros de la ley fractal (3.67) que se han considerado para estos ejemplos son $c=0.02$ y $d=1.13$.

Para la caracterización hidráulica de la matriz de la roca se han utilizado los parámetros de van Genuchten calibrados por Unger et al. [107] para los basaltos de Box Canyon Site (Idaho, EEUU). Se asume también que las propiedades hidráulicas de las fracturas pueden ser descritas por lo parámetros de van Genuchten obtenidos por Carsel y Parrish [19] para un medio cuya textura corresponde a una arena margosa. Los parámetros de van Genuchten para la matriz y las fracturas se listan en la Tabla (3.1). Notar que la conductividad hidráulica de las fracturas es 3 órdenes de magnitud mayor que la correspondiente a la matriz.

Para verificar que el tamaño de las muestras constituye en cada caso un REV de roca fracturada, se efectuó el cálculo de la porosidad en función del tamaño de la muestra como fue descrito en el Capítulo 2. La porosidad de cada subdominio de la muestra puede calcularse utilizando la fórmula (3.56) asumiendo que la saturación máxima es unitaria. Los resultados de este cálculo se muestran en la Fig. 3.4. Como puede observarse, la porosidad tiende a estabilizarse cuando el tamaño del subdominio considerado es mayor 


\begin{tabular}{|c|c|c|c|c|c|}
\hline Textura & $\theta_{s}$ & $\theta_{r}$ & $\alpha_{v g}\left[\mathrm{~cm}^{-1}\right]$ & $n$ & $K_{s}[\mathrm{~cm} / \mathrm{d}]$ \\
\hline Fracturas & 0.41 & 0.057 & 0.124 & 2.28 & 350.2 \\
\hline Matriz & 0.2 & 0.1 & 0.049 & 1.33 & 0.281 \\
\hline
\end{tabular}

Tabla 3.1: Parámetros de van Genuchten para las fracturas y la matriz.

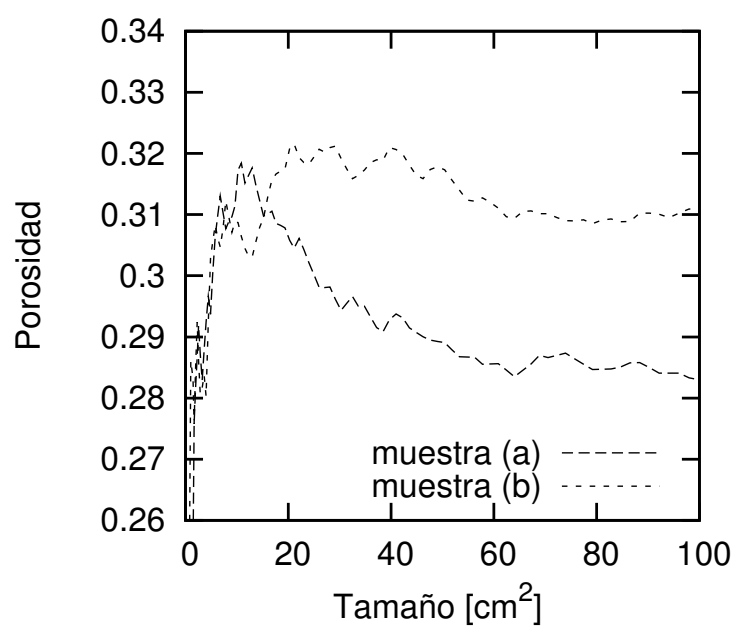

Figura 3.4: Porosidad en función del tamaño de la muestra.

que $60 \mathrm{~cm}^{2}$ aproximadamente, lo cual garantiza la representatividad de las muestras.

Las relaciones constitutivas fueron obtenidas considerado 6 valores de $h^{\text {ef }}$ por década logarítmica en el rango de $-1 \mathrm{~cm} \mathrm{a}-10^{3} \mathrm{~cm}$. La conductividad hidráulica saturada se calculó considerando $h^{e f}=10 \mathrm{~cm}$, obteniéndose $K_{s}^{e f}=68.75 \mathrm{~cm} / \mathrm{d}$ para la muestra (a) y $K_{s}^{e f}=104.91 \mathrm{~cm} / \mathrm{d}$ para la muestra (b). Luego, se contabiliza un total de 19 simulaciones sobre cada muestra (una para cada valor de $h^{e f}$ ). La Fig. 3.5 muestra los resultados para $S_{e}^{e f}$ en función del valor absoluto de $h^{e f}$ (izquierda) y para $K_{r}^{e f}$ en función de $S_{e}^{e f}$ (derecha). Las figura muestra además las curvas correspondientes al modelo constitutivo de van Genuchten para las texturas de las fracturas y de la matriz.

Puede observarse que para valores pequeños de $\left|h^{e f}\right|$, cercanos a la saturación máxima, las muestras tienden a comportarse según la textura de las fracturas. A medida que aumenta la altura de presión (en valor absoluto) los valores simulados se aproximan a la 

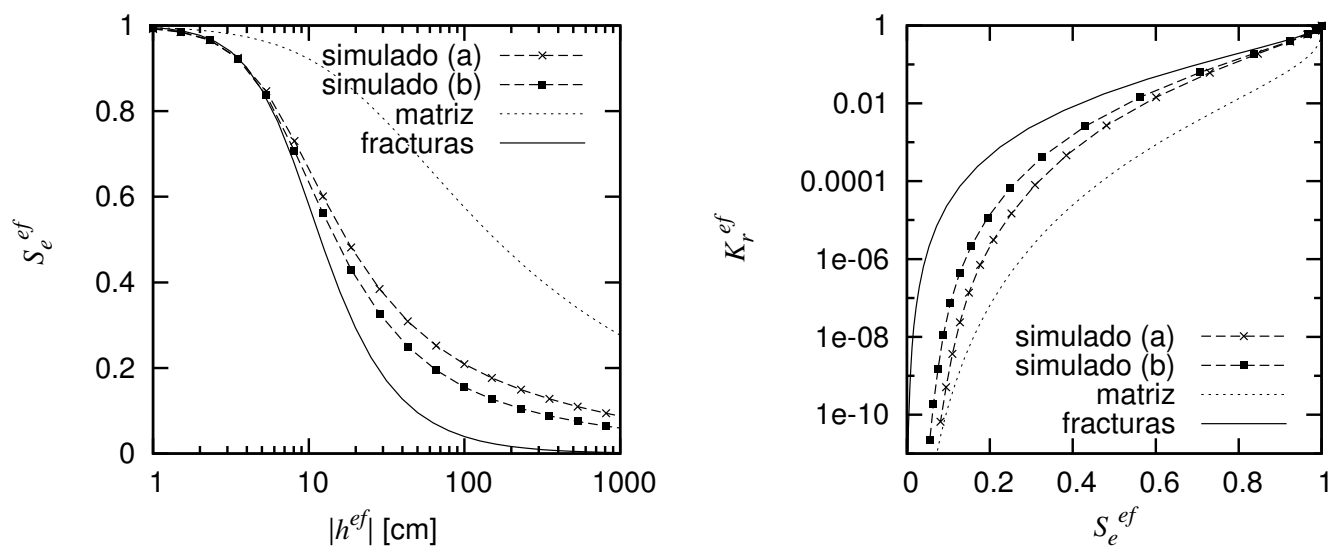

Figura 3.5: Curvas de $S_{e}^{e f}\left(h^{e f}\right)$ (izquierda) y $K_{r}^{e f}\left(S_{e}^{e f}\right)$ (derecha). Resultado de la simulación para las muestras (a) y (b) y curvas de van Genuchten para las texturas de la matriz y las fracturas.

curva de la matriz, mostrando claramente que en estas condiciones las fracturas no juegan un papel importante (contrariamente a lo que ocurre cerca de la saturación máxima). Para estos valores, el agua presente en las fracturas ha drenado casi por completo debido a su mayor conductividad. Notar además que las curvas correspondientes a la muestra (b), cuyo contenido de fracturas es mayor que el de la muestra (a), se aproxima más al comportamiento de la la curva de van Genuchten correspondiente a las fracturas.

Finalmente, y a modo de validación del procedimiento numérico, se consideró una muestra de roca homogénea. En este caso las relaciones constitutivas simuladas deberían coincidir con las expresiones analíticas del modelo de van Genuchten. En la Fig. 3.6 se muestran los resultados para muestras homogéneas correspondientes a las texturas de la matriz y las fracturas. En ambos casos las relaciones obtenidas en forma numérica coinciden con las expresiones analíticas, lo que no solo constituye una validación del experimento numérico, sino también del modelo conceptual propuesto.

\subsection{Permeabilidad efectiva en rocas con heterogeneidades fractales}

En la presente sección se mostrará a partir de un análisis comparativo que la técnica numérica propuesta constituye una herramienta de gran utilidad en el estudio de las propiedades sísmicas de medios porosos altamente heterogéneos. Recientemente se ha 

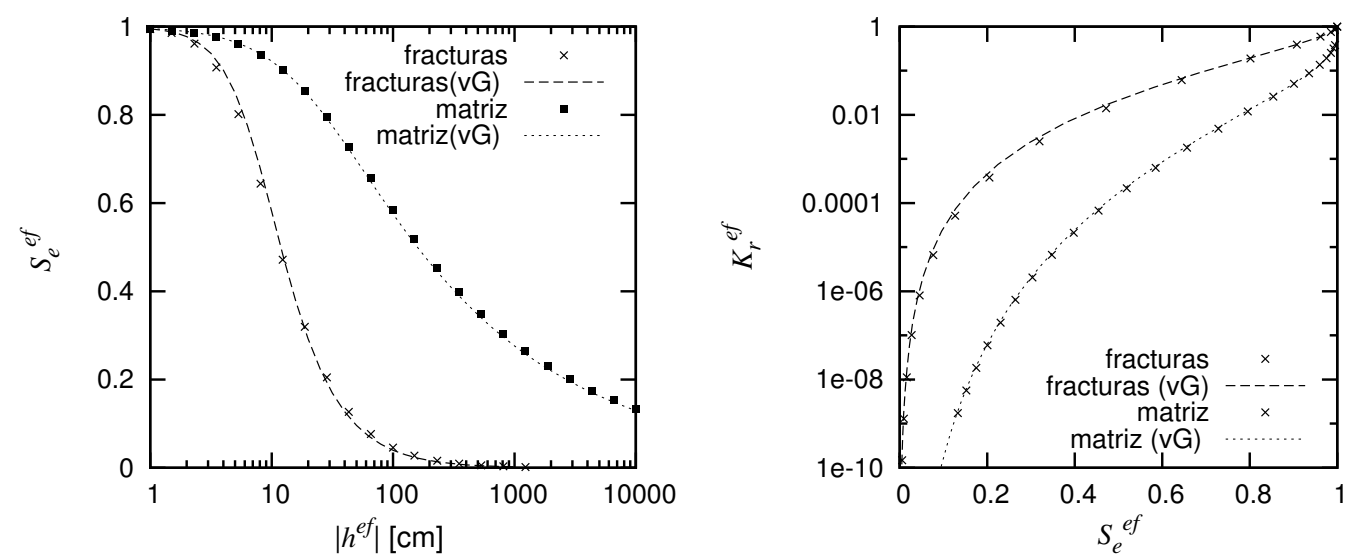

Figura 3.6: Curvas de $S_{e}^{e f}\left(h^{e f}\right)$ (izquierda) y $K_{r}^{e f}\left(S_{e}^{e f}\right)$ (derecha). Resultado de la simulación considerando un medio homogéneo con la textura de las fracturas y con la textura de la matriz. Los puntos corresponden a los valores simulados y las líneas de trazo a los modelos de van Genuchten (vG) para ambas texturas.

demostrado que la permeabilidad de medios porosos heterogéneos está estrechamente vinculada con los mecanismos de atenuación y dispersión de ondas sísmicas. Cuando una onda sísmica se propaga a través de un medio poroso saturado que presenta heterogeneidades mesoscópicas (esto es, heterogeneidades de tamaño mayor que la escala poral pero menores que la longitud de onda sísmica) se establecen gradientes de presión locales que inducen el flujo a través de los límites de las heterogeneidades. Este mecanismo es el denominado Flujo Inducido por Ondas (WIFF') y es uno de los mecanismos más importantes en el estudio de la atenuación y dispersión de ondas sísmicas en medios porosos con heterogeneidades mesoscópicas [75]. La existencia de este mecanismo establece un vínculo entre la respuesta sísmica y la permeabilidad del medio, lo que ha llevado a la definición de un nuevo concepto de permeabilidad. Se define la permeabilidad sísmica como la permeabilidad efectiva (es decir, representativa del medio heterogéneo) que logra predecir la atenuación y dispersión sufrida por las ondas sísmicas en dicho medio.

Desafortunadamente no existe en la actualidad un método que permita estimar la permeabilidad sísmica para este tipo de medios. Una alternativa viable consiste en reemplazar el campo de permeabilidad por un campo homogéneo equivalente que logre predecir las mismas curvas de atenuación y dispersión observadas para el medio heterogéneo.

\footnotetext{
${ }^{2}$ del inglés Wave Induced Fluid Flow.
} 


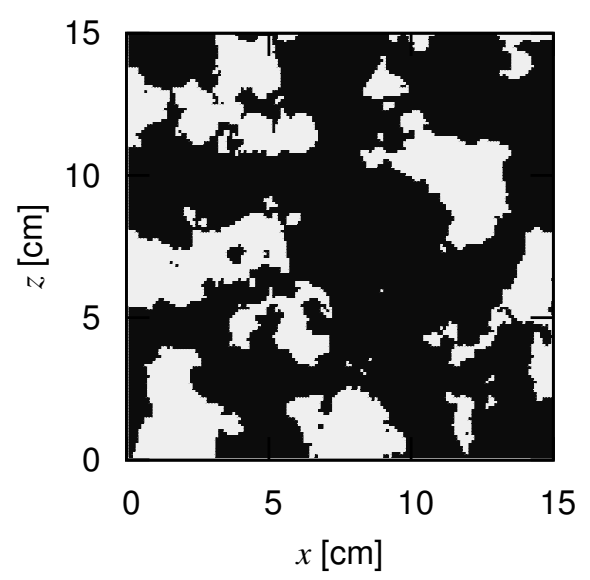

Figura 3.7: Muestra de roca con heterogeneidades de tipo fractal.

Dado que la permeabilidad hidráulica efectiva es una cantidad representativa del flujo que puede desarrollarse en una muestra, es razonable suponer que la misma esté vinculada con la permeabilidad sísmica, y es por ello que su determinación resulta de interés en este tipo de estudios.

El análisis que se presenta en esta sección consiste en comparar las curvas de atenuación y dispersión de una muestra de roca heterogénea con las curvas que se obtienen al reemplazar el campo de permeabilidad heterogéneo por un campo homogéneo equivalente. Como valores equivalentes se utilizarán la permeabilidad efectiva $\kappa^{e f}$ calculada mediante el método propuesto, y los valores que se obtienen del promedio aritmético $\left(\kappa_{A}\right)$ y el promedio armónico $\left(\kappa_{H}\right)$ del campo de permeabilidad, esto es:

$$
\begin{gathered}
\kappa_{\mathrm{A}}=\frac{\int_{\Omega} \kappa(\mathbf{x}) d V}{\int_{\Omega} d V}, \\
\kappa_{\mathrm{H}}=\left(\frac{\int_{\Omega} \kappa^{-1}(\mathbf{x}) d V}{\int_{\Omega} d V}\right)^{-1} .
\end{gathered}
$$

Las curvas de atenuación y dispersión para los distintos campos de permeabilidad son calculados utilizando un test de compresión desarrollado por Rubino [88].

Para el análisis propuesto se considerará una muestra con un alto grado de heterogeneidad en el campo de permeabilidad. Al igual que en el caso de rocas fracturadas, se 
supondrá que el medio es de tipo binario, es decir, constituido por dos tipos de textura: una matriz de baja permeabilidad y una distribución de manchas ("patches") de alta permeabilidad. Ambas texturas poseen un marcado contraste en la compresibilidad; el material más permeable correspondiente a las manchas posee mayor compresibilidad que la matriz. La distribución espacial de las manchas es de tipo fractal y puede generarse utilizando una función espectral de tipo von-Karman. Para mayores detalles de la aplicación de las funciones espectrales al estudio de medios porosos heterogéneos se refiere a los trabajos $[90,91,110]$. En la Fig. 3.7 se ha representado una muestra de $15 \mathrm{~cm}$ de lado, discretizada mediante una malla regular de $150 \times 150$ elementos. Las regiones de color negro corresponden a la matriz de baja permeabilidad y las de color blanco a las manchas de alta permeabilidad. Para este ejemplo supondremos que las permeabilidades de la matriz y las manchas son $2.69 \times 10^{-3} D$ y $3.458 D$, respectivamente. Para calcular la permeabilidad hidráulica efectiva $\kappa^{e f}$ basta imponer un gradiente hidráulico efectivo tal que $h^{e f}$ sea positiva.

La Fig. 3.8 muestra el complejo patrón de flujo resultante de la simulación numérica. La escala de colores es proporcional a la magnitud de $|\mathbf{q}|$. Comparando las Figs. 3.7 y 3.8 puede observarse una fuerte correlación entre el campo de flujo y la distribución de heterogeneidades de la muestra. Como era de esperar, los valores máximos de $|\mathbf{q}|$ se corresponden con zonas de alta permeabilidad.

La permeabilidad hidráulica efectiva se obtiene a partir del campo de flujo y del valor del gradiente efectivo, como se describió en la sección (3.6), dando como resultado $\kappa^{e f}=7.648 \times 10^{-3} D$. Por su parte, los valores de la media aritmética y la media armónica fueron calculados utilizando (3.68) y (3.69), obteniéndose: $\kappa_{A}=1.0738 D$ y $\kappa_{H}=3.903 \times 10^{-3} D$.

Utilizando el test de compresión desarrollado por Rubino [88] se calcularon las curvas de atenuación y dispersión para la muestra con campo de permeabilidad heterogénea y para los 3 campos homogéneos equivalentes $\kappa^{e f}, \kappa_{A}$ y $\kappa_{H}$. En la Fig. 3.9 se muestran las curvas en función de la frecuencia obtenidas en cada caso. La curva de atenuación está expresada en términos del valor inverso del factor de calidad $\left(1 / Q_{p}\right)$ y la curva de dispersión en términos de la velocidad de fase $V_{p}$. Como puede observarse, no existe un valor de permeabilidad equivalente que permita reproducir el comportamiento sísmico de la muestra en todo el rango de frecuencias. Este hecho permite concluir que la permeabilidad sísmica es un parámetro dinámico, es decir dependiente de la frecuencia de las ondas 


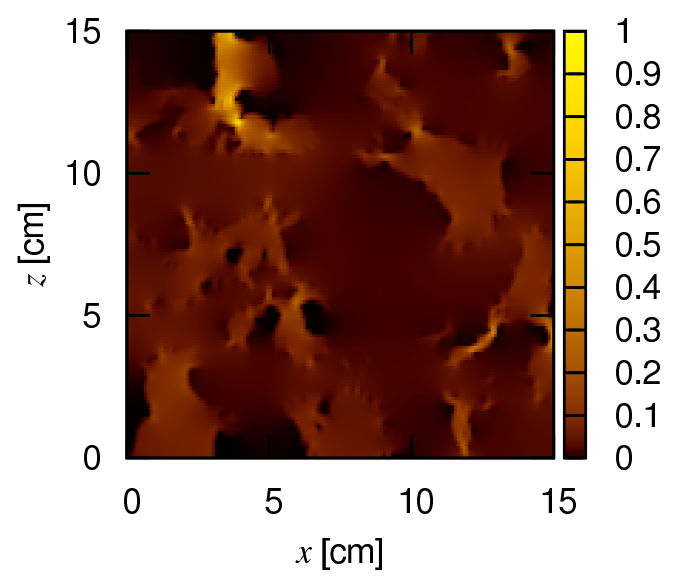

Figura 3.8: Módulo del campo de flujo (normalizado) resultante del experimento numérico.

sísmicas [74]. Sin embargo, como puede observarse en la Fig. 3.9, en el rango de bajas frecuencias la permeabilidad efectiva calculada con la metodología propuesta reproduce en forma adecuada la atenuación y dispersión de la muestra heterogénea. Este resultado permite concluir que la permeabilidad hidráulica efectiva coincide con la permeabilidad sísmica en este rango de frecuencias, lo que constituye un aporte importante ya que permite vincular parámetros sísmicos con propiedades hidráulicas del medio poroso. Los resultados del presente análisis fueron publicados en dos trabajos recientes [86, 87].

\subsection{Conclusiones}

En este Capítulo se ha presentado una técnica numérica para obtener relaciones constitutivas en medios porosos heterogéneos bidimensionales. Se presentaron las hipótesis y los métodos numéricos para la representación matemática de rocas fracturadas y se ilustró la aplicación de la técnica mediante el cálculo de las relaciones constitutivas para dos muestras de roca. Finalmente, la técnica numérica fue aplicada al cálculo de la permeabilidad efectiva en rocas con heterogeneidades de tipo fractal, resaltando la importancia de esta aplicación particular al estudio de las propiedades sísmicas de medios porosos heterogéneos. 

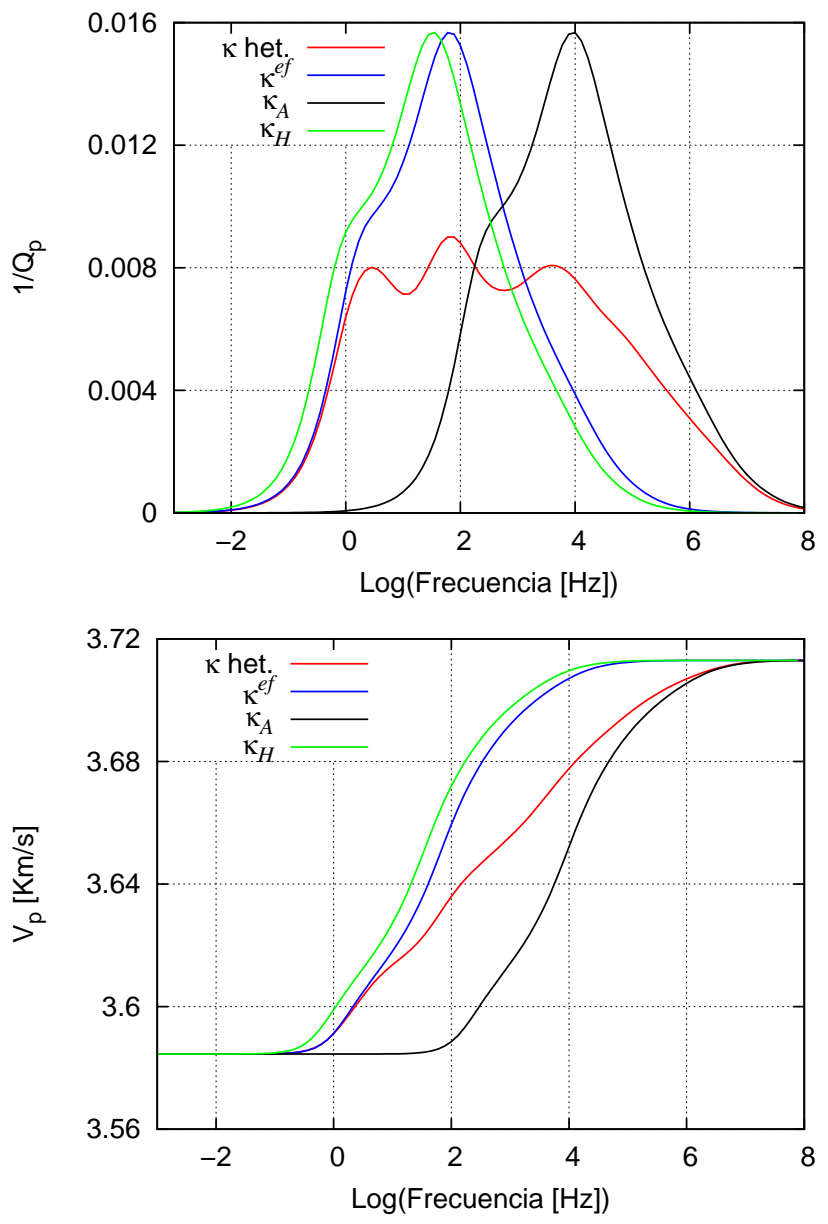

Figura 3.9: Curva del valor inverso del factor de calidad $1 / Q_{p}$ (arriba) y velocidad de fase $V_{p}$ (abajo) como función de la frecuencia para la muestra heterogénea de la Fig. 3.7 ( $\kappa$ het.) y para los campos de permeabilidad homogénea $\kappa^{e f}, \kappa_{A}$ y $\kappa_{H}$. 


\section{Capítulo 4}

\section{Diseño de experimentos numéricos 3D}

En este Capítulo se presenta una extensión al caso tridimensional del método numérico presentado en el Capítulo 3. Dado que la única complejidad adicional en el caso 3D radica en que se agrega una dimensión, se omiten los detalles del algoritmo, los cuales forman parte del Apéndice A. La aplicación del método es ilustrada mediante dos ejemplos. En el primero se obtienen y analizan las relaciones constitutivas de una muestra de roca fracturada tridimensional. En el segundo se efectúa un análisis comparativo de la conductividad hidráulica efectiva estimada mediante simulaciones 3D y 2D sobre una misma muestra de roca heterogénea con una distribución lognormal de conductividad hidráulica. La muestra 3D es generada mediante un algoritmo de bandas rotantes o tipo turning bands y para su representación bidimensional se considera un número de cortes extraidos de la misma. Las aplicaciones que se presentan en este Capítulo forman parte de los trabajos [41], [65], [70] y [71].

\subsection{Planteo matemático del experimento}

El experimento 3D será planteado en forma análoga al caso 2D. Comenzamos por considerar una muestra con forma de prisma rectangular como la que se ilustra en la Fig. 4.1. La misma ocupa el dominio $\Omega=\left[0, L_{x}\right] \times\left[0, L_{y}\right] \times\left[0, L_{z}\right]$ con frontera $\Gamma=$ $\Gamma^{L} \cup \Gamma^{R} \cup \Gamma^{F} \cup \Gamma^{B} \cup \Gamma^{D} \cup \Gamma^{U}$, y está orientada de modo que el eje $z$ positivo es antiparalelo a la dirección de la gravedad. Notar que en el caso 3D se agregan las caras $\Gamma^{F}$ y $\Gamma^{B}$ 1. Supondremos que la muestra se encuentra impermeabilizada en sus caras laterales $\Gamma^{L}, \Gamma^{R}, \Gamma^{F}$ y $\Gamma^{B}$, y sometida a alturas de presión constante en sus caras horizontales $\Gamma^{D}$ y $\Gamma^{U}$. Bajo estas condiciones, y asumiendo las mismas hipótesis que en el caso bidimensional, el problema diferencial que describe el flujo no saturado en la muestra 3D puede

\footnotetext{
${ }^{1}$ del inglés Front (delante) y Back (detrás).
} 

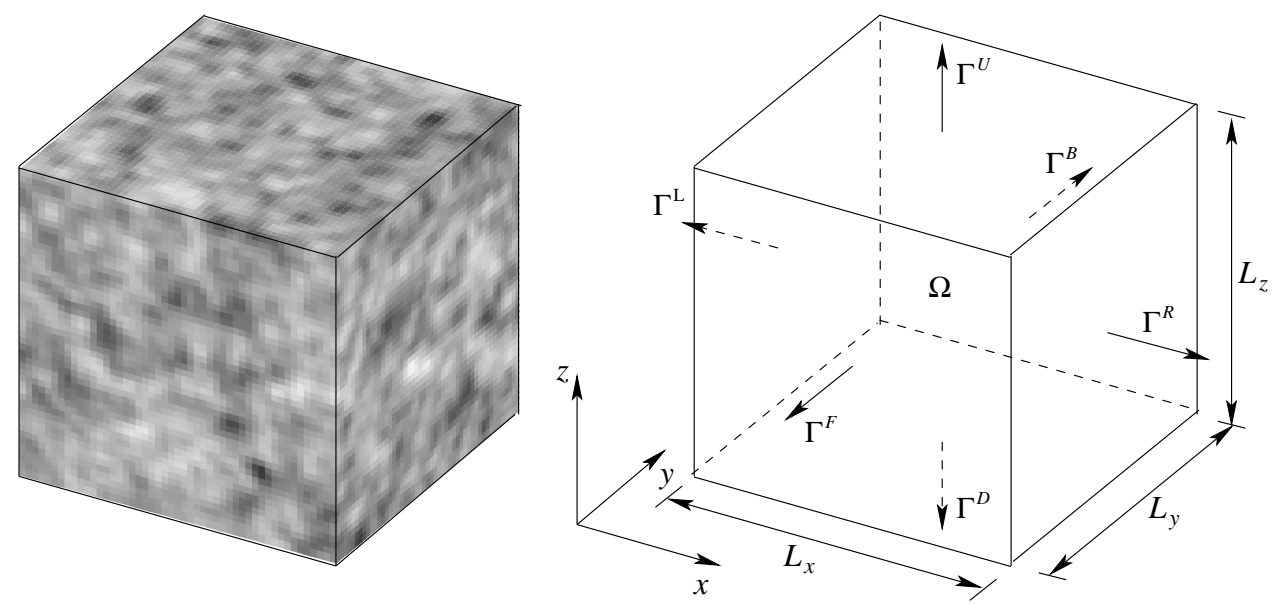

Figura 4.1: Muestra 3D de medio poroso (izquierda) y representación matemática de su dominio (derecha).

plantearse del siguiente modo: Hallar $(h, \mathbf{q}): \Omega \times \Omega \rightarrow R \times R^{3}$ tal que:

$$
\begin{aligned}
& \boldsymbol{\nabla} \cdot \mathbf{q}=0, \quad(x, y, z) \in \Omega, \\
& \mathbf{q}=-K(h) \boldsymbol{\nabla}(h+z), \quad(x, y, z) \in \Omega, \\
& h=h^{D}, \quad(x, y, z) \in \Gamma^{D}, \\
& h=h^{U}, \quad(x, y, z) \in \Gamma^{U}, \\
& \mathbf{q} \cdot \boldsymbol{\nu}=0, \quad(x, y, z) \in \Gamma^{L} \cup \Gamma^{R} \cup \Gamma^{F} \cup \Gamma^{B},
\end{aligned}
$$

siendo $\boldsymbol{\nu}$ el versor normal externo a la cara considerada. Como puede observarse, el problema (4.1)-(4.5) es equivalente a (3.1)-(3.5) y por tanto puede ser resuelto utilizando la misma metodología. En este caso el dominio es particionado mediante una malla de subdominios con forma de prisma rectangular, y para la formulación débil del problema se utilizan nuevamente los espacios de Raviart-Thomas-Nedelec de orden cero $\left(\mathrm{RTN}_{0}\right)$. Los detalles del algoritmo para la resolución numérica del problema (4.1)-(4.5) se han incluido en el Apéndice A. La implementación del algoritmo fue realizada mediante un código desarrollado en lenguaje Fortran.

\subsection{Cálculo de las relaciones constitutivas}

En esta sección se presentarán las expresiones discretas para el cálculo de las relaciones constitutivas en el caso 3D. Las expresiones no discretas (3.52), (3.53), (3.58) y (3.60) que hemos introducido en la sección (3.6) serán igualmente válidas, puesto que en las mismas 
no se hace referencia a la dimensión espacial. El experimento numérico para obtener las relaciones constitutivas es idéntico al descrito en la sección (3.2). Esto es, se establece un gradiente de presión imponiendo valores predeterminados de altura de presión $h^{D}$ y $h^{U}$ en las caras horizontales $\Gamma^{D}$ y $\Gamma^{U}$, y considerando flujo nulo en las caras laterales $\Gamma^{L} \cup \Gamma^{R} \cup \Gamma^{F} \cup \Gamma^{B}$.

El valor efectivo del contenido volumétrico de agua podrá obtenerse aproximando (3.53) mediante:

$$
\theta^{e f}=\frac{1}{L_{x} L_{y} L_{z}} \sum_{j=1, k=1, l=1}^{N_{x}, N_{y}, N_{z}} \theta\left(h_{j, k, l}\right) \Delta x_{j} \Delta y_{k} \Delta z_{l},
$$

siendo $h_{j, k, l}$ la altura de presión en el elemento $\Omega_{j, k, l}$. Análogamente, los valores residual y máximo de contenido de agua se aproximarán como:

$$
\begin{aligned}
& \theta_{r}^{e f}=\frac{1}{L_{x} L_{y} L_{z}} \sum_{j=1, k=1, l=1}^{N_{x}, N_{y}, N_{z}} \theta_{r, j, k, l} \Delta x_{j} \Delta y_{k} \Delta z_{l}, \\
& \theta_{s}^{e f}=\frac{1}{L_{x} L_{y} L_{z}} \sum_{j=1, k=1, l=1}^{N_{x}, N_{y}, N_{z}} \theta_{s, j, k, l} \Delta x_{j} \Delta y_{k} \Delta z_{l} .
\end{aligned}
$$

Luego, la saturación efectiva $S_{e}^{e f}$ podrá calcularse de la siguiente manera:

$$
S_{e}^{e f}=\frac{\theta^{e f}-\theta_{r}^{e f}}{\theta_{s}^{e f}-\theta_{r}^{e f}}=\frac{\sum_{j=1, k=1, l=1}^{N_{x}, N_{y}, N_{z}}\left[\theta\left(h_{j, k, l}\right)-\theta_{r, j, k, l}\right] \Delta x_{j} \Delta y_{k} \Delta z_{l}}{\sum_{j=1, k=1, l=1}^{N_{x}, N_{y}, N_{z}}\left[\theta_{s, j, k, l}-\theta_{r, j, k, l}\right] \Delta x_{j} \Delta y_{k} \Delta z_{l}} .
$$

El flujo efectivo $q^{e f}$ podrá obtenerse calculando en forma discreta el flujo neto a través de la cara inferior $\Gamma^{D}$ (ver ecuación $\left.(3.58)\right)$ :

$$
q^{e f}=\frac{\sum_{j=1, k=1}^{N_{x}, N_{y}} q_{j, k, 1}^{D} \Delta x_{j} \Delta y_{k}}{L_{x} L_{y}}
$$

mientras que la conductividad hidráulica efectiva se obtiene a partir de (3.60) y (4.10):

$$
K^{e f}\left(h^{e f}\right)=\frac{q^{e f}}{\left(\frac{h^{U}-h^{D}}{L_{z}}+1\right)}=\frac{\sum_{j=1, k=1}^{N_{x}, N_{y}} q_{j, k, 1}^{D} \Delta x_{j} \Delta y_{k}}{L_{x} L_{y}\left(\frac{h^{U}-h^{D}}{L_{z}}+1\right)} .
$$

La conductividad hidráulica saturada $K_{s}^{e f}$ puede calcularse en forma análoga a (4.11) considerando un valor arbitrario de altura de presión positiva. Finalmente $K_{r}^{e f}$ puede obtenerse a partir de (3.64) utilizando los valores estimados de $K^{\text {ef }}$ y $K_{s}^{e f}$.

Utilizando las expresiones definidas en esta sección pueden obtenerse todas las cantidades efectivas de la muestra correspondientes a la altura de presión efectiva $h^{e f}$. Las 
curvas $\theta^{e f}\left(h^{e f}\right)$ y $K^{e f}\left(h^{e f}\right)$ se obtienen repitiendo el experimento numérico para distintos valores de $h^{e f}$, tal como se realiza en las determinaciones experimentales de laboratorio.

Los cálculos que se han presentado en esta sección fueron implementados mediante el diseño de subrutinas Fortran acopladas al código de simulación 3D. En la sección siguiente se presentará un ejemplo del cálculo de relaciones constitutivas aplicado al caso particular de rocas fracturadas.

\subsection{Relaciones constitutivas para rocas fracturadas 3D}

Las fracturas 3D serán representadas por prismas rectangulares de sección cuadrada que se desarrollan en torno de los centros de fractura. Las fracturas se asumen paralelas a los planos cartesianos con una ubicación aleatoria dentro del dominio de simulación. La apertura $b$ y longitud $L$ de las fracturas nuevamente están relacionadas por la ley fractal (3.67). En la Fig. 4.2 se ha representado en forma esquemática una posible configuración espacial para 5 fracturas generadas mediante el método propuesto.

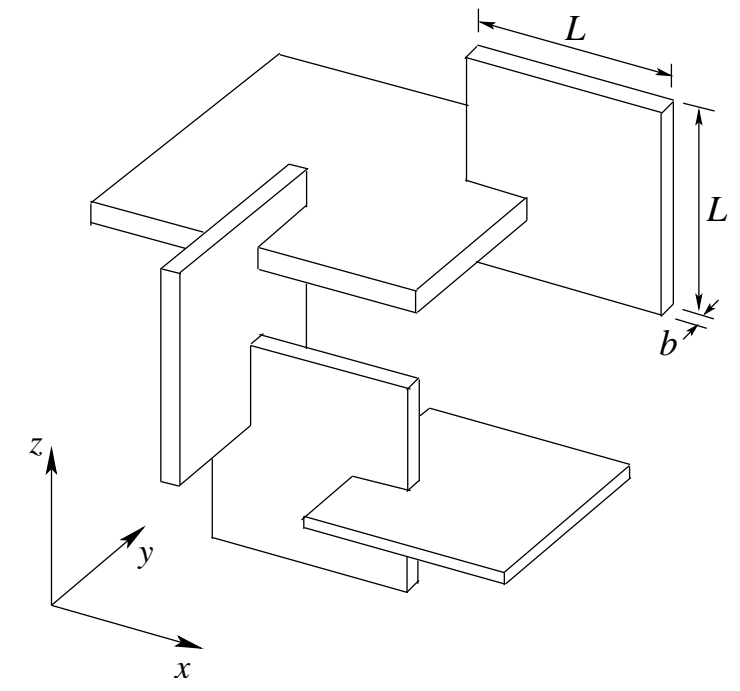

Figura 4.2: Representación esquemática de la distribución espacial de las fracturas y sus dimensiones.

Para el cálculo de las relaciones constitutivas se ha considerado una muestra cúbica de $10 \mathrm{~cm}$ de lado, discretizada mediante una partición regular de $50 \times 50 \times 50$ elementos. La red de fracturas posee un único valor de apertura $b=2 \mathrm{~mm}$, siendo los parámetros de la ley fractal $d=1.13$ y $c=0.02$. El número de fracturas generadas es 300 y para la caracterización hidráulica de la muestra se han utilizado nuevamente los parámetros 
de van Genuchten que se listan en la Tabla 3.1. En la Fig. 4.3 (a) se ha representado una vista del patrón de fracturas sobre la superficie de la muestra utilizando el mismo criterio de representación que en el caso 2D (fracturas en blanco y matriz en negro). Para el ejemplo considerado se calculó la curva de porosidad en función del volumen, la cual se muestra en la Fig. 4.3 (b). Al igual que en el caso bidimensional se observa un comportamiento errático para volúmenes pequeños, tendiendo a estabilizarse a medida que el volumen aumenta. Para el tamaño máximo puede considerarse que la muestra constituye un REV de roca fracturada.

(a)

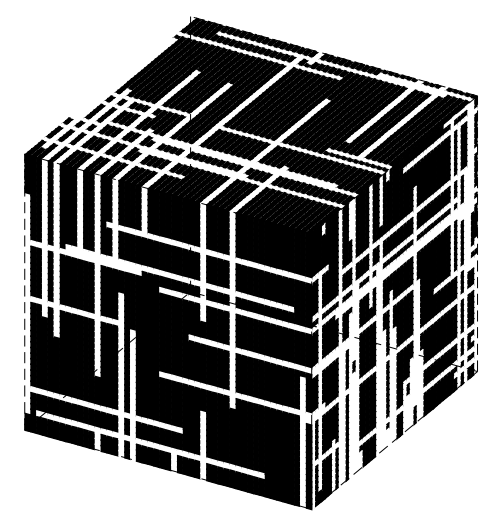

(b)

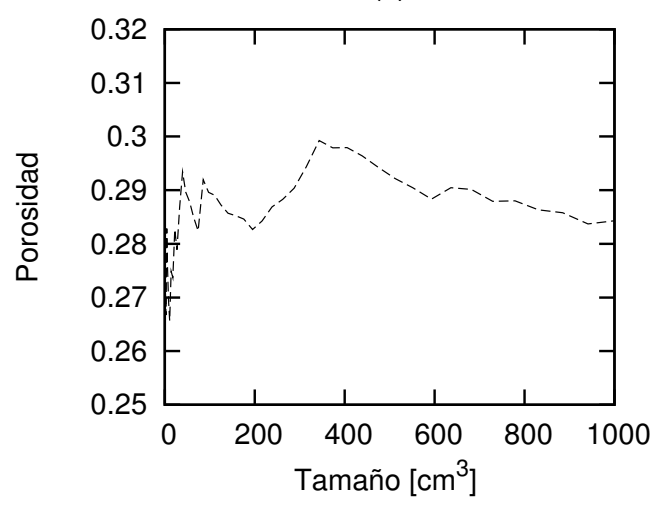

Figura 4.3: Muestra de roca fracturada (a) y porosidad en función del tamaño de la muestra (b).

Mediante el método propuesto se llevaron a cabo un total de 15 simulaciones para valores distintos de altura de presión efectiva $h^{\text {ef }}$ (uno de ellos es un valor positivo con el propósito de estimar la conductividad hidráulica saturada).

Para definir el gradiente de altura de presión en términos de $h^{e f}$ se ha utilizado el criterio descrito en la sección (3.7). Para cada valor de $h^{e f}$ se resolvió el problema (4.1)(4.5) y se estimaron los campos $h$ y q. A partir de ellos se calcularon $S_{e}^{e f}$ y $K_{r}^{e f}$ empleando las fórmulas (4.9) y (4.11).

La Fig. 4.4 muestra los valores simulados de $S_{e}^{e f}$ versus $h^{e f}$ (izquierda) y los de $K_{r}^{e f}$ como función de $S_{e}^{e f}$ (derecha). La figura incluye además las curvas de van Genuchten correspondientes al material de la matriz y las fracturas. Como puede observarse los resultados son comparables a los obtenidos en el caso 2D. Para valores pequeños de $\left|h^{e f}\right|$ 

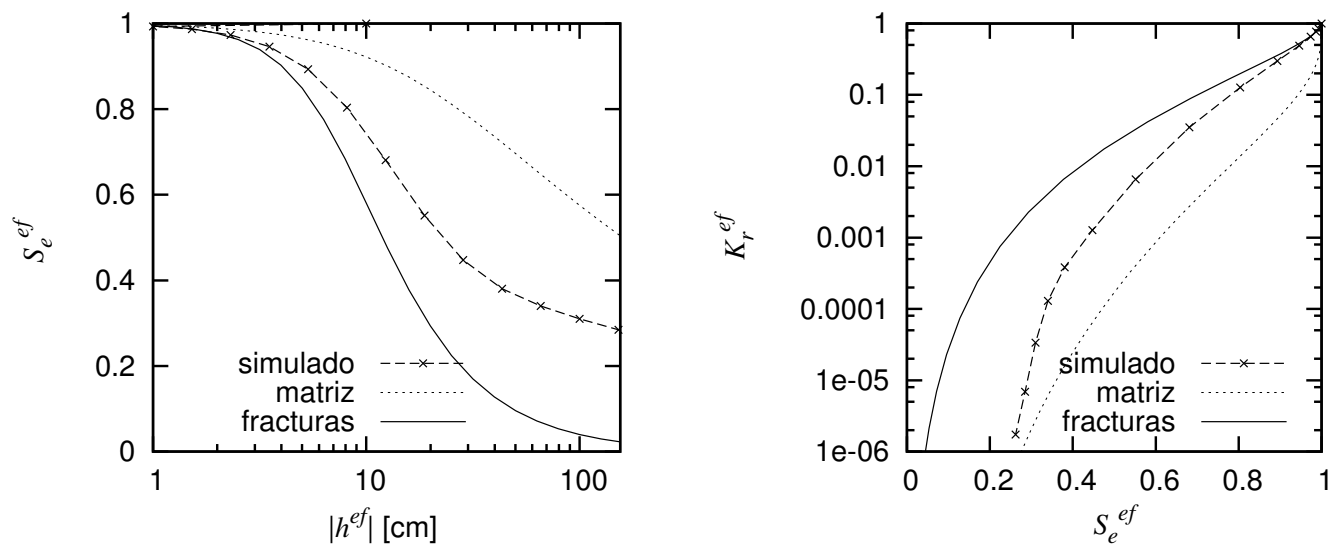

Figura 4.4: Saturación efectiva simulada (izquierda) y conductividad hidráulica relativa simulada (derecha).

(esto es, valores de saturación efectiva cercanos a 1) los valores simulados de $S_{e}^{e f}$ y $K_{r}^{e f}$ son similares a los valores de la curva de van Genuchten correspondientes a las fracturas. Este comportamiento sugiere que, cerca de la saturación, el flujo de agua en la muestra de roca está fuertemente determinado por las propiedades hidráulicas de las fracturas. A medida que aumenta $\left|h^{e f}\right|$, tanto $S_{e}^{e f}$ como $K_{r}^{e f}$ se apartan de la curva de las fracturas, acercándose en forma progresiva a la curva de la matriz. Esto se debe a que para valores muy bajos de saturación, la conductividad hidráulica de las fracturas es menor que la de la matriz (contrariamente a lo que ocurre para valores altos de saturación), resultando un flujo dominado exclusivamente por la matriz.

El comportamiento observado en las relaciones constitutivas simuladas resulta más evidente al analizar el campo de flujo resultante en cada simulación. En la Fig. 4.5 se han incluido tres gráficos del módulo del vector de flujo $|\mathbf{q}|$ normalizado para la muestra considerada. Los gráficos (b), (c) y (d) corresponden a tres valores distintos de $h^{\text {ef }}$ : -30, -45 y $-60 \mathrm{~cm}$, respectivamente. Nótese que para $h^{e f}=-30 \mathrm{~cm}$ los valores máximos de $|\mathbf{q}|$ se verifican en las fracturas, indicando su mayor aporte al flujo para este valor de altura de presión. Para $h^{e f}=-45 \mathrm{~cm}$ el flujo tiene una magnitud aproximadamente constante en toda la muestra. En este caso, tanto la matriz como las fracturas contribuyen del mismo modo al flujo total. Por último, para $h^{e f}=-60 \mathrm{~cm}$ la saturación en las fracturas es lo suficientemente pequeña como para que la conductividad en las mismas sea menor 
(a)

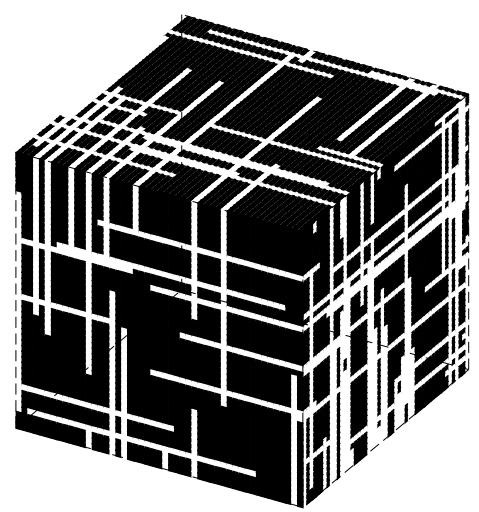

(c)

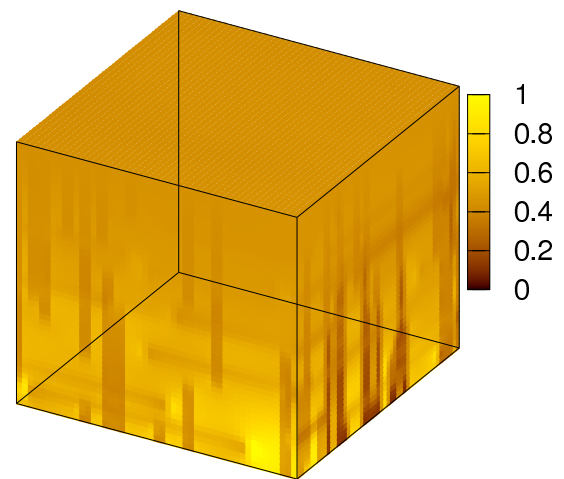

(b)

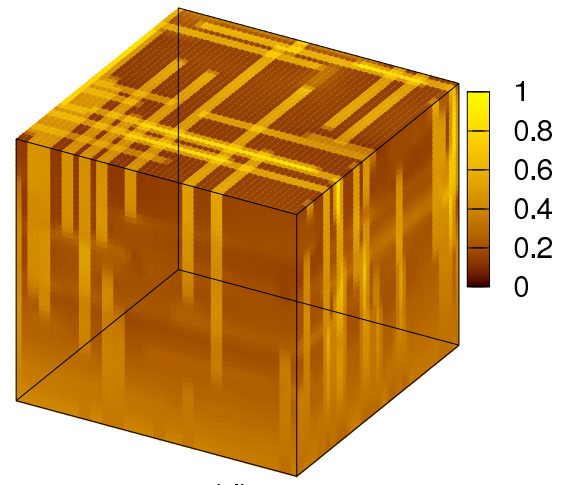

(d)

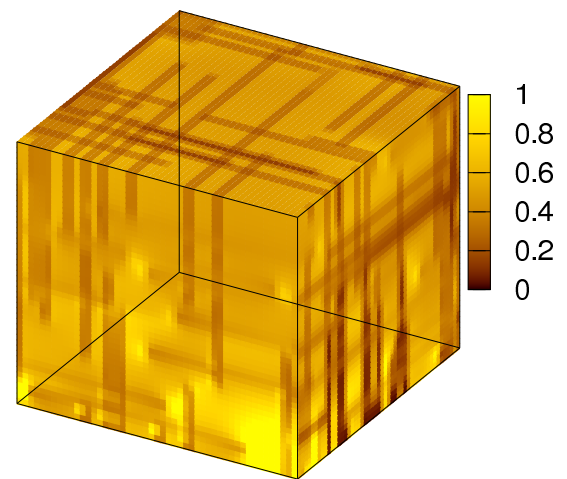

Figura 4.5: Muestra de roca fracturada (a) y gráficos del módulo de flujo normalizado para $h^{e f}=-30(\mathrm{~b}),-45$ (c) y $-60(\mathrm{~d}) \mathrm{cm}$.

que en la matriz, resultando esta última la principal contribuyente al flujo total en la muestra.

\subsection{Conductividad hidráulica en medios porosos heterogéneos $2 \mathrm{D}$ y $3 \mathrm{D}$}

Por cuestiones de índole práctica y costo computacional, las propiedades efectivas se obtienen generalmente bajo la hipótesis de que el medio poroso es bidimensional. En esta sección se analizará y discutirá la validez de esta hipótesis comparando los resultados de experimentos numéricos 3D y 2D sobre una muestra de roca heterogénea. En particular se estudiará la conductividad hidráulica efectiva en condiciones de saturación total, puesto que dicha cantidad suele presentar grandes variaciones al tratar con medios porosos heterogéneos. Notar que bajo estas condiciones, la ecuación (2.25) se reduce a 
$K^{e f}=K_{s}^{e f}$.

La muestra será generada utilizando una distribución lognormal, la cual resulta adecuada para representar las heterogeneidades presentes en la conductividad hidráulica [24]. El algoritmo utilizado para generar el campo heterogéneo es de tipo turning bands $[48,62]$. Este algoritmo permite generar campos heterogéneos con cierto grado de correlación espacial, como se verá en la sección siguiente.

La conductividad hidráulica efectiva $K^{e f}$ de la muestra será estimada utilizando el método numérico propuesto. El valor obtenido será comparado con los valores equivalentes dados por la media aritmética, la media geométrica y la media armónica, los cuales pueden calcularse directamente a partir del campo de conductividad de la muestra. Finalmente, con el propósito de estudiar el efecto de la dimensión espacial, se comparará la conductividad hidráulica efectiva de la muestra con las conductividades efectivas obtenidas aplicando el método propuesto a un número de cortes 2D de la muestra 3D. Este sencillo análisis permitirá determinar la validez de la aproximación 2D de la muestra considerada.

\subsubsection{Generación de la muestra de roca heterogénea}

En esta sección se decribe brevemente el procedimiento computacional para generar una muestra sintética de roca con una distribución lognormal de conductividad hidráulica utilizando un algoritmo de tipo turning bands o bandas giratorias. El nombre del algoritmo hace referencia a la técnica utilizada para generar un campo con una determinada correlación espacial. Para mayor detalle sobre los fundamentos de esta técnica y su implementación se refiere a [48] y [62].

Supondremos que la muestra de roca ocupa un dominio cúbico $\Omega$ de lado $L$, donde el campo de conductividad $K(\mathbf{x})$ es de tipo lognormal, esto es, el campo $Y(\mathbf{x})=\ln (K(\mathbf{x}))$ es de tipo gaussiano. Notar que hemos utilizado el símbolo $\mathbf{x}$ para hacer referencia a un punto arbitrario $(x, y, z)$ del dominio de la muestra. Esto se realiza a efectos de simplificar la notación.

Siguiendo a Desbarats [25] asumiremos que $Y(\mathbf{x})$ es estacionario y ergódico. Con estas hipótesis, el campo gaussiano queda definido por sus momentos de primer y segundo orden:

$$
E[Y(\mathbf{x})]=\alpha,
$$




$$
\begin{gathered}
\operatorname{Cov}[Y(\mathbf{x}), Y(\mathbf{x}+\mathbf{r})]=C(|\mathbf{r}|), \\
\operatorname{Var}[Y(\mathbf{x})]=\sigma^{2}
\end{gathered}
$$

donde $\alpha, C(|\mathbf{r}|)$ y $\sigma^{2}$ son, respectivamente, la media, la covarianza y la varianza del campo gaussiano. La función de covarianza se asume isotrópica y de tipo exponencial simple:

$$
C(r)=\sigma^{2} e^{-b r}
$$

siendo $b$ el inverso de la distancia de correlación y $r=|\mathbf{r}|$ la distancia entre dos puntos arbitrarios de la muestra.

Para implementar el algoritmo turning bands es necesario definir un número $N_{s}$ de semirrectas en $\mathbb{R}^{3}$, cuyo origen común sea un punto arbitrario y cuya orientación en el espacio esté uniformemente distribuida. Llamaremos $\mathbf{u}_{i}$ al versor directriz de la $i$-ésima semirrecta. A lo largo de la dirección dada por $\mathbf{u}_{i}$ se define una partición regular en bandas transversales de un ancho prefijado $\Delta \xi$ (en 3D estas bandas son prismas rectangulares) y se genera un proceso estacionario discreto de segundo orden con media cero y covarianza $C_{1}(\xi)$ dada por:

$$
C_{1}(\xi)=\frac{d}{d \xi}[\xi C(\xi)]=\sigma^{2}(1-b \xi) e^{-b \xi}
$$

siendo $\xi$ la distancia al origen medida sobre la semirrecta. Particionando el dominio de la muestra en elementos cúbicos $\Omega_{j}$ el proceso estacionario unidimensional permite definir en cada elemento, una cantidad $N_{s}$ de valores $z_{i}\left(\xi_{j}\right)=z_{i}\left(\mathbf{x}_{j} \cdot \mathbf{u}_{i}\right)$, con $i=1, . ., N_{s}$, siendo $\xi_{j}$ la distancia entre el origen común de las semirrectas y la proyección del punto $\mathbf{x}_{j}$ (posición del elemento $\Omega_{j}$ ) sobre la semirrecta de directriz $\mathbf{u}_{i}$. Finalmente, se asigna al elemento $\Omega_{j}$ el valor:

$$
Z\left(\mathbf{x}_{j}\right)=\frac{1}{\sqrt{N_{s}}} \sum_{i=1}^{N_{s}} z_{i}\left(\mathbf{x}_{j} \cdot \mathbf{u}_{i}\right) .
$$

De esta manera, el campo $Z$ resultante es estacionario de segundo orden e isótropo, cuya media es cero y su función de covarianza espacial está dada por (4.15) [62]. El paso siguiente es definir $Y\left(\mathbf{x}_{j}\right)$ en términos de $Z\left(\mathbf{x}_{j}\right)$ como:

$$
Y\left(\mathbf{x}_{j}\right)=\alpha+Z\left(\mathbf{x}_{j}\right)
$$

Dado que $Y$ es gaussiano, las estadísticas de $K$ pueden escribirse del siguiente modo:

$$
E[K(\mathbf{x})]=e^{\alpha+\sigma^{2} / 2}
$$



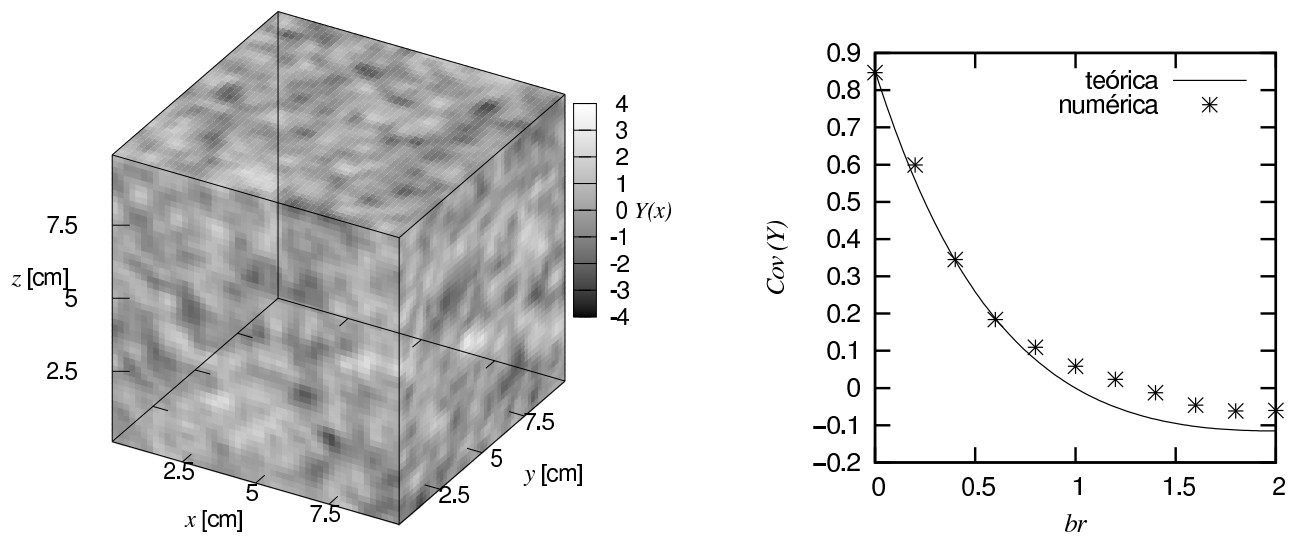

Figura 4.6: Realización 3D (izquierda) y gráfico de covarianza (derecha) para un campo $Y(\mathbf{x})$ generado utilizando el algoritmo turning bands.

$$
\operatorname{Var}[K(\mathbf{x})]=\left(e^{\sigma^{2}}-1\right) e^{2 \alpha+\sigma^{2}} .
$$

En la Fig. 4.6 se ilustra una realización 3D obtenida con el algoritmo descrito que fue implementado en lenguaje Fortran. Para este ejemplo se ha considerado una muestra de $10 \mathrm{~cm}$ de lado discretizada mediante una malla de $50 \times 50 \times 50$ elementos, y se utilizaron $N_{s}=10^{4}$ semirrectas uniformememente distribuidas, centradas en el origen de coordenadas. Las estadísticas del campo $Y(\mathbf{x})$ son $\alpha=0, \sigma^{2}=1 \mathrm{y} b=1 \mathrm{~cm}^{-1}$. El ancho de las bandas del algoritmo turning bands es de $0.4 \mathrm{~cm}$ (aproximadamente 62 bandas por línea). En la gráfica de la izquierda puede visualizarse la distribución de magnitudes de $Y$ sobre la superficie de la muestra. La gráfica de la derecha muestra, en línea continua, la función de covarianza teórica unidimensional dada por la ecuación (4.16), junto con los valores numéricos que se obtienen al calcular dicha covarianza en la dirección de uno de los ejes coordenados. Debido a la isotropía del campo las curvas de covarianza resultan similares si se las calcula a lo largo de cualquier dirección espacial. Como puede observarse, el algoritmo implementado puede generar con buena aproximación un campo gaussiano con una dada función de covarianza.

Finalmente, el campo de conductividad hidráulica $K(\mathbf{x})$ que verifica las estadísticas (4.19) y (4.20) se obtiene a partir de $Y(\mathbf{x})$ mediante:

$$
K(\mathbf{x})=e^{Y(\mathbf{x})}
$$

En la sección siguiente se obtendrá la conductividad hidráulica efectiva para una muestra 
generada mediante el algoritmo descrito.

\subsubsection{Cálculo de la conductividad hidráulica efectiva}

Para este ejemplo se ha calculado un campo de conductividad hidráulica $K\left(\mathbf{x}_{j}\right)$ utilizando la misma realización $Z\left(\mathbf{x}_{j}\right)$ generada para la muestra de la Fig. 4.6. Los parámetros estadísticos que representan al campo de conductividad hidráulica son $E[K(\mathbf{x})]=100 \mathrm{~cm} / \mathrm{d}$ y $\operatorname{Var}[K(\mathbf{x})]=10^{4} \mathrm{~cm}^{2} / \mathrm{d}^{2}$.

Con la técnica propuesta se calculó el valor efectivo de conductividad hidráulica para la muestra 3D en condiciones de saturación total. Con el fin de comparar el resultado se calcularon además las conductividades equivalentes dadas por la media aritmética $K_{\mathrm{A}}$, la media geométrica $K_{\mathrm{G}}$ y la media armónica $K_{\mathrm{H}}$. Si bien estas cantidades carecen de sentido físico, han sido ampliamente utilizadas como valores representativos de la conductividad hidráulica efectiva [25, 92]. Las medias $K_{\mathrm{A}}, K_{\mathrm{G}}$ y $K_{\mathrm{H}}$ se definen a partir del campo de conductividad hidráulica de la siguiente manera:

$$
\begin{gathered}
K_{\mathrm{A}}=\frac{\int_{\Omega} K(\mathbf{x}) d V}{\int_{\Omega} d V}, \\
K_{\mathrm{G}}=\exp \left(\frac{\int_{\Omega} \ln (K(\mathbf{x})) d V}{\int_{\Omega} d V}\right), \\
K_{\mathrm{H}}=\left(\frac{\int_{\Omega} K(\mathbf{x})^{-1} d V}{\int_{\Omega} d V}\right)^{-1} .
\end{gathered}
$$

El valor de conductividad hidráulica efectiva $K^{e f}$ calculado para el presente ejemplo fue de $107.32 \mathrm{~cm} / \mathrm{d}$, mientras que para los valores equivalentes se obtuvo: $K_{A}=$ $152.61 \mathrm{~cm} / \mathrm{d}, K_{G}=100 \mathrm{~cm} / \mathrm{d}$ y $K_{H}=65.17 \mathrm{~cm} /$ d. Nótese que el valor efectivo estimado para la muestra 3D es semejante al valor de la media geométrica. Esta semejanza es una característica propia de los campos heterogéneos lognormal [25] y en general no se verifica al tratar con otro tipo de heterogeneidad. Por ejemplo, en el caso de la roca fracturada de la sección (4.3) la conductividad hidráulica efectiva resultó $K^{e f}=98.58 \mathrm{~cm} / \mathrm{d}$, mientras que para los valores equivalentes se obtuvo: $K_{A}=209.71 \mathrm{~cm} / \mathrm{d}, K_{G}=20.02 \mathrm{~cm} / \mathrm{d}$ y $K_{H}=0.69 \mathrm{~cm} / \mathrm{d}$. Obsérvese que en este caso $K^{e f}$ y $K_{G}$ difieren casi en un orden de magnitud.

Para evaluar el efecto que produce la aproximación de la muestra 3D mediante una representación bidimensional, se han extraido de la muestra 50 cortes verticales transversales al eje $x$, y se llevó a cabo un análisis equivalente en 2D. La Fig. 4.7 muestra los 


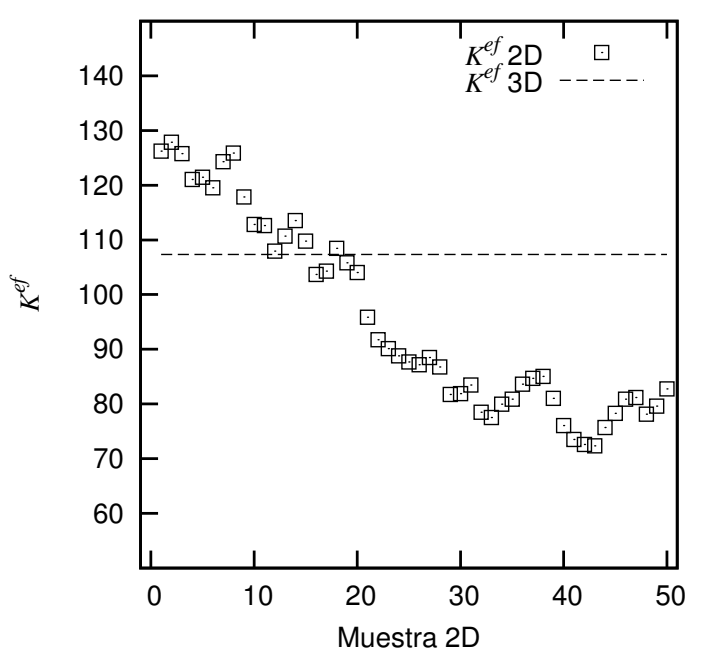

Figura 4.7: Conductividad hidráulica efectiva, expresada en $\mathrm{cm} / \mathrm{d}$, para la muestra 3D $\left(K^{e f}(3 D)\right)$ y para las 50 muestras $2 \mathrm{D}\left(K^{e f}(2 D)\right)$.

valores obtenidos para los 50 cortes $2 \mathrm{D}$ junto con el valor calculado para la muestra 3D. Puede observarse que los valores correspondientes a los cortes no coinciden en general con el valor efectivo de la muestra, difiriendo de este en algunos casos hasta en un $25 \%$. Más aún, si se considera como valor representativo de las 50 muestras 2D el promedio de las conductividades hidráulicas efectivas $(95.39 \mathrm{~cm} / \mathrm{d})$, este valor resulta inferior al valor efectivo obtenido para la muestra 3D $(107.32 \mathrm{~cm} / \mathrm{d})$.

Los valores equivalentes de conductividad hidráulica $K_{A}, K_{G}$ y $K_{H}$ también fueron calculados para cada uno de los cortes 2D, y los resultados se muestran en la Fig. 4.8 junto con los correspondientes valores efectivos. Como puede observarse, al igual que en el caso 3D la media geométrica es la que más se aproxima al valor efectivo. Sin embargo, en este caso, todos los valores efectivos resultan levemente inferiores a la media geométrica.

Para finalizar, en la Fig. 4.9 se muestra el módulo del vector de flujo normalizado para simulaciones 2D y 3D de la muestra. La gráfica de la izquierda corresponde a un corte vertical en el centro de la muestra 3D, donde puede observarse el patrón de flujo resultante. El campo de flujo de la muestra 2D correspondiente a este corte se muestra en el gráfico derecho de la Fig. 4.9. Como era de esperar los patrones de flujo son similares pero no idénticos, ya que los caminos preferenciales de flujo en el caso 3D son más complejos y no están limitados a un único plano.

Este sencillo ejemplo ha permitido demostrar la importancia de considerar medios 3D para calcular las propiedades efectivas de un medio poroso. La hipótesis de que 


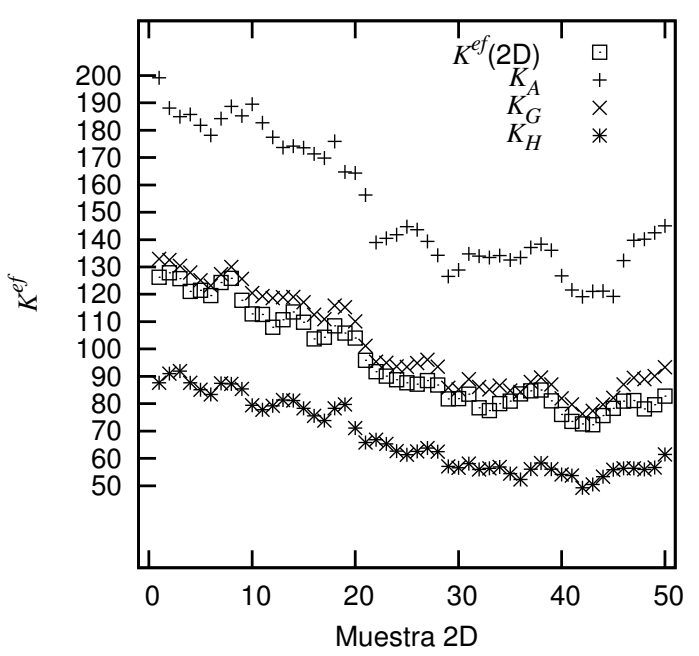

Figura 4.8: Conductividad hidráulica efectiva, expresada en cm/d, para los 50 cortes 2D de la muestra $\left(K^{e f}(2 D)\right)$ y sus respectivos valores equivalentes $K_{A}, K_{G}$ y $K_{H}$.
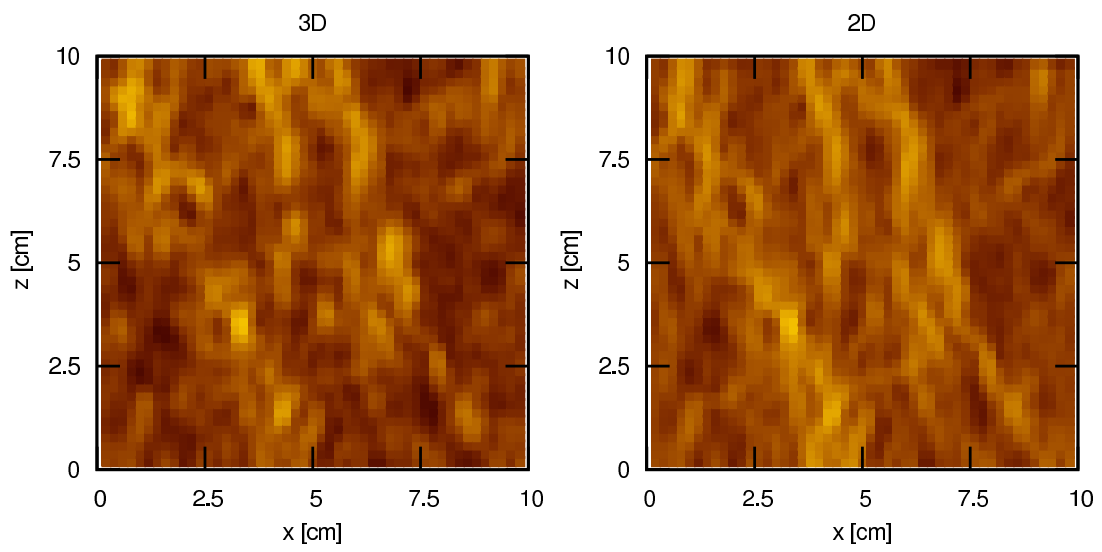

Figura 4.9: Módulo del vector de flujo 3D (izquierda) y 2D (derecha). Las tonalidades más claras corresponden a mayores intensidades. 
una muestra se puede representar por un dominio 2D limita los caminos preferenciales de flujo. Estas variaciones en el patrón de flujo modifican sensiblemente el valor de la conductividad efectiva que se obtiene mediante una representación 2D. Los errores que se cometen en la representación 2D pueden ser significativos y dependerán esencialmente del patrón espacial que presente la heterogeneidad.

Resulta importante remarcar que en el ejemplo presentado se ha trabajado con una única muestra de roca pues el objetivo era analizar la diferencia entre las representaciones 2D y 3D del medio poroso. Sin embargo, cualquier caracterización precisa de las propiedades hidráulicas debe realizarse en términos estadísticos sobre la base de un gran número de realizaciones.

\subsection{Conclusiones}

En este Capítulo se ha presentado una extensión al caso tridimensional de la técnica numérica propuesta en el Capítulo 3 para el cálculo de relaciones constitutivas en medios porosos heterogéneos. El método ha sido ilustrado mediante dos ejemplos. En el primero se han obtenido las relaciones constitutivas para una muestra de roca fracturada generada mediante un método análogo al utilizado en el caso 2D. Las relaciones constitutivas calculadas para este ejemplo presentan un comportamiento cualitativamente similar al caso 2D. En el segundo ejemplo se efectuó un análisis comparativo entre la conductividad hidráulica efectiva calculada para una muestra de roca 3D con heterogeneidades de tipo lognormal y las correspondientes conductividades calculadas para muestras 2D representativas de la muestra 3D. A partir de este ejemplo se ha podido concluir que los valores efectivos 2D pueden diferir del valor efectivo de la muestra dependiendo del corte que se considere, lo que muestra la importancia de considerar medios 3D para la determinación de las propiedades efectivas. 


\section{Capítulo 5}

\section{Modelos constitutivos analíticos para rocas fracturadas}

En este Capítulo se presenta una derivación teórica de un modelo constitutivo válido para la caracterización del flujo bifásico (aire y agua) en rocas fracturadas. El modelo conceptual asume que la red de fracturas puede representarse por un objeto fractal denominado carpeta de Sierpinski. Utilizando relaciones capilares clásicas y leyes de flujo se derivan expresiones analíticas cerradas para la saturación efectiva y la permeabilidad relativa del aire y del agua. Cuando se asume que el aire se encuentra en reposo, las curvas de saturación efectiva y permeabilidad relativa del agua conforman un modelo constitutivo válido para la descripción del flujo no saturado de agua (flujo monofásico). Este modelo es validado mediante la comparación con el modelo clásico de Burdine [15] y mediante el ajuste con las relaciones constitutivas simuladas en los Capítulos 3 y 4 para rocas fracturadas. Los modelos que se presentan en este Capítulo forman parte de los trabajos [66] y [69].

\subsection{Introducción}

En los Capítulos 3 y 4 se han presentado técnicas numéricas que permiten obtener las relaciones constitutivas de rocas heterogéneas sintéticas. Estas técnicas resultan particularmente útiles al tratar con rocas fracturadas, puesto que para este tipo de medios la determinación de la conductividad hidráulica mediante experimentos de laboratorio es muy dificultosa y como consecuencia los datos disponibles son muy escasos [59, 103]. Otra alternativa a la medición directa es el empleo de fórmulas predictivas que permiten obtener la curva de conductividad hidráulica a partir de la curva de saturación, cuya determinación en laboratorio no presenta mayores inconvenientes. Las fórmulas predictivas de $K(S)$ son ampliamente utilizadas, y entre ellas se destacan las de Burdine [15] y Mualem [73] cuyas expresiones fueron presentadas en el Capítulo 2. Estas fórmulas clásicas han sido desarrolladas para medios porosos de estructura granular (sedimentarios), no existiendo en la literatura fórmulas equivalentes aplicables a rocas fracturadas. 
A pesar de esto, la fórmula de Burdine ha sido utilizada por Liu y Bodvarsson [59] y por Guarracino y Quintana [42] para predecir la conductividad hidráulica en rocas fracturadas empleando distintas expresiones para la curva de saturación. La elección del modelo conceptual de Burdine se basa en la simplicidad de la geometría poral que resulta más adecuada para representar el flujo en fracturas.

En este Capítulo se propone un nuevo modelo conceptual para la representación de un REV de roca fracturada. El modelo se basa en la hipótesis de que el patrón de fracturas del REV es de tipo fractal. La autosimilitud es una propiedad característica de los objetos fractales, que ha sido observada en redes de fracturas por varios autores [104, 77, 6, 9]. Para representar la red de fracturas se utiliza la carpeta de Sierpinski. Este objeto fractal ha permitido obtener resultados muy satisfactorios en la descripción del contenido de agua y la conductividad hidráulica de medios porosos granulares. Basados en las propiedades geométricas de la carpeta, Tyler y Wheatcraft [106] han obtenido curvas de contenido de agua y han relacionado la dimensión fractal con la textura del suelo. Para el caso particular de rocas fracturadas, Guarracino [39] utiliza una carpeta de Sierpinski para obtener expresiones cerradas para la curva del contenido de agua. Una extensa revisión sobre teorías, métodos, modelos matemáticos y preguntas abiertas acerca de las propiedades del flujo y transporte en medios porosos fractales puede encontrarse en $[8,26,112]$.

Al tratar con el problema del flujo bifásico inmiscible de agua y aire bajo la aproximación del continuo, la hipótesis de Richards deja de ser válida. En este caso, ambas fases pueden fluir en forma simultánea, y los vectores de flujo $\mathbf{q}_{w}$ y $\mathbf{q}_{a}$ se relacionan con las presiones de las fases respectivas $p_{w}$ y $p_{a}$ a través de una ley de Buckingham-Darcy (ver ecuación (2.29)). Estas ecuaciones suelen expresarse en términos del gradiente de presión (en lugar del gradiente de altura de presión) y de la permeabilidad relativa (en lugar de la conductividad hidráulica). Asimismo, las saturaciones de agua y de aire se suelen expresar en función de la presión capilar $p_{c}$ en lugar de la altura de presión.

A partir de establecer un criterio de ocupación de ambas fases en la muestra de roca fracturada, se derivan expresiones analíticas cerradas para las curvas de saturación efectiva en función de la presión capilar efectiva. Asumiendo además que ambas fases pueden fluir en forma simultánea y utilizando la ley de flujo entre dos placas paralelas se obtienen expresiones analíticas cerradas para las curvas de permeabilidad relativa del agua y del aire. Estas expresiones conforman un modelo constitutivo válido para la descripción 
del flujo bifásico en rocas fracturadas que depende de 5 parámetros independientes: la dimensión fractal de la carpeta de Sierpinski, las aperturas máxima y mínima de las fracturas y las saturaciones efectivas de emergencia para el flujo del agua y del aire. En particular, si se asume que el aire se encuentra en reposo, las curvas de saturación efectiva y de permeabilidad relativa del agua conforman un modelo constitutivo válido para la descripción del flujo no saturado de agua. Este modelo es validado mediante su comparación con el modelo clásico de Burdine y mediante el ajuste con las relaciones constitutivas simuladas en los Capítulos 3 y 4.

\subsection{Descripción de la red de fracturas}

Para derivar los modelos constitutivos consideraremos un volumen representativo elemental (REV) de roca fracturada. El REV será conceptualizado como un cubo de lado $a$ formado por una matriz impermeable y una red de fracturas que constituye su espacio poral (ver Fig. 5.1). Este modelo es similar al modelo clásico de tubos capilares rectos, donde el medio poroso granular se sustituye por un arreglo de tubos paralelos a la dirección de flujo y cuya sección transversal es variable (ver Fig. $2.6(a)$ ). Las fracturas se asumen paralelas a la dirección del flujo y su patrón de distribución se describe mediante una carpeta de Sierpinski. Este modelo de roca fracturada es idéntico al utilizado por Guarracino [39] para derivar una curva de contenido de agua.

La carpeta de Sierpinski que describe la distribución espacial de las fracturas se genera mediante un algoritmo recursivo [106, 105]. Este algoritmo se aplica a un cuadrado de lado $a$ correspondiente a una sección transversal del REV. El primer nivel de recursión se obtiene al subdividir el cuadrado original en $b_{1}^{2}$ cuadrados elementales de lado $x_{1}=a / b_{1}$. El patrón de fracturas se genera removiendo una cantidad $l_{1}$ de estos cuadrados de manera que formen una red de fracturas de apertura $x_{1}$ como se muestra en la Fig. 5.2. Para que la distribución de fracturas sea autosimilar, el patrón elegido debe repetirse en cada nivel de recursión. Para un nivel de recursión arbitrario $i$, el cuadrado original se subdivide en $b_{i}^{2}$ cuadrados elementales de lado $x_{i}=a / b_{i}$, siendo $b_{i}=b_{1}^{i}$. Puede demostrarse que en cada instancia de recursión la carpeta de Sierpinski verifica la siguiente relación [61]:

$$
b_{i}^{2}-l_{i}=b_{i}^{D}
$$

donde $l_{i}$ es el número de cuadrados de lado $x_{i}$ necesarios para cubrir el área total ocupada por las fracturas de apertura $X \geq x_{i}$, y $D \in(1,2)$ es la dimensión fractal de la carpeta. 


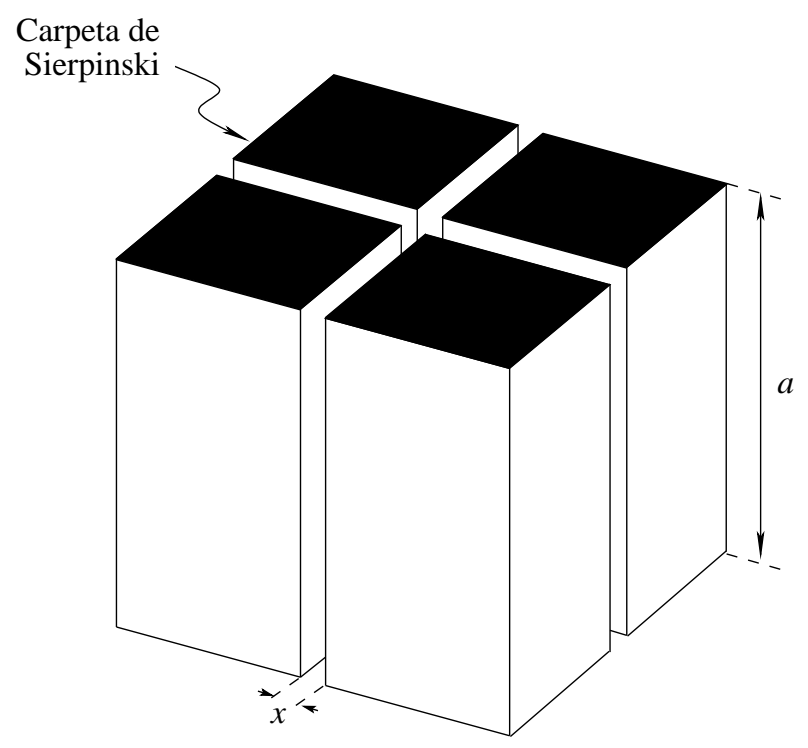

Figura 5.1: Volumen representativo elemental (REV) de roca fracturada.

Con el objeto de ilustrar el proceso recursivo descrito para la generación de la red de fracturas, se muestran en la Fig. 5.2 dos niveles de recursión para una carpeta de Sierpinski de dimensión fractal $D=1.84\left(b_{1}=7\right.$ y $\left.l_{1}=13\right)$.
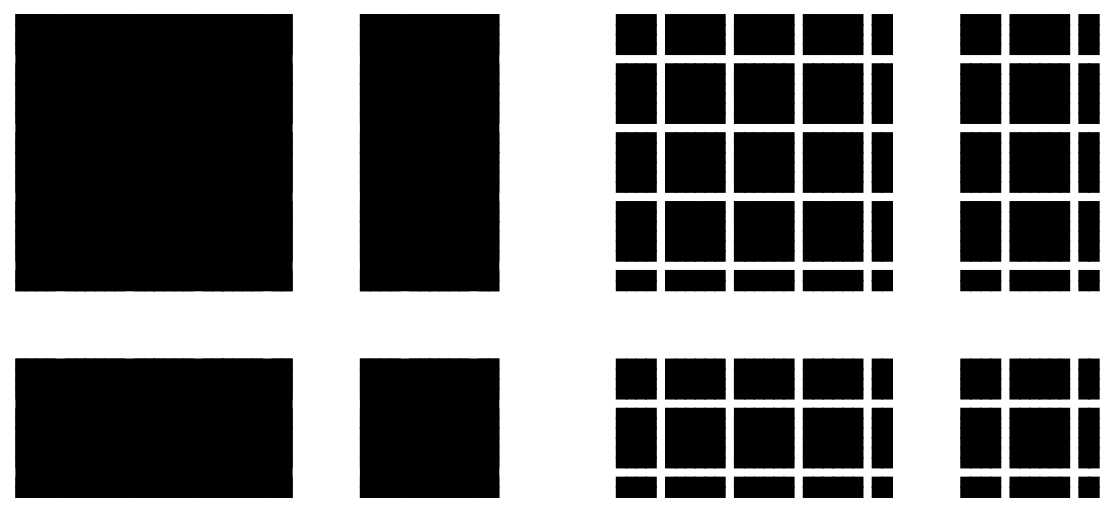

Figura 5.2: Dos niveles de recursión de una carpeta de Sierpinski con dimensión fractal $D=1.84$.

Para un valor arbitrario de $l$, el área ocupada por las fracturas de apertura $X \geq x$ está dada por:

$$
A(X \geq x)=x^{2} l
$$

donde $x^{2}$ representa el área de un cuadrado elemental. Reemplazando la ecuación (5.1) en (5.2) se obtiene la siguiente expresión para $A(X \geq x)$ en términos de los parámetros 
de la carpeta de Sierpinski:

$$
A(X \geq x)=a^{2}\left(1-a^{D-2} x^{2-D}\right)
$$

expresión que resulta válida para $x \leq a$.

El área ocupada por las fracturas cuya apertura se encuentra en el rango $(x, x+d x)$ se obtiene diferenciando la ecuación (5.3) con respecto a $x$ :

$$
-d A=(2-D) a^{D} x^{1-D} d x .
$$

El signo negativo en la ecuación (5.4) implica que el área cubierta por fracturas disminuye con el aumento de la apertura [69].

Es importante destacar que la carpeta de Sierpinski constituye un patrón geométrico que es autosimilar a toda escala menor que el tamaño inicial de la carpeta. Si el algoritmo recursivo se repitiera infinitas veces, la apertura de las fracturas más pequeñas tendería a cero, y según la ecuación (5.3) las fracturas ocuparían el volumen completo del REV. Sin embargo en medios reales, el comportamiento fractal solo es válido en un rango finito de escalas limitadas por valores de corte superior e inferior [2]. Para obtener una representación más realista consideraremos valores de corte máximo $x_{\text {máx }}$ y mínimo $x_{\text {mín }}$ para las aperturas de las fracturas de la red. Estos valores estarán definidos a partir de las aperturas máxima y mínima observadas en la muestra de roca. El corte inferior $x_{\text {mín }}$ resulta difícil de determinar experimentalmente. Comúnmente se le asigna un valor en función de ciertas consideraciones prácticas, como por ejemplo la mínima escala de resolución observable $[2,8]$.

En el análisis de la dimensión fractal de la carpeta de Sierpinski presentado en [39], se concluye que los valores de $D$ cercanos a 2 corresponden a medios levemente fracturados (baja densidad de fracturas y aperturas pequeñas) y los valores cercanos a 1 a medios con un alto grado de fracturación (alta densidad de fracturas y aperturas grandes). Sin embargo, la dimensión fractal no es suficiente para describir en forma unívoca la geometría del medio poroso. En el presente estudio, la geometría de un medio fracturado queda unívocamente determinada por la dimensión fractal de la carpeta de Sierpinski, las aperturas máxima y mínima, y la densidad de fracturas en el primer nivel de recursión [80].

En la sección siguiente, el modelo conceptual de roca fracturada que hemos presentado será utilizado para derivar un modelo constitutivo válido para flujo bifásico. 


\subsection{Modelo constitutivo bifásico}

En la mayoría de las aplicaciones prácticas de hidrogeología y ciencias del suelo relacionadas con medios parcialmente saturados se supone que la fase gaseosa (aire) permanece a presión constante (presión atmosférica) por lo que el problema se reduce a la consideración del movimiento del agua exclusivamente [7]. Como se ha mencionado en el Capítulo 2 a esta aproximación se la denomina aproximación de Richards y ha demostrado ser una buena alternativa para la descripción del flujo de agua en la zona no saturada del suelo [20]. Sin embargo, la descripción del flujo de la fase gaseosa resulta de importancia en problemas tales como el transporte de componentes orgánicos volátiles o la remediación del suelo mediante extracción de vapor [96, 32]. En estos casos debe considerarse un modelo bifásico que permita describir el flujo simultáneo de agua y aire en el medio poroso.

Las ecuaciones que describen el flujo bifásico bajo la aproximación del continuo requieren del conocimiento de las relaciones constitutivas entre las saturaciones, las permeabilidades relativas y las presiones de ambas fases. En esta sección se derivarán expresiones analíticas para las curvas de saturación efectiva y de permeabilidad relativa, válidas para la descripción del flujo inmiscible de agua y de aire en rocas fracturadas. Para la representación del patrón de fracturas se utilizará el modelo conceptual de roca fracturada que hemos introducido en la sección anterior. En primer lugar se establecerá un criterio de ocupación de las fases dentro del REV utilizando relaciones capilares clásicas. Luego se derivarán las expresiones analíticas para las saturaciones efectivas y las permeabilidades relativas de ambas fases. El modelo constitutivo propuesto ha sido publicado en un trabajo reciente [69].

\subsubsection{Criterio de ocupación de las fases}

Para derivar las relaciones constitutivas es necesario establecer el modo en que el aire y el agua se distribuyen dentro de la red de fracturas del REV. Asumiendo que el aire y el agua se encuentran a presiones efectivas constantes $p_{a}^{e f}$ y $p_{w}^{e f}$, respectivamente, es posible definir una presión capilar efectiva $p_{c}^{e f}$ a partir de la siguiente relación [7]:

$$
p_{c}^{e f}=p_{a}^{e f}-p_{w}^{e f}=\frac{2 \sigma \cos (\beta)}{x^{e f}},
$$

donde $\sigma$ es la tensión superficial, $\beta$ es el ángulo de contacto entre las dos fases, y $x^{e f}$ es una apertura efectiva de la red de fracturas. La ecuación (5.5) solo es válida en condiciones 
de equilibrio capilar y se obtiene a partir de (2.21) asumiendo una geometría poral de paredes planas paralelas. Luego, para un dado valor de la presión capilar efectiva $p_{c}^{e f}$, la ecuación (5.5) define una fractura de apertura $x^{e f}$ para la cual el aire y el agua están en equilibrio. Para cualquier otro valor de apertura $x$ el fluido ocupante está fluyendo.

El problema del flujo bifásico en el medio poroso fracturado puede separarse en dos problemas de flujo monofásico. Para ello asumiremos que las fracturas están ocupadas completamente por aire o por agua según el criterio propuesto por Pruess y Tsang [82]. El criterio de ocupación de fase de una fractura de apertura $x$ está regida por la presión capilar local $p_{c}=2 \sigma \cos (\beta) / x$. En una fractura de apertura $x$, el aire y el agua estarán en equilibrio solo cuando la presión capilar efectiva $p_{c}^{e f}$ coincida con la presión capilar local $p_{c}$. Si $p_{c}^{e f}$ es mayor que $p_{c}$, la diferencia $p_{a}^{e f}-p_{w}^{e f}$ (supuesta constante en todo el REV) resulta superior a la que se corresponde con el equilibrio $\left(p_{c}\right)$, por lo que el aire desplazará al agua ocupando todo el volumen de la fractura. Inversamente, si $p_{c}^{e f}$ es menor que $p_{c}$, la diferencia de presiones $p_{a}^{e f}-p_{w}^{e f}$ está por debajo de la presión de equilibrio y en este caso el agua desplaza al aire ocupando por completo la fractura.

Resulta importante aclarar que este criterio de ocupación ignora el proceso de flujo de películas delgadas adheridas a las paredes de la fractura cuando ésta se encuentra parcialmente saturada (film flow). Este proceso podría constituir un aporte importante en el flujo no saturado en rocas fracturadas [78]. Para los casos en que el flujo en láminas delgadas sea significativo, el modelo propuesto no logrará una predicción precisa de las relaciones constitutivas del medio. El modelo también ignora posibles efectos de la fase líquida, como el agua que puede quedar sujeta a rugosidades de las paredes por efecto de fuerzas de adsorción [82].

A partir de la ecuación (5.5) y del criterio de ocupación adoptado se concluye que todas las fracturas de apertura $x<x^{e f}$ están completamente saturadas de agua mientras que las de apertura $x>x^{e f}$ están ocupadas por aire. El rango de aperturas en el REV es $x_{\text {mín }} \leq x \leq x_{\text {máx }}$, luego la ecuación (5.5) es válida para el rango de presiones capilares efectivas $p_{c, \text { mín }} \leq p_{c}^{e f} \leq p_{c \text {,máx }}$, siendo $p_{c \text {,mín }}=2 \sigma \cos (\beta) / x_{\text {máx }} \mathrm{y} p_{c, \text { máx }}=2 \sigma \cos (\beta) / x_{\text {mín }}$.

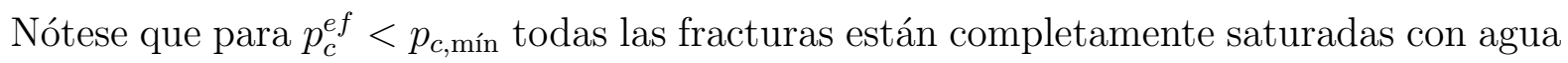
y el flujo de aire es nulo. Inversamente, todas las fracturas estarán saturadas con aire cuando $p_{c}^{e f}>p_{c \text {,máx }}$, resultando nulo el flujo de agua. 


\subsubsection{Saturaciones efectivas}

Según el criterio de ocupación que hemos adoptado, para un dado valor de presión capilar efectiva $p_{c}^{e f}$ todas las fracturas de apertura $x<x^{e f}$ estarán ocupadas por agua, mientras que las de apertura $x>x^{e f}$ estarán ocupadas por aire. Teniendo en cuenta la geometría del REV ilustrada en la Fig. 5.1, las saturaciones efectivas de agua y aire $\left(S_{e, w}\right.$ y $\left.S_{e, a}\right)$ estarán dadas por:

$$
S_{e, w}=\frac{\int_{x_{\min }}^{x^{e f}} a d A}{\int_{x_{\min }}^{x_{\operatorname{máx}}} a d A}, \quad S_{e, a}=\frac{\int_{x^{e f}}^{x_{\operatorname{máx}}} a d A}{\int_{x_{\min }}^{x_{\operatorname{máx}}} a d A} .
$$

Utilizando la expresión de $d A$ dada por (5.4) en las ecuaciones (5.6) e integrando, las saturaciones efectivas resultan:

$$
S_{e, w}=\frac{\left(x^{e f}\right)^{2-D}-x_{\operatorname{mín}}^{2-D}}{x_{\text {máx }}^{2-D}-x_{\operatorname{mín}}^{2-D}}, \quad S_{e, a}=\frac{x_{\text {máx }}^{2-D}-\left(x^{e f}\right)^{2-D}}{x_{\text {máx }}^{2-D}-x_{\text {mín }}^{2-D}} .
$$

Estas últimas pueden expresarse en términos de la presión capilar efectiva y de las presiones capilares máxima y mínima definidas en la sección anterior:

$$
S_{e, w}\left(p_{c}^{e f}\right)=\frac{\left(p_{c}^{e f}\right)^{D-2}-p_{c, \text { máx }}^{D-2}}{p_{c, \text { mín }}^{D-2}-p_{c, \text { máx }}^{D-2}}, \quad S_{e, a}\left(p_{c}^{e f}\right)=\frac{p_{c, \text { mín }}^{D-2}\left(p_{c}^{e f}\right)^{D-2}}{p_{c, \text { mín }}^{D-2}-p_{c, \text { máx }}^{D-2}},
$$

Nótese que de las expresiones (5.8) se desprende que $S_{e, w}+S_{e, a}=1$ para todo valor de la presión efectiva $p_{c}^{e f}$.

A modo de ilustración la Fig. 5.3 muestra las curvas de saturación efectiva que se obtienen a partir de (5.8) considerando $p_{c \text {,mín }}=10 \mathrm{~Pa}, p_{c \text {,máx }}=1000 \mathrm{~Pa}$ y $D=1.5$. Como puede observarse, la saturación efectiva del agua toma el valor máximo $S_{e, w}=1$

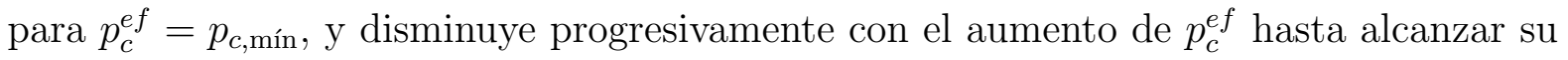
valor mínimo $S_{e, w}=0$ en $p_{c}^{e f}=p_{c \text {,máx }}$. Por su parte, como era de esperar, la saturación efectiva del aire verifica el comportamiento inverso.

Dado que el flujo bifásico suele describirse en términos de la saturación efectiva de agua, seguiremos el criterio adoptado en el Capítulo 2 de utilizar el término saturación efectiva $S_{e}$ para hacer referencia a la saturación efectiva de agua.

\subsubsection{Permeabilidades}

A la escala del REV, las presiones efectivas $p_{w}^{e f}$ y $p_{a}^{e f}$ se asumen constantes. Luego, los gradientes de presión efectivos son nulos y las fases fluirán únicamente por gravedad. Suponiendo por simplicidad que la dirección de flujo es vertical (en la dirección de la 


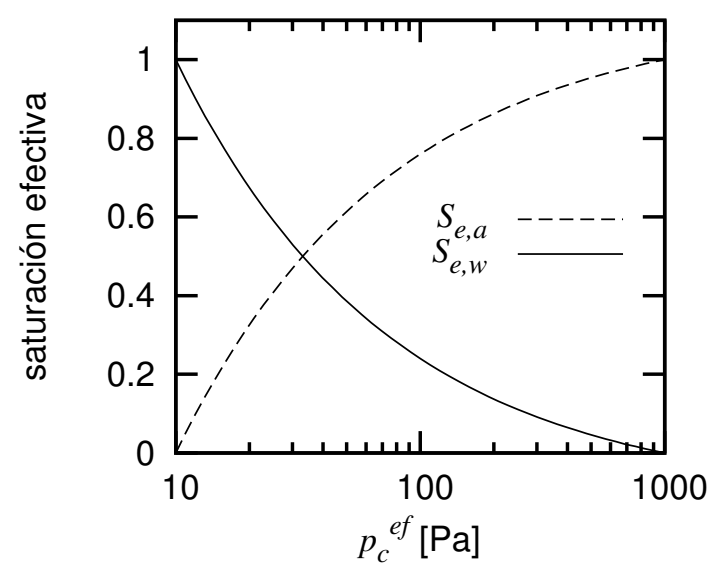

Figura 5.3: Curvas de saturación efectiva de agua $S_{e, w}$ y de aire $S_{e, a}$ en función de la presión capilar efectiva $p_{c}^{e f}$. Para el ejemplo se ha considerado $p_{c \text {,mín }}=10 \mathrm{~Pa}$, $p_{c, \text { máx }}=1000$ Pa y $D=1.5$.

gravedad), la velocidad de flujo en una fractura individual de apertura $x$ puede obtenerse resolviendo la ecuación de Navier-Stokes [7]:

$$
u_{\alpha}(x)=-\frac{x^{2}}{12 \mu_{\alpha}} \frac{\partial}{\partial z}\left(p_{\alpha}^{e f}+\rho_{\alpha} g z\right)=-\frac{\rho_{\alpha} g x^{2}}{12 \mu_{\alpha}} . \quad \alpha=a, w,
$$

donde $\alpha=a, w$ denotan al aire y al agua, respectivamente, $z$ es la coordenada vertical, $\mu_{\alpha}$ y $\rho_{\alpha}$ son la viscosidad y la densidad de la fase $\alpha$, respectivamente. La ecuación (5.9) es la conocida ley de Hagen-Poiseuille para la velocidad del flujo de un fluido entre dos placas planas paralelas separadas una distancia $x$.

El caudal total de agua $Q_{w}$ a través de una sección transversal horizontal del REV puede obtenerse integrando las velocidades de flujo individuales $u_{w}$ sobre el área ocupada por las fracturas que se encuentran saturadas por agua:

$$
Q_{w}\left(x^{e f}\right)=\int_{x_{\min }}^{x^{e f}} u_{w}(x) d A .
$$

Reemplazando las ecuaciones (5.4) y (5.9) en (5.10), obtenemos la siguiente expresión para el flujo total de agua en el REV:

$$
Q_{w}\left(x^{e f}\right)=\frac{\rho_{w} g a^{D}}{12 \mu_{w}} \frac{D-2}{D-4}\left[\left(x^{e f}\right)^{4-D}-x_{\operatorname{mín}}^{4-D}\right] .
$$

La ecuación (5.11) puede ser expresada en términos de la presión capilar efectiva usando 
la ecuación (5.5):

$$
Q_{w}\left(p_{c}^{e f}\right)=\frac{\rho_{w} g a^{D}}{12 \mu_{w}} \frac{D-2}{D-4}(2 \sigma \cos (\beta))^{4-D}\left[\left(p_{c}^{e f}\right)^{D-4}-p_{c, \text { máx }}^{D-4}\right] .
$$

Por otra parte, el caudal total de agua a la escala del REV puede expresarse a partir de la ley de Buckingham-Darcy [14]:

$$
Q_{w}\left(p_{c}^{e f}\right)=\frac{\kappa_{w}\left(p_{c}^{e f}\right) a^{2}}{\mu_{w}} \frac{\partial}{\partial z}\left(p_{w}^{e f}+\rho_{w} g z\right)=\frac{\kappa_{w}\left(p_{c}^{e f}\right) \rho_{w} g a^{2}}{\mu_{w}}
$$

donde $\kappa_{w}$ es la permeabilidad del agua y $a^{2}$ representa el área de un corte transversal del REV.

Finalmente, combinando las ecuaciones (5.12) y (5.13) se obtiene la siguiente expresión para la permeabilidad del agua:

$$
\kappa_{w}\left(p_{c}^{e f}\right)=\frac{a^{D-2}}{12} \frac{D-2}{D-4}(2 \sigma \cos (\beta))^{4-D}\left[\left(p_{c}^{e f}\right)^{D-4}-p_{c, \text { máx }}^{D-4}\right] .
$$

La expresión para la permeabilidad del aire en función de la presión capilar efectiva puede derivarse siguiendo el mismo razonamiento considerando el rango de aperturas de las fracturas ocupadas por aire $\left(x^{e f} \leq x \leq x_{\text {máx }}\right)$. En este caso la expresión de la permeabilidad del aire toma la forma:

$$
\kappa_{a}\left(p_{c}^{e f}\right)=\frac{a^{D-2}}{12} \frac{D-2}{D-4}(2 \sigma \cos (\beta))^{4-D}\left[p_{c, \text { mín }}^{D-4}-\left(p_{c}^{e f}\right)^{D-4}\right]
$$

La permeabilidad intrínseca $\kappa$ puede obtenerse tanto a partir de la ecuación (5.14) cuando $p_{c}^{e f}=p_{c \text {,mín }}(\mathrm{REV}$ completamente saturado de agua) o a partir de la ecuación (5.15) cuando $p_{c}^{e f}=p_{c, \text { máx }}(\mathrm{REV}$ completamente saturado de aire):

$$
\kappa=\frac{a^{D-2}}{12} \frac{D-2}{D-4}(2 \sigma \cos (\beta))^{4-D}\left[p_{c, \text { mín }}^{D-4}-p_{c, \text { máx }}^{D-4}\right] .
$$

Las permeabilidades relativas de agua y aire, $\kappa_{r, w} \mathrm{y} \kappa_{r, a}$, se definen como el cociente entre las ecuaciones (5.14) y (5.16) y las ecuaciones (5.15) y (5.16) respectivamente:

$$
\begin{gathered}
\kappa_{r, w}\left(p_{c}^{e f}\right)=\frac{\kappa_{w}\left(p_{c}^{e f}\right)}{\kappa}=\frac{\left(p_{c}^{e f}\right)^{D-4}-p_{c, \text { máx }}^{D-4}}{p_{c, \text { mín }}^{D-4}-p_{c, \text { máx }}^{D-4}} \\
\kappa_{r, a}\left(p_{c}^{e f}\right)=\frac{\kappa_{a}\left(p_{c}^{e f}\right)}{\kappa}=\frac{p_{c, \text { mín }}^{D-4}-\left(p_{c}^{e f}\right)^{D-4}}{p_{c, \text { mín }}^{D-4}-p_{c, \text { máx }}^{D-4}}
\end{gathered}
$$


Notar que las ecuaciones anteriores son válidas para $p_{c, \text { mín }} \leq p_{c}^{e f} \leq p_{c \text {,máx }}$. Para valores de $p_{c}^{e f} \leq p_{c, \text { mín }}$ todas las fracturas están saturadas con agua; luego $\kappa_{r, w}\left(p_{c}^{e f}\right)=1$ y $\kappa_{r, a}\left(p_{c}^{e f}\right)=0$. Por otro lado, todas las fracturas estarán completamente saturadas de aire para $p_{c}^{e f} \geq p_{c, \text { máx }}$, siendo en este caso $\kappa_{r, w}\left(p_{c}^{e f}\right)=0$ y $\kappa_{r, a}\left(p_{c}^{e f}\right)=1$.

Las permeabilidades relativas se expresan en general en función de la saturación efectiva de agua $S_{e}$. Reemplazando la expresión de $S_{e}$ dada por (5.8) en (5.17) y (5.18) y expresando las presiones capilares $p_{c \text {,mín }}$ y $p_{c \text {,máx }}$ en términos de las aperturas $x_{\text {máx }} \mathrm{y}$ $x_{\text {mín }}$, respectivamente, se obtienen las siguientes expresiones para $\kappa_{r, w}\left(S_{e}\right)$ y $\kappa_{r, a}\left(S_{e}\right)$ :

$$
\begin{gathered}
\kappa_{r, w}\left(S_{e}\right)=\frac{\left[\left(x_{\text {máx }}^{2-D}-x_{\text {mín }}^{2-D}\right) S_{e}+x_{\text {mín }}^{2-D}\right]^{\frac{4-D}{2-D}}-x_{\text {mín }}^{4-D}}{x_{\text {máx }}^{4-D}-x_{\text {mín }}^{4-D}}, \\
\kappa_{r, a}\left(S_{e}\right)=\frac{x_{\text {máx }}^{4-D}-\left[\left(x_{\text {máx }}^{2-D}-x_{\text {mín }}^{2-D}\right) S_{e}+x_{\text {mín }}^{2-D}\right]^{\frac{4-D}{2-D}}}{x_{\text {máx }}^{4-D}-x_{\text {mín }}^{4-D}} .
\end{gathered}
$$

Las ecuaciones (5.19) y (5.20) son expresiones analíticas cerradas, función de la saturación efectiva $S_{e}$, que dependen de la dimensión fractal y de las aperturas máxima y mínima de la red de fracturas.

Tanto $\kappa_{r, w}$ como $\kappa_{r, a}$ están definidas sobre todo el rango de variación de $S_{e}$. Sin embargo, como se ha mencionado en el Capítulo 2, tanto el flujo de agua como el de aire suelen establecerse en un rango más acotado de saturación efectiva. En el caso del agua, el rango sobre el cual puede detectarse flujo queda definido por $0<S_{e, 0, w} \leq S_{e}<1$, siendo $S_{e, 0, w}$ la saturación efectiva de emergencia para el flujo de agua. Análogamente, el rango de saturaciones efectivas sobre el cual puede detectarse flujo de aire queda establecido por $0<S_{e} \leq S_{e, 0, a}<1$, siendo $S_{e, 0, a}$ la saturación efectiva de emergencia para el flujo de aire $[98,28]$.

Con el propósito de obtener un modelo más realista, introducimos las saturaciones efectivas de emergencia, rescalando la saturación efectiva $S_{e}$ de las ecuaciones (5.19) y (5.20) de la siguiente manera [32, 28]:

$$
\begin{gathered}
\kappa_{r, w}\left(S_{e}\right)= \begin{cases}0 & 0 \leq S_{e} \leq S_{e, 0, w} \\
\frac{\left[\left(x_{\text {máx }}^{2-D}-x_{\text {mín }}^{2-D}\right) \frac{S_{e}-S_{e, 0, w}}{1-S_{e, 0, w}}+x_{\text {mín }}^{2-D}\right]^{\frac{4-D}{2-D}}-x_{\text {mín }}^{4-D}}{x_{\text {máx }}^{4-D}-x_{\text {mín }}^{4-D}} & S_{e, 0, w} \leq S_{e} \leq 1,\end{cases} \\
\kappa_{r, a}\left(S_{e}\right)= \begin{cases}\frac{x_{\text {máx }}^{4-D}-\left[\left(x_{\text {máx }}^{2-D}-x_{\text {mín }}^{2-D}\right) \frac{S_{e}}{S_{e, 0, a}}+x_{\text {mín }}^{2-D}\right]^{\frac{4-D}{2-D}}}{x_{\text {máx }}^{4-D}-x_{\text {mín }}^{4-D}} & 0 \leq S_{e} \leq S_{e, 0, a} \\
0 & S_{e, 0, a} \leq S_{e} \leq 1 .\end{cases}
\end{gathered}
$$


Nótese que ahora $\kappa_{r, w}$ varía de 0 a 1 para el rango de saturaciones efectivas $S_{e, 0, w} \leq S_{e} \leq$ 1 , mientras que $\kappa_{r, a}$ varía de 1 a 0 en el rango $0 \leq S_{e} \leq S_{e, 0, a}$.

Las permeabilidades relativas dadas por las ecuaciones (5.21) y (5.22) junto con las curvas de saturación efectiva dadas por (5.8) conforman un modelo constitutivo válido para la descripción del flujo bifásico inmiscible en rocas fracturadas. El modelo depende de 5 parámetros independientes $\left(D, x_{\text {mín }}, x_{\text {máx }}, S_{e, 0, w}\right.$ y $\left.S_{e, 0, a}\right)$ que poseen significado físico y geométrico.

Los parámetros $D, x_{\text {mín }}$ y $x_{\text {máx }}$ definen la geometría de la red de fracturas, por lo cual resulta interesante analizar la influencia de los mismos sobre las permeabilidades relativas. Este análisis nos permitirá inferir propiedades del medio a partir de las características de las curvas de $\kappa_{r, w}\left(S_{e}\right)$ y $\kappa_{r, a}\left(S_{e}\right)$.

La Fig. 5.4 muestra la influencia de la dimensión fractal $D$ (izquierda) y del rango de apertura de las fracturas $\left(x_{\text {mín }} / x_{\text {máx }}\right)$ (derecha) sobre la curva de permeabilidad relativa del agua $\kappa_{r, w}$. En ambos gráficos la saturación efectiva de emergencia para el flujo de agua se asume $S_{e, 0, w}=0.1$.
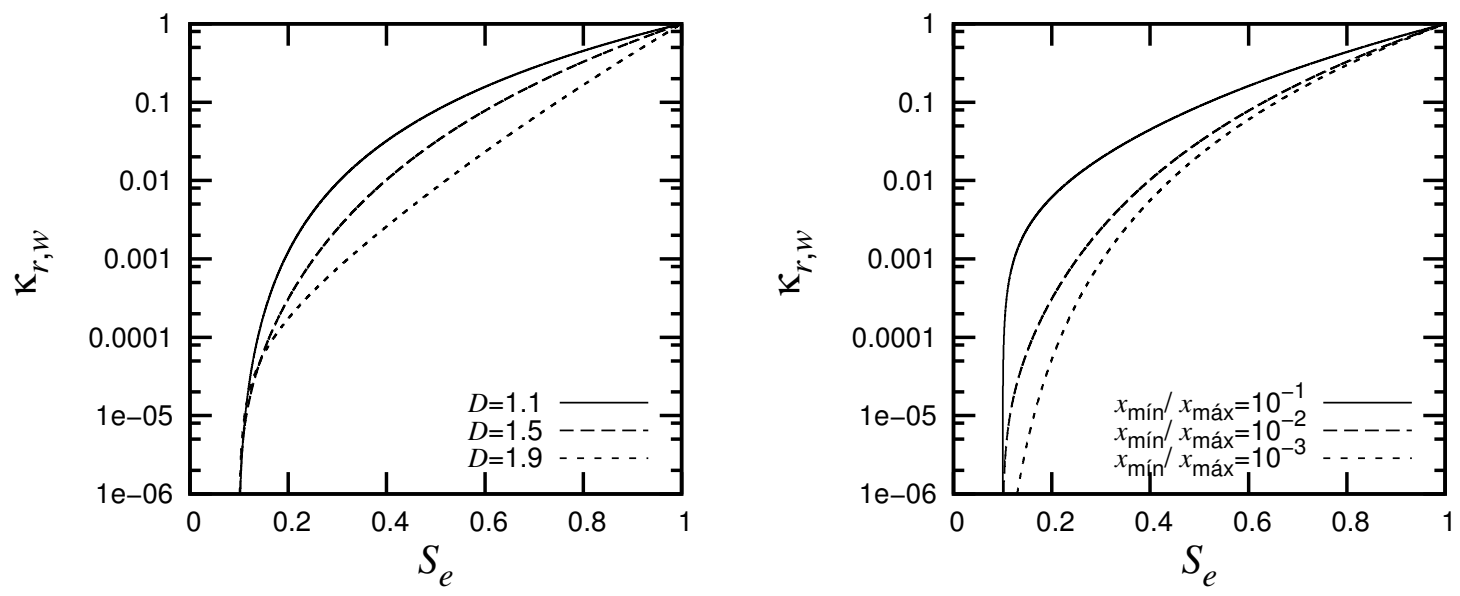

Figura 5.4: Curvas de permeabilidad relativa de agua para tres valores diferentes de dimensión fractal y $x_{\text {mín }} / x_{\text {máx }}=10^{-2}$ (izquierda) y para tres valores diferentes del rango de apertura de las fracturas y $D=1.5$ (derecha).

En el gráfico de la izquierda se puede observar que cuanto menor es la dimensión fractal $D$, mayor es el valor de $\kappa_{r, w}$ para todo el rango de saturación efectiva. Según el análisis paramétrico presentado en [39], valores pequeños de la dimensión fractal se corresponden con una mayor densidad de fracturas en el REV. Esto permite concluir 
que cuanto mayor es la densidad de fracturas, mayor es la permeabilidad relativa del agua. En el gráfico de la derecha se observa que los valores de $\kappa_{r, w}$ aumentan a medida que aumenta el cociente entre las aperturas $x_{\text {mín }}$ y $x_{\text {máx }}$. La influencia de $x_{\text {mín }} / x_{\text {máx }}$ es significativa para valores pequeños de saturación efectiva, donde $\kappa_{r, w}$ puede cambiar varios órdenes de magnitud.

El análisis del comportamiento de la permeabilidad relativa del agua puede extenderse al de la permeabilidad relativa del aire, puesto que la dependencia de $\kappa_{r, a}$ y $\kappa_{r, w}$ con $D$ y $x_{\text {mín }} / x_{\text {máx }}$ es similar. Se omite la inclusión de este análisis ya que el trabajo de Tesis se centra principalmente en la descripción del flujo de agua.

\subsubsection{Ajustes con datos experimentales}

El modelo constitutivo que se ha derivado en esta sección puede ser aplicado a la caracterización del flujo simultáneo de aire y agua en una roca fracturada. Sin embargo, las curvas de permeabilidad relativa de cada una de las fases pueden ser utilizadas independientemente para describir un flujo de tipo monofásico, cuando se asume que la otra fase se encuentra en reposo. Esto significa que bajo la hipótesis de Richards (aire a presión constante) la permeabilidad relativa del agua junto con la curva de saturación efectiva conforman un modelo constitutivo válido para la descripción del flujo no saturado de agua. Análogamente, asumiendo que el agua se encuentra a presión constante, la curva de permeabilidad relativa del aire, junto con la curva de saturación efectiva conforman un modelo constitutivo para la caracterización del flujo de aire en una roca fracturada.

En el trabajo [69] la curva de permeabilidad del agua del modelo propuesto fue utilizada para ajustar valores obtenidos mediante simulación numérica por Liu y Bodvarsson [59], mientras que la curva de permeabilidad del aire fue ajustada a datos experimentales medidos en rocas cristalinas fracturadas de Grimsel Test Site (Suiza) [12]. Los ajustes fueron realizados mediante un método de búsqueda exhaustiva, obteniendo el juego de parámetros que permite minimizar el error entre la curva del modelo propuesto y los datos [94].

Utilizando una técnica numérica similar a la que se ha presentado en el Capítulo 3, Liu y Bodvarsson [59] obtienen curvas de $\kappa_{r, w}\left(S_{e}\right)$ para dos muestras bidimensionales sintéticas de roca fracturada. Para generar las muestras los autores asumen que la matriz de la roca es impermeable y que la misma se encuentra densamente cubierta por una red de fracturas interconectadas. 


\begin{tabular}{|c|c|c|}
\hline Parámetro & Liu y Bodvarsson & Grimsel Test Site \\
\hline$D$ & 1.422 & 1.020 \\
\hline$x_{\text {mín }}[\mathrm{cm}]$ & $0.1249 \times 10^{-4}$ & $0.4701 \times 10^{-3}$ \\
\hline$x_{\text {máx }}[\mathrm{cm}]$ & 0.1419 & $0.1556 \times 10^{-2}$ \\
\hline$S_{e, 0, w}$ & 0.082 & - \\
\hline$S_{e, 0, a}$ & - & 0.76 \\
\hline
\end{tabular}

Tabla 5.1: Parámetros obtenidos mediante el ajuste de las curvas de permeabilidad relativa a los valores simulados por Liu y Bodvarsson y los datos medidos en Grimsel Test Site.

Los parámetros obtenidos a partir del ajuste del modelo propuesto (ecuación (5.21)) a los datos simulados por Liu y Bodvarsson para una de las muestras se listan en la Tabla 5.1. Notar que el rango de aperturas dado por el cociente $x_{\text {mín }} / x_{\text {máx }}$ es relativamente pequeño $\left(\simeq 10^{-4}\right)$. Según el análisis presentado en la sección anterior, es de esperar que la curva de $\kappa_{r, w}\left(S_{e}\right)$ tome valores muy pequeños para valores pequeños de $S_{e}$.

La Fig. 5.5 muestra la curva de $\kappa_{r, w}\left(S_{e}\right)$ que resulta del ajuste, junto los datos simulados por Liu y Bodvarsson. Como se evidencia en la figura el modelo propuesto logra una excelente representación de los datos simulados. Obsérvese que la permebilidad relativa varía en ocho órdenes de magnitud, detectándose la máxima tasa de disminución en la zona de saturaciones intermeadias a bajas.

Para realizar el ajuste de la curva de $\kappa_{r, a}\left(S_{e}\right)$ se utilizaron los datos experimentales obtenidos por investigadores del Swiss Federal Institute of Tecnology (Suiza) [12]. Estos datos son prácticamente los únicos disponibles en la literatura para rocas fracturadas por lo que son de suma importancia para la validación del modelo. Utilizando un dispositivo especialmente diseñado por Fischer et al. [32], se lograron determinar valores de permeabilidad relativa del aire para ocho muestras de roca fracturada extraídas de Grimsel Test Site (Suiza). Las mediciones de permebilidad se basan en un experimento de drenaje, partiendo de muestras completamente saturadas de agua, y disminuyendo progresivamente la saturación mediante evaporación.

Los parámetros obtenidos mediante el ajuste del modelo propuesto (ecuación (5.22)) 


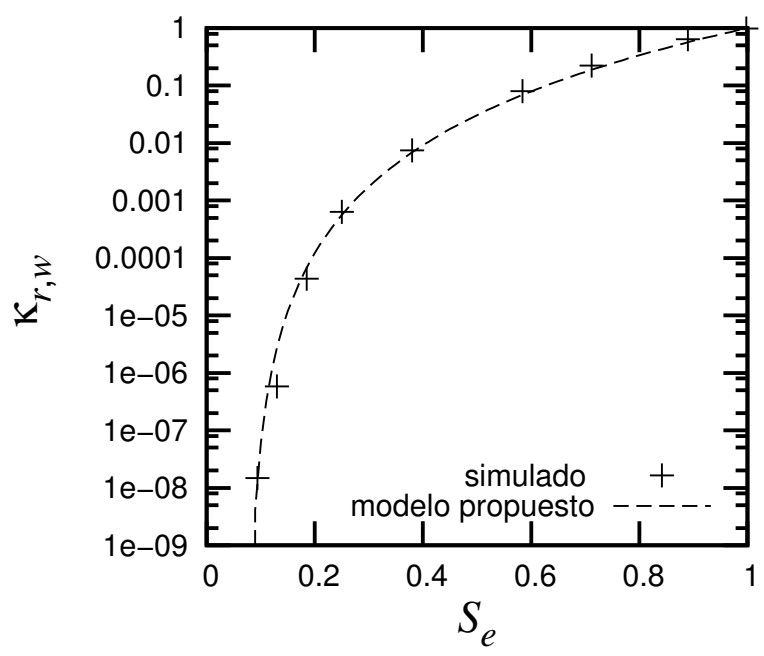

Figura 5.5: Comparación entre los valores de $\kappa_{r, w}\left(S_{e}\right)$ simulados por Liu y Bodvarsson [59] y los valores obtenidos mediante el ajuste de la ecuación (5.21).

a los datos de Grimsel Test Site se muestran en la Tabla 5.1. Nótese que la dimensión fractal $D$ es aproximadamente igual a 1, indicando el alto grado de fracturación de las muestras de roca. Otra característica del ajuste es que el valor de saturación efectiva de emergencia para el flujo de aire $S_{e, 0, a}$ es relativamente bajo. Este hecho puede atribuirse a una pobre interconección entre las fracturas que se encuentran saturadas de aire para valores de $S_{e}>S_{e, 0, a}$.

La Fig. 5.6 muestra la curva de $\kappa_{r, a}\left(S_{e}\right)$ que resulta del ajuste, junto con los datos experimentales. Nótese que a pesar de que los datos muestran algo de dispersión, el ajuste del modelo propuesto resulta satisfactorio para todo el rango de variación de la saturación efectiva.

En la sección siguiente, el modelo constitutivo propuesto será utilizado en el caso particular del flujo no saturado de agua bajo la hipótesis de Richards.

\subsection{Modelo constitutivo para el flujo no saturado de agua}

Para analizar el caso particular del flujo no saturado de agua bajo la hipótesis de Richards, debemos asumir que el aire permanece en reposo y a presión constante. En este caso, considerando a la presión efectiva del aire como presión de referencia, la altura de presión 


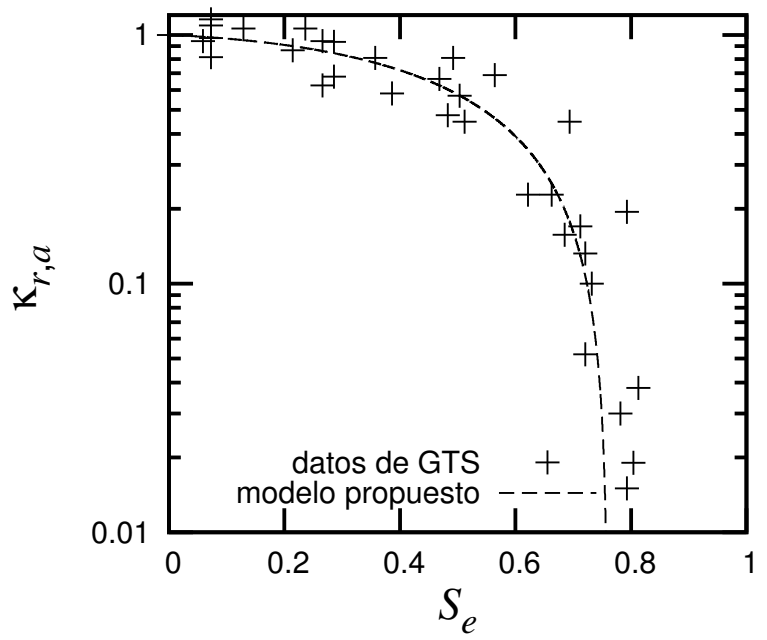

Figura 5.6: Comparación entre los valores de $\kappa_{r, a}\left(S_{e}\right)$ medidos en Grimsel Test Site (GTS) y los valores obtenidos mediante el ajuste de la ecuación (5.22).

efectiva $h^{e f}$ puede obtenerse a partir de $p_{c}^{e f}$ como:

$$
h^{e f}=\frac{p_{c}^{e f}}{\rho g} .
$$

Utilizando la ecuación (5.5) es posible vincular $h^{e f}$ con una apertura efectiva $x^{e f}$ del siguiente modo:

$$
h^{e f}=\frac{2 \sigma \cos (\beta)}{\rho g x^{e f}} .
$$

Por su definición, la permeabilidad relativa del agua $\kappa_{r, w}$ coincide con la conductividad hidráulica relativa $K_{r}$. Luego, a partir de (5.8) y (5.21) las relaciones constitutivas para el flujo no saturado podrán expresarse como:

$$
\begin{gathered}
S_{e}\left(h^{e f}\right)=\frac{h_{\text {máx }}^{D-2}-\left(h^{e f}\right)^{D-2}}{h_{\text {máx }}^{D-2}-h_{\text {mín }}^{D-2},} \\
K_{r}\left(S_{e}\right)= \begin{cases}0 & 0 \leq S_{e} \leq S_{e, 0, w} \\
\frac{\left[\left(h_{\text {min }}^{D-2}-h_{\text {máx }}^{D-2}\right) \frac{S_{e}-S_{e, 0, w}}{1-S_{e, 0, w}}+h_{\text {máx }}^{D-2}\right]^{\frac{4-D}{2-D}}-h_{\text {máx }}^{D-4}}{h_{\text {mín }}^{D-4}-h_{\text {máx }}^{D-4}} & S_{e, 0, w} \leq S_{e} \leq 1,\end{cases}
\end{gathered}
$$

siendo $h_{\text {mín }}=2 \sigma \cos (\beta) / \rho g x_{\text {máx }} \mathrm{y} h_{\text {máx }}=2 \sigma \cos (\beta) / \rho g x_{\text {mín }}$.

Las ecuaciones (5.25) y (5.26) conforman un modelo constitutivo para la caracterización del flujo no saturado en rocas fracturadas. Como puede observarse el modelo 
depende de 4 parámetros independientes: $D, h_{\text {mín }}, h_{\text {máx }}$ y $S_{e, 0, w}$. Tanto $h_{\text {mín }}$ como $h_{\text {máx }}$ pueden vincularse con las aperturas $x_{\text {mín }}$ y $x_{\text {máx }}$ si se asumen conocidos los valores de $\sigma$, $\beta, \rho \mathrm{y} g$.

El modelo constitutivo dado por las ecuaciones (5.25) y (5.26) fue derivado en el trabajo [66]. En la sección siguiente, el modelo será validado mediante su comparación con el modelo clásico de Burdine y mediante su ajuste con las relaciones constitutivas simuladas en los Capítulos 3 y 4.

\subsection{Validación del modelo}

Como se ha mencionado en el Capítulo 2, las expresiones de la conductividad hidráulica se obtienen generalmente a partir de la curva de saturación utilizando los modelos predictivos de Burdine o Mualem. En particular el modelo de Burdine ha sido utilizado por distintos autores para obtener expresiones de $K_{r}$ para rocas fracturadas [59, 39, 42].

La fórmula predictiva de Burdine puede expresarse en términos de $S_{e}$ del siguiente modo (ver ecuación (2.45)):

$$
K_{r}\left(S_{e}^{\prime}\right)=T\left(S_{e}^{\prime}\right) \frac{\int_{0}^{S_{e}^{\prime}}\left(h^{e f}\right)^{-2} d S_{e}}{\int_{0}^{1}\left(h^{e f}\right)^{-2} d S_{e}}
$$

donde $T\left(S_{e}^{\prime}\right)=\left(S_{e}^{\prime}\right)^{2}$ es el factor de tortuosidad, cuyos valores oscilan entre 0 y 1 . Cuando $T=1$ la trayectoria del flujo de agua entre dos puntos del medio poroso es recta, mientras que si $T=0$ la trayectoria entre dichos puntos es de longitud infinita.

Utilizando la curva de saturación efectiva (5.25) en (5.27) e integrando, se obtiene la siguiente expresión para la conductividad hidráulica relativa:

$$
K_{r}\left(S_{e}\right)=T\left(S_{e}\right) \frac{\left[\left(h_{\text {mín }}^{D-2}-h_{\text {máx }}^{D-2}\right) S_{e}+h_{\text {máx }}^{D-2}\right]^{\frac{4-D}{2-D}}-h_{\text {máx }}^{D-4}}{h_{\text {mín }}^{D-4}-h_{\text {máx }}^{D-4}}
$$

Nótese que si se asume $S_{e, 0, w}=0$ en (5.26), las expresiones para la conductividad hidráulica relativa (5.26) y (5.28) solo difieren en el factor de tortuosidad $T$. En el modelo propuesto el flujo de agua se produce a lo largo de trayectorias rectas definidas por las fracturas, por lo cual $T=1$ para todo el rango de saturación efectiva. Para este valor de $T$, el modelo de Burdine predice exactamente la expresión de la conductividad hidráulica relativa (5.26) cuando $S_{e, 0, w}=0$. Resulta importante destacar que el modelo de Burdine fue derivado para medios sedimentarios, donde las trayectorias seguidas por 


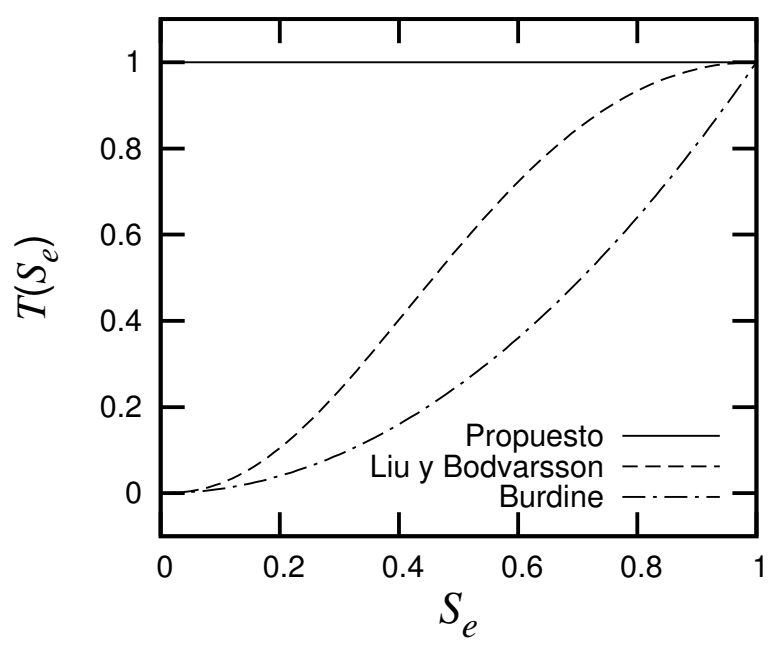

Figura 5.7: Factor de tortuosidad $T$ como función de $S_{e}$ para los modelos de Burdine, de Liu y Bodvarsson y del modelo propuesto.

las partículas de agua no son rectilíneas y dependen fuertemente del grado de saturación. Sin embargo la comparación del modelo propuesto con el modelo de Burdine nos permite extender la validez de este último al caso de rocas fracturadas utilizando un factor de tortuosidad apropiado.

La modificación del factor de tortuosidad del modelo de Burdine fue propuesta originalmente por Liu y Bodvarsson [59] para estimar la conductividad hidráulica en un medio fracturado con baja conectividad entre las fracturas. En la Fig. 5.7 se ilustra el factor de tortuosidad propuesto por Liu y Bodvarsson [59] junto con los correspondientes a los modelos de Burdine y el modelo propuesto.

El modelo que se ha propuesto representa el caso ideal en que la trayectoria del flujo de agua es rectilínea y no es modificada por la geometría del medio ni por el grado de saturación. El factor de tortuosidad de Liu y Bodvarsson [59] corresponde a un caso intermedio entre un medio fracturado sin tortuosidad $(T=1)$ y un medio sedimentario homogéneo $\left(T=S_{e}^{2}\right)$.

Como ya se ha mencionado, la determinación experimental de las relaciones constitutivas para rocas fracturadas presenta numerosas dificultades técnicas, por lo que los datos disponibles para la validación del modelo son prácticamente inexistentes [59, 103]. Es por ello que el modelo constitutivo propuesto será validado a partir del ajuste con las 
relaciones constitutivas simuladas en los Capítulos 3 y 4.

El ajuste consiste en estimar un juego de parámetros $D, h_{\operatorname{mín}}, h_{\text {máx }}$ y $S_{e, 0, w}$ que minimice el error entre las curvas de $S_{e}\left(h^{e f}\right)$ y $K_{r}\left(S_{e}\right)$ del modelo propuesto y los obtenidos mediante simulación numérica. Para realizar el ajuste se utilizó el mismo método de búsqueda exhaustiva empleado en la sección (5.3) [94]. En este punto resulta importante remarcar que si bien los modelos conceptuales empleados para la derivación analítica y la numérica son diferentes, en ambos casos se pretende caracterizar el flujo en una roca fracturada.
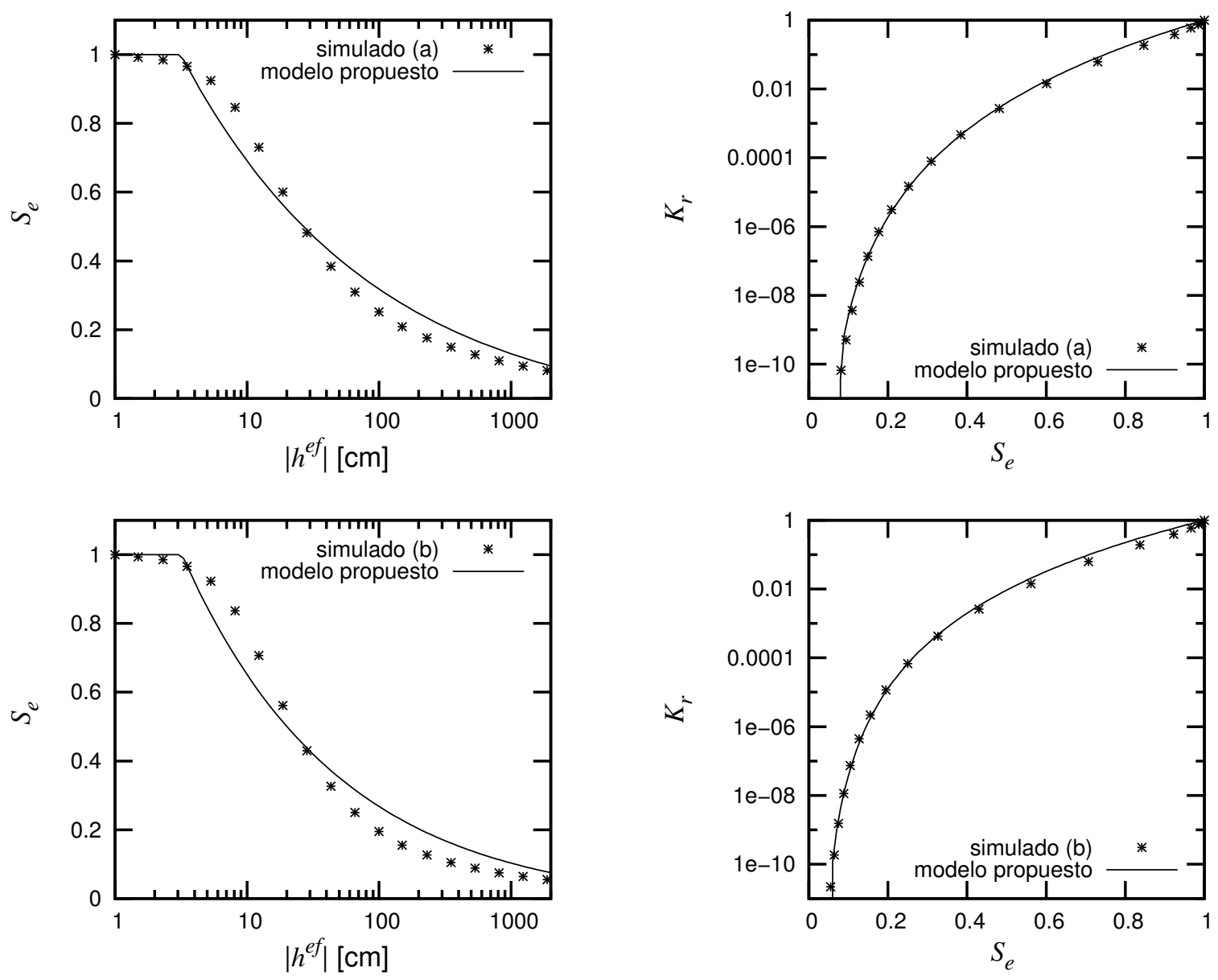

Figura 5.8: Curvas de $S_{e}\left(h^{e f}\right)$ (izquierda) y $K_{r}\left(S_{e}\right)$ (derecha) ajustadas con los valores simulados para las muestras bidimensionales generadas en el Capítulo 3. Los gráficos superiores corresponden a la muestra (a) y los inferiores a la muestra (b).

La Fig.5.8 muestra las curvas de saturación efectiva y de conductividad hidráulica relativa resultantes del ajuste con los valores simulados para las muestras bidimensionales 

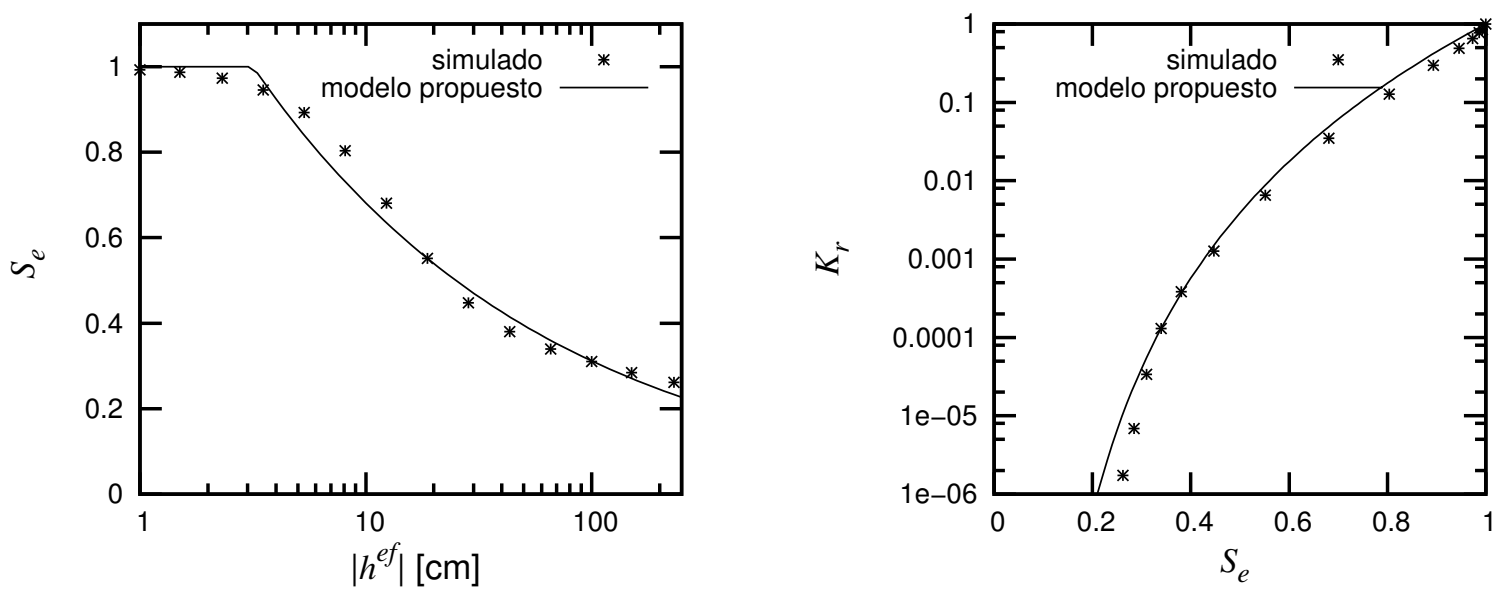

Figura 5.9: Curvas de $S_{e}\left(h^{e f}\right)$ (izquierda) y $K_{r}\left(S_{e}\right)$ (derecha) ajustadas con los valores simulados para la muestra tridimensional generada en el Capítulo 4.

de roca fracturada presentadas en el Capítulo 3. Las curvas superiores corresponden a la muestra (a) y las inferiores a la muestra (b) (ver Fig. 3.3). En la Fig. 5.9 se muestran las curvas correspondientes al ajuste obtenido para la muestra tridimensional generada en el Capítulo 4 (ver Fig. 4.3). Los parámetros ajustados para estos tres ejemplos se listan en la Tabla 5.2.

\begin{tabular}{|c|c|c|c|}
\hline \multirow{2}{*}{ Parámetro } & \multicolumn{2}{|c|}{ muestras 2D } & \multirow{2}{*}{ muestra 3D } \\
\cline { 2 - 3 } & $(\mathrm{a})$ & $(\mathrm{b})$ & \\
\hline$D$ & 1.702 & 1.632 & 1.672 \\
\hline$h_{\text {mín }}[\mathrm{cm}]$ & 3.149 & 3.215 & 3.171 \\
\hline$h_{\text {máx }}[\mathrm{cm}]$ & 47863 & 58038 & $1 \times 10^{6}$ \\
\hline$S_{e, 0, w}$ & 0.079 & 0.051 & 0.093 \\
\hline
\end{tabular}

Tabla 5.2: Parámetros ajustados del modelo propuesto a los valores simulados para las muestras bidimensionales (a) y (b) generadas en el Capítulo 3 y la muestra tridimensional generada en el Capítulo 4. 
Como puede observarse en las Figs. 5.8 y 5.9, resulta evidente que la saturación efectiva del modelo no logra reproducir en detalle los valores simulados. Esto se debe principalmente a que las características geométricas y físicas del modelo analítico no coinciden exactamente con las correspondientes características de las muestras de roca simuladas computacionalmente. Por ejemplo, las muestras de roca del experimento numérico poseen una matriz conductora de baja permeabilidad, mientras que en el modelo analítico la matriz es impermeable. Esta característica determina que para alturas de presión efectiva $h^{e f}$ menores que $h_{\text {mín }}$, las fracturas estén completamente saturadas $\left(S_{e}=1\right)$. Por su parte, en las muestras generadas numéricamente, tanto las fracturas como la matriz se encuentran parcialmente saturadas, resultando $S_{e}<1$. Luego, en las zonas cercanas a la saturación (valores pequeños de $h^{e f}$ ) existirá una diferencia entre la curva ajustada y los datos simulados, como puede observarse en los tres casos.

Otra diferencia importante entre el modelo numérico y el analítico radica en la distribución y características geométricas de las fracturas. En cada una de las muestras sintéticas que hemos considerado, las fracturas poseen a lo sumo dos valores de apertura y su orientación puede ser vertical u horizontal. En cambio, el modelo analítico asume que todas las fracturas son verticales y que las aperturas se encuentran en el rango $\left[x_{\text {mín }}, x_{\text {máx }}\right]$. Nótese que a pesar de esta diferencia, en el caso de las muestras bidimensionales la dimensión fractal $D$ ajustada para la muestra (a) es mayor que la correspondiente a la muestra (b) (ver Tabla 5.2). Este hecho indica que el modelo ajustado para la muestra (a) posee menor densidad de fracturas que el correspondiente a la muestra (b), lo que concuerda con las características de las redes de fractura generadas en forma numérica para ambas muestras.

A pesar de que el ajuste no resulta del todo satisfactorio para las curvas de saturación efectiva, no ocurre lo mismo en el caso de la permeabilidad hidráulica relativa. Como puede observarse, el modelo ajustado logra representar correctamente los valores simulados de $K_{r}\left(S_{e}\right)$ en todos los casos y para todo el rango de variación de la saturación efectiva. Nótese que el ajuste es excelente, sobre todo si se tiene en cuenta que los valores simulados de $K_{r}$ varían 6 órdenes de magnitud para el ejemplo 3D y 10 órdenes para los ejemplos 2D. No obstante los resultados obtenidos, resta aún evaluar la capacidad del modelo propuesto mediante su ajuste con datos experimentales, cuando estos se encuentren disponibles. 


\subsection{Conclusiones}

En este Capítulo se ha derivado un modelo constitutivo analítico válido para la caracterización del flujo bifásico inmiscible de aire y agua en rocas fracturadas. La curva de permeabilidad relativa del agua fue comparada con valores obtenidos mediante simulación numérica por Liu y Bodvarsson [59], mientras que la curva de permeabilidad relativa del aire fue comparada con datos experimentales de rocas cristalinas registrados en Grimsel Test Site (Suiza). En ambos casos se encontraron excelentes ajustes para el rango completo de saturación efectiva y sobre varios órdenes de magnitud de las permeabilidades del agua y el aire. Por último, utilizando las curvas de saturación efectiva y de permeabilidad relativa de agua se ha obtenido un modelo constitutivo válido para la descripción del flujo no saturado de agua. Este modelo ha sido validado mediante su comparación con el modelo clásico de Burdine. Finalmente el modelo fue testeado mediante su ajuste con las relaciones constitutivas simuladas en los Capítulos 3 y 4, lográndose una muy buena caracterización de los valores simulados. 


\section{Capítulo 6}

\section{Análisis de acuíferos heterogéneos por el método de marea inducida}

En este Capítulo se estudian los efectos producidos por la presencia de heterogeneidades de conductividad hidráulica sobre el flujo inducido por efectos de marea en acuíferos costeros. Asumiendo que la conductividad hidráulica aumenta linealmente con la distancia desde la línea de costa, se derivan dos nuevas soluciones analíticas (una exacta y otra aproximada) que permiten describir el flujo inducido por una marea periódica en términos de las fluctuaciones de altura piezométrica observables en pozos. El efecto de la heterogeneidad es evaluado mediante la comparación de la solución analítica exacta con la fórmula clásica de Jacob [44] válida para un acuífero homogéneo. Por último, la solución analítica exacta es utilizada para estimar la conductividad hidráulica en un caso real a partir del ajuste de las curvas de amplitud y de fase. Los contenidos del presente Capítulo forman parte de los trabajos [67] y [68].

\subsection{Introducción}

La distribución espacial de la conductividad hidráulica suele presentar heterogeneidades tanto a escala de laboratorio como a escala de campo. En los Capítulos 3 y 4 se ha analizado el efecto que produce la presencia de heterogeneidades en muestras de laboratorio, con el fin de calcular valores efectivos de las propiedades hidráulicas. En el presente Capítulo analizaremos un tipo particular de heterogeneidad de conductividad hidráulica a escala de campo que suele observarse en numerosos acuíferos costeros de origen aluvial [72].

En la mayoría de los acuíferos costeros las fluctuaciones periódicas de la marea oceánica (o de río) inducen fluctuaciones en los niveles de agua subterránea de los pozos cercanos a la costa. El estudio de este fenómeno brinda información sobre las propiedades hidráulicas del acuífero y ha dado lugar a una nueva técnica para estimar la conductividad hidráulica que constituye una alternativa a los ensayos de bombeo [46]. En líneas generales esta técnica, denominada comúnmente método de marea inducida, se basa en el 
análisis de las fluctuaciones de altura piezométrica observadas en pozos como respuesta a las fluctuaciones del nivel del mar debidas a la marea. Esta respuesta se traduce en cambios espacio-temporales de amplitud y fase que pueden ser registrados en pozos cercanos a la costa. La conductividad hidráulica puede ser estimada tanto a partir de los datos de amplitud (método de amplitud) como de los de fase (método de fase) mediante el ajuste de soluciones analíticas.

La interacción dinámica entre el agua subterránea y el agua de mar, inducida por oscilaciones de marea, ha sido extensamente analizada tanto mediante métodos analíticos como numéricos. Desde 1950 se ha presentado un gran número de soluciones analíticas con el propósito de describir esta interacción. Jacob [44] y Ferris [30] fueron los primeros en obtener una solución analítica a partir de resolver las ecuaciones clásicas de flujo en un acuífero confinado y homogéneo. La solución analítica para este caso particular es relativamente sencilla y ha sido ampliamente utilizada para la estimación de los parámetros hidráulicos en distintos acuíferos costeros [18, 27, 95, 29, 64, 102, 46].

Recientemente se han presentado soluciones analíticas más complejas para sistemas compuestos por un acuífero confinado por una capa semi-impermeable $[47,53,55,56,58$, 45, 57, 97, 52, 99, 40]. Estas soluciones analíticas permiten estudiar el efecto de procesos como el goteo y el almacenamiento sobre la respuesta inducida por marea. Sin embargo, todos estos resultados teóricos fueron obtenidos bajo la hipótesis de homogeneidad de las capas del sistema acuífero. Esta hipótesis discrepa significativamente con la realidad, dado que en general los acuíferos costeros exhiben heterogeneidad y anisotropía en sus propiedades hidráulicas [54, 101].

El estudio del efecto de la heterogeneidad sobre las fluctuaciones inducidas por marea ha sido abordado por algunos autores. Trefry [100] presentó soluciones analíticas para un acuífero de extensión finita formado por un número arbitrario de zonas homogéneas contíguas, sujetas a condiciones de borde lineales sinusoidales. Guo et al. [43] derivaron una solución analítica para un acuífero semi-infinito compuesto por dos zonas homogéneas diferentes. Chuang et al. [23] extendieron este modelo incluyendo el efecto de goteo y dividiendo el acuífero en un número finito de regiones horizontales homogéneas.

Para el conocimiento del autor de esta Tesis, no existen soluciones analíticas que consideren una variación continua de las propiedades hidráulicas con la distancia. En particular, una solución analítica que considere un incremento continuo de la conductividad hidráulica puede resultar de gran utilidad en el estudio de acuíferos costeros de origen 
aluvial. En estos acuíferos los sedimentos más finos se depositan aguas abajo de la zona deposicional (cerca de la línea de costa), dando como resultado un aumento progresivo de la conductividad hidráulica con la distancia a la línea de costa $[34,60,17,16,23]$. En algunos casos, la tasa de incremento de la conductividad hidráulica puede ser significativa. Montalto et al. [72] reportaron variaciones lineales de aproximadamente dos órdenes de magnitud a lo largo de transectas de 50 m en un estuario del Río Hudson, EEUU. Carol et al. [17] han inferido variaciones similares en un acuífero aluvial costero del Río Ajó, al sur de la Bahía de Samborombón, Argentina. En ambos casos, el modelo clásico de Jacob no logra predecir las fluctuaciones de altura piezométrica observadas en pozos cercanos a la costa. La razón principal de las discrepancias se atribuye a que el modelo no contempla heterogeneidades en la conductividad hidráulica.

El objetivo de este Capítulo es presentar la derivación de soluciones analíticas para las fluctuaciones de altura piezométrica inducidas por marea en un acuífero costero confinado cuya conductividad hidráulica aumenta linealmente con la distancia a la línea de costa. Se comenzará por introducir los conceptos y leyes físicas que gobiernan el flujo de agua subterránea en un acuífero confinado. Luego se presentará el modelo matemático y se establecerán las hipótesis para la descripción del proceso, lo cual nos llevará a plantear un problema con condiciones de contorno. Este problema será resuelto analíticamente, obteniéndose una solución exacta y otra aproximada. A partir del diseño de ejemplos hipotéticos se analizará el efecto de la heterogeneidad sobre las fluctuaciones de la altura piezométrica comparando las respuestas obtenidas para distintos grados de heterogeneidad. Se verificará que la solución analítica exacta converge a la solución clásica de Jacob cuando la tasa de variación de la conductividad hidráulica tiende a cero. Por último, la solución analítica exacta será utilizada para estimar la conductividad hidráulica para un caso real a partir del ajuste de las curvas de amplitud y de fase a los datos de altura piezométrica observados.

\subsection{Ecuación de flujo horizontal para un acuífero confinado}

En términos generales se define al acuífero como una formación geológica, o un estrato, que (a) contiene agua en su espacio poral y (b) permite el flujo de enormes cantidades de agua bajo condiciones naturales. En la mayoría de los casos un acuífero yace sobre una formación impermeable, la cual impide que el agua migre a profundidad. Si además 
el acuífero está limitado por un techo impermeable se dice que el acuífero es confinado, de lo contrario es no confinado. En este Capítulo trataremos con acuíferos confinados, aunque muchos de los resultados que se obtienen también son válidos para acuíferos no confinados cuando se satisfacen ciertas hipótesis.

El flujo de agua en un acuífero es esencialmente horizontal, o bien, puede aproximarse como tal. Esta aproximación es exacta en acuíferos horizontales, homogéneos e isótropos, y sigue siendo válida cuando el espesor vertical del acuífero es variable, siempre y cuando las variaciones sean mucho menores que el espesor medio del acuífero. La aproximación de flujo horizontal falla en regiones donde el flujo tiene una componente vertical significativa, por ejemplo, en las cercanías de un pozo de bombeo que no atraviesa el espesor completo del acuífero.

Otra de las características del flujo de agua en un acuífero es que las variaciones en la densidad del agua suelen ser despreciables. Asumiremos entonces que el agua en el interior del acuífero es un fluido homogéneo de densidad constante $\rho$.

En la sección siguiente introduciremos el concepto de almacenamiento que interviene en las ecuaciones que describen el flujo de agua en un medio poroso completamente saturado.

\subsubsection{Almacenamiento}

Cuando se perfora un pozo que atraviesa un acuífero confinado, el nivel de agua en el pozo suele ascender por encima de la base del techo impermeable, pudiendo incluso alcanzar la superficie (ver Fig. 6.1 (a)). Esto se debe a que la presión del agua que satura un acuífero confinado puede ser considerablemente elevada. La altura de la columna de agua en un pozo de observación apropiadamente construido indicaría la altura piezométrica en el punto donde el pozo está en contacto con el acuífero.

Si se considera un volumen de control en el interior de un acuífero confinado, el mismo estará sometido a esfuerzos internos debidos a la presión ejercida por el agua, y a esfuerzos externos ejercidos por la formación en la cual el volumen se encuentra inmerso. En la mayoría de los problemas de flujo subterráneo, el esfuerzo ejercido por la formación permanece inalterado, mientras que los esfuerzos internos producidos por las fluctuaciones en la presión del agua suelen registrar variaciones importantes. Estas variaciones modifican la capacidad de almacenamiento de agua del medio a causa de la 


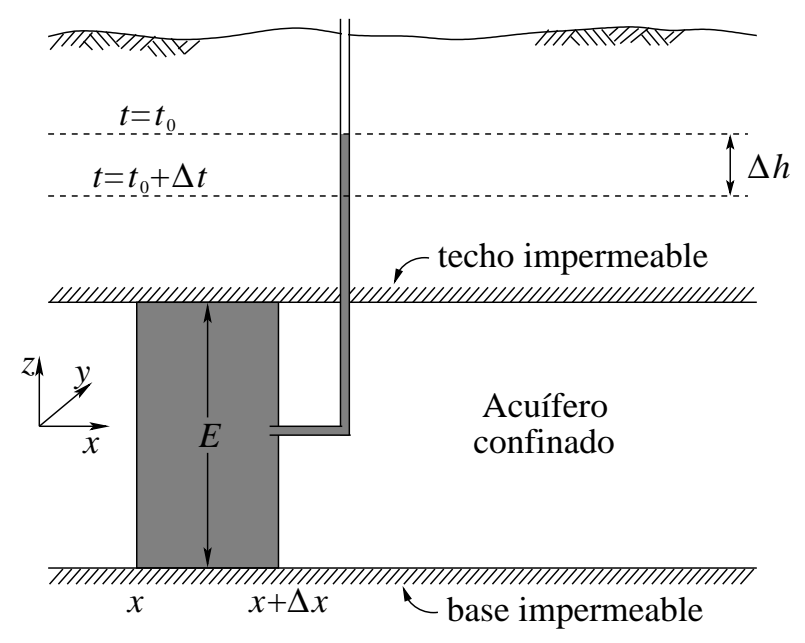

(a)

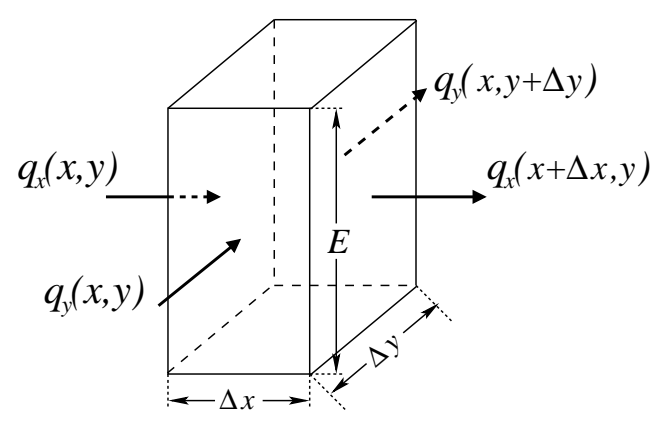

(b)

Figura 6.1: Representación esquemática del flujo en un acuífero confinado bajo la aproximación de flujo horizontal (adaptada de [7]).

deformación.

El almacenamiento S de un acuífero, también llamado coeficiente de almacenamiento, expresa la relación entre los cambios en la cantidad de agua almacenada en un acuífero y los correspondientes cambios en la altura piezométrica. Asumiendo un acuífero horizontal, el almacenamiento se define como el volumen de agua $\Delta V_{w}$ que ingresa/egresa al acuífero por unidad de área horizontal $\Delta A$ y por unidad de aumento/disminución de la altura piezométrica $\Delta h$ :

$$
\mathrm{S}=\frac{\Delta V_{w}}{\Delta A \Delta h}
$$

El almacenamiento $\mathrm{S}$ depende de las propiedades elásticas del medio poroso y del agua. El análisis de esta dependencia excede los objetivos de la Tesis; para mayor información sobre este aspecto del problema se refiere a [7].

El almacenamiento específico $\mathrm{S}_{s}$ se define como el volumen de agua que ingresa/egresa al acuífero por unidad de volumen del acuífero y por unidad de aumento/disminución de la altura piezométrica. Luego:

$$
\mathrm{S}_{s}=\frac{\Delta V_{w}}{\Delta A E \Delta h}=\frac{\mathrm{S}}{E}
$$

siendo $E$ el espesor del acuífero confinado.

Utilizando el concepto de almacenamiento que hemos introducido procederemos a derivar la ecuación que gobierna el flujo en un acuífero confinado. Esta ecuación se 
obtiene a partir de plantear el balance de masa en un volumen de control situado en el interior del acuífero.

\subsubsection{Ecuación de balance de masa}

Consideremos un volumen de control $V=E \Delta x \Delta y$ en el interior de un acuífero confinado, como se muestra en la Fig. 6.1 (b). Para derivar la ecuación de conservación se realiza un balance del contenido de agua en $V$ durante un período de tiempo $\Delta t$ arbitrariamente pequeño. Asumiendo que no existen fuentes ni sumideros en el interior de $V$, la ecuación de balance puede establecerse en términos conceptuales del siguiente modo:

$$
\left[\begin{array}{c}
\text { Volumen de agua que } \\
\text { ingresa/egresa a través } \\
\text { de las caras de } V \text { en } \Delta t
\end{array}\right]=\left[\begin{array}{c}
\text { Variación del } \\
\text { volumen de agua } \\
\text { en } V \text { durante } \Delta t
\end{array}\right] \text {. }
$$

El miembro izquierdo de la ecuación (6.3) se obtiene calculando la cantidad de agua que ingresa/egresa del volumen $V$ durante el período $\Delta t$ a través de sus caras verticales (las caras horizontales se encuentran impermeabilizadas). Asumiendo que el flujo es horizontal (ver Fig. $6.1(b))$ resulta:

$$
\left[\begin{array}{c}
\text { Volumen de agua que } \\
\text { ingresa/egresa a través } \\
\text { de las caras de } V \text { en } \Delta t
\end{array}\right]=-\Delta q_{x} E \Delta y \Delta t-\Delta q_{y} E \Delta x \Delta t
$$

donde $\Delta q_{x}=q_{x}(x+\Delta x, y)-q_{x}(x, y)$ y $\Delta q_{y}=q_{y}(x, y+\Delta y)-q_{y}(x, y)$.

El miembro derecho de la ecuación (6.3) puede expresarse en términos del almacenamiento específico $\mathrm{S}_{s}$ haciendo uso de la ecuación (6.2):

$$
\left[\begin{array}{c}
\text { Variación del } \\
\text { volumen de agua } \\
\text { en } V \text { durante } \Delta t
\end{array}\right]=\mathrm{S}_{s} E \Delta x \Delta y \Delta h
$$

Introduciendo (6.4) y (6.5) en (6.3) y dividiendo la expresión resultante por $E \Delta x \Delta y \Delta t$ se obtiene:

$$
-\left(\frac{\Delta q_{x}}{\Delta x}+\frac{\Delta q_{y}}{\Delta y}\right)=\mathrm{S}_{s} \frac{\Delta h}{\Delta t}
$$


Tomando el límite cuando $\Delta x, \Delta y, \Delta t \rightarrow 0$, la ecuación (6.6) resulta:

$$
-\nabla \cdot \mathbf{q}=\mathrm{S}_{s} \frac{\partial h}{\partial t}
$$

donde $\mathbf{q}=\left(q_{x}, q_{y}\right)$ es el vector de flujo de agua.

Finalmente, haciendo uso de la ley de Darcy, la ecuación de balance de masa resulta:

$$
\boldsymbol{\nabla} \cdot(K \nabla h)=\mathrm{S}_{s} \frac{\partial h}{\partial t}
$$

En la sección siguiente la ecuación (6.8) será utilizada para modelar las fluctuaciones de altura piezométrica inducidas por una marea periódica en un acuífero costero confinado.

\subsection{Modelo matemático y soluciones analíticas}

Para representar la interacción entre un acuífero costero y el mar utilizaremos el mismo modelo conceptual propuesto por Jacob [44]. Este modelo está esquematizado en la Fig. 6.2. Tanto el acuífero como las capas impermeables se extienden infinitamente desde la línea de costa en dirección tierra adentro. Las capas son horizontales y su frontera con el mar se asume vertical. Para la descripción matemática del problema consideraremos el eje $x$ horizontal, perpendicular a la línea de costa, positivo en sentido tierra adentro y con origen en la línea de costa. El nivel medio del mar se elige como datum (o nivel cero) de la altura piezométrica.

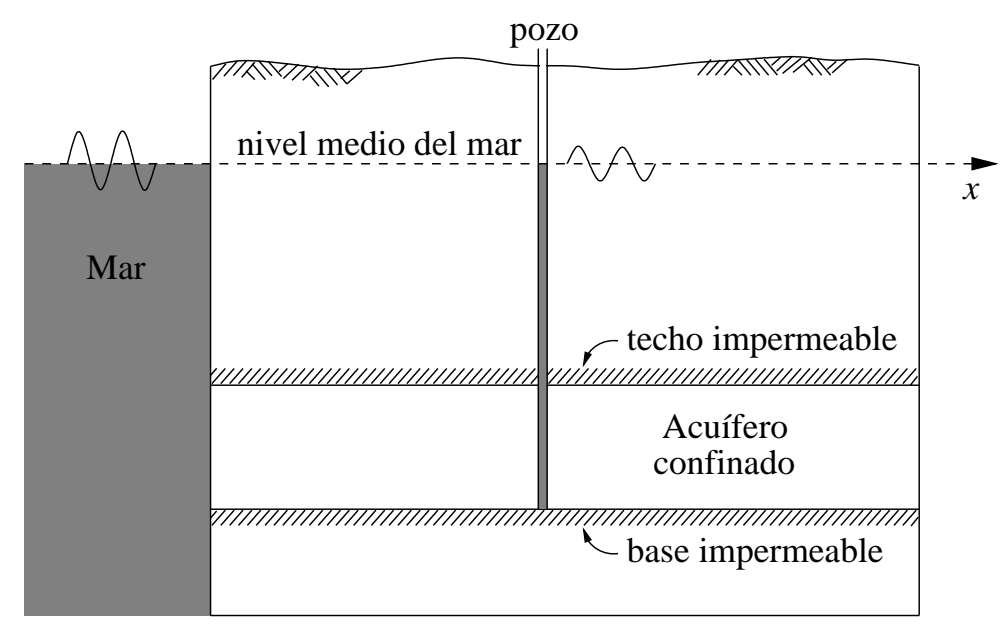

Figura 6.2: Representación esquemática de un acuífero costero confinado. El eje $x$ está representado por la línea de puntos. 
Para la descripción del flujo en este sistema supondremos que el efecto de las variaciones de densidad sobre el flujo de agua es despreciable y que el almacenamiento específico es constante (lo que es una buena aproximación en la mayoría de los casos). Bajo estas hipótesis y asumiendo una conductividad hidráulica heterogénea, la altura piezométrica $h(x, t)$ verificará la ecuación (6.8) en su versión unidimensional:

$$
\frac{\partial}{\partial x}\left(K(x) \frac{\partial h}{\partial x}\right)=\mathrm{S}_{s} \frac{\partial h}{\partial t} .
$$

La condición de borde en la interfase mar-acuífero estará dada por la oscilación de la marea, la cual se asume de tipo cosenoidal:

$$
h(0, t)=A \cos (\omega t)
$$

siendo $A$ la amplitud y $\omega$ la frecuencia angular de la marea. En el infinito, la condición de borde impuesta es:

$$
\lim _{x \rightarrow \infty} K(x) \frac{\partial h}{\partial x}=0
$$

la cual establece que el flujo debe anularse para $x \rightarrow \infty$. Finalmente, cabe señalar que solo se considerarán soluciones periódicas, por lo que $-\infty<t<\infty$ y no será necesario imponer condiciones iniciales [44].

\subsubsection{Solución analítica para un acuífero homogéneo}

En esta sección obtendremos la ecuación clásica de Jacob que utilizaremos de referencia para el análisis del efecto de las heterogeneidades.

Asumiendo un acuífero homogéneo $(K(x)=K)$ el problema de valores de contorno (6.9)-(6.11) toma la forma:

$$
\begin{gathered}
\frac{\partial^{2} h}{\partial x^{2}}=\frac{\mathrm{S}_{s}}{K} \frac{\partial h}{\partial t}, \\
h(0, t)=A \cos (\omega t), \\
\lim _{x \rightarrow \infty} \frac{\partial h}{\partial x}=0 .
\end{gathered}
$$

Para hallar la solución de (6.12)-(6.14) procedemos a resolver el siguiente problema equivalente en el campo complejo: Hallar $H(x, t)$ tal que

$$
\begin{gathered}
\frac{\partial^{2} H}{\partial x^{2}}=\frac{\mathrm{S}_{s}}{K} \frac{\partial H}{\partial t}, \\
H(0, t)=A e^{i \omega t},
\end{gathered}
$$




$$
\lim _{x \rightarrow \infty} \frac{\partial H}{\partial x}=0
$$

siendo la solución buscada $h(x, t)=\operatorname{Re}[H(x, t)]$.

La solución del problema (6.15)-(6.17) puede obtenerse mediante el método de separación de variables proponiendo

$$
H(x, t)=A X(x) e^{i \omega t}
$$

Reemplazando la expresión (6.18) en (6.15)-(6.17) se obtiene el siguiente problema de contorno en términos de $X(x)$ :

$$
\begin{gathered}
\frac{d^{2} X}{d x^{2}}-\frac{i \omega \mathrm{S}_{s}}{K} X=0 . \\
X(0)=1, \\
\lim _{x \rightarrow \infty} \frac{d X}{d x}=0 .
\end{gathered}
$$

La solución general de la ecuación (6.19) viene dada por:

$$
X(x)=C_{1} e^{-(1+i) a x}+C_{2} e^{(1+i) a x},
$$

siendo $C_{1}$ y $C_{2}$ constantes complejas, y a un parámetro real dado por:

$$
a=\sqrt{\frac{\omega \mathrm{S}_{s}}{2 K}} .
$$

Imponiendo las condiciones de borde (6.20) y (6.21) se obtiene $C_{1}=1$ y $C_{2}=0$. Luego, en virtud de (6.18) la solución del problema (6.12)-(6.14) resulta:

$$
h(x, t)=A e^{-a x} \cos (-a x+\omega t) .
$$

La ecuación (6.24) fue obtenida por Jacob [44] (e independientemente por Ferris [30]) bajo las mismas hipótesis que hemos adoptado. Comparando esta ecuación con la fluctuación de marea dada por la condición de borde (6.10) pueden observarse dos efectos: (a) un decaimiento exponencial en la amplitud de $h(x, t)$ con la distancia a la línea de costa $x$ a través del factor $e^{-a x}$ y (b) un desfasaje o retraso temporal que aumenta linealmente con $x$, dado por $-a x / \omega$. Se definen entonces el coeficiente de amplitud $c$ y la fase temporal $\tau$ como:

$$
c(x)=e^{-a x}
$$




$$
\tau(x)=-\frac{1}{\omega} a x .
$$

Notar que el retraso de fase puede definirse en términos del retraso temporal dado por (6.26) multiplicando esta expresión por $\omega$.

Como puede observarse, las expresiones de $c(x)$ y $\tau(x)$ quedan determinadas por el valor del coeficiente $a$, denominado coeficiente de propagación de marea [36]. Dado que $a^{-1}$ tiene unidades de longitud, se lo suele llamar longitud característica. Para una distancia $x=a^{-1}$ la amplitud de las fluctuaciones de altura piezométrica inducidas por marea decae a $A / e \sim 0.36 A$. En la última sección de este Capítulo veremos que las expresiones (6.25) y (6.26) proveen dos métodos (de amplitud y de fase, respectivamente) para la estimación de los parámetros hidráulicos en acuíferos costeros mediante el método de marea inducida.
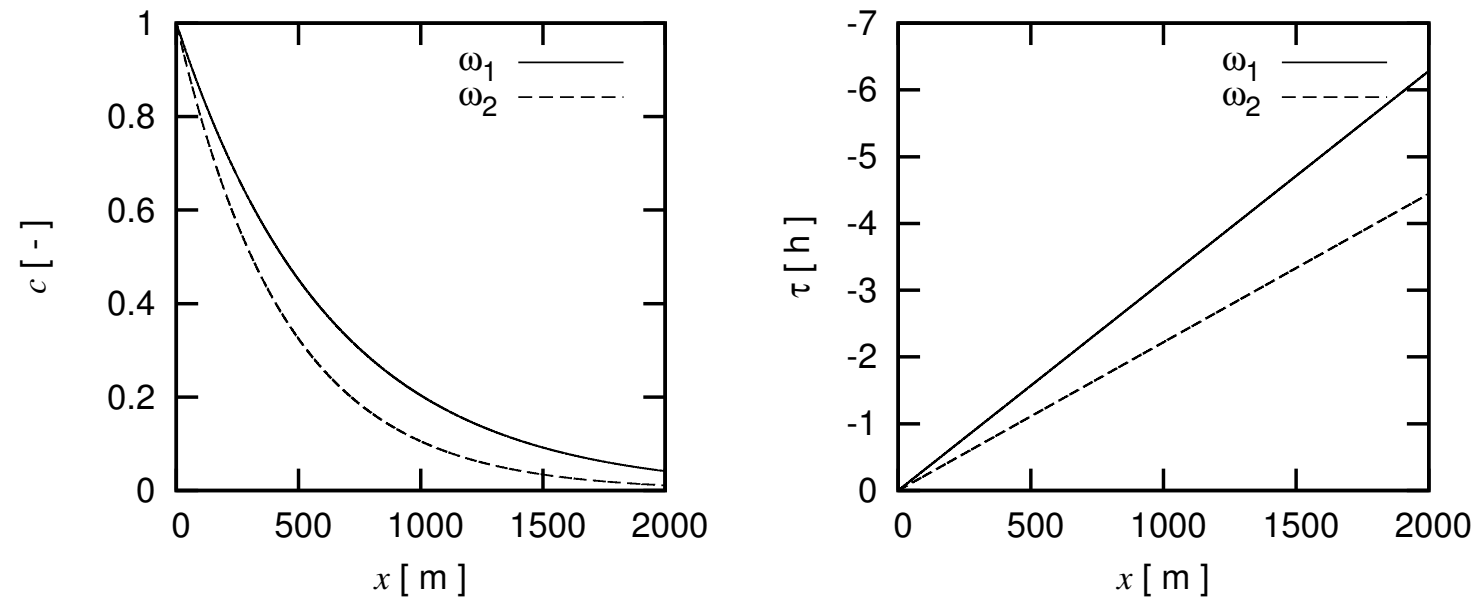

Figura 6.3: Coeficiente de amplitud $c$ (izquierda) y fase temporal $\tau$ (derecha) versus distancia $x$ para un ejemplo hipotético, considerando dos valores de frecuencia angular de marea.

A modo de ilustración la Fig. 6.3 muestra las curvas de $c(x)$ (izquierda) y $\tau(x)$ (derecha) para un ejemplo hipotético. En el ejemplo se ha supuesto $K=1 \mathrm{~m} / \mathrm{h}$, $\mathrm{S}_{s}=10^{-5} \mathrm{~m}^{-1}$, y se han calculado las curvas de $c$ y $\tau$ para dos frecuencias angulares diferentes: $\omega_{1}=0.506 \mathrm{~h}^{-1}$ (correspondiente a un período semidiurno de $12.4 \mathrm{~h}$ ) y $\omega_{2}=2 \omega_{1}$ (período de $6.2 \mathrm{~h}$ ). Nótese que para la frecuencia $\omega_{2}$ se observa una mayor caída de amplitud debido a que el coeficiente de propagación de marea es mayor en este caso. Por su parte, el retraso de fase temporal resulta menor para $\omega_{2}$, debido a que la pendiente de $\tau(x)$ dada por $-a / \omega_{2}$ es menor que la correspondiente a $\omega_{1}$. 


\subsubsection{Solución analítica exacta para un acuífero heterogéneo}

En esta sección se derivará la solución analítica exacta del problema (6.9)-(6.11) asumiendo que la conductividad hidráulica del acuífero aumenta linealmente con la distancia a la línea de costa $x$ :

$$
K(x)=K_{0}(1+b x),
$$

siendo $K_{0}$ la conductividad hidráulica del acuífero en $x=0$ y $b$ la tasa de aumento de la conductividad hidráulica $(b>0)$. En principio, un modelo lineal para $K(x)$ podría considerarse arbitrario, sin embargo es un primer paso hacia una descripción más realista para cierto tipo de acuíferos. Por ejemplo, Cardenas [16] utiliza la ecuación (6.27) para describir algunos rasgos de la dinámica del agua subterránea en una isla fluvial que no puede ser representada satisfactoriamente con una conductividad hidráulica constante. Por otra parte, la ecuación (6.27) podría resultar de utilidad en el estudio de acuíferos de origen aluvial. En estos acuíferos los sedimentos más finos se depositan aguas abajo de la zona deposicional (cerca de la línea de costa), dando como resultado un aumento progresivo de la conductividad hidráulica con la distancia a la línea de costa [34, 60, 17, 16, 23]. En algunos casos, la tasa de incremento de la conductividad hidráulica puede ser significativa como las reportadas por Montalto et al. [72] en un estuario del Río Hudson (EEUU) y por Carol et al. [17] en un acuífero aluvial costero del Río Ajó, al sur de la Bahía de Samborombón, Argentina. Cabe mencionar que la conductividad hidráulica dada por la ecuación (6.27) tiende a infinito cuando $x$ tiende a infinito. Este no es un comportamiento realista para la conductividad hidráulica; sin embargo, como se verá en la sección siguiente, la solución analítica no se ve afectada por los valores de la conductividad hidráulica muy alejados de la costa.

Adoptando el modelo lineal de $K(x)$ dado por (6.27), el problema de valores de contorno (6.9)-(6.11) puede expresarse del siguiente modo:

$$
\begin{gathered}
\frac{\partial}{\partial x}\left(K_{0}(1+b x) \frac{\partial h}{\partial x}\right)=\mathrm{S}_{s} \frac{\partial h}{\partial t}, \\
h(0, t)=A \cos (\omega t), \\
\lim _{x \rightarrow \infty} K_{0}(1+b x) \frac{\partial h}{\partial x}=0 .
\end{gathered}
$$


Procediendo en forma análoga que en el caso homogéneo, planteamos el siguiente problema equivalente en términos de $H(x, t)$ :

$$
\begin{gathered}
\frac{\partial}{\partial x}\left(K_{0}(1+b x) \frac{\partial H}{\partial x}\right)=\mathrm{S}_{s} \frac{\partial H}{\partial t}, \\
H(0, t)=A e^{i \omega t} \\
\lim _{x \rightarrow \infty} K_{0}(1+b x) \frac{\partial H}{\partial x}=0 .
\end{gathered}
$$

Nuevamente, la solución del problema (6.31)-(6.33) verifica $\operatorname{Re}[H(x, t)]=h(x, t)$.

Proponiendo $H(x, t)=A X(x) e^{i \omega t}$ y reemplazando en (6.31)-(6.33) se obtiene el siguiente problema en términos de $X(x)$ :

$$
\begin{gathered}
\frac{d}{d x}\left((1+b x) \frac{d X}{d x}\right)+b^{2} \Lambda^{2} X=0, \\
X(0)=1, \\
\lim _{x \rightarrow \infty}(1+b x) \frac{d X}{d x}=0,
\end{gathered}
$$

siendo

$$
\Lambda^{2}=-i \frac{\omega \mathrm{S}_{s}}{b^{2} K_{0}}=-i 2\left(\frac{a}{b}\right)^{2}
$$

Notar que en (6.37) hemos utilizado la definición del coeficiente de propagación de marea $a$ (ecuación (6.23)) para un acuífero homogéneo de conductividad hidráulica $K=K_{0}$.

Para hallar la solución general de (6.34) se propone el siguiente cambio de variables:

$$
u=2 \Lambda \sqrt{1+b x}
$$

siendo $\Lambda=a(-1+i) / b$. Reemplazando (6.38) en (6.34) se obtiene la siguiente ecuación para $X$ en función de $u$ :

$$
u^{2} \frac{d^{2} X}{d u^{2}}+u \frac{d X}{d u}+u^{2} X=0
$$

La ecuación diferencial ordinaria (6.39) es la ecuación de Bessel de orden cero. Su solución general puede expresarse como [1]:

$$
X(u)=C_{1} J_{0}(u)+C_{2} Y_{0}(u)
$$

donde $J_{0}$ y $Y_{0}$ son las funciones de Bessel de orden cero de primera y segunda especie, respectivamente, y $C_{1}$ y $C_{2}$ son constantes complejas. 
Utilizando expresiones asintóticas para las derivadas de $J_{0}$ y $Y_{0}$ [3], se puede demostrar que la condición de borde (6.36) se satisface cuando

$$
\left(C_{1}+i C_{2}\right)=0
$$

Luego

$$
X(u)=C_{1}\left(J_{0}(u)+i Y_{0}(u)\right)=C_{1} H_{0}^{(1)}(u)
$$

siendo $H_{0}^{(1)}$ la función de Hankel de orden cero y primera especie [1]. Por otra parte, imponiendo la condición de borde (6.35) a la ecuación (6.42) tenemos:

$$
C_{1}=\left[H_{0}^{(1)}(2 \Lambda)\right]^{-1}
$$

Reemplazando (6.43) y (6.38) en (6.42) obtenemos la solución del problema de valores de contorno (6.34)-(6.36) :

$$
X(x)=\frac{H_{0}^{(1)}(2 \Lambda \sqrt{1+b x})}{H_{0}^{(1)}(2 \Lambda)} .
$$

Finalmente, dado que $h(x, t)=\operatorname{Re}[H(x, t)]=\operatorname{Re}\left[A X(x) e^{i \omega t}\right]$, la solución del problema (6.28)-(6.30) resulta:

$$
h(x, t)=\operatorname{Re}\left[A \frac{H_{0}^{(1)}(2 \Lambda \sqrt{1+b x})}{H_{0}^{(1)}(2 \Lambda)} e^{i \omega t}\right] .
$$

Como puede observarse, la expresión de $h(x, t)$ dada por $(6.45)$ es un tanto más compleja que la solución correspondiente al caso homogéneo (6.24) y su dependencia con los parámetros del modelo no puede ser interpretada en forma inmediata por simple inspección de su expresión. En la sección siguiente se presentará un ejemplo hipotético que permitirá comprender el efecto de la heterogeneidad lineal a partir de comparar las respuestas del modelo heterogéneo con las del modelo homogéneo.

\subsection{Análisis de la solución analítica exacta}

Para explorar la influencia de la heterogeneidad de conductividad hidráulica sobre las fluctuaciones de altura piezométrica se diseñó el ejemplo hipotético que se detalla a continuación. Los parámetros hidráulicos del acuífero confinado se asumen: $K_{0}=1 \mathrm{~m} / \mathrm{h}$, $\mathrm{S}_{s}=10^{-5} \mathrm{~m}^{-1}$ y $b=10^{-2} \mathrm{~m}^{-1}$. La marea es considerada semidiurna (período de $12.4 \mathrm{~h}$ ) 
(a)

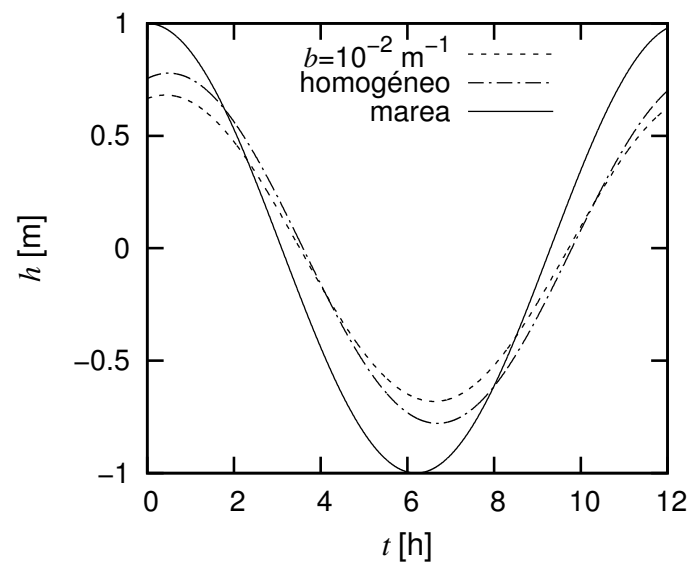

(b)

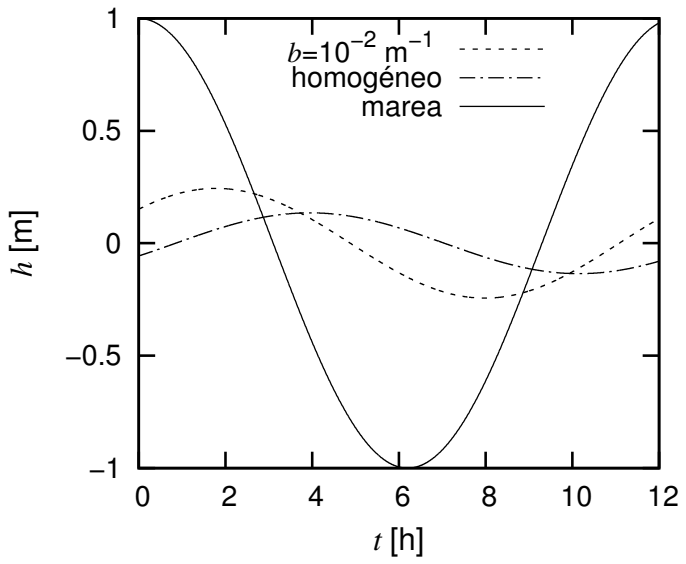

Figura 6.4: Fluctuaciones de altura piezométrica inducidas por marea en función del tiempo en dos puntos de observación: (a) $a x=0.25$ y (b) $a x=2.0$.

con una amplitud $A=1 \mathrm{~m}$. El coeficiente de propagación de marea calculado mediante la ecuación (6.23) es $a=1.59 \times 10^{-3} \mathrm{~m}^{-1}$. Por simplicidad las distancias se expresan en forma adimensional, multiplicando $x$ por $a$. El cálculo de las funciones de Hankel que intervienen en la expresión (6.45) se efectuó utilizando una subrutina Fortran de acceso libre $[113]^{1}$.

La Fig. 6.4 muestra la marea junto con las fluctuaciones de altura piezométrica tanto para el caso heterogéneo (ecuación (6.45)) como para el homogéneo asumiendo $K=K_{0}$ (ecuación (6.24)). Las series de tiempo se ilustran en dos puntos de observación, uno cercano a la línea de costa $(a x=0.25)$ y otro alejado $(a x=2.0)$. Para $a x=0.25$ las fluctuaciones inducidas en el acuífero heterogéneo tienen una amplitud menor que la correspondiente al acuífero homogéneo. Sin embargo, lejos de la costa $(a x=2)$, la amplitud de las fluctuaciones dadas por (6.45) son mayores que las del caso homogéneo, observándose además un desfasaje temporal significativo entre ambas curvas. Este ejemplo sencillo muestra que el efecto de la heterogeneidad sobre las fluctuaciones de altura piezométrica puede ser importante y tiene una fuerte dependencia con la distancia a la línea de costa.

Para un mejor entendimiento del efecto de la heterogeneidad sobre las fluctuaciones inducidas se analizarán el coeficiente de amplitud y el retraso temporal como funciones de la distancia a la línea de costa. El coeficiente de amplitud $c(x)$ y el retraso temporal

\footnotetext{
${ }^{1}$ La subrutina se encuentra disponible en http://jin.ece.illinois.edu/ .
} 
$\tau(x)$ de las fluctuaciones inducidas vienen dados por las siguientes expresiones:

$$
\begin{gathered}
c(x)=\sqrt{B_{r}^{2}+B_{i}^{2}}, \\
\tau(x)=-\frac{1}{\omega} \tan ^{-1}\left(\frac{B_{i}}{B_{r}}\right),
\end{gathered}
$$

donde:

$$
B_{r}=\operatorname{Re}\left[\frac{H_{0}^{(1)}(2 \Lambda \sqrt{1+b x})}{H_{0}^{(1)}(2 \Lambda)}\right], \quad B_{i}=\operatorname{Im}\left[\frac{H_{0}^{(1)}(2 \Lambda \sqrt{1+b x})}{H_{0}^{(1)}(2 \Lambda)}\right] .
$$

La Fig. 6.5 muestra los coeficientes de amplitud calculados a partir de la ecuación (6.46) para tres valores diferentes de la tasa de incremento de la conductividad hidráulica $b=10^{-1}, 10^{-2}, 10^{-3} \mathrm{~m}^{-1}$. Como referencia, la figura incluye la curva de $c(x)$ para un acuífero homogéneo dado por la ecuación (6.25).

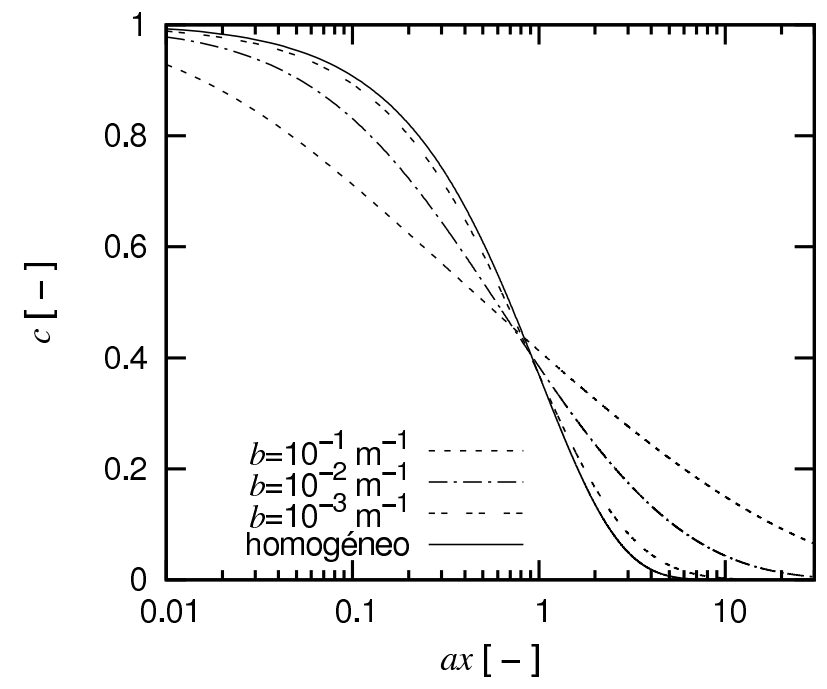

Figura 6.5: Coeficiente de amplitud $c$ versus distancia adimensional ax en escala logarítmica para diferentes tasas de incremento de la conductividad hidráulica.

En comparación con el modelo homogéneo, la heterogeneidad lineal produce amplitudes más pequeñas para distancias menores que aproximadamente $a^{-1}$ (distancia característica). En esta región cercana a la costa, el efecto de amortiguamiento de la señal se acentúa con el aumento de $b$. A medida que $x$ aumenta, el comportamiento se revierte: las amplitudes correspondientes al acuífero heterogéneo se hacen mayores, permitiendo una mayor penetración de las fluctuaciones inducidas en el acuífero. Este efecto se acentúa con el aumento de la distancia a la línea de costa, particularmente para valores grandes de $b$. 


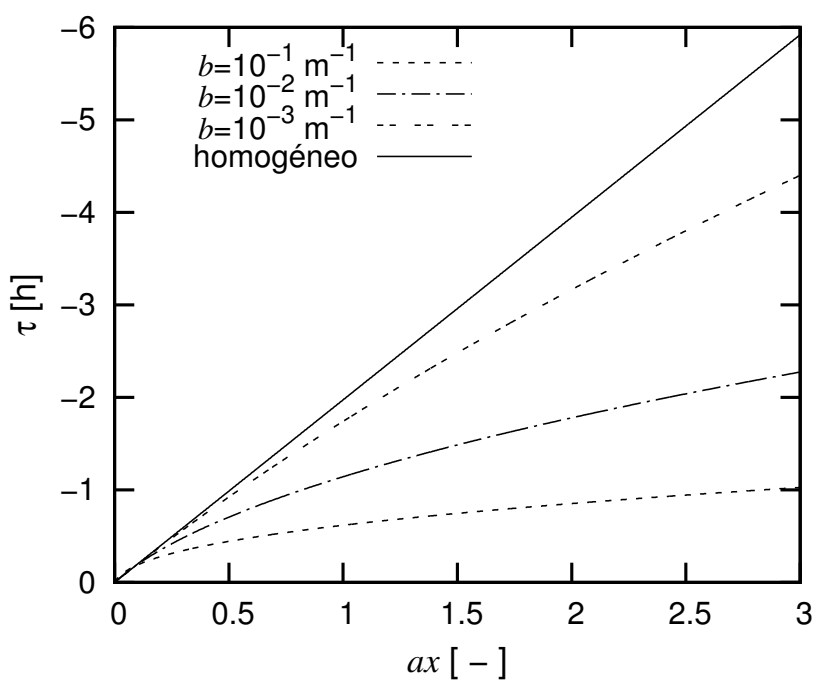

Figura 6.6: Retraso temporal $\tau$ versus distancia adimensional ax para diferentes tasas de incremento de la conductividad hidráulica.

El retraso temporal entre la marea oceánica y la fluctuación inducida en el acuífero heterogéneo puede obtenerse de la ecuación (6.47). La Fig. 6.6 muestra los retrasos temporales como función de la distancia ax para $b=10^{-1}, 10^{-2}, 10^{-3} \mathrm{~m}^{-1}$. Los retrasos para los acuíferos heterogéneos son más pequeños que el correpondiente al acuífero homogéneo que muestra un incremento lineal con la distancia (ecuación (6.26)). El retraso del acuífero heterogéneo se comporta como una función de tipo raíz cuadrada, dando como resultado una mayor velocidad de transmisión de la fluctuación de marea. Como era de esperar, la velocidad de propagación de la marea inducida aumenta con $b$.

Con el objeto de analizar el efecto de los valores extremadamente altos de $K$ predichos por la ecuación (6.27) a medida que $x$ tiende a infinito, se derivará una solución analítica particular para el caso de un acuífero finito de extensión $L$, como se ilustra en la Fig. 6.7. En este caso, el valor máximo de $K$ será finito y estará dado por $K_{0}(1+a L)$. La ecuación (6.28) se resuelve en el dominio finito $0 \leq x \leq L$ con la condición de marea dada por la ecuación (6.29) y la siguiente condición de flujo nulo en el borde derecho del acuífero:

$$
K(x) \frac{\partial h}{\partial x}=0, \quad \text { en } x=L .
$$

Basándonos en un razonamiento similar al seguido en la deducción de la ecuación (6.45), se puede demostrar que la solución analítica del problema de valores de contorno 


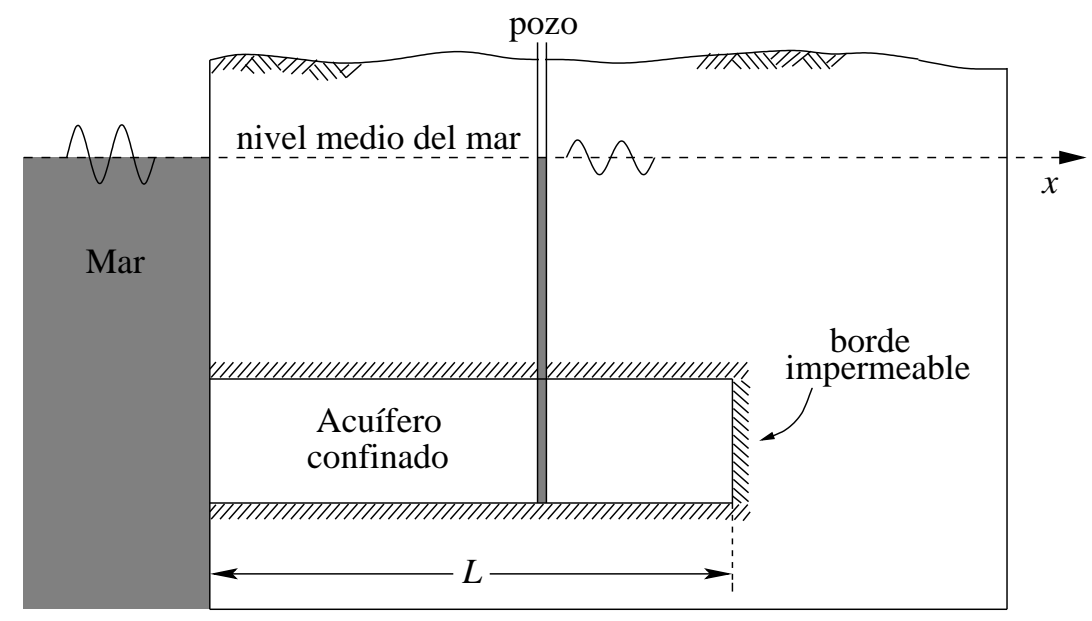

Figura 6.7: Representación esquemática de un acuífero costero finito de longitud $L$.

definido por las ecuaciones (6.28),(6.29) y (6.49) viene dada por:

$$
h(x, t)=\operatorname{Re}\left[A\left\{C_{1} J_{0}(2 \Lambda \sqrt{1+b x})+C_{2} Y_{0}(2 \Lambda \sqrt{1+b x})\right\} e^{i \omega t}\right]
$$

donde

$$
\begin{aligned}
& C_{1}=\frac{Y_{0}^{\prime}(2 \Lambda \sqrt{1+b L})}{J_{0}(2 \Lambda) Y_{0}^{\prime}(2 \Lambda \sqrt{1+b L})-Y_{0}(2 \Lambda) J_{0}^{\prime}(2 \Lambda \sqrt{1+b L})}, \\
& C_{2}=\frac{-J_{0}^{\prime}(2 \Lambda \sqrt{1+b L})}{J_{0}(2 \Lambda) Y_{0}^{\prime}(2 \Lambda \sqrt{1+b L})-Y_{0}(2 \Lambda) J_{0}^{\prime}(2 \Lambda \sqrt{1+b L})} .
\end{aligned}
$$

siendo $J_{0}$ y $Y_{0}$ las funciones de Bessel de orden cero de primera y segunda especie, respectivamente, y siendo $J_{0}^{\prime}$ y $Y_{0}^{\prime}$ sus respectivas derivadas. El coeficiente de amplitud $c$ y el retraso de fase $\tau$ se obtienen a partir de (6.46) y (6.47) utilizando:

$$
\begin{aligned}
& B_{r}=\operatorname{Re}\left[C_{1} J_{0}(2 \Lambda \sqrt{1+b x})+C_{2} Y_{0}(2 \Lambda \sqrt{1+b x})\right] \\
& B_{i}=\operatorname{Im}\left[C_{1} J_{0}(2 \Lambda \sqrt{1+b x})+C_{2} Y_{0}(2 \Lambda \sqrt{1+b x})\right] .
\end{aligned}
$$

La Fig. 6.8 muestra la variación del coeficiente de amplitud con la distancia a la línea de costa, tanto para un acuífero infinito como para acuíferos finitos de extensión adimensional $a L=10$ y $a L=30$. En todos los casos, los parámetros hidráulicos del acuífero son los mismos que los utilizados en el ejemplo de la Fig. 6.4. Como puede observarse, a medida que la extensión adimensional $a L$ aumenta, las amplitudes predichas por la ecuación (6.50) tienden rápidamente al valor de amplitud para un acuífero infinito, dada por la ecuación (6.46). Este test demuestra que las fluctuaciones de altura piezométrica 


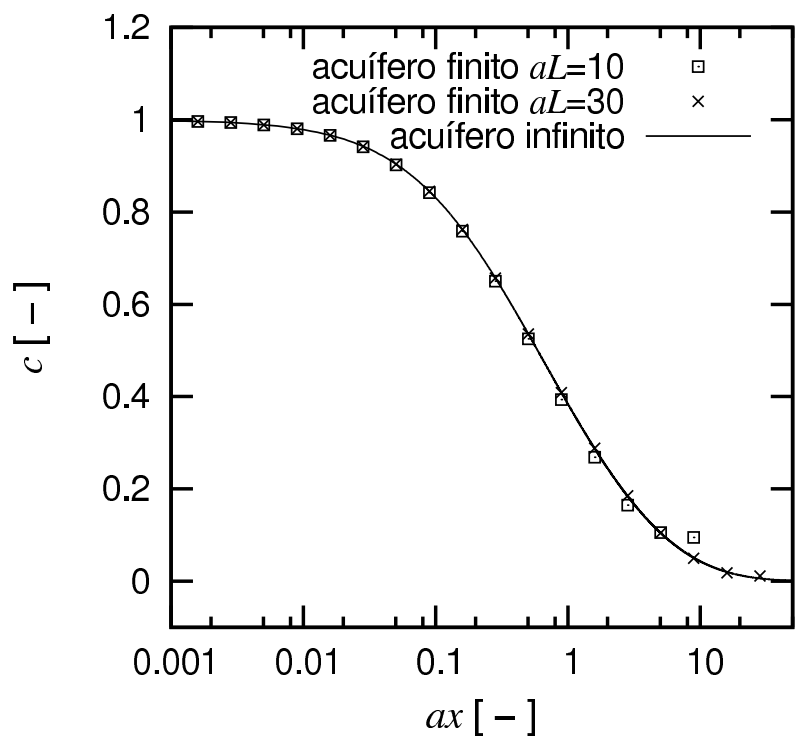

Figura 6.8: Coeficiente de amplitud $c$ versus distancia adimensional ax en escala logarítmica para dos acuíferos finitos y uno infinito.

inducidas por marea no son sensibles a los valores de conductividad hidráulica lejos de la costa, y la ecuación (6.45) es válida aún cuando los valores de $K$ dados por la ecuación (6.27) tienden a infinito.

\subsection{Aproximación asintótica de la solución exacta}

En esta sección se obtendrá una expresión aproximada de la solución exacta (6.45) válida para valores relativamente pequeños de la tasa de aumento de la conductividad hidráulica b. La solución analítica aproximada es válida para:

$$
b<<2^{7 / 2} a \approx 10 a .
$$

Para estos valores de $b$, los argumentos de la función de Hankel de (6.45) satisfacen:

$$
|2 \Lambda \sqrt{1+b x}|>>\frac{1}{4}, \quad|2 \Lambda|>>\frac{1}{4},
$$

por lo que es válido utilizar las siguientes aproximaciones asintóticas [3]:

$$
\begin{gathered}
H_{0}^{(1)}(2 \Lambda \sqrt{1+b x}) \approx[\pi \Lambda \sqrt{1+b x}]^{-1 / 2} e^{i\left[2 \Lambda \sqrt{1+b x}-\frac{1}{4} \pi\right]}, \\
H_{0}^{(1)}(2 \Lambda) \approx[\pi \Lambda]^{-1 / 2} e^{i\left[2 \Lambda-\frac{1}{4} \pi\right]} .
\end{gathered}
$$


Remplazando las ecuaciones (6.56) y (6.57) en (6.45), se obtiene la siguiente solución aproximada:

$$
h(x, t)=A \frac{e^{-2 \frac{a}{b}(\sqrt{1+b x}-1)}}{(1+b x)^{\frac{1}{4}}} \cos \left[-2 \frac{a}{b}(\sqrt{1+b x}-1)+\omega t\right] .
$$

Aunque la validez de la ecuación (6.58) está limitada a valores de $b$ que satisfagan la ecuación (6.54), su expresión matemática es simple y puede ser utilizada para analizar cualitativamente las fluctuaciones de altura piezométrica inducidas por marea. Nótese que, al igual que en la solución de Jacob, las expresiones del coeficiente de amplitud y el retraso temporal están explícitos en la expresión analítica de $h(x, t)$ :

$$
\begin{gathered}
c(x)=\frac{e^{-2 \frac{a}{b}(\sqrt{1+b x}-1)}}{(1+b x)^{\frac{1}{4}}}, \\
\tau(x)=-\frac{1}{\omega}\left[2 \frac{a}{b}(\sqrt{1+b x}-1)\right] .
\end{gathered}
$$

En la ecuación (6.60) puede observarse que la dependencia de $\tau$ con $x$ está dada por una función raíz cuadrada. Esta dependencia verifica el mismo comportamiento observado cualitativamente en el análisis del retraso temporal de la solución analítica exacta (ver Fig. 6.6).

Cuando la heterogeneidad de la conductividad hidráulica es despreciable, esto es $b \rightarrow 0$, se tiene:

$$
\lim _{b \rightarrow 0} \frac{1}{b}(\sqrt{1+b x}-1)=\frac{1}{2} x, \quad \lim _{b \rightarrow 0}(1+b x)^{\frac{1}{4}}=1,
$$

con lo cual las expresiones (6.58), (6.59) y (6.60) convergen a las expresiones (6.24), (6.25) y (6.26) correspondientes a un acuífero homogéneo.

La Fig. 6.9 muestra el coeficiente de amplitud $c$ (izquierda) y el retraso de fase $\tau \omega$ (derecha) de la solución exacta y la aproximada ((6.45) y (6.58)) para dos magnitudes diferentes de la tasa de incremento de la conductividad hidráulica $b$.

Como era de esperar, para un valor de $b$ relativamente pequeño $\left(b=10^{-3} \mathrm{~m}^{-1}\right)$, los valores de amplitud y de retraso de fase $\tau \omega$ predichos por la ecuación (6.58) están en excelente acuerdo con los correspondientes valores predichos por la solución exacta (6.45). Por el contrario, para $b=10^{-1} \mathrm{~m}^{-1}$, la condición dada por la ecuación (6.54) no se satisface y se observan discrepancias significativas entre los valores predichos por ambas soluciones. 

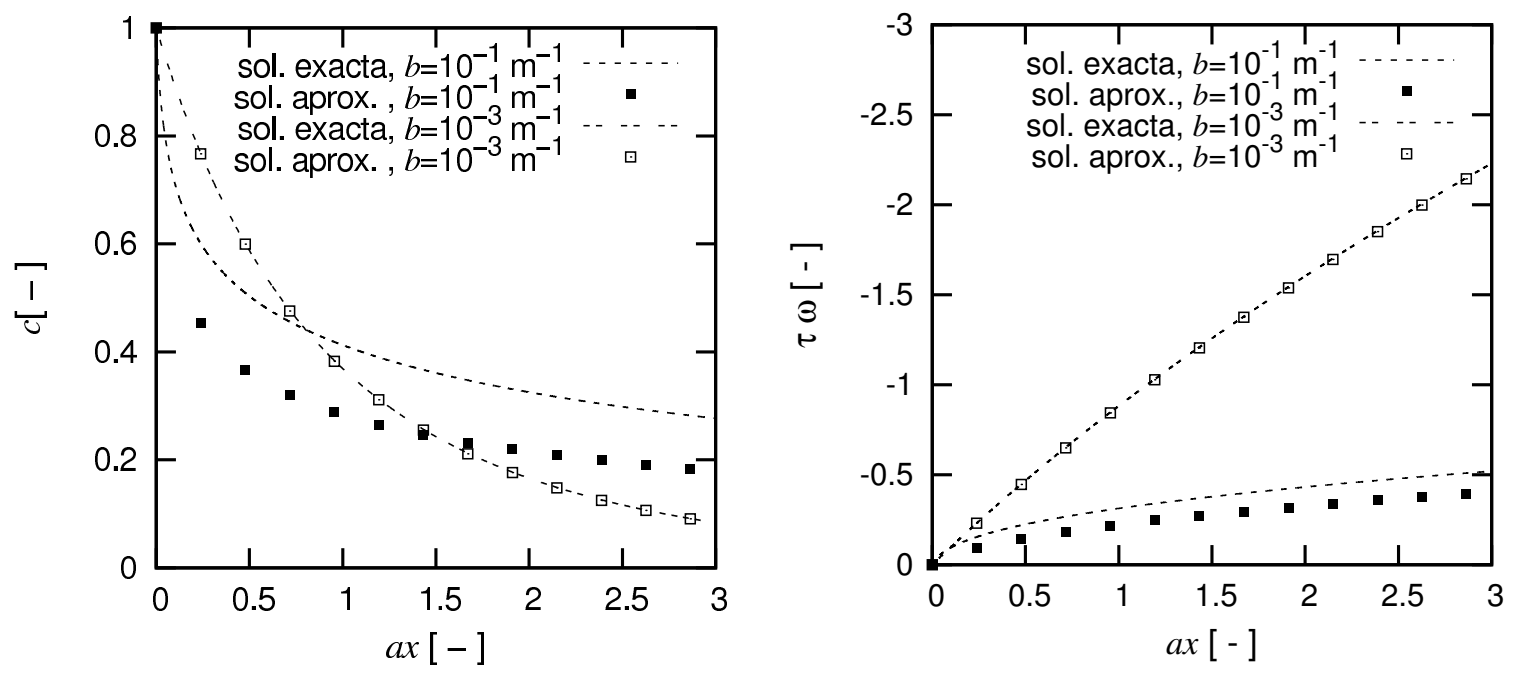

Figura 6.9: Coeficiente de amplitud $c$ (izquierda) y fase $\tau \omega$ (derecha) versus distancia adimensional ax para las soluciones exacta y aproximada.

En la siguiente sección, la solución analítica exacta que hemos presentado será utilizada para estimar la conductividad hidráulica en un acuífero costero donde se dispone de datos reales.

\subsection{Estimación de la conductividad hidráulica mediante el método de marea inducida}

El método de marea inducida tiene por objeto la determinación de los parámetros hidráulicos a partir del análisis de la respuesta del acuífero a las fluctuaciones periódicas inducidas por la marea. Esta respuesta se traduce en cambios espacio-temporales de amplitud y fase en las fluctuaciones de altura piezométrica que pueden ser registrados en pozos cercanos a la línea de costa. Los parámetros hidráulicos se estiman mediante el ajuste de la respuesta de un modelo teórico a los datos de amplitud y fase. El modelo más utilizado en aplicaciones prácticas es el de Jacob [44] debido a su simplicidad (ecuación (6.24)). Como hemos visto, el coeficiente de amplitud $c(x)$ y el retraso temporal $\tau(x)$ correspondientes a este modelo son expresiones analíticas simples, función de la distancia a la línea de costa y del coeficiente de propagación de marea $a$ (ver ecuaciones (6.25) y (6.26)). El ajuste de las expresiones de $c(x)$ y $\tau(x)$ a los datos observados provee dos metodologías de cálculo diferentes denominadas de amplitud y de fase, respectivamente. 
Estas metodologías permiten estimar el cociente $D=K / S_{s}$, denominado comúnmente $d i$ fusividad hidráulica [7]. Para obtener el valor de la conductividad hidráulica es necesario contar además con un valor del almacenamiento específico del acuífero $\mathrm{S}_{s}$.

Las ecuaciones (6.46) y (6.47) que se han presentado en la sección 6.4 pueden utilizarse como un modelo alternativo al de Jacob en el caso de existir variaciones monótonas en la conductividad hidráulica, como en el caso de acuíferos aluviales. El ajuste de estas expresiones permitirá validar o refutar la hipótesis del aumento de la conductividad hidráulica con la distancia a la línea de costa. En la sección siguiente se presentan los resultados obtenidos en un caso real a partir del ajuste de los parámetros $K_{0}$ y $b$.

\subsubsection{Resultados}

Los datos utilizados para realizar el ajuste fueron publicados por Schultz y Ruppel [93]. Los mismos corresponden a mediciones de altura piezométrica inducida por marea (de componente mayoritariamente semidiurna) en un estuario de la Isla de Sapelo, Georgia, EEUU. Las mediciones se realizaron en 8 pozos alineados en forma perpendicular a la línea de costa, cubriendo una distancia total de unos $60 \mathrm{~m}$. El almacenamiento específico $\mathrm{S}_{s}$ del acuífero fue estimado en $\mathrm{S}_{s}=0.016 \mathrm{~m}^{-1}$ [93]. En el mencionado trabajo se realizan estimaciones de conductividad hidráulica mediante el método de marea utilizando el modelo de Jacob [44]. Para cada uno de los 8 pozos los autores estiman la conductividad hidráulica a partir de los valores del coeficiente de amplitud y el desfasaje temporal entre la señal de marea registrada sobre la línea de costa y la registrada en el pozo. Los resultados que los autores obtienen mediante la utilización de los métodos de amplitud y de fase discrepan ampliamente. Esto se debe principalmente a la alta heterogeneidad de la condutividad hidráulica presente en la zona.

En la Fig. 6.10 se muestra el resultado del ajuste del modelo propuesto de conductividad lineal y el modelo homogéneo de Jacob a los datos de amplitud (a) y fase (b), expresados en términos del coeficiente de amplitud $c$ y del retraso temporal $\tau$, respectivamente. Para ambos modelos el ajuste se realizó mediante un método de búsqueda exhaustiva, explorando todo el espacio de parámetros posibles. En la Tabla 6.1 se listan los valores obtenidos para los parámetros del modelo lineal $\left(K_{0}\right.$ y b) y el homogéneo $(K)$.

Como puede observarse en la Fig. 6.10, el modelo de conductividad lineal predice satisfactoriamente los valores de $c$ y $\tau$ observados en todo el rango de distancias a la línea 
(a)

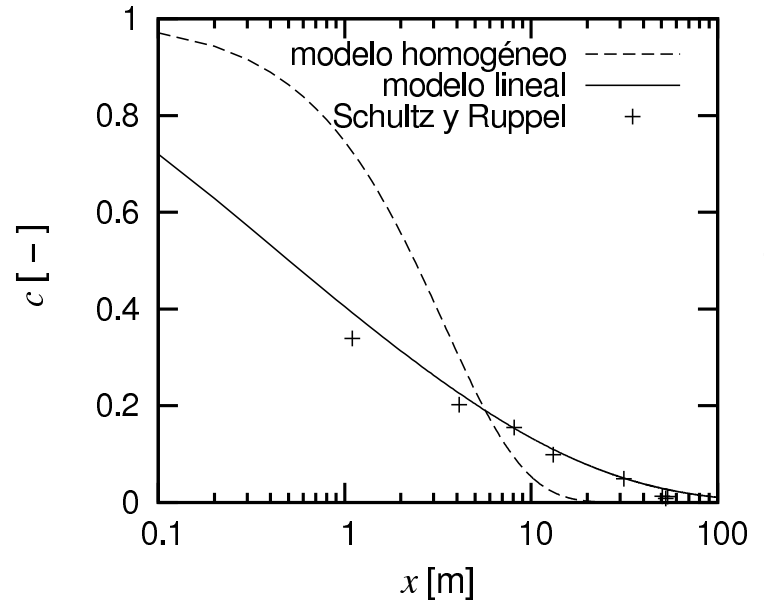

(b)

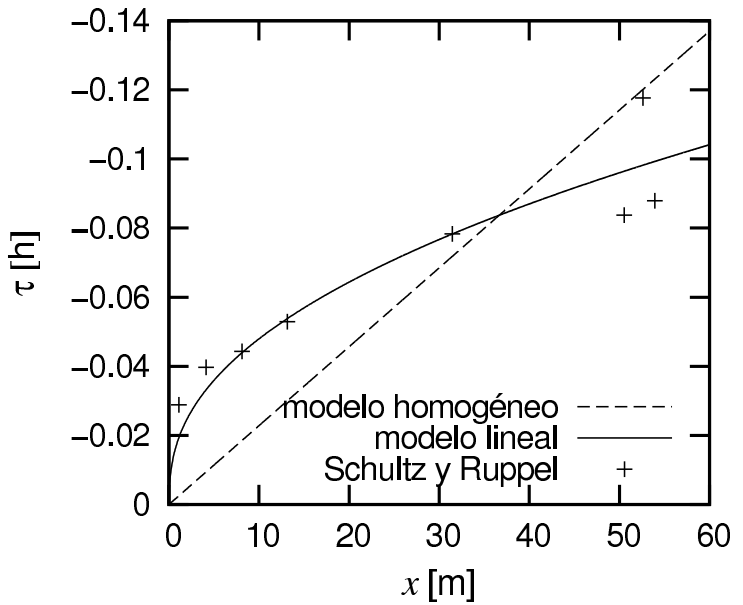

Figura 6.10: Ajustes obtenidos para (a) $c(x)$ y (b) $\tau(x)$. La línea continua corresponde al modelo lineal, la línea de trazos al modelo homogéneo y los puntos a los datos de Schultz y Ruppel [93].

\begin{tabular}{|l|c|c|c|}
\hline \multirow{2}{*}{} & \multicolumn{2}{|c|}{ Modelo lineal } & Modelo homogéneo \\
\cline { 2 - 4 } & $K_{0}[\mathrm{~m} / \mathrm{s}]$ & $b\left[\mathrm{~m}^{-1}\right]$ & $K[\mathrm{~m} / \mathrm{s}]$ \\
\hline Amplitud & $1.1 \times 10^{-6}$ & 49.82 & $1.37 \times 10^{-5}$ \\
\hline Fase & $1.93 \times 10^{-6}$ & 119.7 & $1.58 \times 10^{-3}$ \\
\hline
\end{tabular}

Tabla 6.1: Parámetros ajustados mediante los métodos de amplitud y de fase.

de costa, siendo la discrepancia entre las estimaciones obtenidas por ambos métodos relativamente pequeña, en particular para $K_{0}$ (ver Tabla 6.1). Por su parte, las estimaciones obtenidas mediante el ajuste del modelo homogéneo discrepan en 2 órdenes de magnitud (ver Tabla 6.1) y como puede observarse en la Fig. 6.10 el ajuste dista mucho de ser satisfactorio. Estos resultados muestran la validez del modelo lineal para representar las fluctuaciones observadas, tanto en el dominio de la amplitud como en el de la fase. A pesar de tratarse de un modelo sencillo de variación de $K$ con la distancia, los resultados obtenidos contribuyen a mejorar conceptualmente el modelo hidrológico regional. En este sentido resulta importante destacar que la determinación de los parámetros hidráulicos a escala de campo es tan o más difícil que la determinación que se realiza a escala de 
laboratorio.

Por útlimo, cabe señalar que los valores de $b / a$ calculados a partir de los ajustes obtenidos con ambos métodos no satisfacen la relación (6.54) $(b / a=49.32$ para el método de amplitud y $b / a=157.5$ para el método de fase). Por esta razón, la solución analítica aproximada no es aplicable en este caso particular.

\subsection{Conclusiones}

En este Capítulo se han analizado las fluctuaciones de altura piezométrica inducidas por marea en un acuífero costero confinado cuya conductividad hidráulica aumenta linealmente con la distancia a la costa. A partir de un modelo matemático que describe la interacción hidráulica entre el mar y el acuífero, se derivó una solución analítica exacta que predice las fluctuaciones de altura hidráulica en términos de una función de Hankel. Para valores pequeños de la tasa de aumento de conductividad hidráulica se obtuvo una solución analítica aproximada, cuya expresión matemática es simple. En términos generales, se puede concluir que la existencia de una variación lineal de $K$ produce los siguientes efectos: (1) disminución de amplitud para distancias menores que la distancia característica $a^{-1},(2)$ aumento de la amplitud para grandes distancias $\left(x>a^{-1}\right)$ y (3) transmición más rápida del efecto de marea, determinada por una curva de retraso temporal que puede aproximarse con una función de tipo raíz cuadrada. Mediante ejemplos teóricos hipotéticos se mostró que la influencia de las variaciones de conductividad hidráulica puede ser significativa y debe ser tenida en cuenta en el estudio de aquellos acuíferos costeros en los que la conductividad hidráulica aumenta con la distancia a la línea de costa. Finalmente, la solución analítica exacta fue utilizada para estimar la conductividad hidráulica en un caso real. A diferencia de las estimaciones realizadas mediante la utilización de la fórmula clásica de Jacob, los valores de conductividad hidráulica obtenidos utilizando el modelo lineal son consistentes tanto con los datos de amplitud como con los de fase, mostrando la validez del modelo teórico propuesto. 


\section{Capítulo 7}

\section{Conclusiones}

En este Capítulo se resumen los resultados más relevantes del trabajo de Tesis, resaltando aquellos que constituyen aportes originales a los temas abordados.

Con el fin de caracterizar el flujo en medios porosos heterogéneos, se implementó una técnica numérica para el cálculo de las relaciones constitutivas en medios porosos bidimensionales y tridimensionales. Esta técnica se basa en la simulación del flujo no saturado en estado estacionario bajo condiciones similares a las que suelen someterse las muestras de roca en experimentos de laboratorio. Para simular el flujo bajo estas condiciones se estableció un problema con condiciones de contorno basado en la ecuación de Richards, que fue resuelto utilizando un método mixto de elementos finitos. Este método posee la ventaja de aproximar con igual precisión los campos de altura de presión y de flujo, garantizando además la conservación local de la masa.

La técnica numérica propuesta fue utilizada para caracterizar el flujo no saturado en muestras sintéticas de roca fracturada y calcular las correspondientes curvas de conductividad hidráulica relativa y de saturación efectiva. La obtención de estas curvas posee importancia ya que su medición presenta numerosas limitaciones prácticas y los datos experimentales son prácticamente inexistentes.

La técnica propuesta fue aplicada al cálculo de la permeabilidad efectiva en rocas bidimensionales con heterogeneidades de tipo fractal. Esta aplicación particular demostró la utilidad e importancia de la técnica en el estudio de las propiedades sísmicas de medios porosos heterogéneos y permitió verificar que en el límite de bajas frecuencias la permeabilidad sísmica coincide con la permeabilidad hidráulica efectiva. Por último se realizó un análisis comparativo entre las conductividades hidráulicas efectivas estimadas mediante experimentos bidimensionales y tridimensionales en rocas con heterogeneidades de tipo lognormal. Este análisis permitió cuantificar el error que se comete al aproximar un medio tridimensional por uno bidimensional.

La caracterización hidráulica de rocas fracturadas también fue abordada mediante modelos analíticos. Utilizando conceptos de geometría fractal para representar la red de 
fracturas, se derivó un modelo constitutivo analítico válido para la caracterización del flujo bifásico inmiscible en rocas fracturadas. El modelo obtenido consiste en expresiones analíticas cerradas para las curvas de saturación efectiva y de permeabilidad relativa de ambas fases. Estas expresiones dependen de cinco parámetros independientes que tienen significado geométrico y físico. Esta característica constituye uno de los rasgos más atractivos del modelo, puesto que permite evaluar las propiedades hidráulicas de la roca en términos de las propiedades de la red de fracturas. Las curva de permeabilidad relativa del agua fue comparada con valores obtenidos mediante simulación numérica, mientras que la curva de permeabilidad del aire fue comparada con datos experimentales de rocas cristalinas medidos en Grimsel Test Site (Suiza). En ambos casos se encontraron muy buenos ajustes para el rango completo de saturación efectiva y sobre varios órdenes de magnitud de las permeabilidades del agua y del aire. Por otra parte, utilizando las curvas de saturación efectiva y de permeabilidad relativa de agua bajo la hipótesis de Richards se obtuvo un modelo constitutivo válido para la descripción del flujo no saturado de agua. Este modelo fue validado mediante su comparación con el modelo clásico de Burdine, encontrándose que bajo ciertas hipótesis ambos modelos son equivalentes. Por último el modelo fue contrastado con las relaciones constitutivas simuladas en rocas fracturadas bidimensionales y tridimensionales, lográndose una muy buena representación de los valores simulados.

A escala de campo se estudiaron los efectos producidos por la presencia de heterogeneidades de la conductividad hidráulica sobre el flujo inducido por marea en acuíferos costeros confinados. Asumiendo que la conductividad hidráulica aumenta linealmente con la distancia a la costa, se derivó una solución analítica exacta que permite predecir las fluctuaciones de altura piezométrica observables en pozos costeros. Esta solución se expresa en términos de una función de Hankel. Para valores pequeños de la tasa de aumento de la conductividad hidráulica se obtuvo además una solución analítica aproximada. Esta solución posee una expresión matemática simple, lo cual constituye una ventaja respecto a la solución exacta, puesto que permite interpretar de manera sencilla el efecto de la heterogeneidad sobre la amplitud y la fase de las fluctuaciones de altura piezométrica. El análisis del efecto de la heterogeneidad lineal sobre las fluctuaciones de altura piezométrica fue realizado mediante ejemplos hipotéticos, comparando las curvas del coeficiente de amplitud y del retraso de fase con las correspondientes al caso homogéneo. El análisis permitió concluir que la presencia de la heterogeneidad produce 
una disminución de amplitud para distancias menores a una distancia característica, y un aumento de amplitud para distancias mayores. Para todo el rango de distancias se observa además una disminución del retraso de fase, cuya dependencia con la distancia puede aproximarse con una función de tipo raíz cuadrada, resultando en una mayor velocidad de transmisión del efecto de marea. Los efectos observados en la amplitud y la fase se acentúan con el aumento de la tasa de incremento de la conductividad hidráulica, lo cual permite concluir que la influencia de la heterogeneidad puede ser significativa y debe ser tenida en cuenta en el estudio de aquellos acuíferos de origen aluvial donde este tipo de heterogeneidad suele estar presente. Finalmente, la solución analítica exacta fue utilizada para estimar la conductividad hidráulica en un caso real mediante el método de marea inducida. A diferencia de las estimaciones realizadas mediante la utilización de la fórmula clásica de Jacob, los valores de conductividad hidráulica obtenidos utilizando el modelo lineal son consistentes tanto con los datos de amplitud como con los de fase, mostrando la validez del modelo teórico propuesto.

En síntesis, en el presente trabajo de Tesis se han desarrollado herramientas numéricas y se han derivado nuevas soluciones analíticas que permiten una mejor descripción del flujo en medios porosos heterogéneos a escala de laboratorio y de campo. Los resultados han sido publicados en revistas de la especialidad y conforman una base teórica sólida para la realización de distintos trabajos a futuro. 
Capítulo 7. Conclusiones 


\section{Agradecimientos}

En primer lugar quiero expresar mi más sincero agradecimiento a mi director, el Dr.Luis Guarracino, por su apoyo incondicional y su generosidad. Gracias por brindarme en forma desinteresada todo tu conocimiento y principalmente gracias por la confianza que has depositado en mi y por todo el tiempo que has invertido para que esta Tesis se materialice.

A los miembros del jurado, la Dra. Ana María Platzeck, el Dr. J. Germán Rubino y el Dr. Luis Vives. Gracias por sus valiosísimas correcciones y sugerencias que sin duda enriquecen el contenido de esta Tesis.

Al CONICET y a la Facultad de Ciencias Astronómicas y Geofísicas por la financiación completa de mi carrera de posgrado.

A todos los amigos del Departamento de Geofísica Aplicada, en especial a Andrés,

Juani y Claudia, por la confianza que han depositado en mi y porque nunca me negaron su ayuda, GRACIAS!!!.

A todos mis amigos de la vida, en especial a Tomás, Emilio, Marcelo, Seba, Fede, Pablo y Nico. Gracias por su amistad y por quererme como soy.

Por último quiero agradecer a mi familia: Diana, Bruno, Silvi y Guille. Ellos han sido mi verdadero y único motor para llevar a cabo, a pesar de todo, esta Tesis doctoral. A ellos es a quienes dedico esta Tesis, y a quienes dedicaré todo mi trabajo, hoy y siempre. 


\section{Apéndice A}

\section{Resolución numérica del experimento 3D}

En este Apéndice se establece el problema diferencial para modelar el flujo estacionario de agua en un medio poroso parcialmente saturado en las condiciones del experimento descrito en la sección (2.5.2). Se presenta el método utilizado para la discretización espacial y la implementación del algoritmo en un dominio tridimensional. La discretización espacial se realiza empleando un método mixto de elementos finitos que permite aproximar en forma simultánea tanto la altura de presión $h$ como el flujo de agua q.

\section{A.1 Planteo matemático del experimento}

Para describir matemáticamente el problema consideremos una muestra tridimensional con forma de prisma rectangular que ocupa el dominio $\Omega=\left[0, L_{x}\right] \times\left[0, L_{y}\right] \times\left[0, L_{z}\right]$ con frontera $\Gamma=\Gamma^{L} \cup \Gamma^{R} \cup \Gamma^{F} \cup \Gamma^{B} \cup \Gamma^{D} \cup \Gamma^{U}$ como se ilustra en la Fig. A.1. Los

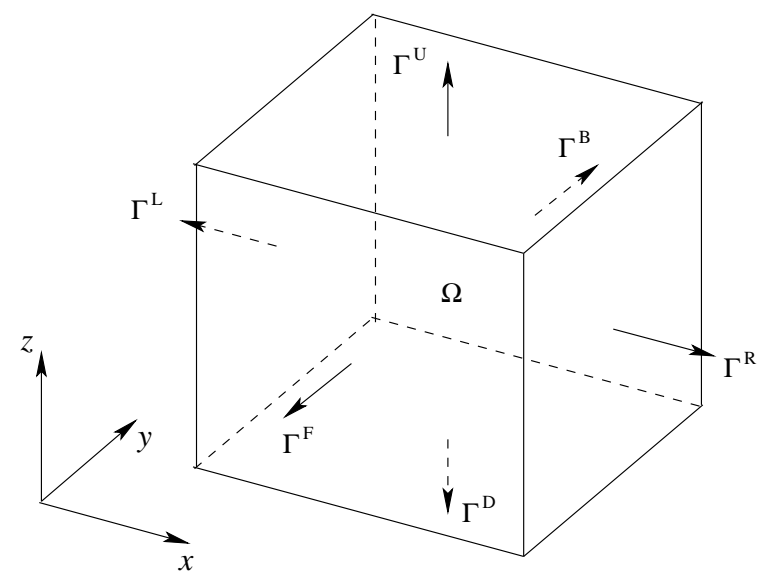

Figura A.1: Dominio de simulación.

supraíndices indican el nombre de la cara según su ubicación respecto a los ejes coordenados ${ }^{1}$. El problema diferencial que gobierna el flujo no saturado bajo las condiciones

\footnotetext{
${ }^{1}$ del inglés Left (izquierda), Right (derecha), Front (adelante), Back (atrás), Down (abajo), Up (arriba).
} 
del experimento descrito en la sección (2.5.2) puede plantearse del siguiente modo: Hallar $(h, \mathbf{q}): \Omega \times \Omega \rightarrow \mathbb{R} \times \mathbb{R}^{3}$ tal que:

$$
\begin{aligned}
& \boldsymbol{\nabla} \cdot \mathbf{q}=0, \quad(x, y, z) \in \Omega, \\
& \mathbf{q}=-K(h) \boldsymbol{\nabla}(h+z), \quad(x, y, z) \in \Omega, \\
& h=h^{D}, \quad(x, y, z) \in \Gamma^{D}, \\
& h=h^{U}, \quad(x, y, z) \in \Gamma^{U}, \\
& \mathbf{q} \cdot \boldsymbol{\nu}=0, \quad(x, y, z) \in \Gamma^{L} \cup \Gamma^{R} \cup \Gamma^{F} \cup \Gamma^{B},
\end{aligned}
$$

donde $\boldsymbol{\nu}$ es el versor normal externo a la cara considerada. Dado que $h$ interviene en forma no lineal, se procede a linealizar el problema mediante un método iterativo de Picard [21]. El esquema iterativo se define del siguiente modo:

$$
\begin{aligned}
& \boldsymbol{\nabla} \cdot \mathbf{q}^{i+1}=0, \quad(x, y, z) \in \Omega, \\
& \mathbf{q}^{i+1}=-K\left(h^{i}\right) \boldsymbol{\nabla}\left(h^{i+1}+z\right), \quad(x, y, z) \in \Omega, \\
& h^{i+1}=h^{D}, \quad(x, y, z) \in \Gamma^{D}, \\
& h^{i+1}=h^{U}, \quad(x, y, z) \in \Gamma^{U}, \\
& \mathbf{q}^{i+1} \cdot \boldsymbol{\nu}=0, \quad(x, y, z) \in \Gamma^{L} \cup \Gamma^{R} \cup \Gamma^{F} \cup \Gamma^{B} .
\end{aligned}
$$

donde el supraíndice $i$ indica el nivel de iteración. Notar que para implementar el método es preciso establecer un valor inicial de iteración $h^{0}$. Luego el problema (A.6)-(A.10) se resuelve en forma iterativa hasta alcanzar la convergencia. En la sección siguiente se planteará la formulación mixta débil del problema (A.6)-(A.10).

\section{A.2 Formulación mixta débil}

Los espacios de funciones que se utilizarán en la formulación mixta débil son los siguientes:

$$
\begin{gathered}
V=H(\operatorname{div}, \Omega)=\left\{\mathbf{v} \in\left[L^{2}(\Omega)\right]^{3}: \nabla . \mathbf{v} \in L^{2}(\Omega)\right\} \\
V_{0}=\left\{\mathbf{v} \in V: \mathbf{v} . \boldsymbol{\nu}=0, \quad(x, y, z) \in \Gamma^{L} \cup \Gamma^{R} \cup \Gamma^{F} \cup \Gamma^{B}\right\} \\
W=L^{2}(\Omega)
\end{gathered}
$$

donde el espacio de funciones vectoriales $V$ será utilizado para aproximar q y el espacio de funciones escalares $W$ para aproximar $h$. 
Para obtener la forma débil se multiplican las ecuaciones (A.6) y (A.7) por las funciones de prueba $\psi \in W$ y $\mathbf{v} \in V_{0}$ respectivamente, para luego integrar las expresiones resultantes sobre el dominio completo $\Omega$. De esta manera, la formulación mixta débil del problema (A.6)-(A.10) se plantea del siguiente modo: Hallar $\left(h^{i+1}, \mathbf{q}^{i+1}\right) \in W \times V$ tal que:

$$
\begin{aligned}
& \left(\boldsymbol{\nabla} \cdot \mathbf{q}^{i+1}, \psi\right)_{\Omega}=0, \quad \psi \in W, \\
& \left(\frac{\mathbf{q}^{i+1}}{K\left(h^{i}\right)}, \mathbf{v}\right)_{\Omega}-\left(h^{i+1}+z, \boldsymbol{\nabla} \cdot \mathbf{v}\right)_{\Omega} \\
& +\left\langle h^{D}, \mathbf{v} \cdot \boldsymbol{\nu}\right\rangle_{\Gamma^{D}}+\left\langle h^{U}+L_{z}, \mathbf{v} \cdot \boldsymbol{\nu}\right\rangle_{\Gamma^{U}}=0, \quad \mathbf{v} \in V_{0} .
\end{aligned}
$$

El siguiente paso para resolver el sistema (A.14) -(A.15) será definir un esquema de elementos finitos mixtos.

\section{A.3 Método mixto de elementos finitos}

Consideremos una partición regular no solapada de $\Omega$ en subdominios con forma de prisma rectangular $\Omega_{j, k, l}$ tal que:

$$
\Omega=\bigcup_{j=1, k=1, l=1}^{N_{x}, N_{y}, N_{z}} \Omega_{j, k, l} \quad \text { con } \quad \Omega_{j, k, l}=\left[x_{j}, x_{j+1}\right] \times\left[y_{k}, y_{k+1}\right] \times\left[z_{l}, z_{l+1}\right],
$$

donde $N_{x}, N_{y}$ y $N_{z}$ representan el número de subdominios en las direcciones $x, y$ y $z$, respectivamente.

En la Fig. A.2 se muestra un elemento genérico $\Omega_{j, k, l}$ de la partición, cuyo borde es $\Gamma_{j, k, l}=\Gamma_{j, k, l}^{1} \cup \Gamma_{j+1, k, l}^{1} \cup \Gamma_{j, k, l}^{2} \cup \Gamma_{j, k+1, l}^{2} \cup \Gamma_{j, k, l}^{3} \cup \Gamma_{j, k, l+1}^{3}$. Notar que se han utilizado los supraíndices 1,2 y 3 para diferenciar entre las caras normales a los tres ejes coordenados $x, y$ y $z$, respectivamente.

Para realizar la formulación híbrida del problema (ver Capítulo 3) se definen los siguientes espacios de elementos finitos de Raviart-Thomas-Nedelec de índice $m$ [83, 76]:

$$
\begin{gathered}
\Lambda^{m}=\left\{\lambda^{s}:\left.\lambda^{s}\right|_{\Gamma_{j, k, l}^{s}}=\lambda_{j, k, l}^{s} \in P_{m}\left(\Gamma_{j, k, l}^{s}\right), s=1,2,3\right\}, \\
V_{-1}^{m}=\left\{\mathbf{v} \in\left[L^{2}(\Omega)\right]^{3}:\left.\mathbf{v}\right|_{\Omega_{j, k, l}} \in R^{m}\right\}, \\
V_{0,-1}^{m}=\left\{\mathbf{v} \in V_{-1}^{m}: \mathbf{v} \cdot \boldsymbol{\nu}=0 \quad(x, y, z) \in \Gamma^{D} \cup \Gamma^{U}\right\}, \\
W^{m}=\left\{\psi \in L^{2}(\Omega):\left.\psi\right|_{\Omega_{j, k, l}} \in P_{m}\right\},
\end{gathered}
$$




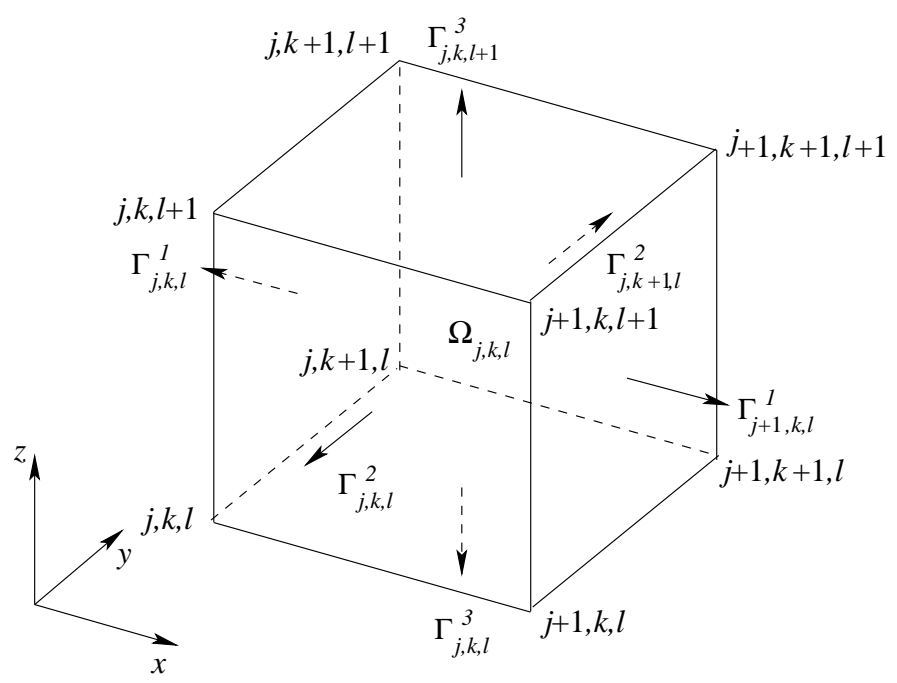

Figura A.2: Elemento $\Omega_{j, k, l}$ de la partición de elementos finitos.

donde $R^{m}=P_{m+1, m, m} \times P_{m, m+1, n} \times P_{m, m, n+1}$ siendo $P_{l, m, n}$ polinomios definidos sobre $\Omega_{j, k, l}$ de grado no mayor que $l, m$ y $n$ en las variables $x, y$ y $z$, respectivamente. El espacio $\Lambda^{m}$ representa un espacio de multiplicadores de Lagrange cuyos elementos $\lambda_{j, k, l}^{s}$ $(s=1,2,3)$ están asociados al valor de altura hidráulica $\left(\lambda_{j, k, l}^{s} \sim h+z\right)$ en los bordes interiores $\Gamma_{j, k, l}^{s}$.

El procedimiento mixto híbrido de elementos finitos se establece del siguiente modo: Dados $\left(h^{i}, \mathbf{q}^{i}, \lambda^{i}\right) \in W^{m} \times V_{0,-1}^{m} \times \Lambda^{m}$, hallar $\left(h^{i+1}, \mathbf{q}^{i+1}, \lambda^{i+1}\right) \in W^{m} \times V_{0,-1}^{m} \times \Lambda^{m}$ tal que:

$$
\begin{gathered}
\sum_{j=1, k=1, l=1}^{N_{x}, N_{y}, N_{z}}\left(\boldsymbol{\nabla} \cdot \mathbf{q}^{i+1}, \psi\right)_{\Omega_{j, k, l}}=0, \quad \psi \in W^{m}, \\
\sum_{j=1, k=1, l=1}^{N_{x}, N_{y}, N_{z}}\left(\frac{\mathbf{q}^{i+1}}{K\left(h^{i}\right)}, \mathbf{v}\right)_{\Omega_{j, k}}-\sum_{j=1, k=1, l=1}^{N_{x}, N_{y}, N_{z}}\left(h^{i+1}+z, \boldsymbol{\nabla} \cdot \mathbf{v}\right)_{\Omega_{j, k, l}} \\
+\left\langle h^{D}, \mathbf{v} \cdot \boldsymbol{\nu}\right\rangle_{\Gamma^{D}}+\left\langle h^{U}+L_{z}, \mathbf{v} \cdot \boldsymbol{\nu}\right\rangle_{\Gamma^{U}} \\
+\sum_{j=2, k=1, l=1}^{N_{x}, N_{y}, N_{z}}\left\langle\lambda_{j, k, l}^{1, i+1}, \mathbf{v} \cdot \boldsymbol{\nu}\right\rangle_{\Gamma_{j, k, l}^{1}, N_{z}}+\sum_{j=1, k=2, l=1}^{N_{x}, N_{y}, N_{z}}\left\langle\lambda_{j, k, l}^{2, i+1}, \mathbf{v} \cdot \boldsymbol{\nu}\right\rangle_{\Gamma_{j, k, l}^{2}} \\
+\sum_{j=1, k=1, l=2}\left\langle\lambda_{j, k, l}^{3, i+1}, \mathbf{v} \cdot \boldsymbol{\nu}\right\rangle_{\Gamma_{j, k, l}^{3}}=0, \quad \mathbf{v} \in V_{0,-1}^{m},
\end{gathered}
$$


Apéndice A. Resolución numérica del experimento 3D

$$
\begin{aligned}
& \sum_{j=2, k=1, l=1}^{N_{x}, N_{y}, N_{z}}\left\langle\mu, \mathbf{q}^{i+1} \cdot \boldsymbol{\nu}\right\rangle_{\Gamma_{j, k, l}^{1}}+\sum_{j=1, k=2, l=1}^{N_{x}, N_{y}, N_{z}}\left\langle\mu, \mathbf{q}^{i+1} . \boldsymbol{\nu}\right\rangle_{\Gamma_{j, k, l}^{2}} \\
& +\sum_{j=1, k=1, l=2}^{N_{x}, N_{y}, N_{z}}\left\langle\mu, \mathbf{q}^{i+1} \cdot \boldsymbol{\nu}\right\rangle_{\Gamma_{j, k, l}^{3}}=0, \quad \mu \in \Lambda^{m}
\end{aligned}
$$

La continuidad de las componentes normales del flujo a través de los bordes internos se ha impuesto mediante la ecuación (A.23).

\section{A.4 Implementación numérica}

Para la implementación numérica se utilizaron los espacios de Raviart-Thomas-Nedelec de orden cero $\left(\mathrm{RTN}_{0}\right)$ :

$$
\begin{gathered}
V_{-1}^{j, k, l}=\operatorname{span}\left\{\mathbf{v}_{j, k, l}^{L}, \mathbf{v}_{j, k, l}^{R}, \mathbf{v}_{j, k, l}^{F}, \mathbf{v}_{j, k, l}^{B}, \mathbf{v}_{j, k, l}^{D}, \mathbf{v}_{j, k, l}^{U}\right\} \\
W^{j, k, l}=\operatorname{span}\left\{\psi_{j, k, l}\right\}
\end{gathered}
$$

donde

$$
\begin{aligned}
& \mathbf{v}_{j, k, l}^{L}(x, y, z)= \begin{cases}\left(-1+\frac{x-x_{j}}{\Delta x_{j}}, 0,0\right) & (x, y, z) \in \Omega_{j, k, l} \\
0 & (x, y, z) \notin \Omega_{j, k, l}\end{cases} \\
& \mathbf{v}_{j, k, l}^{R}(x, y, z)= \begin{cases}\left(\frac{x-x_{j}}{\Delta x_{j}}, 0,0\right) & (x, y, z) \in \Omega_{j, k, l} \\
0 & (x, y, z) \notin \Omega_{j, k, l}\end{cases} \\
& \mathbf{v}_{j, k, l}^{F}(x, y, z)= \begin{cases}\left(0,-1+\frac{y-y_{k}}{\Delta y_{k}}, 0\right) & (x, y, z) \in \Omega_{j, k, l} \\
0 & (x, y, z) \notin \Omega_{j, k, l}\end{cases} \\
& \mathbf{v}_{j, k, l}^{B}(x, y, z)= \begin{cases}\left(0, \frac{y-y_{k}}{\Delta y_{k}}, 0\right) & (x, y, z) \in \Omega_{j, k, l} \\
0 & (x, y, z) \notin \Omega_{j, k, l}\end{cases} \\
& \mathbf{v}_{j, k, l}^{D}(x, y, z)= \begin{cases}\left(0,0,-1+\frac{z-z_{l}}{\Delta z_{l}}\right) & (x, y, z) \in \Omega_{j, k, l} \\
0 & (x, y, z) \notin \Omega_{j, k, l}\end{cases} \\
& \mathbf{v}_{j, k, l}^{U}(x, y, z)= \begin{cases}\left(0,0, \frac{z-z_{l}}{\Delta z_{l}}\right) & (x, y, z) \in \Omega_{j, k, l} \\
0 & (x, y, z) \notin \Omega_{j, k, l}\end{cases}
\end{aligned}
$$


Apéndice A. Resolución numérica del experimento 3D

siendo $\Delta x_{j}=x_{j+1}-x_{j}, \Delta y_{k}=y_{k+1}-y_{k}$ y $\Delta z_{l}=z_{l+1}-z_{l}$.

La solución de (A.21)-(A.23) en cualquier punto del dominio se expresa como:

$$
\begin{gathered}
h^{i+1}(x, y, z)=\sum_{j=1, k=1, l=1}^{N_{x}, N_{y}, N_{z}} h_{j, k, l}^{i+1} \psi_{j, k, l}(x, y, z), \\
\mathbf{q}^{i+1}(x, y, z)=\sum_{j=1, k=1, l=1}^{N_{x}, N_{y}, N_{z}}\left[q_{j, k, l}^{L, i+1} \mathbf{v}_{j, k, l}^{L}(x, y, z)+q_{j, k, l}^{R, i+1} \mathbf{v}_{j, k, l}^{R}(x, y, z)\right. \\
+q_{j, k, l}^{F, i+1} \mathbf{v}_{j, k, l}^{F}(x, y, z)+q_{j, k, l}^{B, i+1} \mathbf{v}_{j, k, l}^{B}(x, y, z) \\
\left.+q_{j, k, l}^{D, i+1} \mathbf{v}_{j, k, l}^{D}(x, y, z)+q_{j, k, l}^{U, i+1} \mathbf{v}_{j, k, l}^{U}(x, y, z)\right],
\end{gathered}
$$

donde $h_{j, k, l}^{i+1}$ se asocia al punto medio del elemento $\Omega_{j, k, l}$ y los flujos normales $q_{j, k, l}^{S, i+1}(S=$ $L, R, F, B, D, U)$ al punto medio de la cara respectiva señalada por su supraíndice. Como ya se ha mencionado, los valores de $\lambda_{j, k, l}^{s}$ están asociados a los de $h+z$ sobre los bordes internos del elemento.

Seleccionando $\mathbf{v}=\mathbf{v}^{L}, \mathbf{v}^{R}, \mathbf{v}^{F}, \mathbf{v}^{B}, \mathbf{v}^{D}$ y $\mathbf{v}^{U}$ en (A.22) y utilizando una regla trapezoidal para calcular el primer término de esta ecuación, obtenemos las siguientes expresiones para $q_{j, k, l}^{L, i+1}, q_{j, k, l}^{R, i+1}, q_{j, k, l}^{F, i+1}, q_{j, k, l}^{B, i+1}, q_{j, k, l}^{D, i+1}$ y $q_{j, k, l}^{U, i+1}$ :

$$
\begin{aligned}
& q_{j, k, l}^{L, i+1}= \begin{cases}\frac{2 K_{j, k, l}^{L, i}}{\Delta x_{j}}\left[h_{j, k, l}^{i+1}+\frac{1}{2}\left(z_{l}+z_{l+1}\right)-\lambda_{j, k, l}^{1, i+1}\right] & j=2, \ldots, N_{x} \\
0 & j=1,\end{cases} \\
& q_{j, k, l}^{R, i+1}= \begin{cases}\frac{2 K_{j, k, l}^{R, i}}{\Delta x_{j}}\left[h_{j, k, l}^{i+1}+\frac{1}{2}\left(z_{l}+z_{l+1}\right)-\lambda_{j+1, k, l}^{1, i+1}\right] & j=1, \ldots, N_{x}-1 \\
0 & j=N_{x},\end{cases} \\
& q_{j, k, l}^{F, i+1}= \begin{cases}\frac{2 K_{j, k, l}^{F, i}}{\Delta y_{k}}\left[h_{j, k, l}^{i+1}+\frac{1}{2}\left(z_{l}+z_{l+1}\right)-\lambda_{j, k, l}^{2, i+1}\right] & k=2, \ldots, N_{y} \\
0 & k=1,\end{cases} \\
& q_{j, k, l}^{B, i+1}= \begin{cases}\frac{2 K_{j, k, l}^{B, i}}{\Delta y_{k}}\left[h_{j, k, l}^{i+1}+\frac{1}{2}\left(z_{l}+z_{l+1}\right)-\lambda_{j, k+1, l}^{2, i+1}\right] & k=1, \ldots, N_{y}-1 \\
0 & k=N_{y},\end{cases} \\
& q_{j, k, l}^{D, i+1}= \begin{cases}\frac{2 K_{j, k, l}^{D, i}}{\Delta z_{l}}\left[h_{j, k, l}^{i+1}+\frac{1}{2}\left(z_{l}+z_{l+1}\right)-\lambda_{j, k, l}^{3, i+1}\right] & l=2, \ldots, N_{z} \\
\frac{2 K_{j, k}^{D, i},}{\Delta z_{l}}\left[h_{j, k, l}^{i+1}+\frac{1}{2}\left(z_{l}+z_{l+1}\right)-h^{D}-z_{l}\right] & l=1,\end{cases} \\
& q_{j, k, l}^{U, i+1}= \begin{cases}\frac{2 K_{j, k, l}^{U, i}}{\Delta z_{l}}\left[h_{j, k, l}^{i+1}+\frac{1}{2}\left(z_{l}+z_{l+1}\right)-\lambda_{j, k, l+1}^{3, i+1}\right] & l=1, \ldots, N_{z}-1 \\
\frac{2 K_{j, k, l}^{U, i}}{\Delta z_{l}}\left[h_{j, k, l}^{i+1}+\frac{1}{2}\left(z_{l}+z_{l+1}\right)-h^{U}-z_{l+1}\right] & l=N_{z},\end{cases}
\end{aligned}
$$


siendo

$$
\begin{array}{ll}
K_{j, k, l}^{L, i}=K\left(\lambda_{j, k, l}^{1, i}-\frac{\left(z_{l}+z_{l+1}\right)}{2}\right), & K_{j, k, l}^{R, i}=K\left(\lambda_{j+1, k, l}^{1, i}-\frac{\left(z_{l}+z_{l+1}\right)}{2}\right), \\
K_{j, k, l}^{F, i}=K\left(\lambda_{j, k, l}^{2, i}-\frac{\left(z_{l}+z_{l+1}\right)}{2}\right), & K_{j, k, l}^{B, i}=K\left(\lambda_{j, k+1, l}^{2, i}-\frac{\left(z_{l}+z_{l+1}\right)}{2}\right), \\
K_{j, k, l}^{D, i}=K\left(\lambda_{j, k, l}^{3, i}-z_{l}\right), & K_{j, k, l}^{U, i}=K\left(\lambda_{j, k, l+1}^{3, i}-z_{l+1}\right) .
\end{array}
$$

Notar que para evaluar $K(h)$ en los bordes se han utilizado los multiplicadores de Lagrange.

La ecuación (A.23) equivale a:

$$
\begin{array}{rl}
q_{j, k, l}^{L, i+1}+q_{j-1, k, l}^{R, i+1}=0 & j=2, \ldots, N_{x}, \quad k=1, \ldots, N_{y}, \quad l=1, \ldots, N_{z}, \\
q_{j, k, l}^{F, i+1}+q_{j, k-1, l}^{B, i+1}=0 & j=1, \ldots, N_{x}, \quad k=2, \ldots, N_{y}, \quad l=1, \ldots, N_{z}, \\
q_{j, k, l}^{D, i+1}+q_{j, k, l-1}^{U, i+1}=0 & j=1, \ldots, N_{x}, \quad k=1, \ldots, N_{y}, \quad l=2, \ldots, N_{z} .
\end{array}
$$

Reemplazando (A.35)-(A.40) en (A.42)-(A.44) se obtienen las siguientes expresiones para los multiplicadores de Lagrange $\lambda_{j, k, l}^{s, i+1}$ en términos de la altura de presión $h_{j, k, l}^{i+1}$ :

$$
\begin{aligned}
\lambda_{j, k, l}^{1, i+1}= & \frac{K_{j, k, l}^{L, i} \Delta x_{j-1}}{\Delta x_{j-1} K_{j, i, l}^{L, i}+\Delta x_{j} K_{j-1, k, l}^{R, i}} h_{j, k, l}^{i+1} \\
+ & \frac{K_{j, 1, k, l}^{R, i} h x_{j}}{\Delta x_{j-1} K_{j, k, l}^{L, i}+\Delta x_{j} K_{j-1, k, l}^{R, i}} h_{j-1, k, l}^{i+1}+\frac{1}{2}\left(z_{k+1}+z_{k}\right), \\
& j=2, \ldots, N_{x}, \quad k=1, \ldots, N_{y}, \quad l=1, \ldots, N_{z}, \\
\lambda_{j, k, l}^{2, i+1}= & \frac{K_{j, k, l}^{F, i} \Delta y_{k-1}}{\Delta y_{k-1} K_{j, k, l}^{F, i}+\Delta y_{k} K_{j, k-1, l}^{B, i}} h_{j, k, l}^{i+1} \\
+ & \frac{K_{j, k-1, l}^{B, i} h y_{k}}{h y_{k-1} K_{j, k, l}^{F, i}+\Delta y_{k} K_{j, k-1, l}^{B, i}} h_{j, k-1, l}^{i+1}+\frac{1}{2}\left(z_{k+1}+z_{k}\right), \\
& j=1, \ldots, N_{x}, \quad k=2, \ldots, N_{y}, \quad l=1, \ldots, N_{z}, \\
\lambda_{j, k, l}^{3, i+1}= & \frac{K_{j, k, l}^{D, i} \Delta z_{l-1}}{\Delta z_{l-1} K_{j, k, l}^{D, i}+\Delta z_{l} K_{j, k, l-1}^{U, i}}\left[h_{j, k, l}^{i+1}+\frac{1}{2}\left(z_{l}+z_{l+1}\right)\right] \\
+ & \frac{K_{j, k, l-1}^{U, i} \Delta z_{l}}{\Delta z_{l-1} K_{j, k, l}^{D, i}+\Delta z_{l} K_{j, k, l-1}^{U, i}}\left[h_{j, k, l-1}^{i+1}+\frac{1}{2}\left(z_{l-1}+z_{l}\right)\right], \\
& j=1, \ldots, N_{x}, \quad k=1, \ldots, N_{y}, \quad l=2, \ldots, N_{z} .
\end{aligned}
$$


Tomando $\psi_{j, k, l}(x, y, z)$ en (A.21) se obtiene:

$$
\frac{q_{j, k, l}^{L, i+1}+q_{j, k, l}^{R, i+1}}{\Delta x_{j}}+\frac{q_{j, k, l}^{F, i+1}+q_{j, k, l}^{B, i+1}}{\Delta y_{k}}+\frac{q_{j, k, l}^{D, i+1}+q_{j, k, l}^{U, i+1}}{\Delta z_{l}}=0 .
$$

Finalmente, reemplazando (A.35)-(A.40) y (A.45)-(A.47) en (A.48) se obtiene el siguiente sistema de ecuaciones:

$$
\begin{aligned}
& L_{j, k, l}^{i} h_{j-1, k, l}^{i+1}+F_{j, k, l}^{i} h_{j, k-1, l}^{i+1}+D_{j, k, l}^{i} h_{j, k, l-1}^{i+1}+C_{j, k, l}^{i} h_{j, k, l}^{i+1}+ \\
& R_{j, k, l}^{i} h_{j+1, k, l}^{i+1}+B_{j, k, l}^{i} h_{j, k+1, l}^{i+1}+U_{j, k, l}^{i} h_{j, k, l+1}^{i+1}=R H S_{j, k, l}^{i} .
\end{aligned}
$$

siendo

$$
\begin{gathered}
L_{j, k, l}^{i}=-\left[1-\delta_{1, j}\right] \frac{A_{j, k, l}^{L, i},}{\Delta x_{j}}, \quad R_{j, k, l}^{i}=-\left[1-\delta_{N_{x}, j}\right] \frac{A_{j, k, l}^{R, i}}{\Delta x_{j}} \\
F_{j, k, l}^{i}=-\left[1-\delta_{1, k}\right] \frac{A_{j, k, l}^{F, i}}{\Delta y_{k}}, \quad B_{j, k, l}^{i}=-\left[1-\delta_{N_{y}, k}\right] \frac{A_{j, k, l}^{B, i}}{\Delta y_{k}}, \\
D_{j, k, l}^{i}=-\left[1-\delta_{1, l}\right] \frac{A_{j, k, l}^{D, l}}{\Delta z_{l}}, \quad U_{j, k, l}^{i}=-\left[1-\delta_{N_{z}, l}\right] \frac{A_{j, k, l}^{U, i}}{\Delta z_{l}}, \\
C_{j, k, l}^{i}=\left[1-\delta_{1, j}\right] \frac{A_{j, k, l}^{L,,}}{\Delta x_{j}}+\left[1-\delta_{1, k}\right] \frac{A_{j, k, l}^{F, i}}{\Delta y_{k}}+\left[1-\delta_{1, l}\right] \frac{A_{j, k, l}^{D, i}}{\Delta z_{l}} \\
+\left[1-\delta_{N_{x}, j}\right] \frac{A_{j, k, l}^{R, i}}{\Delta x_{j}}+\left[1-\delta_{N_{y}, k}\right] \frac{A_{j, k, l}^{B, i}}{\Delta y_{k}}+\left[1-\delta_{N_{z}, l}\right] \frac{A_{j, k, l}^{U, i}}{\Delta z_{l}} \\
+\frac{2 K_{j, k, l}^{D D,}}{\Delta z_{l}^{2}} \delta_{1, l}+\frac{2 K_{j, k, l}^{U, i}}{\Delta z_{l}^{2}} \delta_{N_{z}, l} \\
R H S_{j, k, l}^{i}=\delta_{1, l} \frac{2 K_{j, k, l}^{D, i}\left(h^{D}-\Delta z_{l}\right)}{\Delta z_{l}^{2}}+\delta_{N_{z}, l} \frac{2 K_{j, k, l}^{U, i}\left(h^{U}+\Delta z_{l}\right)}{\Delta z_{l}^{2}} \\
-\left[1-\delta_{1, l}\right] \frac{A_{j, k, l}^{D, i}}{\Delta z_{l}} \frac{\left(z_{l+1}-z_{l-1}\right)}{2}+\left[1-\delta_{N_{z}, l}\right] \frac{A_{j, k, l}^{U, i}}{\Delta z_{l}} \frac{\left(z_{l+2}-z_{l}\right)}{2}
\end{gathered}
$$

donde:

$$
\begin{aligned}
A_{j, k, l}^{L, i} & =\frac{2 K_{j, k, l}^{L, i} K_{j-1, k, l}^{R, i}}{\Delta x_{j-1} K_{j, k, l}^{L, i}+\Delta x_{j} K_{j-1, k, l}^{R, i}}, & A_{j, k, l}^{R, i} & =\frac{2 K_{j+1, k, l}^{L, i} K_{j, k, l}^{R, i}}{\Delta x_{j} K_{j+1, k, l}^{L, i}+\Delta x_{j+1} K_{j, k, l}^{R, i},}, \\
A_{j, k, l}^{F, i} & =\frac{2 K_{j, k, l}^{F, i} K_{j, k-1, l}^{B, i}}{\Delta y_{k-1} K_{j, k, l}^{F, i}+\Delta y_{k} K_{j, k-1, l}^{B, i}}, & A_{j, k, l}^{B, i} & =\frac{2 K_{j, k+1, l}^{F, i} K_{j, k, l}^{B, i}}{\Delta y_{k} K_{j, k+1, l}^{L, i}+\Delta y_{k+1} K_{j, k, l}^{B, i}}, \\
A_{j, k, l}^{D, i} & =\frac{2 K_{j, k, l}^{D, i} K_{j, k, l-1}^{U, i}}{\Delta z_{l-1} K_{j, k, l}^{D, i}+\Delta z_{l} K_{j, k, l-1}^{U, i}}, & A_{j, k, l}^{U, i} & =\frac{2 K_{j, k, l+1}^{D, i} K_{j, k, l}^{U, i}}{\Delta z_{l} K_{j, k, l+1}^{D, i}+\Delta z_{l+1} K_{j, k, l}^{U, i}},
\end{aligned}
$$


y $\delta_{j, k}$ es la Delta de Kronecker:

$$
\delta_{j, k}= \begin{cases}1, & j=k \\ 0, & j \neq k .\end{cases}
$$

\section{A.5 Esquema general de la simulación}

El código computacional para resolver el sistema de ecuaciones resultante fue desarrollado en lenguaje Fortran. La estructura del algoritmo puede resumirse en los siguientes pasos:

1. Se asigna un valor inicial para el proceso iterativo de Picard considerando la siguiente expresión para $h^{0}(x, y, z)$ :

$$
h^{0}(x, y, z)=\left(\frac{h^{U}-h^{D}}{L_{z}}\right) z+h^{D} .
$$

2. Se resuelve el sistema (A.49) y se obtiene $h_{j, k, l}^{i+1}$.

3. Se evalúa el módulo de la diferencia $\delta h_{j, k, l}^{i+1}=h_{j, k, l}^{i+1}-h_{j, k, l}^{i}$ sobre todo el dominio. Si la norma de $\delta h_{j, k, l}^{i+1}$ es menor que cierta tolerancia prefijada, la iteración concluye y $h_{j, k, l}^{i+1}$ es la solución numérica deseada. De lo contrario, se vuelve al paso 2 definiendo $h_{j, k, l}^{i}=h_{j, k, l}^{i+1}$.

4. Se calculan los valores del campo de flujo $q_{j, k, l}^{S, i+1}(S=L, R, F, B, D, U)$ utilizando (A.35)-(A.40).

El sistema de ecuaciones del paso 2 se resuelve utilizando un método de sobrerrelajación por líneas (Método LSOR [79]). 


\section{Referencias}

[1] Abramowitz, M. and I.A. Stegun. Handbook of mathematical functions with formulas, graphs, and mathematical tables. Dover, New York, 1st edition, 1965.

[2] Acuna, J.A. and Y.C. Yortsos. Application of fractal geometry to the study of networks of fractures and their pressure transient. Water Resources Research, 31:527$540,1995$.

[3] Arfken, G.B. and H.J. Weber. Mathematical methods for physicists. Harcourt, San Diego, 6th edition, 2005.

[4] Arnold, D.N. and F. Brezzi. Mixed and nonconforming finite element methods: implementation, postprocessing and error estimates. R.A.I.R.O. Modélisation Mathématique et Analyse Numérique, 19:7-32, 1985.

[5] Bachu, S. $\mathrm{CO}_{2}$ storage in geological media: Role, means, status and barriers to deployment. Progress in Energy and Combustion Science, 34:254-273, 2008.

[6] Barton, C.A. and M.D. Zoback. Self-similar distribution and properties of macroscopic fractures at depth in cristalline rock in the Cajon Pass Scientific Drill Hole. Journal of Geophysical Research, 97:5181-5200, 1992.

[7] Bear, J. Dynamics of fluids in porous media. Dover, New York, 1st edition, 1988.

[8] Berkowitz, B. Characterizing flow and transport in fractured geological media: a review. Advances in Water Resources, 25:861-884, 2002.

[9] Berkowitz, B. and A. Hadad. Fractal and multifractal measure of natural and synthetic fracture networks. Journal of Geophysical Research, 102:205-218, 1997.

[10] Bodvarsson, G.S. and Y. Tsang. Yucca Mountain project. Journal of Contaminant Hydrology, 38:1-146, 1999.

[11] Bonnet, E, O. Bour, N. Odling, P. Davy, I. Main, P. Cowie, and B. Berkowitz. Scaling of Fracture Systems in Geological Media. Reviews of Geophysics, 39:347383, 2001. 
Referencias

[12] Bossart, P. and M. Mazurek. Grimsel Test Site: Structural Geology and Water Flow-Paths in the Migration Shear-Zone. Nagra Technical Report 92-12, Nagra, Wettingen, Switzerland, 1991.

[13] Brooks, R.H. and A.T. Corey. Hydraulic Properties of Porous Media. Hydrol. Pap. No. 3. Colorado State University, Fort Collins, 1964.

[14] Buckingham, E. Studies on the movement of soil moisture. Bureau of Soil Bulletin, 38:29-61, 1907.

[15] Burdine, N.T. Relative permeability from pore size distribution data. Trans. Am. Inst. Min. Metall. Pet. Eng., 198:71-78, 1953.

[16] Cardenas, M.B. Lessons from and assessment of Boussinesq aquifer modeling of a large fluvial island in a dam-regulated river. Advances in Water Resources, 33(11):1359-1366, 2010.

[17] Carol, E.S., Kruse E.E., Pousa J.L., and Roig A.R. Determination of heterogeneities in the hydraulic properties of a phreatic aquifer from tidal level fluctuations: a case in Argentina. Hydrogeology Journal, 17(7):1727-1732, 2009.

[18] Carr, P.A. and G. van der Kamp. Determining aquifer characteristics by the tidal methods. Water Resources Research, 5(5):1023-1031, 1969.

[19] Carsel, R.S. and R.S. Parrish. Developing join probability distributions of soil water characteristics. Water Resources Research, 24:755-769, 1998.

[20] Celia, M.A. and P. Binning. A mass conservative numerical solution for two-phase flow in porous media with application to unsaturated flow. Water Resources Research, 28:2819-2828, 1992.

[21] Celia, M.A., E.T. Bouloutas, and R.L. Zarba. A general mass-conservative numerical solution for the unsaturated flow equation. Water Resources Research, 26:1483-1496, 1990.

[22] Cesanelli, A. and L. Guarracino. Estimation of actual evapotranspiration by numerical modeling of water flow in the unsaturated zone: a case study in Buenos Aires, Argentina. Hydrogeology Journal, 17:299-306, 2009.

[23] Chuang, M.H., C.S. Huang, G.H. Li, and H.D. Yeh. Groundwater fluctuations in heterogeneous coastal leaky aquifer systems. Hydrology and Earth System Sciences, 14:1819-1826, 2010. 
[24] Dagan, G. Flow and transport in Porous Formations, volume I. Springer-Verlag, 1989.

[25] Desbarats, A.J. Spatial averaging of hydraulic conductivity in three-dimensional heterogeneous porous media. Mathematical Geology, 24:249-267, 1992.

[26] Doughty, C. and K. Karasaki. Flow and transport in hierarchically fractured rock. Journal of Hydrology, 263:1-22, 2002.

[27] Drogue, C., M. Rezark, and A. Krivic. Survey of a coastal karstic aquifer by analysis of the effect of Kras of Slovenia, Yugoslavia. Environmental Geology and Water Sciences, 6:103-109, 1984.

[28] Dury, O., U. Fischer, and R. Schulin. A comparison of relative nonwetting-phase permeability models. Water Resources Research, 35:1481-1493, 1999.

[29] Erskine, A.D. The effect of tidal fluctuation on a coastal aquifer in the UK. Ground Water, 29:556-562, 1991.

[30] Ferris, J.G. Cyclic fluctuations of water level as a basis for determining aquifer transmissibility. Int. Assoc. Sci. Hydrol. Pub., 33:148-155, 1951.

[31] Fetter, C.W. Contaminant Hydrogeology. Prentice-Hall, New Jersey, 1992.

[32] Fischer, U., B. Kulli, and H. Flüler. Constitutive relationships and pore structure of undisturbed fracture zone samples with cohesionless fault gouge layers. Water Resources Research, 34:1695-1701, 1998.

[33] Flint, A.L., E.L. Flint, G. S. Bodvarsson, E. M. Kwicklis, and J. Fabryka-Martin. Evolution of the conceptual model of unsaturated zone hydrology al Yucca Mountain, Nevada. Journal of Hydrology, 247:1-30, 2001.

[34] Freeze, R.A. and J.A. Cherry. Groundwater. Prentice-Hall, Englewood Cliffs, NJ, 1979.

[35] Gardner, W.R. Some steady state solutions of unsaturated moisture flow equations with application to evaporation from a water table. Soil Sci., 85:228-232, 1958.

[36] Geng, X., H. Li, and M.C. Boufadel. Tide-induced head fluctuations in a coastal aquifer: effects of the elastic storage and leakage of the submarine outlet-capping. Hydrogeology Journal, 17:1289-1296, 2009. 
[37] Gómez, A.A., L.B. Rodriguez, and L.S. Vives. Finite difference model for evaluating the recharge of the Guaraní Aquifer System on the Uruguayan Brazilian border. Mecánica Computacional XXV, pages 1479-1496, 2006.

[38] Guarracino, L. Estimation of saturated hydraulic conductivity $K_{s}$ from the van Genuchten shape parameter $\alpha$. Water Resources Research, 43, 2004.

[39] Guarracino, L. A fractal constitutive model for unsaturated flow in fracured hard rocks. Journal of Hydrology, 324:154-162, 2006.

[40] Guarracino, L., J. Carrera, and E. Vázquez-Suñé. Analytical study of hydraulic and mechanical effects on tide-induced head fluctuation in a coastal aquifer system under the sea. Journal of Hydrology, 450-451:150-158, 2012.

[41] Guarracino, L. and L. Monachesi. Numerical simulation of constitutive relations for unsaturated flow in fractured porous media. XVIII International Conference on Computational Methods in Water Resources, pages 1-8, 2010.

[42] Guarracino, L. and F. Quintana. A constitutive model for flow in unsaturated fractured rocks. Hydrological Processes, 23:697-701, 2009.

[43] Guo, H., J.J. Jiao, and H. Li. Groundwater response to tidal fluctuations in a two-zone aquifer. Journal of Hydrology, 381:364-371, 2010.

[44] Jacob, C.E. Flow of groundwater. In: Rouse $H$ (ed) Engineering hydraulics. Wiley, New York, 1950.

[45] Jeng, D.S., L. Li, and D.A. Barry. Analytical solution for tidal propagation in a coupled semi-confined/phreatic coastal aquifer. Advances in Water Resources, 25:577-584, 2002.

[46] Jha, M.K., Y. Kamii, and K. Chikamori. On the estimation of phreatic aquifer parameters by the tidal response technique. Water Resources Management, 17:69$88,2003$.

[47] Jiao, J.J. and Z. Tang. An analytical solution of groundwater response to tidal fluctuation in a leaky confined aquifer. Water Resources Research, 35:747-775, 1999.

[48] Journel, A.G. and Ch.J. Huijbregts. Mining geostatistics. Academic Press, London, 1978. 
[49] Klute, A. Laboratory measurements of hydraulic conductivity of unsaturated soil, in Methods of soil analysis. C. A. Black Ed., 1965.

[50] Knudsen, M. The kinetic theory of gases. Methuen, London, 1934.

[51] Kwicklis, E.M. and R.W. Healey. Numerical investigation of steady liquid water flow in a variably saturated fractured network. Water Resources Research, 29:40914102, 1993.

[52] Li, G., H. Li, and Boufadel M.C. The enhancing effect of the elastic storage of the seabed aquitard on the tide-induced groundwater head fluctuation in confined submarine aquifer systems. Journal of Hydrology, 350:83-92, 2008.

[53] Li, H. and J.J. Jiao. Analytical studies of groundwater head fluctuation in a coastal confined aquifer overlain by a semi-permeable layer with storage. Advances in Water Resources, 24:565-573, 2001.

[54] Li, H. and J.J. Jiao. Influence of tide on the mean watertable in an unconfined, anisotropic, inhomogeneous coastal aquifer. Advances in Water Resources, 26:9-16, 2003.

[55] Li, H.L. and J.J. Jiao. Analytical solutions of tidal groundwater flow in coastal two-aquifer system. Advances in Water Resources, 25:417-426, 2002.

[56] Li, H.L. and J.J. Jiao. Tidal groundwater level fluctuations in L-shaped leaky coastal aquifer system. Journal of Hydrology, 268:234-243, 2002.

[57] Li, H.L. and J.J. Jiao. Tide-induced seawater-groundwater circulation in a multilayered coastal leaky aquifer system. Journal of Hydrology, 274:211-224, 2003.

[58] Li, H.L., J.J. Jiao, M. Luk, and K. Cheung. Tide-induced groundwater level fluctuation in coastal aquifers bounded by L-shaped coastlines. Water Resources Research, 38(3):6-1-6-8, 2002.

[59] Liu, H.H. and G.S. Bodvarsson. Constitutive relations for unsaturated flow in fracture networks. Journal of Hydrology, 252:116-125, 2001.

[60] Lunt, I.A., D.I. Siegel, and R.I. Bauer. A quantitative, three-dimensional depositional model of gravity braided rivers. Sedimentology, 51:377-414, 2004.

[61] Mandelbrot, B.B. The fractal geometry of nature. W. H. Freeman, New York, 1983. 
[62] Mantoglou, A. and J.L. Wilson. The turning bands method for simulation of random fields using line generation by a spectral method. Water Resources Research, 18:1379-1394, 1982.

[63] Marsily, G. Quantitative Hidrogeology. Academic Press INC, Florida, 1st edition, 1986.

[64] Millham, N.P. and B.L. Howes. A comparison of methods to determine $K$ in a shallow coastal aquifer. Ground Water, 33:49-57, 1995.

[65] Monachesi, L.B. and L. Guarracino. Simulación numérica de relaciones constitutivas para el flujo no saturado en rocas fracturadas. Actas del I Congreso Internacional de Hidrología de Llanuras, 1:295-302, 2010.

[66] Monachesi, L.B. and L. Guarracino. Un modelo fractal para estimar la conductividad hidráulica no saturada de rocas fracturadas. GEOACTA, 35:66-77, 2010.

[67] Monachesi, L.B. and L. Guarracino. Estimación de la conductividad hidráulca en acuíferos costeros heterogéneos a partir de las fluctuaciones de altura piezométrica inducidas por marea. Actas del VII Congreso Argentino de Hidrologeología, 1:67, 2011.

[68] Monachesi, L.B. and L. Guarracino. Exact and approximate analytical solutions of groundwater response to tidal fluctuations in a theoretical inhomogeneous coastal confined aquifer. Hydrogeology Journal, 19:1443-1449, 2011.

[69] Monachesi, L.B. and L. Guarracino. A fractal model for predicting water and air permeabilities of unsaturated fractured rocks. Transport in Porous Media, 90:779789, 2011.

[70] Monachesi, L.B. and L. Guarracino. Un método numérico para la obtención de relaciones constitutivas en rocas fracturadas. Cuadernos del CURIHAM, 17:1-8, 2011.

[71] Monachesi, L.B. and L. Guarracino. Conductividad hidráulica efectiva de medios porosos heterogéneos 2D y 3D. Actas del X Congreso Argentino de Mecánica Computacional, 31:509-518, 2012. 
[72] Montalto, F.A., T.S. Steenhuis, and J.Y. Parlange. The hydrology of Piermont Marsh, a reference for tidal marsh restoration in the Hudson River estuary, New York. Journal of Hydrology, 316:108-128, 2006.

[73] Mualem, Y. A new model for predicting the hydraulic conductivity of unsaturated porous media. Water Resources Research, 12:513-522, 1976.

[74] Müller, T., Lambert G., and B. Gurevich. Dynamic permeability of porous rocks and its seismic signatures. Geophysics, 72:E149-E158, 2007.

[75] Müller, T., B. Gurevich, and M. Lebedev. Seismic wave attenuation and dispersion resulting from wave induced flow in porous rocks-A review. Geophysics, 75:75A147-75A164, 2010.

[76] Nedelec, J. Mixed finite elements in $R^{3}$. Numer. Math., 35:315-341, 1980.

[77] Obuko, P.G. and K. Aki. Fractal geometry in the San Andreas fault system. Journal of Geophysical Research, 92:345-355, 1987.

[78] Or, D. and M. Tuller. Hydraulic conductivity of partially saturated fractured porous media: flow in a cross section. Advances in Water Resources, 26:883-898, 2003.

[79] Peaceman, D.W. Fundamentals of numerical reservoir simulation. Elsevier, 1977.

[80] Pendleton, D.E., A. Dathe, and P. Baveye. Influence of image resolution and evaluation algorithm on estimates of the lacunarity of porous media. Physical Review E, 72:9pp, 2005.

[81] Prandtl, L. and O.G. Teitjens. Fundamentals of hydro-and aeromechanics. Mc. Graw Hill, New York, 1934.

[82] Pruess, K. and Y.W. Tsang. On two-phase relative permeability and capillary pressure of rough-walled rock fractures. Water Resources Research, 26:1915-1926, 1990.

[83] Raviart, P.A. and J.M. Thomas. A mixed finite element method for second order elliptic problems: Mathematical aspects of the finite element method. Lect. Notes Math., 606:292-315, 1977.

[84] Richards, L. Capillary conduction of liquids through porous mediums. Physics, 1:318-333, 1931. 
[85] Rosa Filho, E.F., E.C. Hindi, and D.A. Giusti. Sistema Aquífero Guarani: consideraçoes preliminares sobre a influencia de Arco de Ponta Grossa no Fluxo das águas subterraneas. Águas Subterr, 17:91-111, 2003.

[86] Rubino, G., L. Monachesi, L. Guarracino, T. Müller, and K. Holliger. Do seismic waves and fluid flow sense the same permeability? Actas del 74th EAGE Conference and Exhibition incorporating SPE EUROPEC, 2012.

[87] Rubino, G., L. Monachesi, L. Guarracino, T. Müller, and K. Holliger. Dynamic permeability of heterogeneous porous rocks having strong permeability fluctuations and its effects on seismic attenuation and velocity dispersion. Actas de la SEG International Exposition and Eighty-Second Annual Meeting, 2012.

[88] Rubino, J.G. Atenuación y dispersión de ondas sísmicas en medios porosos saturados altamente heterogéneos. PhD thesis, Universidad Nacional de La Plata, 2008.

[89] Russo, D. Determining soil hydraulic properties by parameter estimation : On the selection of a model for the hydraulic properties. Water Resources Research, 24:453-459, 1988.

[90] Russo, D. and M. Bouton. Statistical analysis of spatial variability in unsaturated flow parameters. Water Resources Research, 28:1911-1925, 1992.

[91] Russo, D., I. Russo, and A. Laufer. On the spatial variability of parameters of the unsaturated hydraulic conductivity. Water Resources Research, 33:945-956, 1997.

[92] Sanchez-Vila, X., J. P. Girardi, and J. Carrera. A synthesis of approaches to upscaling of hydraulic conductivities. Water Resources Research, 31:867-882, 1995.

[93] Schultz, G. and C. Ruppel. Constraints on hydraulic parameters and implications for groundwater flux across the upland-estuary interface. Journal of Hydrology, 260:255-269, 2002.

[94] Sen, M. and P.L. Stoffa. Global Optimization Methods in Geophysical Inversion. Elsevier, Amsterdam, 1995.

[95] Serfes, M.E. Determining the mean hydraulic gradient of ground water affected by tidal fluctuations. Ground Water, 29:549-555, 1991. 
[96] Sleep, B.E. and J.F. Sykes. Modeling the transport of volatile organics in variably saturated media. Water Resources Research, 25:81-82, 1989.

[97] Song, Z., L. Li, J. Kong, and H. Zhang. A new analytical solution of tidal water table fluctuations in a coastal unconfined aquifer. Journal of Hydrology, 340:256$260,2007$.

[98] Stonestrom, D.A. and J. Rubin. Air permeability and trapped air content in two soils. Water Resources Research, 25:1959-1969, 1989.

[99] Sun, P., H.L. Li, M.C. Boufadel, X.L. Geng, and S. Chen. An analytical solution and case study of groundwater head response to dual tide in an island leaky confined aquifer. Water Resources Research, 44:7pp, 2008.

[100] Trefry, M.G. Periodic forcing in composite aquifers. Advances in Water Resources, 22:645-656, 1999.

[101] Trefry, M.G. and E. Bekele. Structural characterization of an island aquifer via tidal methods. Water Resources Research, 40:21pp, 2004.

[102] Trefry, M.G. and C.D. Johnston. Pumping test analysis for a tidally forced aquifer. Ground Water, 36:427-433, 1998.

[103] Tuller, M. and D. Or. Unsaturated hydraulic conductivity of structured porous media: a review of liquid configuration-based models. Vadose Zone Journal, 1:1437, 2002.

[104] Turcotte, D.L. Fractals and fragmentation. Journal of Geophysical Research, 91:1921-1926, 1986.

[105] Turcotte, D.L. Fractals and Chaos in Geology and Geophysics fragmentation. Cambridge University Press, New York, 1997.

[106] Tyler, S.W. and S.W. Wheatcraft. Fractal process in soil water retention. Water Resources Research, 26:1047-1054, 1990.

[107] Unger, J.A., B. Faybishenko, G.S. Bodvarsson, and A.M. Simmons. Simulating infiltration tests in fractured basalt at the Box Canyon Site, Idaho. Vadose Zone Journal, 3:75-89, 2004.

[108] van Genuchten, M. A closed form equation for predicting the hydraulic conductivity of unsaturated soils. Soil Sci. Soc. Am. J., 40:892-898, 1980. 
[109] Villholth, K.G, K.H. Jensen, and J. Fredericia. Flow and transport processes in a macroporous subsurface-drained glacial till soil. Journal of Hydrology, 207:98-120, 1998.

[110] Wierenga, P.J., R.G. Hills, and D.B. Hudson. The Las Cruces trench site: Characterization, experimental results, and one-dimensional flow predictions. Water Resources Research, 27:2695-2705, 1991.

[111] Wyllie, M.R.J. and G.H.F. Gardner. The generalized Kozeny-Carman equation II. A novel approach to problems of fluid flow. World Oil Prod. Sect., pages 210-228, 1958.

[112] Yu, B. Analysis of flow in fractal porous media. Applied Mechanics Reviews, 61:19pp, 2008.

[113] Zhang, S. and Jin, J.M. Computation of special functions. A Wiley-Interscience Publication. John Wiley, 1996.

[114] Zoback, M.D. Reservoir Geomechanics. Cambridge University Press, New York, 2007. 